\title{
Exploring the genetics underlying the Zn deficiency response in Arabidopsis thaliana
}




\section{Thesis committee}

\section{Promotor}

Prof. Dr M.G.M. Aarts

Personal chair at the Laboratory of Genetics

Wageningen University \& Research

\section{Co-promotor}

Prof. Dr M. Koornneef

Personal chair at the Laboratory of Genetics

Wageningen University \& Research

\section{Other members}

Prof. Dr G.H. Immink, Wageningen University \& Research

Dr H. Schat, VU Amsterdam

Dr A.G.L. Assunção, University of Copenhagen, Denmark

Dr S. Merlot, French National Centre for Scientific Research (CNRS), Gif sur Yvette, France

This research was conducted under the auspices of the Graduate School of Experimental Plant Sciences (EPS). 


\title{
Exploring the genetics underlying the Zn deficiency response in Arabidopsis thaliana
}

\author{
Andrea Valeria Ochoa Tufiño
}

\section{Thesis}

submitted in fulfilment of the requirements for the degree of doctor

at Wageningen University

by the authority of the Rector Magnificus,

Prof. Dr A.P.J. Mol,

in the presence of the

Thesis Committee appointed by the Academic Board

to be defended in public

on Friday 5 October 2018

at 11 a.m. in the Aula. 
Andrea Valeria Ochoa Tufiño

Exploring the genetics underlying the Zn deficiency response in Arabidopsis thaliana, 218 pages.

PhD thesis, Wageningen University, Wageningen, the Netherlands (2018)

With references, with summary in English

ISBN 978-94-6343-339-6

DOI https://doi.org/10.18174/458236 


\section{Table of Contents}

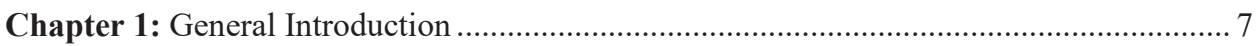

Chapter 2: Uncovering Zn transporters induced by Zn deficiency.................................. 17

Chapter 3: Disruption of N-terminal-acetylation disturbs mineral homeostasis in Arabidopsis thaliana

Chapter 4: Exploring Arabidopsis thaliana natural genetic variation to identify genes underlying the $\mathrm{Zn}$ deficiency response

Chapter 5: A conserver cluster of tandemly arrayed HIPP genes affects tolerance to Fe and $\mathrm{Zn}$ deficiency in Arabidopsis thaliana 123

Chapter 6: General Discussion 155

References 171

Summary 207

Acknowledgments 211

Curriculum Vitae 213

Educational Statement. 215 



\section{Chapter 1}

General Introduction 


\section{Zinc is essential for life}

Zinc $(\mathrm{Zn})$ is an essential element for all living organisms. It is involved in a wide range of biological processes due to its particular chemistry, which makes biological systems choose $\mathrm{Zn}$, rather than other elements, such as Iron $(\mathrm{Fe})$ or Copper $(\mathrm{Cu})$ in certain biochemical processes. Firstly, the oxidation state $(+2)$ of $\mathrm{Zn}$ does not change under physiological conditions, due to the lack of unpaired electrons and its electron configuration with no unfilled d subshells (Barak and Helm ke, 1993; Krężel and Maret, 2016) Therefore, I will refer to $\mathrm{Zn}^{2+}$ simply as $\mathrm{Zn}$ in the rest of this thesis. The $\mathrm{Zn}$ redox stability favours the role of $\mathrm{Zn}$ as the cofactor of enzymes involved in redox reactions. The kind of reactions mentioned above can lead to the generation of damaging radicals in delicate processes, such as DNA or RNA synthesis (Park et al., 1999). Secondly, Zn has low coordination number sites that are strongly acidic. The $\mathrm{Zn}$ coordination is specific for Sulphur (S), Nitrogen $(\mathrm{N})$, and Oxygen (O). Consequently, it binds to the side chains of histidine, glutamate, aspartate, and/or cysteine in proteins (Barak and Helmke, 1993). Thirdly, Zn has flexible coordination geometries with different ligands, this feature facilitates the reactions of enzymes of catalysis (Williams, 1984).

Zinc was not considered as an essential element for any living organism until 1869, when J. Raulin, a student of Louis Pasteur, reported that $\mathrm{Zn}$ was required for growth of the fungus Aspergillus niger (Raulin, 1869). A half-century later, Mazé reported that Zea mays (maize) plants required $\mathrm{Zn}$ for growth (Mazé, 1914). However, this finding was questioned for inconsistent reproducibility. Fortunately, those results stimulated investigations to obtain more conclusive evidence. In 1926, Zn became generally recognised as essential for higher plants, after Sommer and Lipman showed the effects of $\mathrm{Zn}$ deficiency in Hordeum vulgare (barley) and Helianthus annuus (sunflower), which were grown hydroponically (Sommer and Lipman, 1926). In 1934, Zn was established as essential for higher animals by Todd, Elvehjem and Hart (Todd et al., 1980). In 1961, it was documented that $\mathrm{Zn}$ was essential for humans, after Prasad studied cases of Zn deficiency in Iran and Egypt (Prasad et al., 1963).

\section{Zinc deficiency}

An adult human body contains an average of 2.5 grams of $\mathrm{Zn}$, and a person should consume 8 to $15 \mathrm{mg}$ of $\mathrm{Zn}$ per day in the diet (Royal Society of Chemistry; U.S. Department of Health \& Human Services). Zinc deficiency affects two billion people in the world (Prasad, 2003; Müller and Krawinkel, 2005). Around 2800 human proteins contain zinc-binding domains, corresponding to $10 \%$ of the human proteome, $40 \%$ of them are transcription factors and the 
rest are mainly enzymes (Andreini et al., 2006). Zinc is involved in a multitude of biochemical processes associated with immunity, wound healing, growth, communication among cells, such as cells in the salivary gland or neurons (Roohani et al., 2013). Zn deficiency causes a broad range of symptoms, depending upon the severity of the condition. Mild $\mathrm{Zn}$ deficiency is the most important numerically (Roohani et al., 2013), and its symptoms are growth retardation, impaired immunity, cognitive impairment, neurological disorders, and delayed wound healing (Prasad, 2012). Zinc deficiency increases young children's risks to suffer diarrhoeal disease, pneumonia, and malaria (Caulfield and Black, 2004; Black et al., 2008), which accounts for $29 \%$ of all deaths of children under five years old (World Health Organisation; Global Health Observatory data).

One of the strategies to improve $\mathrm{Zn}$ status in vulnerable human populations is through biofortification of crops. This strategy aims to increase the nutrients content of staple food through agronomical practices and genetic improvement (Roohani et al., 2013). In low- and middleincome regions the population diet relies on staple food, such as cereals, if these crops are grown on $\mathrm{Zn}$ deficient soils, their edible parts will be $\mathrm{Zn}$ deficient, as well. Thus, in low- and middleincome regions, Zn deficiency in humans is strongly correlated with $\mathrm{Zn}$ deficiency in soils. (Müller and Krawinkel, 2005; Alloway, 2009; Beal et al., 2017).

After recognising $\mathrm{Zn}$ as an essential element for living organisms, people gave attention to $\mathrm{Zn}$ deficiency in the soil. In 1990, the FAO published a report on soil samples from around the world indicating that half of them were $\mathrm{Zn}$ deficient (FAO, Soils Bulletins). The availability of $\mathrm{Zn}$ to plants in soils is affected by factors, such as low $\mathrm{Zn}$ concentration, high $\mathrm{pH}$, high organic matter content, high moisture, and high concentrations of calcite, bicarbonate, Phosphorous (P), Magnesium ( $\mathrm{Mg}$ ), Sodium (Na), and Calcium (Ca). The low Zn concentration in soils can be solved by the application of fertilizers, as long as the deficiency is identified. When $\mathrm{Zn}$ deficiency is marginal in soils, symptoms of $\mathrm{Zn}$ deficiency in the crops are hidden and easily confused with deficiencies in other nutrients, such as Fe. As a result, the crops yield and quality are reduced without a clear reason (Alloway, 2009). In severe, Zn deficient soils, crops visible symptoms are: stunted growth, short internodes, interveinal chlorosis, small leaves, reddishbrown leaves, inward curled leaves and reduced yield (Cakmak, 2008; Alloway, 2009; Marschner and Marschner, 2012; Nielsen, 2012; Yruela, 2013; Mattiello et al., 2015). 


\section{Zn homeostasis in plants}

$\mathrm{Zn}$ homeostasis regulates the acquisition, redistribution and storing of $\mathrm{Zn}$ according to internal demands and external conditions (Sinclair and Krämer, 2012). Zn concentration varies in a narrow range within the cell, due to its buffering system, which is based on high-affinity binding to proteins and transport processes (Krężel and Maret, 2016). Zn homeostasis is driven by metal transporters, chelators, transcription factors, and post-transcription regulators (Sinclair and Krämer, 2012; Assunção et al., 2013).

$\mathrm{Zn}$ transport begins with the uptake of free $\mathrm{Zn}^{2+}$ from the soil across the plasma membrane of root cells. Within the symplast, Zn can be chelated (Haydon and Cobbett, 2007), or kept as free Zn (Sinclair and Krämer, 2012) to either be stored in root cell vacuoles or continue with radial transport until the stele (Claus et al., 2013). In the stele, $\mathrm{Zn}$ is uploaded into xylem to be translocated to the shoot. In shoot tissues, $\mathrm{Zn}$ is immediately used, stored or remobilised to different tissues via phloem to meet the plant requirements (Blindauer and Schmid, 2010; Sinclair and Krämer, 2012).

The $\mathrm{Zn}$ deficiency response cascade starts when plants are exposed to low $\mathrm{Zn}$ supply. This response is regulated by the transcription factors BASIC-REGION LEUCINE-ZIPPER 19 (bZIP19) and bZIP23. These two transcription factors work together to positively regulate the expression of genes mainly involved in Zn transport (Assunção et al., 2010), which facilitate the acquisition of $\mathrm{Zn}$ and its distribution in the plant.

The main transporters involved in the Zn root uptake belong to the ZRT-IRT-like protein (ZIP) family. The members of this ZIP family mainly transport Zn, however, they show variation in substrate range and specificity with other divalent cations, like Fe or Manganese (Mn). The coding sequences of ZIP1, ZIP2, ZIP3, ZIP4, ZIP5, ZIP6, IRT1, IRT2, or IRT3 are able to complement the yeast Zn-uptake-defective mutant (Korshunova et al., 1999; Vert et al., 2001; Lin et al., 2009; Assunção et al., 2010; Milner et al., 2013). ZIP proteins are associated with symplast Zn influx (Eide, 2006; Milner et al., 2013). Ten (ZIP1, ZIP3, ZIP4, ZIP5, ZIP9, ZIP10, ZIP11, ZIP12, and IRT3) of the fifteen members of the ZIP family, increase their expression under $\mathrm{Zn}$ deficiency with higher levels in roots than in shoots (Wintz et al., 2003; van de Mortel et al., 2006; Campos, 2015; Campos et al., 2017). 
For $\mathrm{Zn}$ to be translocated to shoots, it first needs to enter into the stele of the root. Roots are complex structures with different types of cells that contribute to nutrients acquisition, sequestration, and delivery to the xylem. Root nutrients, including $\mathrm{Zn}$, move radially from the outer root layers (epidermis, cortex, and endodermis) into the root vasculature for further translocation. Nutrients can move via the apoplastic route through cell walls and spaces between cells, via the symplastic route by transporters, which take them up into the cells and then move from cell to cell through plasmodesmata, or via a combination of both routes. The apoplastic route mainly takes place in younger zones of the root. As long as the root keeps getting older, it starts to develop the Casparian strip, which is a poorly ion-permeable belt that makes the endodermis a barrier to the free diffusion of solutes into the stele, and just allows the symplastic movement of nutrients (Clarkson, 1993; Tester and Leigh, 2001; Geldner, 2013; Barberon and Geldner, 2014). When the root becomes even older, a suberin layer develops around all sides of the endodermis, which just allows the cross of elements through plasmodesmata or passage cells to enter the root stele (Clarkson, 1993; Tester and Leigh, 2001; Geldner, 2013; Barberon and Geldner, 2014).

Within the root stele, the off-loading of $\mathrm{Zn}$ from parenchyma cells into the stele apoplast is facilitated by members of the Heavy-Metal-ATPases (HMA) family of cation transporters energised by hydrolysis of ATP. Within the apoplast, Zn can be loaded into xylem vessels for later translocation (Axelsen and Palmgren, 2001; Baxter et al., 2003; Eren and Argüello, 2004). Among the members of the HMA family, HMA2 is the only one induced by $\mathrm{Zn}$ deficiency (van de Mortel et al., 2006), however, only the double hma2/hma4 mutant shows decreased $\mathrm{Zn}$ concentration in shoots. The long-distance $\mathrm{Zn}$ transport is also mediated by the PLANT CADMIUM RESISTANCE 2 (PCR2) protein, which is a Zn symplast efflux transporter expressed in the epidermis and xylem parenchyma. It functions in the $\mathrm{Zn}$ removal from the roots in a mechanism independent of HMA4 and HMA2 proteins (Song et al., 2010).

$\mathrm{Zn}$ transporters mediate the mobilization of free $\mathrm{Zn}$ or $\mathrm{Zn}$ chelated to ligands as glutathione, citrate, and oligopeptides such as nicotianamine (NA) (Krężel and Maret, 2016). For instance, members of the YELLOW STRIPE-LIKE (YSL) protein family are involved in the metal-NA complex transport. Within shoots, the double ysllysl3 mutant fails to remobilize Zn from senescing leaves into seeds (Waters et al., 2006). The $\mathrm{Zn}$ phloem transport is still poorly investigated, and probably more genes, apart from YSL1 and YSL3, are involved in this process. 
Several proteins involved in $\mathrm{Zn}$ transport are also able to bind to other divalent cations, such as Cadmium (Cd), Cobalt (Co), Cu, Fe, Mn, Nickel (Ni), and Lead (Pb). (Henriques et al., 2002; Eren and Argüello, 2004; Puig and Peñarrubia, 2009; Sinclair and Krämer, 2012; Milner et al., 2013). The Irving-William series of divalent metal ions $(\mathrm{Cu}>\mathrm{Zn}>\mathrm{Ni}>\mathrm{Co}>\mathrm{Fe}>\mathrm{Mn}>\mathrm{Mg}>\mathrm{Ca})$ shows that micronutrients, such as $\mathrm{Cu}$ and $\mathrm{Zn}$ have a higher affinity than macronutrients, such as $\mathrm{Mg}$ and Calcium (Ca) to a model chelate (Williams, 1984). The overlapping substrate specificities, interactions and relationships among elements can cause large disequilibria in the full plant ionome due to the deficiency of just one of the elements (Huang et al., 2000; Graham, 2008; Nichols et al., 2012; Nishida et al., 2015).

\section{Arabidopsis the model plant for research}

Arabidopsis thaliana (Arabidopsis) is a very useful organism for the discovery and study of novel gene functions. Arabidopsis is the favourite model organism for plant research (Koornneef and Meinke, 2010). Some of the reasons are: its short life cycle, small size, selfpollination, production of a large number of seeds, ease of crossing (Meinke et al., 1998), small size of the genome (Somerville, 1999; The Arabidopsis Genome, 2000), easy and high efficient plant transformation (Clough and Bent, 1998), and large natural variation (Koornneef et al., 2004). A large number of Arabidopsis resources are available for research, and there is an active exchange of stocks among researches (Koornneef and Meinke, 2010). Arabidopsis stock centres include collections of natural accessions and single mutant lines, available to be ordered. In addition, databases and online tools with sequences information, gene expression, proteinprotein interaction, gene networks, gene ontology among others are available to the public (Provart et al., 2016). Arabidopsis intrinsic characteristics, ease of manipulation and availability of resources facilitate researches work at the moment to formulate hypotheses, design experiments and interpret results.

Arabidopsis is widely used to implement and optimise new technologies. It has been extensively used in forward genetics by the generation of randomly mutagenized populations to identify mutants of interest (Page and Grossniklaus, 2002). In reverse genetics, the use of transferred DNA (T-DNA) has been a unique tool for gene functional studies (Page and Grossniklaus, 2002; Meinke et al., 2003). In cell biology, the convergence of Arabidopsis, microscopy and fluorescent proteins has been essential to fulfil genes functional analyses (Tian et al., 2004; Sappl and Heisler, 2013). The high level of variation among Arabidopsis natural accessions (Koornneef and Meinke, 2010) is exploited for the identification of causal polymorphisms. This 
can be done for instance by biparental populations or Genome-Wide Associations (GWA) approaches (Nordborg and Weigel, 2008). The GWA approach overcomes the problems of generating a segregating population and the limited resolution of biparental populations (Kim et al., 2007; Nordborg and Weigel, 2008).

Knowledge generated in Arabidopsis has illuminated the fundamentals of plant biology and help to improve properties of crops (Koornneef and Meinke, 2010; Provart et al., 2016). Several plant mechanisms, including interaction among genes, regulatory loops, biosynthetic pathways of phytohormones, plant immunity, sensing and response to abiotic stress have been discovered due to the research conducted in Arabidopsis (Provart et al., 2016).

\section{The scope of the thesis}

The main aim of this thesis is to increase the knowledge of $\mathrm{Zn}$ deficiency response in the model plant Arabidopsis thaliana. Chapter 2 describes the functional analyses of $\mathrm{Zn}$ transporters induced as a response to $\mathrm{Zn}$ deficiency. The gene expression of these transporters is mapped to their location in the root at the cellular level and evaluated through time during $\mathrm{Zn}$ deficiency. In addition, the effect of loss of function mutants of $\mathrm{Zn}$ transporters is analysed based on the $\mathrm{Zn}$ distribution in roots and the sensitivity to $\mathrm{Zn}$ deficiency.

After showing in Chapter 2, Zn transporters that belong to $\mathrm{Zn}$ deficiency response, I searched for new players involved in $\mathrm{Zn}$ homeostasis. In Chapter 3, I describe a forward genetic approach to search for regulators of the $\mathrm{Zn}$ deficiency response cascade. A mutation in the $\mathrm{N}$ ALPHA-TERMINAL ACETYLTRANSFERASE 25 (NAA25) gene, involved in protein acetylation, affected several pleiotropic traits including aberrant regulation of the $\mathrm{Zn}$ deficiency response, biotic defence response and enhanced susceptibility to Fe deficiency.

In Chapter 4, a different approach is taken to find new Zn deficiency players. In this case, the ionome natural variation in 350 natural accessions of Arabidopsis grown under normal and low Zn supply was used in a Genome-Wide Association (GWA) analysis to identify significant loci associated with micronutrients concentrations. Several loci were associated and candidate genes within those loci were selected to validate their role in $\mathrm{Zn}$ deficiency tolerance. T-DNA insertion lines disrupting the sequence of the candidate genes showed significant changes in $\mathrm{Cu}, \mathrm{Fe}, \mathrm{Mn}$, Molybdenum (Mo), and/or Zn concentration due to $\mathrm{Zn}$ deficiency. 
Chapter 5, was the direct result of the GWAS in Chapter 4. In this Chapter, the functional analyses of a cluster of tandemly arrayed HEAVY METAL-ASSOCIATED ISOPRENYLATED PLANT PROTEINS (HIPP) genes are described. The functional analyses provided information about the expression regulation, interaction with transcription regulators, and tolerance to adverse $\mathrm{Zn}$ and $\mathrm{Fe}$ supply due to the loss function of single HIPPS.

Finally, in Chapter 6, I discuss the main results obtained in my thesis research, their importance for the knowledge of $\mathrm{Zn}$ homeostasis, and future research paths to take this research further. 




\section{Chapter 2}

\section{Uncovering Zn transporters induced by Zn deficiency}

Valeria Ochoa Tufiño ${ }^{1,2}$, Maria Almira Casellas ${ }^{1}$, Aron van Duynhoven ${ }^{1}$ and Mark G.M. Aarts $^{1}$

1) Laboratory of Genetics, Wageningen University, Wageningen, The Netherlands

2) Current affiliation: Departamento de Ciencias de la Vida, Universidad de las Fuerzas Armadas ESPE, Sangolquí, Ecuador

In preparation for submission 


\begin{abstract}
Plants are sessile organisms which have developed several strategies to cope with variable nutrients concentrations in soils to avoid either toxicity or nutrients deficiency. One of the most widespread micronutrient deficiencies in soils is $\mathrm{Zn}$ deficiency. To cope with $\mathrm{Zn}$ deficiency, plants adjust their $\mathrm{Zn}$ homeostasis by inducing the expression of $\mathrm{Zn}$ transporters. In order to learn more about the role of $\mathrm{Zn}$ transporters induced by $\mathrm{Zn}$ deficiency, we performed functional analyses on them. We followed their gene expression induction through time by qRT-PCR, mapped their cell layer expression in roots by using their promoter sequenced fused to YFP, and determined the effects of their loss of function by using single and double T-DNA insertion mutants. The $\mathrm{Zn}$ transporter genes' expression induction starts after six hours of exposure to $\mathrm{Zn}$ deficiency in roots and at day four in shoots, and the highest induction for most of the $\mathrm{Zn}$ transporters, in roots and shoots, is at day twelve. The $\mathrm{Zn}$ transporter ZIP1 gene expresses in the endodermis and stele, ZIP3 and ZIP5 express in the epidermis and cortex, IRT3 expresses from the epidermis to stele and ZIP11 and HMA2 in xylem parenchyma cells. The loss of function of single $\mathrm{Zn}$ transporters does not cause a significant effect in the overall plant performance when compared to wild-type plants. However, the $\mathrm{Zn}$ distribution in the root is disturbed in single mutants. Among double mutants, the zip3/zip5 showed the highest sensitivity to $\mathrm{Zn}$ deficiency and was unable to uptake $\mathrm{Zn}$ during short periods of time. The lack of strong $\mathrm{Zn}$ deficient phenotypes in single mutants suggests that several $\mathrm{Zn}$ transporters have redundant functions, especially for the case of ZIP3 and ZIP5.
\end{abstract}




\section{Introduction}

$\mathrm{Zn}$ is an essential micronutrient for life, being the second most abundant transition element in all organisms. Zn deficiency in plants causes symptoms like stunted growth, chlorosis, reduced yield, among others (Cakmak, 2008; Alloway, 2009; Marschner and Marschner, 2012; Yruela, 2013; Mattiello et al., 2015). Luckily, plants are able to sense the shortage of Zn supply and adjust Zn homeostasis. The transcription factors BASIC REGION-LEUCINE ZIPPER 19 and 23 (bZIP19 and bZIP23) induce the expression of genes, among them some $\mathrm{Zn}$ transporter genes, to increase the $\mathrm{Zn}$ uptake capacity, enabling the plant to adapt to $\mathrm{Zn}$ deficiency stress (Assunção et al., 2010; Assunção et al., 2013). The main known Zn transporters belong to the families of the ZRT-IRT-like proteins (ZIP), heavy-metal ATPases (HMA), and cation diffusion facilitators (CDFs) (Eren and Argüello, 2004; Kobae et al., 2004; Eide, 2006; Sinclair and Krämer, 2012). Arabidopsis encodes fifteen ZIPs, most of them have been suggested to transport divalent cations from extracellular space or organelle lumen into the symplast (Eide, 2006). Several members of the ZIP family are associated with Zn transport due to differential gene expression, yeast functional complementation, and sequence similarity (Grotz et al., 1998; van de Mortel et al., 2006; Milner et al., 2013). Zn deficiency strongly induces gene expression in plants of ZIP1, ZIP3, ZIP4, ZIP5, ZIP9, ZIP10, ZIP12, and IRT3 and in a less degree ZIP2 and ZIP11 (van de Mortel et al., 2006; Assunção et al., 2010; Campos, 2015). The coding sequences of the Arabidopsis genes ZIP1, ZIP2, ZIP3, ZIP4, ZIP7, ZIP11, ZIP12, IRT1, IRT2, and IRT3 complement the growth defect of zrt1zrt2, a yeast mutant defective in Zn uptake (Grotz et al., 1998; Korshunova et al., 1999; Vert et al., 2001; Lin et al., 2009; Assunção et al., 2010; Milner et al., 2013). Proteins as IRT3 can transport Fe as well (Lin et al., 2009), ZIP1 and ZIP3 are highly specific for Zn transport, but $\mathrm{Zn}$ transport can be inhibited by increased concentrations of $\mathrm{Mn}, \mathrm{Co}, \mathrm{Fe}, \mathrm{Cd}$ or $\mathrm{Cu}$ (Grotz et al., 1998). The gene expression response to Zn deficiency and the specificity for Zn as substrate strongly supports that ZIP1, ZIP3, ZIP4, and IRT3 transport Zn especially during Zn deficiency stress.

The CDFs and P1B ATPases transport cations from the symplast to the outside of the cell or into subcellular compartments. In plants, CDFs are usually called MTP (Metal Tolerance Proteins) (Paulsen and Saier Jr, 1997; Montanini et al., 2007). MTP1 and MTP3 are tonoplast localized and mediate the $\mathrm{Zn}$ efflux from the symplast to the vacuole (Kobae et al., 2004; Desbrosses-Fonrouge et al., 2005; Arrivault et al., 2006). The closest homologue of MTP1 and 
MTP3 is MTP2 (Dräger et al., 2004) which is the only member of the MTP family induced by Zn deficiency (van de Mortel et al., 2006).

The P1B ATPases are cation transporters energized by hydrolysis of ATP. The sequence of the HEAVY METAL ATPASE 3 (HMA3) complements the $y c f 1$ yeast mutant $\mathrm{Cd} / \mathrm{Pb}$-hypersensitive and the Arabidopsis HMA3 loss-of-function mutant is sensitive to high concentrations of $\mathrm{Zn}$ and $\mathrm{Cd}$. HMA3 is located in the tonoplast and is likely involved in $\mathrm{Zn}$ detoxification by vacuolar sequestration (Morel et al., 2009). HMA4 rescues the zntA Escherichia coli mutant sensitive to high Zn (Mills et al., 2003) and Arabidopsis HMA4 loss-of-function mutant accumulates less $\mathrm{Zn}$ in shoots than wild-type plants (Hussain et al., 2004). The HMA2 ATPase activity is activated by $\mathrm{Zn}$ and $\mathrm{Cd}$ and, to a lesser extent by $\mathrm{Pb}, \mathrm{Ni}, \mathrm{Cu}$, and $\mathrm{Co}$ (Eren and Argüello, 2004) and its expression is induced by $\mathrm{Zn}$ deficiency (van de Mortel et al., 2006). HMA2 and HMA4 are expressed in the vasculature of roots, stems and leaves and promotes $\mathrm{Zn}$ translocation from roots to shoots (Hussain et al., 2004) (Sinclair et al., 2007). The double mutant hma2/hma4 significantly decreases $\mathrm{Zn}$ concentration in shoots (Hussain et al., 2004).

Zinc can be transported in complex with organic molecules, such as citrate malate, histidine or nicotianamine (NA) (Beneš et al., 1983; Anderegg and Ripperger, 1989; Scholz et al., 1992). Zn-NA complex can be transported across membranes by members of the Yellow Stripe-Like (YSL) family of oligopeptide transporters, eg. YSL1 and YSL3 (Waters et al., 2006). The ZnNA complex facilitates the $\mathrm{Zn}$ mobilization, by preventing $\mathrm{Zn}$ precipitation or binding with cell walls (Deinlein et al., 2012).

The $\mathrm{Zn}$ shoot supply depends on how effectively roots obtain $\mathrm{Zn}$ and make it available for translocation to other parts of the plant. In the roots, nutrients move radially through epidermis, cortex, and endodermis to enter the stele, where nutrients are loaded into the xylem. This transport can be apoplastic, i.e. by passive transport through cell walls and intercellular spaces, or symplastic, mediated by influx carriers (transporters) and subsequently from cell-to-cell through plasmodesmata, or in a combination of both, the coupled pathway, which combines influx and efflux carriers to transport nutrients from one cell to the other. Transport across the endodermis has to occur symplastically since Casparian strip prevents most of the apoplastic transport (Clarkson, 1993; Tester and Leigh, 2001; Geldner, 2013; Barberon and Geldner, 2014). 
Previous studies of $\mathrm{Zn}$ transporters have not examined in detail the $\mathrm{Zn}$ transport in roots. With the aim of filling this research gap, we followed Zn fluxes using Zinpyr-1 in the roots of wildtype plants and loss-of-function mutants of the most well-known $\mathrm{Zn}$ transporters. Based on promoter studies and gene expression studies, we show the root cell layer(s) where each transporter is expressed and how their expression levels change over time. Moreover, we analyzed the general effect on plants of single and double loss-of-function $\mathrm{Zn}$ transporter mutants. These approaches provided us with information on how each of the studied $\mathrm{Zn}$ transporters contribute to the $\mathrm{Zn}$ uptake and transfer.

\section{Materials and Methods}

\section{Plant material}

T-DNA insertion lines for any of the Arabidopsis genes studied were ordered from NASC (www.arabidopsis.info/BasicForm) (Supplemental Table S1). Arabidopsis accession Columbia was used for transformation and as a wild-type control.

\section{Growing conditions}

Prior to germination, seeds were stratified for three days at $4^{\circ} \mathrm{C}$ in the dark. For propagation and genotyping, plants were grown in a greenhouse set at a $16 / 8 \mathrm{~h} \mathrm{light/dark} \mathrm{cycle} \mathrm{at} 20 / 18^{\circ} \mathrm{C}$ day/night temperatures and 70\% humidity. For Zn deficiency treatment, plants were grown hydroponically, in a climate-controlled growth chamber set at a 12/12 h light/dark cycle at $20 / 15^{\circ} \mathrm{C}$ day/night temperatures and $70 \%$ humidity. Seeds were surface-sterilized using vaporphase seed sterilization (Clough and Bent, 1998) and sown on $0.55 \%$-agar-filled tubes in trays containing a modified half-strength Hoagland's nutrient solution (Schat et al., 1996). During ten days, plants grew in a fully supplemented medium (containing $2 \mu \mathrm{M} \mathrm{ZnSO}_{4}$ ), after that, plants were either treated with $\mathrm{Zn}$ deficiency (no $\mathrm{Zn}$ added to the medium) or continued to grow at fully supplemented medium. For the $\mathrm{Zn}$ uptake experiment, plants were grown for 15 days

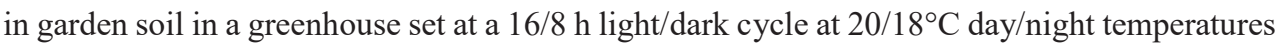
and $70 \%$ humidity. After that, plants were grown hydroponically, in the conditions mentioned above, for 30 days in $\mathrm{Zn}$ deficiency (no $\mathrm{Zn}$ added to the medium). Then, the plants were placed, for three hours in the morning, in a fully supplemented medium (containing $2 \mu \mathrm{M} \mathrm{ZnSO}_{4}$ ). For $\mathrm{Zn}$ tracking, surfaced-sterilized seeds were sown on 9-cm circular plates filled with water in a climate-controlled growth chamber set at a $16 / 8 \mathrm{~h}$ light/dark cycle at $22 / 20^{\circ} \mathrm{C}$ day/night temperatures and 50\% humidity. For gene expression imaging, surfaced-sterilized seeds were 
sown on 12-cm square plates containing 1\%-agar-solidified 0.5 MS medium (pH 5.8). Plants grew either on a fully supplemented medium (containing $15 \mu \mathrm{M} \mathrm{ZnSO}_{4}$ ) or on medium to which no $\mathrm{Zn}$ was added in case of $\mathrm{Zn}$ deficiency treatment. Plants were grown in a climate-controlled

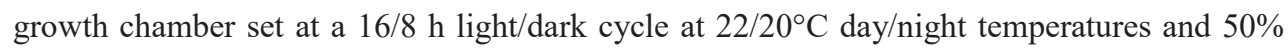
humidity.

\section{Genotyping}

T-DNA insertion lines were genotyped to confirm or select for homozygote T-DNA insertion plants. Oligonucleotide primers were designed using the Salk Institute website (signal.salk.edu/tdnaprimers.2.html). Two PCR reactions were performed per plant, the first with the primers combination left border (LB)-T-DNA border (BP) and the second with the primer combination LB-right border (RP) (Supplemental Table S1).

\section{Zn tracking}

Five- to seven-day-old seedlings were incubated with 5 uM of Zinpyr-1 (Sigma-Aldrich; www.sigmaaldrich) for 3 hours as described in Sinclair et al. (2007). Cell walls were stained with $10 \mathrm{mg} / \mathrm{ml}$ Propidium Iodide (PI) for $30 \mathrm{sec}$. Images were taken with a CLSM (Confocal Laser-Scanning Microscope) Leica SPE DM5500 upright run with the LAS AF 1.8.2 software (Leica; www.leica-microsystems). Zinpyr-1 and PI were excited with a 488-nm solid-state laser. The Zinpyr-1 and PI emission were detected at a bandwidth of 540-550 and 700-800 nm respectively. Images were processed using Image J (Schneider et al., 2012).

\section{Gene expression quantification}

The treatment was applied at 8:00 am and samples were collected $15 \mathrm{~min}, 1$ hour, 3 hours, 6 hours, 12 hours, after treatment in day one, then at 8:00 pm in day two, four and twelve. Full root systems and shoots of three plants were collected for each biological replicate. Three biological replicates were collected in each time point and treatment. Total RNA was extracted with Direct-zol ${ }^{\mathrm{TM}}$ RNA MiniPrep Kit (Zym o Research, www.zym oresearch) and cDNA was synthetized with the iScript cDNA Synthesis Kit (Bio-Rad, www.bio-rad.). Gene expression was quantified by reverse transcriptase quantitative PCR (RT-qPCR) based on the SYBR ${ }^{\circledR}$ Green mix (Bio-Rad, www.bio-rad.). Primers are described in Supplemental Table S2. 


\section{Generation of promoter-NLS-YFP transgenic lines}

A destination vector based on the Invitrogen Gateway technology was constructed to clone the promoter sequences. For this purpose, pFAST-R07 (Shimada et al., 2010) was digested with EcoRI and NruI to remove the eGFP coding sequence. In parallel, synthetic yellow fluorescent protein 2 (SYFP2) (Kremers et al., 2006)was isolated from pCZN633 (Smaczniak et al., 2012) by PCR using primers containing a short linker including an NruI restriction enzyme site and an overlapping sequence with a nuclear localisation signal (NLS) fragment. The NLS was isolated from pGREEN:GW:NLS-GFP (Horstman et al., 2015) by PCR using primers containing a short linker including an overlapping sequence with the sYFP2 fragment. The NLS and sYFP2 fragments were connected with a double joint PCR (Yu et al., 2004) and subsequently digested with EcoRI and NruI. The digested pFAST-R07 and NLS-sYFP2 fragments were ligated resulting in pFAST-R7-VO (Supplemental Figure S1). PCR primers for vector construction are detailed in Supplemental Table S3. The different promoter sequences were isolated from Arabidopsis Col-0 genomic DNA. Promoters were selected to be between $435 \mathrm{bp}$ and $1800 \mathrm{bp}$ depending on the distance with the upstream gene. Promoter PCR fragments were cloned into the pDONOR201 vector and subsequently into the expression vector pFAST-R7-VO by standard Gateway BP and LR reactions. Primers for promoter sequences cloning are detailed in Supplemental Table S4. Arabidopsis plants were transformed by the floral dip method (Clough and Bent, 1998). Transformed seeds (T1 generation) were selected based on the red fluorescence marker expressed in seeds (Shimada et al., 2010).

\section{Gene expression imaging}

Transgenic Arabidopsis T2 seeds were used. Roots from six- to ten-day-old seedlings were stained with $10 \mathrm{mg} / \mathrm{ml}$ PI for $30 \mathrm{~s}$. CSLM images were taken as described above. The $488 \mathrm{~nm}$ solid-state laser was used to excite SYFP2 and PI. The SYFP2 and PI emission were detected at a bandwidth of 530-570 and 700-800 nm respectively. Images were processed using Image J (Schneider et al., 2012).

\section{Metal content analysis}

Root and shoot samples were collected to do a metal content analysis as was described by (Assunção et al., 2003). The metal content of $\mathrm{Zn}$ was measured in an atomic absorption Spectrophotometry. 


\section{Statistical analysis}

Data was $\log 10$ transformed prior to any test. Two-sample t-test, one- and two-way ANOVA and Tukey post hoc tests were performed using Genstat 18th edition (VSN International; www.vsni.co.uk/software/genstat/).

\section{Results}

\section{Zn distribution in Arabidopsis $\mathrm{Zn}$ deficient roots}

We have used the Zn fluorescent dye Zinpyr-1 to visualize the Zn distribution in roots of young (5-7 days old) Arabidopsis Col-0 seedlings. Zinpyr-1 is a fluorescein-based bright fluorescent $\mathrm{Zn}^{+2}$ sensor (Woodroofe et al., 2004), its fluorescence intensity indicates relative levels of $\mathrm{Zn}$ (Sinclair et al., 2007). Zn sufficiency conditions were not applied due to the strong fluorescence in all root layers, which did not allow the differentiation of $\mathrm{Zn}$ distribution patterns. Seedlings are grown in strong $\mathrm{Zn}$ deficiency conditions to force the plants to prioritize their $\mathrm{Zn}$ distribution. The presence of $\mathrm{Zn}$ is examined in the whole root, from the root tip to the transition zone (Figure 1). The root tip, lateral root primordia, lateral root tips, and the transition zone show the highest Zinpyr-1 fluorescence, while the elongation zone shows the least fluorescence. In the differentiation zone, the Zinpyr-1 fluorescence is concentrated in the stele and endodermis. In addition, within epidermal and cortex cells, the Zinpyr-1 fluorescence intensity shows a gradient, being higher in the direction of the transition zone than in the direction of the root tip.

In five-day-old seedlings, the closer to the transition zone, the more intense the Zinpyr-1 fluorescence is in the stele (Figure 1A). In six-day-old seedlings, fluorescence in the stele close to the transition zone (Figure 1B), is lower compared to five-day-old seedlings. In seven-dayold seedlings, the fluorescence concentrated in the stele just until half of the root (Figure 1C). From halfway the root to the transition zone, the Zinpyr-1 fluorescence is spread along the epidermis, cortex, and endodermis (Figure 1D-F). Therefore, the Zinpyr-1 fluorescence in the stele close to the transition zone decreases when the tissue becomes older. In five-, six-, and seven-day-old seedlings, the high Zinpyr-1 fluorescence is consistently high in root zones with dividing cells, suggesting $\mathrm{Zn}$ tends to locate to growing tissues. 


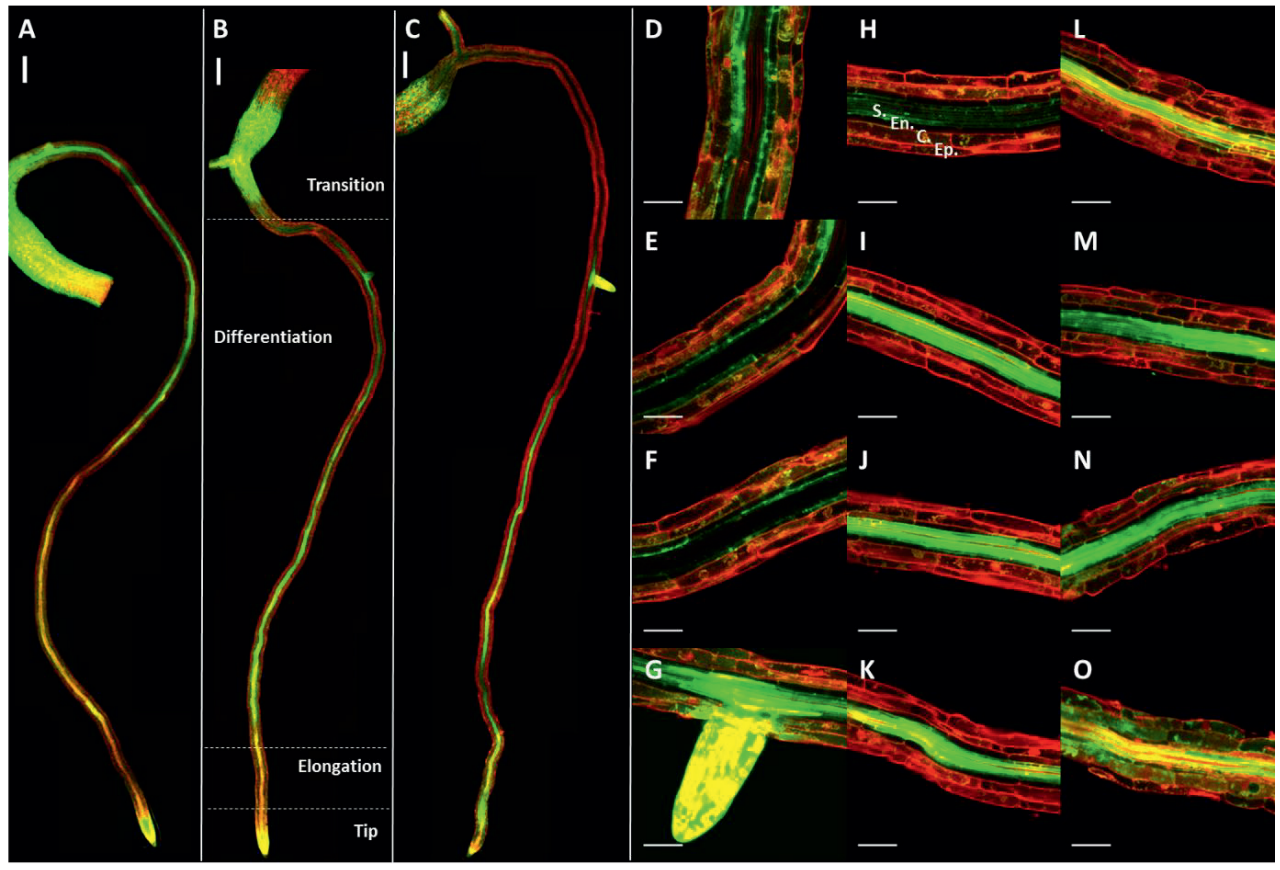

Figure 1. Confocal laser-scanning microscope images of $\mathrm{Zn}$ distribution in Arabidopsis roots. $\mathrm{Zn}$ deficient Arabidopsis Col-0 roots incubated with Zinpyr-1 to detect Zn (A) in five-, (B) six-or (C) sevenday-old plants covering the root between $(D)$ the transition zone and the (O) root tip. Zinpyr-1 complexed to Zn fluoresces green, while propidium iodide bound to cell wall or DNA fluoresces red. Root cell layers can be differentiated from the outside to inside epidermis (Ep.), cortex (C.), endodermis (En.) and stele (S)) as described in (H). The scale bars indicate $200 \mu \mathrm{m}$ in A-C, and $50 \mu \mathrm{m}$ in D-O.

After elucidating the $\mathrm{Zn}$ distribution in roots of wild-type plants, we analysed the $\mathrm{Zn}$ distribution in roots of $\mathrm{Zn}$ transporters loss-of-function mutant plants. Our target were the $\mathrm{Zn}$ transporter genes ZIP1, ZIP3, ZIP5, IRT3, and YSL3, because their expression in roots is induced by Zn deficiency (van de Mortel et al., 2006) thus, we used T-DNA insertion lines of these genes. Of all mutants examined, the five-days-old zip3 and zip5 mutants show the strongest difference in Zn distribution compared to wild type (Supplemental Figure S2). Both mutants lack the high Zinpyr-1 fluorescence in lateral root primordia and in the stele, similar to the seven-day-old wild-type seedlings (Figure 2). In these mutants the Zinpyr-1 fluorescence is seen particularly in pericycle cells within the stele, but not in the whole stele as in wild type. In the differentiation zone close to the transition zone, the Zinpyr-1 fluorescence is seen in cortex and epidermis in zip 3 mutants and in cortex only in zip 5 mutants (Figure 2). The Zinpyr- 
1 fluorescence pattern in five-day-old zip3 and zip5 seedlings resembles seven-day-old wildtype seedlings, suggesting more retention and less translocation to shoots in these mutants.

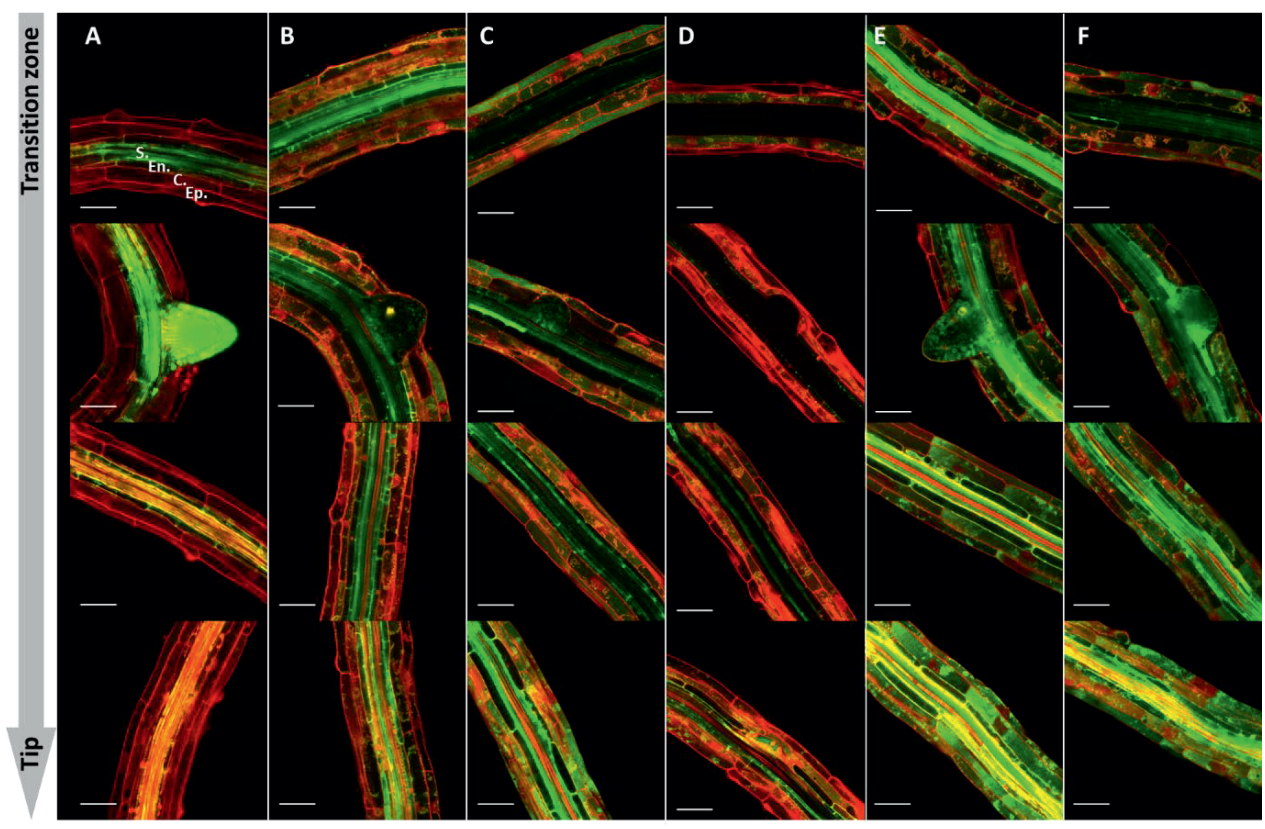

Figure 2. Confocal laser scanning microscope images of Zn deficient Arabidopsis roots. Sections of different genotypes incubated with Zinpyr-1 to detect Zn. Zinpyr-1 complexed to Zn fluoresces green, while propidium iodide bound to cell wall or DNA fluoresces red. Sections from the younger (bottom) to the older (up) regions of the root differentiation zone of five-day-old (A) Col-0 wild-type, (B) zip1, (C) zip3, (D) zip5, (E) irt3 and (F) ysl3 plants. The cell layers can be differentiated from the outside to inside ((epidermis (Ep.), cortex (C.), endodermis (En.) and stele (S.)) as described in (A) top image. The scale bars indicate $50 \mu \mathrm{m}$.

The Zinpyr-1 fluorescence in zipl mutant shows similar intensity in all root layers, while in wild-type roots the fluorescence intensity is higher in the stele (Figure 2 and Supplemental Figure S2). In addition, Zinpyr-1 fluorescence, in zip1, is not particularly intense in lateral root primordia, as it is wild-type roots. The Zinpyr-1 fluorescence intensity pattern was similar between the ysl3 and zip1 mutants. The five-day-old seedlings of the zip1, zip3, zip5 and ysl3 mutants showed a common Zinpyr-1 fluorescence pattern of less intensity in the stele and lateral primordia compared to the wild type. There is no difference in the Zinpyr-1 fluorescence pattern distribution between the irt3 mutant and wild type, while the intensity seems higher in irt3 mutant than in wild type. 


\section{Gene expression patterns in $\mathrm{Zn}$ deficiency}

We determ ined $\mathrm{Zn}$ transporter genes' expression induction upon $\mathrm{Zn}$ deficiency in order to examine their spatial and timely coordination. The Zn deficiency or control treatment was applied to 15-day-old plants. The treatment started at 8:00 am on day one and samples were collected at different time points, ranging between fifteen minutes and twelve days of treatment. On day one, the last sample was collected at 8:00 pm, samples from day two, four and twelve were also collected at 8:00 pm.
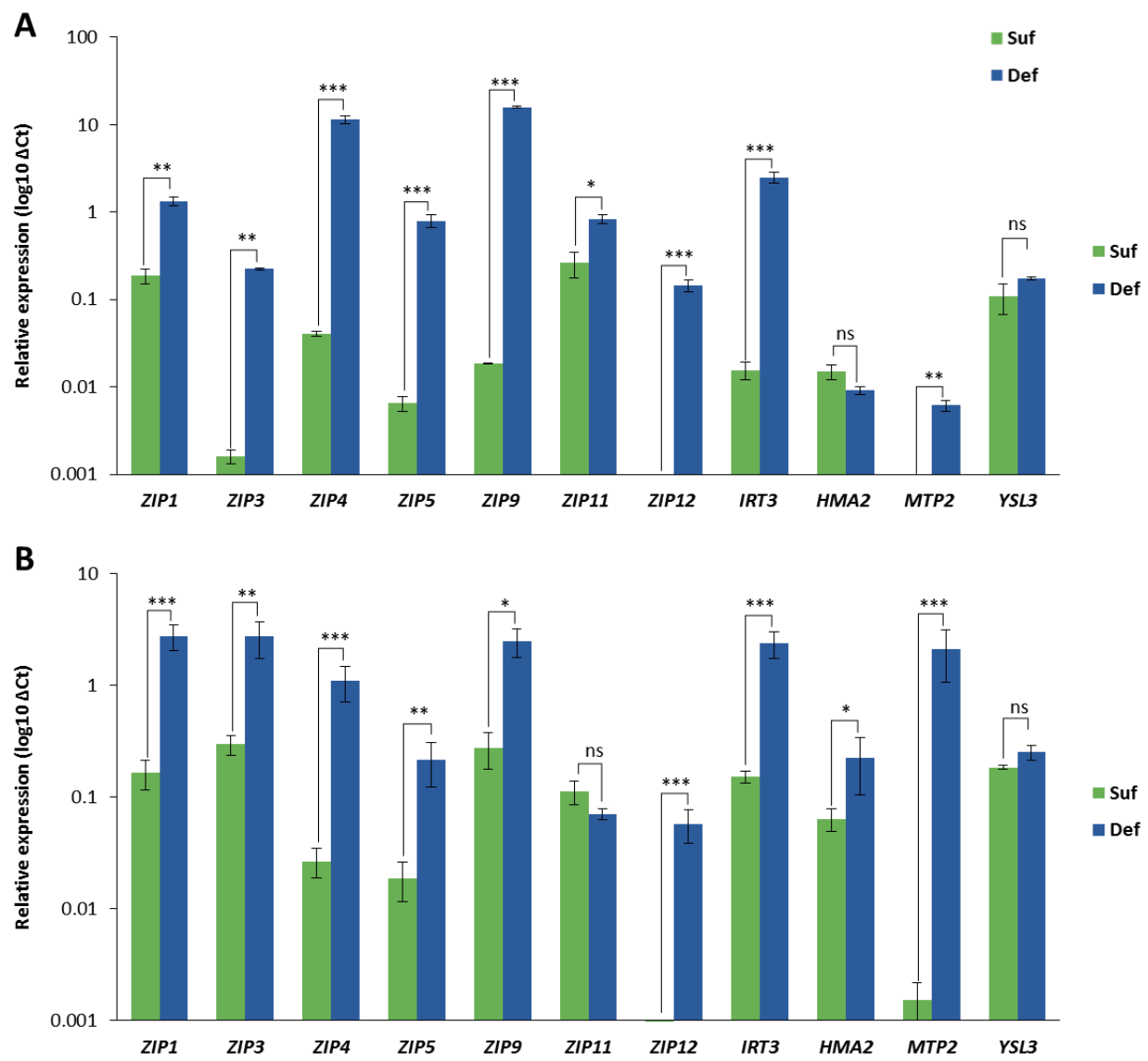

Figure 3. Gene expression of Zn transporters in shoots and roots of Arabidopsis Col-0 after twelve days of Zn deficiency treatment. Gene expression relative to At5g25760 and AT2G28390 (A) in shoots and (B) roots of plants grown for ten days in fully supplied medium and then twelve days in either Zn deficiency (Zn def: blue bars) or Zn sufficiency (Zn suf: green bars). Mean $\pm S E, n=3$ of 3 pooled plants. Statistical significance was determined by Student's t test ${ }^{*} p<0.05,{ }^{* *} p<0.01$, and $* * * p<$ $0.001)$. 
The highest level of gene expression induction was detected at day twelve of exposure to $\mathrm{Zn}$ deficiency, for most of the genes (Table 1 and Supplemental Figure S3). At day twelve, ZIP1, ZIP3, ZIP4, ZIP5, ZIP9, ZIP11, ZIP12, IRT3, and MTP2 in shoots (Figure 3A), and ZIP1, ZIP3, ZIP4, ZIP5, ZIP9, ZIP12, IRT3, MTP2, and HMA2 in roots (Figure 3B) are all significantly higher expressed in response to $\mathrm{Zn}$ deficiency when compared to plants grown in Zn sufficiency. The highest expressed genes upon Zn deficiency treatment are ZIP4 and ZIP9 in shoots and ZIP1, ZIP3, ZIP4, ZIP9, IRT3, and MTP2 in roots at day twelve days of exposure to $\mathrm{Zn}$ deficiency. Instead, the highest fold induction by $\mathrm{Zn}$ deficiency at day twelve was detected for ZIP9 and ZIP12 in shoots and ZIP12 and MTP2 in roots was, this effect was largely due to their low expression under Zn sufficiency.

Some genes did not have their highest level of induction at day twelve. In shoots, the highest induction of HMA2 was after 12 hours and of YSL3 was at day four of exposure to $\mathrm{Zn}$ deficiency, however, their induction does not reach a 3 fold increase. In the roots, the highest induction of YSL3 was at day four of exposure to Zn deficiency. And the expression of ZIP11 is not affected by $\mathrm{Zn}$ deficiency in roots (Table 1 and Supplemental Figure S3). Apart from day twelve when the induction of most of genes peak, the expression profile of $\mathrm{Zn}$ transporters changes over time. While in the shoots, there was no significant increase of expression until day four, when ZIP1, ZIP4, ZIP5, ZIP12, IRT3, HMA2, MTP2, and YSL3 significantly increased their expressions. Instead in roots, after six hours of deficiency ZIPI and ZIP4 significantly increase their expression, with a three-fold change, after twelve hours ZIP3, ZIP5 and IRT3, at thirty-six hours ZIP1, ZIP3, and IRT3, and at day four ZIP1, ZIP4, and YSL3. Both in the roots and shoots, most of the genes follow the pattern of a first low peak of gene expression induction at twelve hours, a drop of induction after thirty six hours, a second low peak of gene expression induction at day four and a final high peak of gene expression induction at day twelve (Table 1).

\section{Zn transporters are expressed in different cell layers of the root}

The ZIP family of transporters is able to transport cations (mostly Fe and $\mathrm{Zn}$ and some related ions) into the symplast, while the HMA and CDF family transporters are mostly thought to be involved in the export of cations from the symplast, either to the intercellular space, or to the vacuole or other organelles (Eren and Argüello, 2004; Kobae et al., 2004; Eide, 2006; Sinclair and Krämer, 2012). We decided to study the root cell-specific expression of several ZIP, HMA and $\mathrm{CDF}$ genes suggested to be implicated in $\mathrm{Zn}$ transport. For that purpose, we generated 
transgenic Arabidopsis lines expressing a nuclear localized yellow fluorescent protein (NLSSYFP2) of which transcription is controlled by the promoters of ZIP1, ZIP3, ZIP5, ZIP9, ZIP10, ZIP11, ZIP12, IRT3, MTP2, YSL3, and HMA2. As a rule of thumb, we considered promoter regions of at most $1800 \mathrm{bp}$ upstream of the predicted coding sequence start codon, but depending on the position of the upstream gene, this could be shorter. ZIP11 has the shortest promoter region, corresponding to $435 \mathrm{bp}$. Three ZIP genes share their promoter regions with other genes: 1) ZIP1 with an auxin-responsive gene (AT3G12760) (Biswas et al., 2007); 2) ZIP9 with a sulfolipid synthetase (AT4G33030) (Essigmann et al., 1998; Sanda et al., 2001); and 3) ZIP10 with a gene of unknown function (AT1G31270)

Table 1. Fold changes of gene expression of Zn transporters through time. Fold change means of gene expression relative to At5g25760 and AT2G28390 after increasing times (from 0.25 hours (h) to 12 days (d)) of exposure to Zn deficiency, when compared to plants grown at Zn sufficiency. Colours indicate the highest fold change (red), middle (while) and the lowest fold change (blue) among time points within each gene. Darker numbers indicate statistical significant gene expression induction when comparing between Zn deficiency and Zn sufficiency grown plants at each time points per gene. Statistical significance was determined by Student's t test $(p<0.05)$.

\begin{tabular}{|c|c|c|c|c|c|c|c|c|c|c|c|}
\hline Shoot & ZIP1 & ZIP3 & ZIP4 & ZIP5 & ZIPg & ZIP11 & ZIP12 & IRT3 & HMA2 & MTP2 & YSL3 \\
\hline 0.25 & 0.9 & 0.8 & 0.8 & 0.6 & 0.9 & 0.8 & 0.6 & 0.7 & 1.0 & 0.5 & 0.8 \\
\hline $1 \mathrm{~h}$ & 0.7 & 0.9 & 1.4 & 0.4 & 0.7 & 0.8 & 0.1 & 0.7 & 0.6 & 0.8 & 0.8 \\
\hline $3 \mathrm{~h}$ & 1.0 & 0.7 & 1.1 & 0.7 & 0.9 & 1.0 & 0.6 & 1.2 & 0.8 & 1.3 & 0.9 \\
\hline $6 \mathrm{~h}$ & 1.4 & 1.4 & 1.5 & 1.3 & 0.7 & 0.9 & 0.9 & 1.8 & 2.1 & 0.6 & 1.1 \\
\hline $12 \mathrm{~h}$ & 1.5 & 1.2 & 1.3 & 1.4 & 1.5 & 1.1 & 3.0 & 2.5 & 1.2 & 1.1 & 1.3 \\
\hline $36 \mathrm{~h}$ & 0.4 & 0.8 & 0.8 & 0.7 & 0.5 & 0.4 & 0.5 & 1.0 & 0.2 & 0.7 & 1.2 \\
\hline $4 \mathrm{~d}$ & 2.0 & 0.7 & 1.4 & 2.3 & 1.4 & 1.4 & 5.8 & 4.5 & 1.7 & 2.2 & 2.8 \\
\hline $12 \mathrm{~d}$ & 7.4 & 144 & 285 & 125.7 & 853.3 & 3.6 & 886.6 & 169.5 & 0.6 & 8.8 & 1.9 \\
\hline Root & ZIP1 & ZIP3 & ZIP4 & ZIP5 & ZIP9 & ZIP11 & ZIP12 & IRT3 & HMA2 & MTP2 & $Y S L 3$ \\
\hline $0.25 \mathrm{~h}$ & 1.1 & 0.7 & 0.9 & 0.8 & 0.8 & 2.3 & 0.3 & 0.6 & 1.2 & 0.3 & 0.6 \\
\hline $1 \mathrm{~h}$ & 2.0 & 0.9 & 1.5 & 0.5 & 1.2 & 2.3 & 3.8 & 1.1 & 0.6 & 0.4 & 0.2 \\
\hline $3 \mathrm{~h}$ & 1.9 & 1.1 & 2.4 & 1.3 & 0.9 & 1.1 & 1.4 & 1.7 & 0.7 & 0.2 & 1.2 \\
\hline $6 \mathrm{~h}$ & 3.3 & 1.6 & 3.1 & 1.6 & 1.0 & 1.7 & 0.9 & 1.9 & 0.7 & 1.2 & 0.4 \\
\hline $12 \mathrm{~h}$ & 2.7 & 2.1 & 3.5 & 3.1 & 0.9 & 1.6 & 4.4 & 5.9 & 1.4 & 0.8 & 1.8 \\
\hline $36 \mathrm{~h}$ & 2.5 & 1.8 & 1.5 & 1.5 & 0.6 & 0.7 & 0.7 & 2.3 & 0.9 & 0.6 & 2.8 \\
\hline $4 d$ & 3.4 & 1.7 & 2.2 & 1.4 & 1.3 & 1.2 & 0.4 & 2.4 & 0.4 & 2.1 & 5.0 \\
\hline $12 \mathrm{~d}$ & 17.3 & 9.3 & 42.3 & 12.0 & 10.1 & 0.7 & 1289.5 & 15.5 & 3.6 & 1833.7 & 1.4 \\
\hline
\end{tabular}



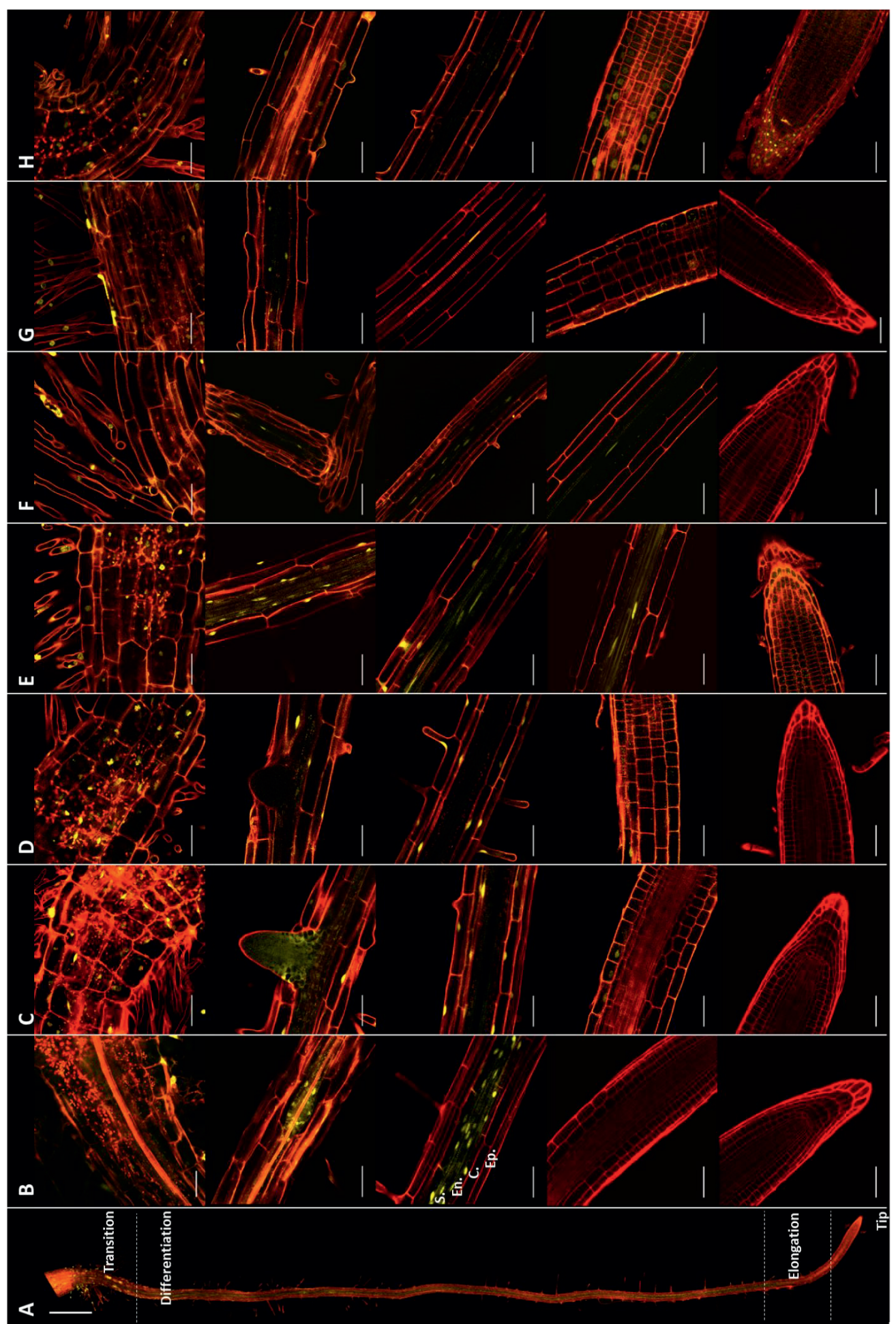


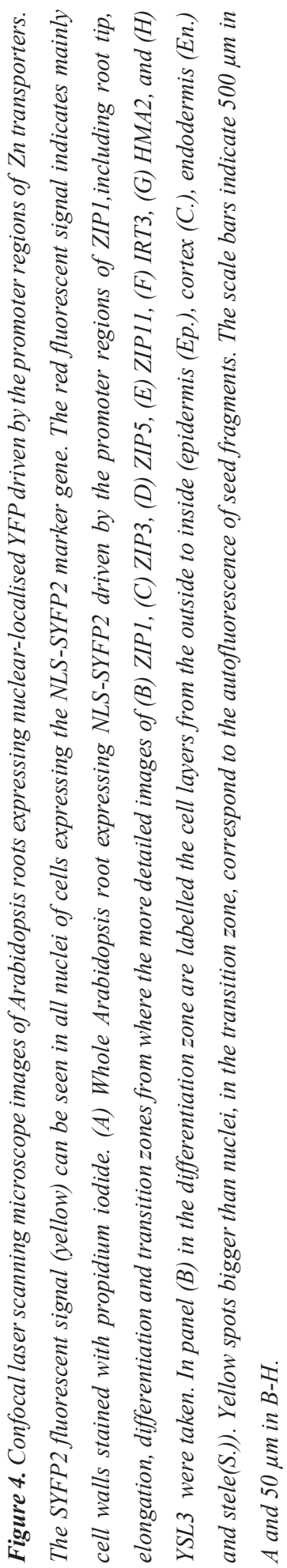


To analyse the cell-specific expression, we examined two independent transgenic lines per construct grown on $\mathrm{Zn}$ deficient vertical agar plates. The two transgenic lines showed the same expression pattern per construct. We used T2 seeds which were either homozygous or heterozygous for the insertion, selected based on the seed red fluorescence (Shimada et al., 2010). For all promoters, except for ZIP10, expression of YFP is detected by confocal microscopy (Figure 4 and Supplemental Figure S4). ZIP1 is expressed in the endodermis, stele, and lateral root primordia. ZIPI is the gene with the lowest expression detected in the transition zone between root and hypocotyl (Figure 4B). ZIP3 and ZIP5 had the same expression pattern, both genes are expressed from the elongation to the transition zone of the root. In the differentiation zone, we can clearly see, ZIP3 and ZIP5 expression in the epidermis and cortex. The only difference in their expression is that ZIP3 is highly expressed in lateral root primordia, and in all cell layers of young lateral roots, while ZIP5 expression was not detected in those cases (Figures 4C and D). ZIP11 is expressed from the elongation to the transition zone. In the young differentiated zone, ZIP11 is expressed in xylem parenchyma cells, but in the older parts of the root the expression shifts to endodermal cells (Figure 4E). This shift occurs abruptly, with hardly any overlap between both ZIPI1 expression sites. The expression of ZIP9 and ZIP12 was only detected in the transition zone, where it was not possible to clearly differentiate cell layers (Supplemental Figure S4). IRT3 is expressed in the root tip, the differentiated zone, and the transition zone. In the young differentiated zone, IRT3 is expressed in the inner stele cells accompanying the xylem parenchyma cells protoxylem vessels. In the older differentiated zone, IRT3 expressed in all cell types from the epidermis to stele. The shift occurs gradually, with overlap between both IRT3 expression patterns (Figure 4F). Like IRT3, HMA 2 is expressed in the inner stele next to xylem vessels, mainly in the young differentiated zone. HMA2 is also expressed in the transition zone. The HMA2 expression in the lateral roots is stronger than in the primary root (Figure 4G). YSL3 is expressed in the root cap, the elongation zone, the differentiated zone, and the transition zone. In the differentiated zone, YSL3 expressed in the epidermis and cortex, however its expression was low and hard to detect (Figure 4H). The expression of MTP2 was only detected in the transition zone, particularly in the root hairs (Supplemental Figure S4).

\section{ZIP Zn transporters are largely redundant in response to $\mathrm{Zn}$ deficiency}

To evaluate the plant response to the loss of one or two ZIP transporters, single and double TDNA insertion lines of these genes are evaluated in response to $\mathrm{Zn}$ sufficiency and $\mathrm{Zn}$ deficiency. In Zn sufficiency, the shoot phenotype of zip1, zip3, zip5, and zip9 look smaller 
than wild-type shoots (Figure 5A and B), but these mutants are not statistically different from the wild type (Figure 5C and D). However, zip3, zip5 and zip9 shoot dry weights are significantly lower than the double mutants zip1/zip3, zip1/zip5, zip9/zip12, and zip9/mtp2. The root dry weight of roots of zip 9 is significantly lower than the double mutants zip1/zip3, zipl/zip5, zip9/zip12, and zip9/mtp2 (Figure 5C and D), which is a similar pattern as the one observed in the shoots. In Zn deficiency, the shoot phenotype of most of the genotypes cannot be distinguished from the wild type. However, the exceptions were the double mutants zip1/zip3 and zip3/zip5, which are more yellow, and with more curly leaves than the wild type, in addition zip3/zip5 is clearly smaller than the wild type (Figure 5C). The shoot dry weight of zip3/zip5 is significantly lower than the wild type and all the other mutants, while the shoot dry weight of zip 1/zip 3 is significantly lower than all the other genotypes except for the wild type, zip 5 and zip9/mtp2. The root dry weight of roots of any of the mutants was different from the wild type. However, zip 9 and zip3/zip5 are significantly lower than zipl/zip5 (Figure 5D).

Because the double mutant zip3/zip5 was the only genotype significantly different from the wild type, we analysed its $\mathrm{Zn}$ uptake and translocation capacity compared to wild type and the single mutants zip3 and zip5. In Zn deficient plants, the shoot Zn concentration of zip3 and zip5 is not different from the wild type, while that of zip3/zip5 is higher than wild type (Figure 6A). The root $\mathrm{Zn}$ concentration of all genotypes tested was similar in $\mathrm{Zn}$ deficiency (Figure 6B). To analyse the $\mathrm{Zn}$ uptake and translocation capacity, Zn deficient plants were supplied $\mathrm{Zn}$ for three hours before harvesting. After these three hours, the Zn concentration significantly increased in shoots and roots of all genotypes except in zip3/zip5. The response was particularly strong in roots where the increase was approximately nine fold in wild type, zip3 and zip5. 


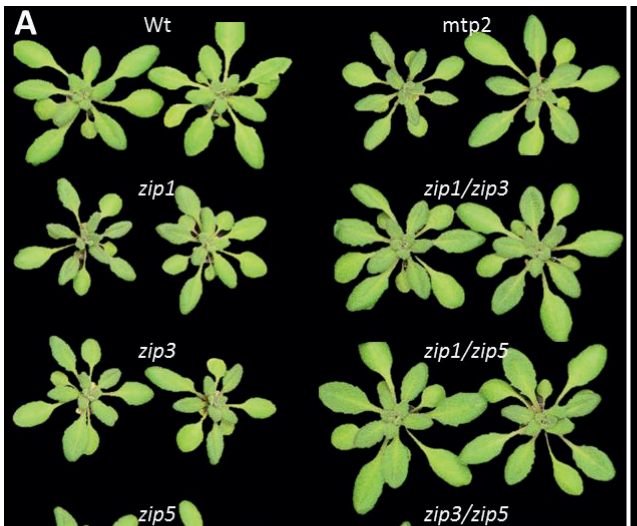

B काष $25^{2 i p 3}$ gesesogs atopo

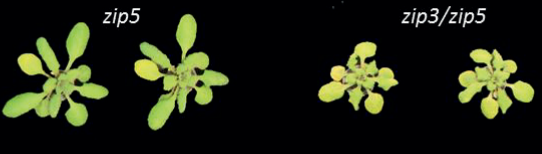

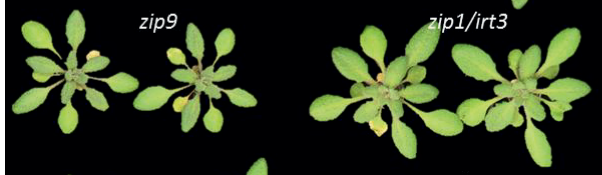

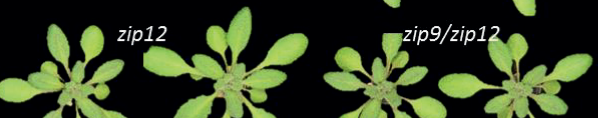
os 055 sis 85

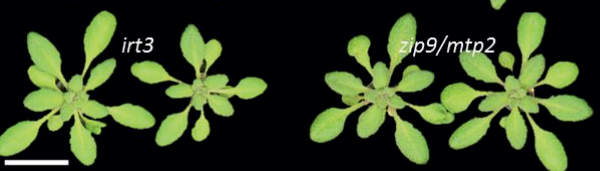
ske in को of if को ats of
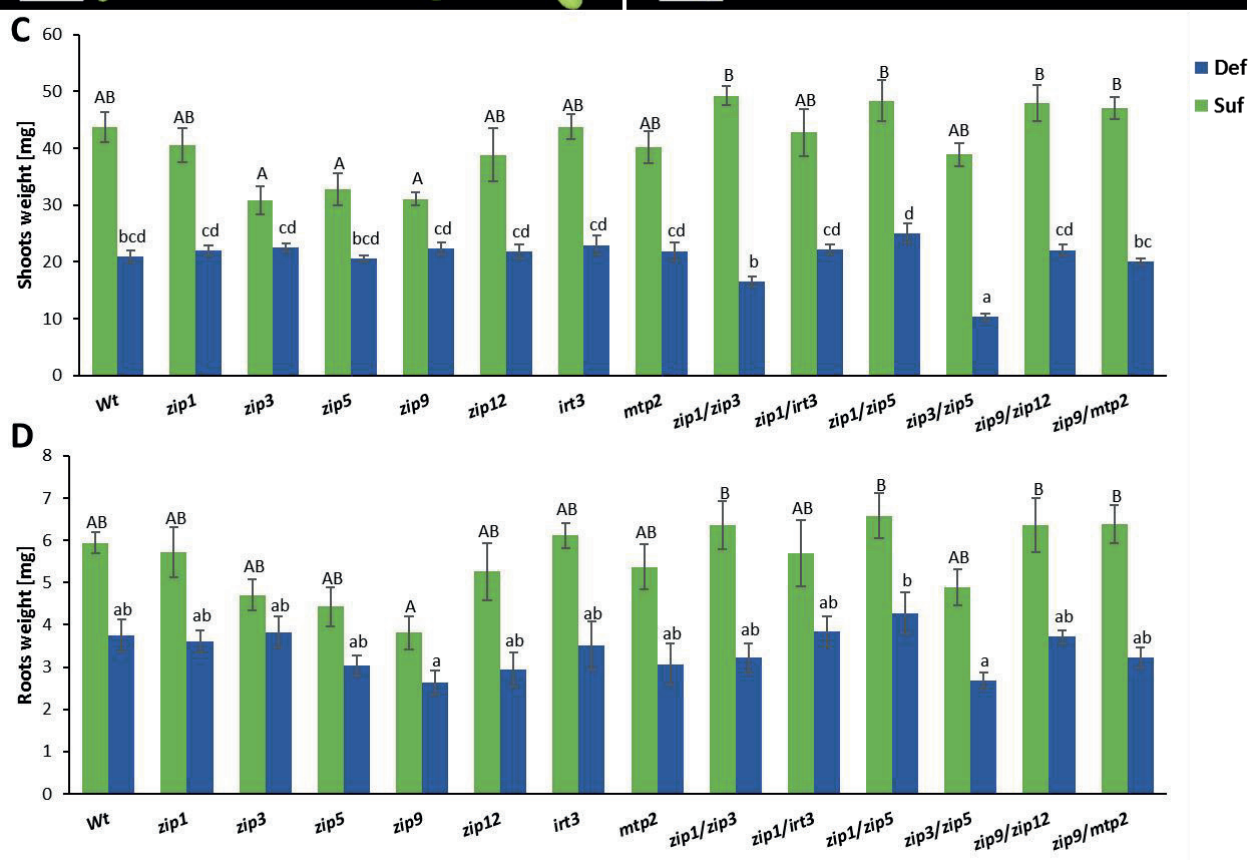
Figure 5. Response of single and double Zn transporter T-DNA insertion lines grown under Zn sufficiency and Zn deficiency. Two representative plants of wild-type and single and double Zn transporter T-DNA insertion lines grown hydroponically (A) 25 days under Zn sufficiency and (B) 10 days under Zn sufficiency and 15 days under Zn deficiency are shown. The scale bars indicate $2 \mathrm{~cm}$ in $A-B$. (C) Shoot and (D) root dry weight of wild-type and Zn transporter T-DNA insertion line plants grown hydroponically 25 days under Zn sufficiency (green bars) and 10 days under Zn sufficiency and 15 days under Zn deficiency (blue bars). Mean \pm SE, $n=10$ plants. Upper case letters (Zn sufficiency) and lower case letter (Zn deficiency) above the bars denote statistically different groups, when comparing among genotypes grown under each treatment, obtained with a Tukey post hoc test ( $\alpha=0.05)$, after a one-way ANOVA $(p<0.01)$.
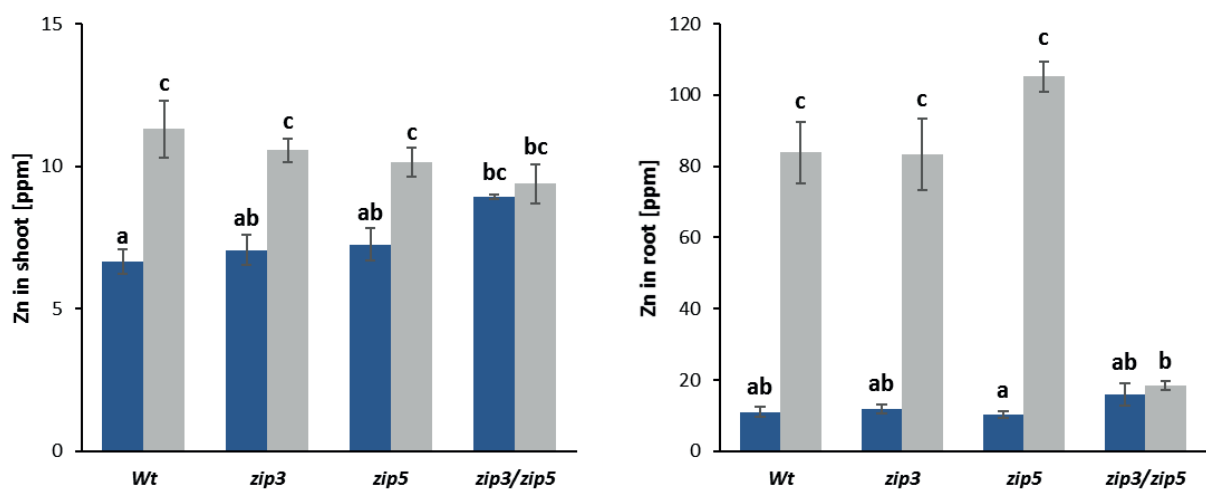

Figure 6. Zinc concentration of single and double, zip3 and zip5 T-DNA insertion lines. Zn concentration in (A) shoots and (B) roots measured in wild-type (Wt) and single and double zip 3 and zip5 T-DNA insertion lines grown in soil for 15 days and 30 days hydroponically under Zn deficiency (blue bars) plus 3 hours of Zn sufficiency before harvesting (grey bars) are shown. Mean $\pm S E, n=6$ plants. Letters above the bars denote statistically different groups, obtained with a Tukey post hoc test $(\alpha=0.05)$, after a two-way ANOVA ( $p<0.01$ genotype $x$ treatment interaction).

\section{Discussion}

\section{Loss-of-function of $\mathrm{Zn}$ transporters affects $\mathrm{Zn}$ distribution in Arabidopsis $\mathrm{Zn}$ roots}

When plants face nutrients deficiency, a strategy for survival is to transport the nutrients to metabolic active organs. In aerial parts, micronutrients like $\mathrm{Zn}$ can be remobilized from senescent leaves and stems by the phloem to growing organs (Maillard et al., 2015; DiazMendoza et al., 2016). But in roots, the half-hidden of the plant, we do not have a clear knowledge of this process. Therefore, we traced $\mathrm{Zn}$ distribution in whole roots of $\mathrm{Zn}$ deficient plants by using the Zimpyr-1 fluorescence dye (Woodroofe et al., 2004; Sinclair et al., 2007). 
In these roots, $\mathrm{Zn}$ concentrates mainly in growing zones such as main root tip, lateral root primordia, secondary root tips, and transition zone, meaning that during shortage, nutrients are still available for younger plant organs (Himelblau and Amasino, 2001; Avice and Etienne, 2014). In addition, root tips can be an easy access point to the root stele considering the lack of Casparian strip or suberin layer in this root zone. In the root differentiation zone, most of the $\mathrm{Zn}$ seems to be concentrated in the stele, which could subsequently be translocated from root to shoot. As we observed when comparing the stele Zinpyr-1 fluorescence among five-, six-, and seven- days-old Arabidopsis seedlings, the concentration of $\mathrm{Zn}$ in the stele of the older regions of the differentiation zone disappear when the plant suffer from $\mathrm{Zn}$ deficiency for a prolonged period of time. Therefore, the translocation of $\mathrm{Zn}$ to the shoot will be affected.

With the use of Zinpyr-1, we found different Zn deficiency distribution patterns through time, thus we decided to use the same approach to study the pattern of $\mathrm{Zn}$ distribution in $\mathrm{Zn}$ transporter mutants. The Zinpyr-1 fluorescence distribution pattern of five-day-old zip1, zip3, zip5, and in a lesser degree ysl3 mutants is similar to the pattern observed in five-day-old wild type. This indicates that $\mathrm{Zn}$ deficiency appears earlier in these mutants than in wild-type plants. In addition, the Zinpyr-1 fluorescence is less intense in the lateral root primordia of zip1, zip3 and zip5 than in the wild type. The most similar Zinpyr-1 fluorescence distribution pattern is between zip 3 and zip 5 mutants. In both mutants in the older region of the differentiation zone, the Zinpyr-1 fluorescence is almost absent in the stele, while present in cortex, epidermis, and pericycle. This particular pattern could be explained by a possible increase in expression of other $\mathrm{Zn}$ transporter like ZIP4, which is expressed in cortex and pericycle (Lin et al., 2016). These observations suggest that the plant machinery shouts down earlier the $\mathrm{Zn}$ allocation to the root stele in the mutants zip1, zip3, zip5 and ysl3 than in the wild type. From the five mutant lines analysed, the irt 3 mutant did not show clear differences in the Zinpyr-1 fluorescence distribution pattern when compared to wild type.

\section{Zn deficiency response cascade stats in roots}

One of the strategies to cope with fluctuating availability and concentrations of nutrients relies on changes in genes expression (Maillard et al., 2015). The transcriptome within cells and organisms is very flexible and constitutes a key factor to rapidly respond and adapt to changes in the environment. The dynamics of transcription responses allows for coordinated development and stress response by reshaping metabolic and physiological processes relevant for a given stress like nutrient deficiency (de Nadal et al., 2011). Arabidopsis plants were able 
to sense $\mathrm{Zn}$ deficiency in the medium and enhance the transcription of $\mathrm{Zn}$ transporters in roots after six hours of exposure to Zn deficiency. The first genes to show a significant induction are ZIP1 and ZIP4 after six hours, ZIP3, ZIP5 and IRT3 after twelve hours, and ZIP1, ZIP3, and IRT3 after thirteen hours. During the first hours of exposure to $\mathrm{Zn}$ deficiency, only genes of the ZIP family are significantly induced and only in the root. Due to the protein topology of the ZIP family, these protein transport metal ions from the apoplasm or organellar lumen to the symplast (Eide et al., 1996). Therefore, the first response to Zn deficiency of the plant seems to be to increase the uptake of $\mathrm{Zn}$ from the soil in the cell symplast. In the first day of exposure to Zn deficiency, the highest pick of induction was detected after twelve hours, when ZIP4, ZIP3, ZIP5, ZIP12, IRT3 in roots and ZIP12 in shoots showed above a three-fold induction. This induction could be a $\mathrm{Zn}$ deficiency solely or a combined response due to diurnal or circadian clock effect interaction (Grundy et al., 2015; Feeney et al., 2016) interaction. To avoid this combined effects from the twelfth hour of day one to day twelve, all samples were collected at the same time in the evening.

The second day at 8:00 in the evening, the gene expression of transporters in shoots and roots slightly dropped. This response could be the effect of a transient stabilization in the symplastic levels of Zn, due to the initial upregulation (Shalem et al., 2008; Yosef and Regev, 2011) of genes in charge to mobilize $\mathrm{Zn}$ into the symplast. Gene expression levels tend to return towards the original levels when cells are close to the unstressed state, even with continuous environmental stimuli (Yosef and Regev, 2011). We took the next sample at day four of exposure to $\mathrm{Zn}$ deficiency, when the levels of induction increase again in roots, showing a significant increase in the expression of ZPI1, ZIP4, and YSL3. The activity of YSL3 has been associated with the long distance transport of cations (Waters et al., 2006; Curie et al., 2009). Since day four, we also detected the significant increase in shoots of the expression of ZIPI, ZIP4, ZIP5, ZIP12, IRT3, HMA2, MTP2, and YSL3. This set of genes includes Zn symplast importers as the ZIP family (Eide, 2006) and Zn symplast exporters as HMA2 (Eren and Argüello, 2004) and MTP2 (Eide, 2006). This suggests that at day four, the plant starts to modify its $\mathrm{Zn}$ deficiency response cascade. The transcriptome fine tuning will determine the magnitude and duration of the response according to the degree of the environmental stimuli (Lopez-Maury et al., 2008). The highest induction for most of the genes in shoots and root was detected at day twelve. At this time point, ZIP1, ZIP3, ZIP5, ZIP9, ZIP11, ZIP12, IRT3, and MTP2 in shoots, and ZIP1, ZIP3, ZIP5, ZIP9, ZIP12, IRT3, HMA2 and MTP2 in roots are significantly induced by $\mathrm{Zn}$ deficiency. The only exceptions were HMA2 in shoots, ZIPII in 
roots, and YSL3 in shoots and roots, which showed a different pattern of expression form the rest of the genes tested. These three genes lack the ZDRE motif in their promoter sequences, which is present in the promoter sequences of ZIP1, ZIP3, ZIP4, ZIP5, ZIP9, ZIP12, and IRT3. This motif is the target sequence for binding bZIP19 and bZIP23 transcription factors that regulate the adaptation to $\mathrm{Zn}$ deficiency (Assunção et al., 2010). When the same transcription factors control a group of genes, these genes will share the same expression pattern (Yosef and Regev, 2011). In addition, MTP2 and ZIP12 showed the largest gene expression induction due to $\mathrm{Zn}$ deficiency. The expression of both genes was almost not detectable in normal $\mathrm{Zn}$ supply. However, MTP2 promoter only contains two palindromic sequences $80 \%$ similar to the ZDRE. So, its high level of induction by $\mathrm{Zn}$ deficiency could be regulated by transcription factors different from bZIP19 and bZIP23.

As mentioned before, another gene without a ZDRE domain in its promoter is $H M A 2$. This gene is known to be involved in $\mathrm{Zn}$ symplast efflux for root to shoot $\mathrm{Zn}$ translocation (Hussain et al., 2004; Sinclair et al., 2007). This gene was induced only above a three-fold change at day twelve in roots. HMA2 and HMA4 have a combined role in the $\mathrm{Zn}$ symplastic efflux and translocation to shoots (Hussain et al., 2004; Sinclair et al., 2007). However, HMA4 is not induced by $\mathrm{Zn}$ deficiency (van de Mortel et al., 2006). Therefore, during Zn deficiency, probably the root to shoot translocation is not prioritized over the $\mathrm{Zn}$ root uptake.

\section{Zn transporters express in different cell layers}

Water and nutrients move radially through the root concentric cell layers to enter from the soil solution to the xylem where they will be translocated to the shoot. The concentric cell layers from the outer to the most inner layer are: epidermis, cortex, endodermis, and stele which consist of pericycle, xylem, phloem, companion cells and procambium (Barberon and Geldner, 2014). The tested $\mathrm{Zn}$ transporters are expressed in different combinations across the root cell layers. A summary of the cell layer where each transporter is expressed is presented in figure 7. ZIP1 expresses in the endodermis and root stele. The ZIP1 early expression and its putative location in the tonoplast (Milner et al., 2013) indicates that the Zn deficiency response initially promotes the increase of the $\mathrm{Zn}$ symplastic concentration by using the vacuole as a nutrient storage compartment, which becomes a side-branch of the symplastic pathway (Tester and Leigh, 2001). 


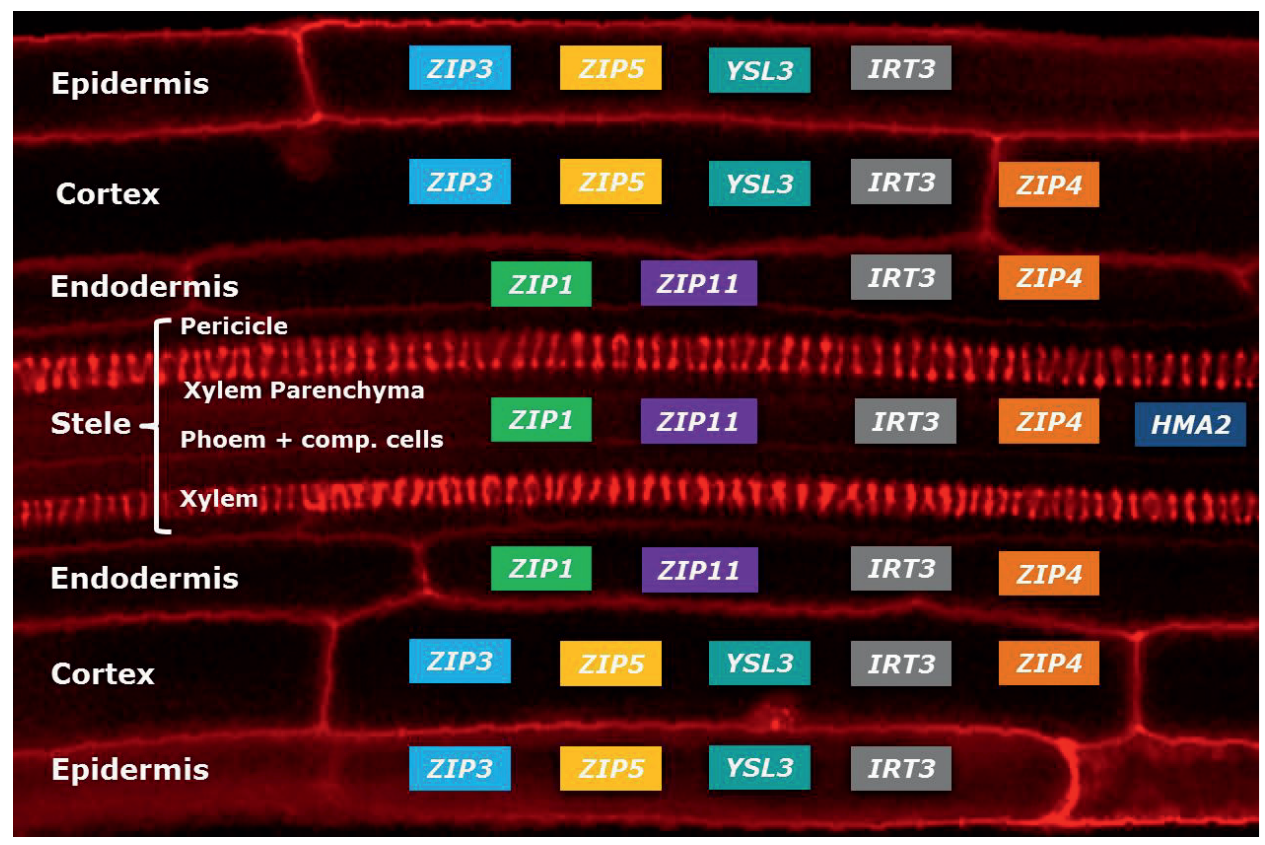

Figure 7. Summary of the cell layer where each Zn transporter is expressed. The scheme is based in a confocal laser-scanning microscope image of Arabidopsis root. The red fluorescent signal indicates mainly cell walls stained with propidium iodide. The cell layers are label as epidermis, cortex, endodermis and stele, which includes pericycle, xylem, xylem parenchyma, phloem and companion cells. Transporters are represented by different coloured rectangles.

The symplastic pathway starts with the $\mathrm{Zn}$ uptake into the symplast. The expression of ZIP3 and ZIP5, which are located in the root outer layers (epidermis and cortex), constitutes the first step for the Zn symplastic pathway. Epidermis and cortex are functionally similar in terms of nutrients absorption (Barberon and Geldner, 2014). The expression of ZIP3 and ZIP5 in epidermis and cortex assures the symplastic pathway even in older zones of the root. In these zones, the endodermis becomes a barrier for the apoplastic pathway due to the Casparian strip (Geldner, 2013). The Zn transporter IRT3, promotes uptake into the symplast (Lin et al., 2009), in all the root from the epidermis to the stele. The expression of ZIP4 is mainly in cortex, endodermis, and pericycle, which likely facilitates the Zn entry to the stele (Lin et al., 2016). The role of $\mathrm{Zn}$ efflux into the stele apoplast is mainly attributed to the HMA2 and HMA4 (Hussain et al., 2004; Sinclair et al., 2007). However, only HMA2 shows to be induced by $\mathrm{Zn}$ deficiency (van de Mortel et al., 2006). We observed HMA2 expression in an inner-layer of the stele in xylem parenchyma cells. This result differs slightly with Sinclair, 2007, where a HMA2p-HMA2-GFP showed expression in pericycle cells. Nonetheless, in both cases, the 
expression is detected in stele cells, which can favour the availability of $\mathrm{Zn}$ in the stele apoplast. The expression location of ZIP11 was particularly interesting, due to its expression in xylem parenchyma cells in the young differentiation zone of the roots, and in endodermal cells in the older differentiation zone of the roots. The cell layer where ZIP9, ZIP12 and MTP2 express could not be defined, because they only showed expression in the transition zone, however, lateral roots were not analysed.

\section{The function of $\mathrm{Zn}$ transporters is redundant}

Zn deficiency significantly reduces the biomass production of plants (Campos et al., 2017) either wild-type or $\mathrm{Zn}$ transporter mutants. Under normal $\mathrm{Zn}$ supply, the biomass of shoots and roots of the single mutants zip3, zip5, and zip9 was lower than the biomass of wild-type plants, and the double mutants zip1/zip3, zip1/zip5, zip9/zip12 and zip9/mtp2 was higher than the biomass of wild-type plants, however, the difference is not statistically different. Under $\mathrm{Zn}$ deficiency, almost all mutant genotypes have similar weight in shoot and root than the wild type, the only exception is zip3/zip5. The double mutant zip3/zip5, is the most sensitive genotype to $\mathrm{Zn}$ deficiency, with yellow curly leaves and a significant decrease in shoot biomass compared to wild type. The double mutant zipl/zip3, also shows to be more sensitive to $\mathrm{Zn}$ deficiency than the wild type, but this difference is not statistically different. The lack of reports showing the effect of the loss of function of the $\mathrm{Zn}$ transporters here analysed and the small or null effect of single $\mathrm{Zn}$ transporters mutants strongly suggest redundant functions among $\mathrm{Zn}$ transporters, especially in the case of zip3 and zip5. Both genes are phylogenetically closely related (Mäser et al., 2001), are expressed in the same cell layers in the root, the $\mathrm{Zn}$ distribution is similar in their loss-of-function mutants, and their double loss-of-function mutant is highly sensitive to $\mathrm{Zn}$ deficiency.

Considering that zip3/zip5 was the only mutant statistically different from the wild type in $\mathrm{Zn}$ deficiency, we analyzed its $\mathrm{Zn}$ concentration and uptake capacity. The wild-type, zip 3 and zip5 single mutants have a similar $\mathrm{Zn}$ concentration among them in shoots and roots when plants grown under Zn deficiency conditions. Surprisingly, the double mutant zip3/zip5 had a significantly higher concentration of $\mathrm{Zn}$ in shoots than wild type. After a three hours $\mathrm{Zn}$ supply wild-type, zip3 and zip5 single mutants significantly increased their $\mathrm{Zn}$ concentration in shoot and roots, while zip3/zip5 shows no increase in $\mathrm{Zn}$ concentration. This suggests that ZIP3 and ZIP5 are involved in Zn uptake in the roots. 


\section{Conclusions}

All Zn transporters studied here are induced by Zn deficiency either in shoot or in roots, after hours and/or after days of exposure to $\mathrm{Zn}$ deficiency. The $\mathrm{Zn}$ deficiency response initiates in the roots and later is induced in shoots. In roots, each $\mathrm{Zn}$ transporter gene is expressed in one, or more, specific root cell layer(s), from epidermis till stele. This location may differ, depending on the root region. These transporters showed partial functional redundancy among them because the single or double loss-of-function of $\mathrm{Zn}$ transporters do not cause strong sensitivity to $\mathrm{Zn}$ deficiency, except for the double mutant zip3/zip5. The ZIP3 and ZIP5 genes seem to be highly redundant and to be involved in $\mathrm{Zn}$ uptake from roots.

\section{Acknowledgements}

We thank Prof. Richard Immink, Alice Pajoro, and Tjitske Ricksen (Wageningen University) for facilitating the use of the confocal microscope and providing the technical support. We thank Prof. Henk Schat (VU Amsterdam) for his help in the Zn uptake assay. This work was supported by the Ecuadorian government through SENESCYT (Secretaría de Educación Superior, Ciencia, Tecnología e Innovación) and Universidad de las Fuerzas Armadas-ESPE. 


\section{Supplemental information}

Table S1. T-DNA insertion lines of Zn transporter genes with their respective LB (left border) and RP (right border) primers to genotype for homozygote T-DNA insertion plants.

\begin{tabular}{|c|c|c|c|c|}
\hline Symbol & Locus identifier & T-DNA line & Primer & Sequence $\left(5^{\prime}-3\right)$ \\
\hline \multirow{2}{*}{$Z I P 1$} & \multirow{2}{*}{ At3g12750 } & \multirow{2}{*}{ SALK_023634C } & LB & CTTGAGGACACTACAGCTGGG \\
\hline & & & $\mathrm{RB}$ & GAATGCGGATTATTAGCTGG \\
\hline \multirow{2}{*}{ ZIP3 } & \multirow{2}{*}{ At2g32270 } & \multirow{2}{*}{ SAIL_35_B08 } & LB & GGCTCGTTACTTTCCTTGGAC \\
\hline & & & $\mathrm{RB}$ & CCATTATTTGGAATGCAATCG \\
\hline \multirow{2}{*}{ ZIP5 } & \multirow{2}{*}{ At1g05300 } & \multirow{2}{*}{ SALK_009007C } & LB & AGGGACAAAAACAAATACGGG \\
\hline & & & $\mathrm{RB}$ & AGGCAAATGAAAATTCAGTGAC \\
\hline \multirow{2}{*}{ ZIP9 } & \multirow{2}{*}{ At4g33020 } & \multirow{2}{*}{ SALK_074682C } & LB & ACACCGATCGGAGTAGTGATG \\
\hline & & & $\mathrm{RB}$ & TTTCGGTTATGTCTGACCCAG \\
\hline \multirow{2}{*}{$Z I P 10$} & \multirow{2}{*}{ Atlg31260 } & \multirow{2}{*}{ SALK_104586C } & LB & TTTGCTTTTATTTGAGTGACAACC \\
\hline & & & $\mathrm{RB}$ & TCTGCATTCCATAAATGTCAAG \\
\hline \multirow{2}{*}{ ZIP11 } & \multirow{2}{*}{ At1g55910 } & \multirow{2}{*}{ SALK_120099C } & LB & TTGGTTGATCTTCCTGTTTGG \\
\hline & & & $\mathrm{RB}$ & AGGATTTGGATTTGAGATCGG \\
\hline \multirow{2}{*}{ ZIP12 } & \multirow{2}{*}{ At5g62160 } & \multirow{2}{*}{ SALK_137184 } & LB & AATGTCCTTGTGAGGCATGAG \\
\hline & & & $\mathrm{RB}$ & GATTTCTCCCTGGTCGAAGAG \\
\hline \multirow{2}{*}{ IRT3 } & \multirow{2}{*}{ Atlg60960 } & \multirow{2}{*}{ WiscDsLox429D04 } & LB & TGATGTGTGTTTTGCTCTTCG \\
\hline & & & $\mathrm{RB}$ & AGCAATGGATAAGAAATTGCG \\
\hline \multirow{2}{*}{ MTP2 } & \multirow{2}{*}{ At3g61940 } & \multirow{2}{*}{ SALK_003649C } & LB & GCTGCAGATGGATTCAAGAAG \\
\hline & & & $\mathrm{RB}$ & ATGAGAGCATACGAGAAAGCG \\
\hline \multirow{2}{*}{ YSL3 } & \multirow{2}{*}{ At5g 53550} & \multirow{2}{*}{ SALK_045218C } & LB & GCCTTTAGGAGTGTGGAAACC \\
\hline & & & $\mathrm{RB}$ & TTTTTCCTCTCGTCATTTTCC \\
\hline \multirow{2}{*}{$H M A 2$} & \multirow{2}{*}{ At4g30110 } & \multirow{2}{*}{ SALK_034393 } & LB & GGAGAGTGACTCCCTAAAGCC \\
\hline & & & $\mathrm{RB}$ & TAAACAAGAATGGCGTCGAAG \\
\hline
\end{tabular}

Table S2. Sequences of primers used for gene expression quantification by qRT-PCR

\begin{tabular}{cll}
\hline Symbol & Primer Forward (5'-3) & Primer Reverse (5'-3) \\
\hline ZIP1 & TCTCCCTGGCGGATATGAAGTC & TCCACCATTATTGCCTCTTTGCTC \\
ZIP3 & CTCCTTCTCATCGCCGTCGT & CGAGCTCCGGCTTTGTTTTC \\
ZIP4 & GGCTGCATCTCTCAGGCACA & GGCCACTGCAGTTCCAATCC \\
ZIP5 & CGTCGCTTAAACCGGAGACG & CATAAACCCCGTTGCGAGGA \\
ZIP9 & ACTTGTGTACATGGCGCTTG & TGCAAGAGCAGACATCATCC \\
ZIPI1 & TTGGTACACAATTCGCCGGA & AAGCAAATGGGTAAGCCGGA \\
ZIPI2 & CCATCTTAATCGCCGGAGTA & TCTTCTCCAAGGCACGAACT \\
IRT3 & GATTCTCGCCACGGGTTTTG & GCAAAGAATCCGGGAAAGG \\
HMA2 & TCCGTCAAGAACCGTCATCGTC & AACGATTTGGAACTGCGAGAGG \\
MTP2 & CGAGCTGCTTCAATGCGAAA & AGCACCAACATCAGTGAGCA \\
YSL3 & GGTGGTACAGAGTGCGGTTT & TGATTCCCCAAGACAGAACC \\
SAND & GTTGGGTCACACCAGATTTTG & GCTCCTTGCAAGAACACTTCA \\
\hline
\end{tabular}


Table S3. Sequences of primers used to generate the destination vector. Underlined oligoes represent the primers linkers.

\begin{tabular}{ll}
\hline Primers description & Sequence (5'- $\mathbf{3}^{\prime}$ ) \\
\hline Forward for NLS & ATTCACATTCTTGCCCGCCT \\
Reverse for NLS plus a sYFP2 linker & GCCCTTGCTCACCATAGGTTGAGAAGATGGCTCTATTTTC \\
Forward for sYFP2 plus a NLS linker & $\underline{\text { CCATCTTCTCAACCTATGGTGAGCAAGGGCGAGGAGCTGT }}$ \\
Reverse for sYFP2 & CCTTCAACGTTGCGGTTCTG \\
Forward for the DJ fragment & TCTTGCCCGCCTGATGAATG \\
Reverse for DJ fragment plus a NRUI linker & TTCTCGCGATCTAGTAACATAGATGACAC \\
\hline
\end{tabular}

Table S4. Sequences of primers used to clone gene promoters. Forward primers include the attB1 linker (GGGGACAAGTTTGTACAAAAAAGCAGGCTTA) and reverse primers include the attB2 linker (GGGGACCACTTTGTACAAGAAAGCTGGGTA)

\begin{tabular}{lll}
\hline \multicolumn{1}{c}{ Promoter } & \multicolumn{1}{c}{ Primer Forward } & \multicolumn{1}{c}{ Primer Reverse } \\
\hline$Z I P 1$ & CAGTTATGCAAATACTCCGG & TGAGTTTAAGATATTTATGTTCTTGTT \\
$Z I P 3$ & TATCCAGAAAGATGATGTGTATACA & AATCTCTATCTTATTTTAAAATTAGGG \\
$Z I P 5$ & TATGTTTTTATTTTGAGGCACAAATT & CTTATCGATTAGGGTTTGAATTTGA \\
$Z I P 9$ & CAGATATGCCCAAAGCATATTCTT & TAGCTGCGAACTTGAGGGTAA \\
$Z I P 10$ & TTGTCTTCTTTTTCCCGCTTC & CTTTCTATTTGTTTCTTGTGGAGTTTT \\
$Z I P 11$ & GAACTTTGAGAAATTATTGGTTGAAAC & TTTGAATGTTCAGTGGGTTTGT \\
$Z I P 12$ & GAGTTGTGTACCGTGAGTTGATTATT & TCGTTTACTTTTGACAAAAGTTAGG \\
$I R T 3$ & ACTCTTTTTCATGTTCCATACACAATT & TTGGGGTCTAAGATGTCCTCG \\
HMA2 & CATCTCAAAAGGTAAACTTAAAGACAA & CTGCAGCAAAAAAGATTGTAACTTT \\
$M T P 2$ & AACTATTCCAAATGAGGAATCACAC & TCTTGTTTAAGGATTCTGCA \\
YSL3 & CGTCCGAATATAATAGTCCAATTCTAC & TTTTTTCCAAGAACAGAACAAAAAA \\
\hline
\end{tabular}




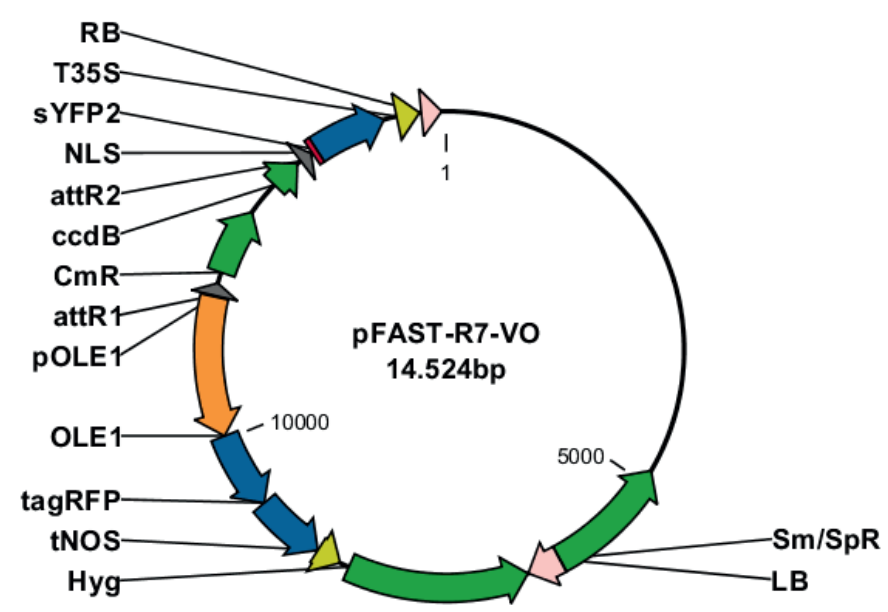

Figure S1. Gateway destination vector was generated to clone the promoter sequences. FAST-R07 (Shimada et al., 2010) modified vector.

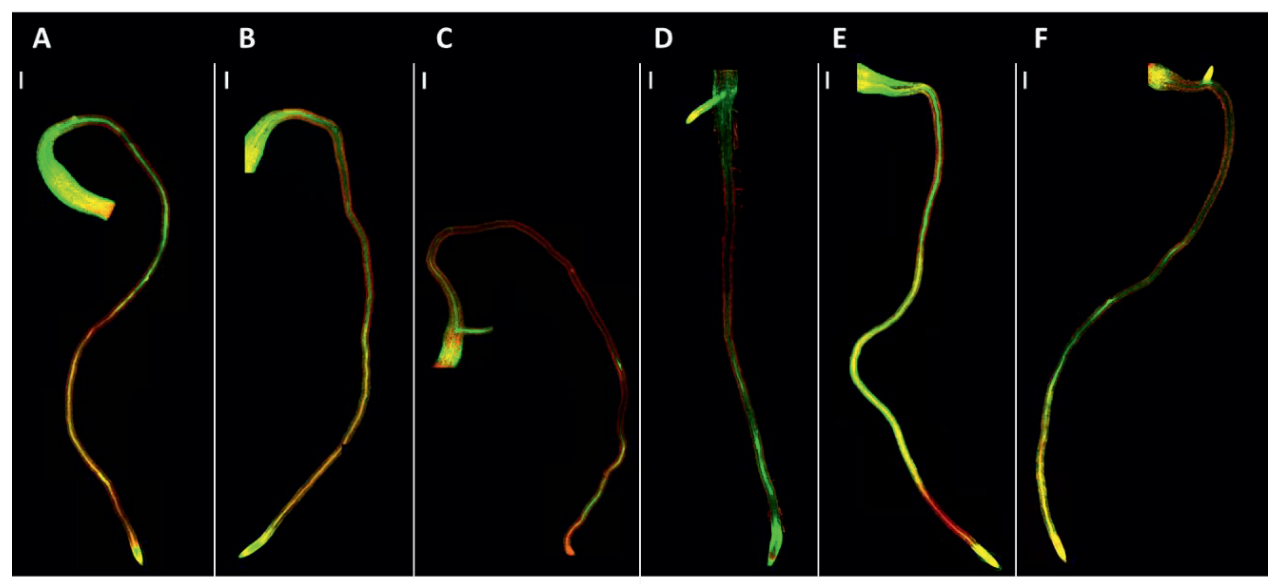

Figure S2. Confocal laser scanning microscope images of Zn distribution in Arabidopsis roots of wildtype and T-DNA insertion lines. (A)Zn deficient Arabidopsis roots of five-day-old Col-0 wild-type, zip1 (B), (C) zip3, (D) zip5, (E) irt3 and (F) ysl3 plants incubated with Zinpyr-1 to detect Zn. Zinpyr-1 complexed to Zn fluoresces green, while propidium iodide bound to cell wall or DNA fluoresces red. The scale bars indicate $200 \mu \mathrm{m}$. 
Shoot

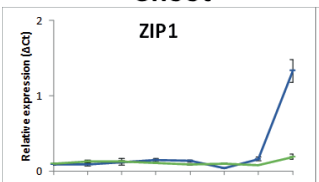

$0.25 \mathrm{~h}$ in $3 \mathrm{~h}$ 6h $12 \mathrm{~h}$ 36h $4 \mathrm{~d} \quad 12 \mathrm{~d}$

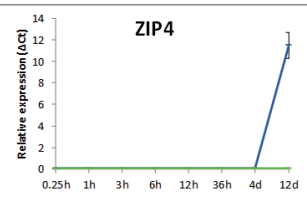

$\begin{array}{llllllll}0.25 \mathrm{~h} & \text { 1h } & 3 \mathrm{~h} & 6 \mathrm{~h} & 12 \mathrm{~h} & 36 \mathrm{~h} & 4 \mathrm{~d} & 12 \mathrm{~d}\end{array}$

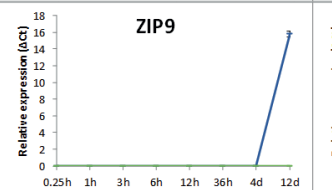

$0.25 \mathrm{~h} \quad$ th $\quad 3 \mathrm{~h} \quad 6 \mathrm{~h} \quad 12 \mathrm{~h} \quad 36 \mathrm{~h} \quad 4 \mathrm{~d} \quad 12 \mathrm{~d}$

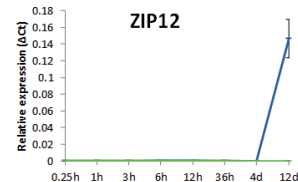

$0.25 \mathrm{~h}$ in $3 \mathrm{~h}$ 6h $12 \mathrm{~h}$ 36h ad $12 \mathrm{~d}$
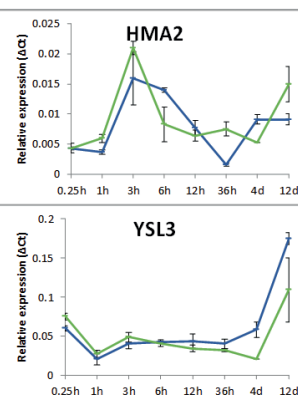

Root

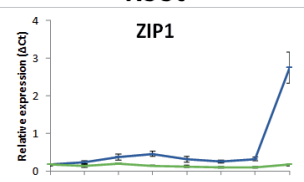

$0.25 \mathrm{~h}$ in $3 \mathrm{~h}$ 6h $12 \mathrm{~h} \quad 36 \mathrm{~h} \quad 4 \mathrm{~d} \quad 120$
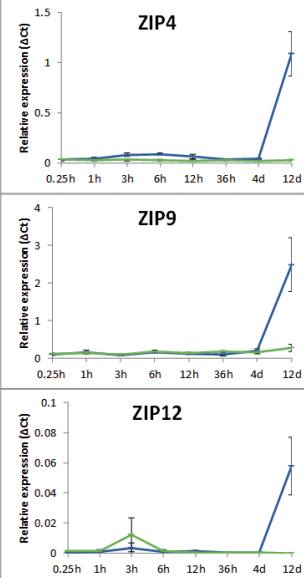

$0.25 \mathrm{~h}$ in 3 h 5 oh $12 \mathrm{~h}$ 36h 4 d $12 \mathrm{~d}$

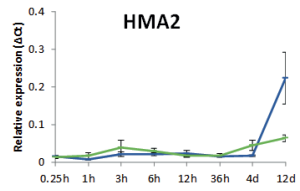

$0.5 \quad$ YSL3

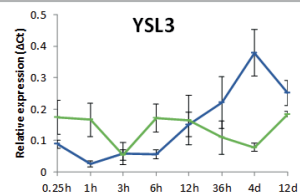

Shoot
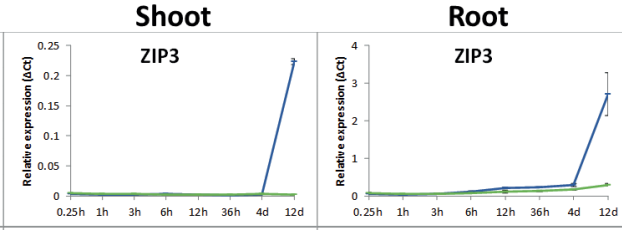

ZIP5

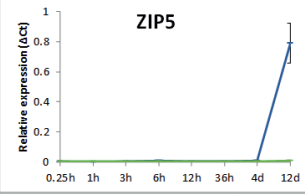

ZIP5

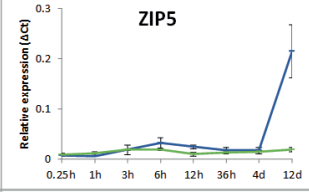

ZIP11
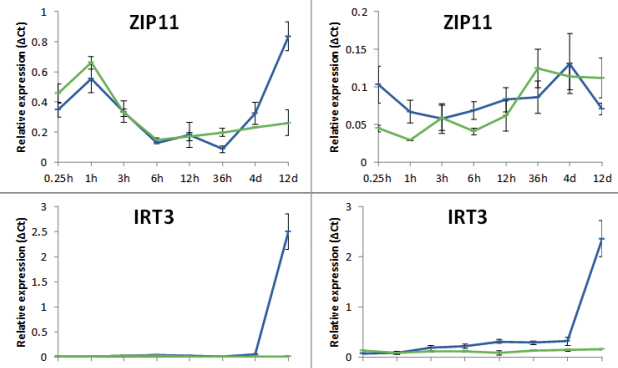

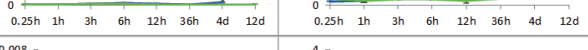

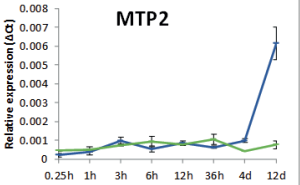

MTP2

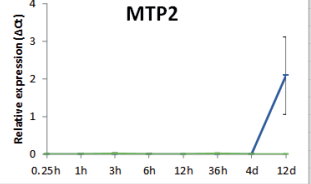

- Sufficiency

-Deficiency

Figure S3. Gene expression in shoots and roots of Arabidopsis in response to Zn deficiency. Gene expression relative to At5g25760 and AT2G28390 in shoots and roots of plants grown for ten days in fully supplied medium and then in either Zn deficiency (blue lines) or Zn sufficiency (green lines) during 12 days. Samples were taken at several time points form 0.25 hours (h) to 12 days (d) after applying the treatment. Mean $\pm S E, n=3$ of 3 pooled plants. 


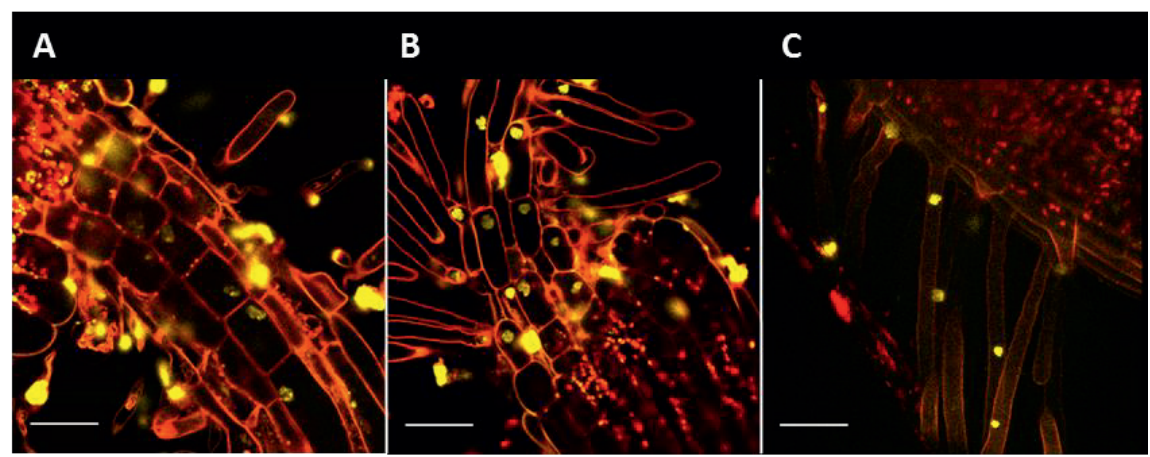

Figure S4. Confocal laser scanning microscope images of Arabidopsis roots expressing nuclearlocalized YFP driven by promoter regions of three Zn transporters. The SYFP2 fluorescent signal (yellow) can be seen in all nuclei of cells expressing the NLS-SYFP2 marker gene. The red fluorescent signal indicates mainly cell walls stained with propidium iodide. Arabidopsis root transition zone expressing NLS-SYFP2 driven by the promoter regions of (A) ZIP9, (B) ZIP12, and (C) MTP2. Yellow spots bigger than nuclei, in the transition zone, correspond to the autofluorescence of seed fragments. The scale bars indicate $50 \mu \mathrm{m}$ in $\mathrm{B}-\mathrm{H}$. 




\section{Chapter 3}

\section{Disruption of N-terminal-acetylation disturbs mineral homeostasis in Arabidopsis thaliana}

Valeria Ochoa Tufiño ${ }^{1,3}$, Ana Carolina Campos ${ }^{1,4}$, Joost van den Heuvel $^{1}$, Ana G. L. Assunção $^{1,5}$, Eric Linster ${ }^{2}$, Markus Wirtz ${ }^{2}$ and Mark G.M. Aarts ${ }^{1}$

1) Laboratory of Genetics, Wageningen University, Wageningen, The Netherlands

2) Centre for Organismal Studies, University of Heidelberg, Heidelberg, Germany

3) Current affiliation: Departamento de Ciencias de la Vida, Universidad de las Fuerzas Armadas ESPE, Sangolquí, Ecuador

4) Current affiliation: Dümmen Orange, Leiderdorp, The Netherlands

5) Current affiliation: Department of Plant and Environmental Science, University of Copenhagen, Copenhagen, Denmark

In preparation for submission 


\begin{abstract}
The functional diversity of the plant proteome is large, even larger than the diversity of the plant transcriptome, this is as a result of several types of protein modifications that alter protein activity, lifespan, localization and protein-protein interactions. N-terminal-acetylation (N-tacetylation) is a protein modification that is widespread among eukaryotes, carried out by Nalpha-terminal-acetyltransferase complexes (Nats). After a mutation mapping approach in Arabidopsis thaliana (Arabidopsis) we identified a mutation in the N-ALPHA-TERMINAL ACETYLTRANSFERASE 25 (NAA25) gene, which encodes the non-catalytic subunit of NatB, to be responsible for aberrant regulation of the $\mathrm{Zn}$ deficiency response. We tested several additional mutants of $N A A 25$, all which displayed pleiotropic effects affecting plant morphology, sensitivity to Fe deficiency, and to a lesser degree the deregulation of $\mathrm{Zn}$ deficiency response. To further understand the processes involved, we examined the transcriptome of a naa25 mutant under control, $\mathrm{Zn}$ or Fe deficiency medium. The mutant showed differential expression of genes involved in plant defence response under all tested conditions. Several $\mathrm{Zn}$ deficiency responsive genes showed a slightly but consistently high in expression in naa25, under fully supplemented medium, compared to wild-type plants. Upon Fe deficiency, genes involved in Fe distribution and sequestration are higher expressed in the mutant than the wild type. In addition, several genes encoding components of Photosystem I (PSI) and PSII are differentially expressed in naa25. Although Zn and Fe deficiency responses are affected in the naa 25 mutant, these appear to be pleiotropic effects of a general de-regulation of protein N-t-acetylation, which affects several other processes involved in plant growth and development.
\end{abstract}




\section{Introduction}

Proteins can undergo different kinds of co- and/or post-translation modifications (Karve and Cheema, 2011). One of these modifications is $\mathrm{N}$-t-acetylation, which generally occurs during translation. N-t-acetylation is very common in most eukaryotes. Around $80 \%$ of mammalian and plant proteins are N-t-acetylated (Pesaresi et al., 2003; Linster et al., 2015; Aksnes et al., 2016). N-t-acetylation can affect protein stability, subcellular location, interaction with membranes, protein-protein interactions, protein folding, and aggregation. It involves the transfer of acetyl groups from acetyl-CoA to the $\alpha$-amino group of the protein N-terminalresidue. This process is carried out by ribosome-associated $\mathrm{N}$-alpha-terminal-acetyltransferase (NAA) enzymes (Polevoda and Sherman, 2000; Gibbs, 2015; Aksnes et al., 2016). The NAA proteins form complexes, named as Nats, with catalytic and non-catalytic auxiliary subunits (Polevoda and Sherman, 2003). A total of seven Nats (NatA-NatG) are described in eukaryotes. The most common are the NatA, NatB, and NatC, which are responsible for the majority of Nt-acetylation events (Linster et al., 2015).

Little information is available about NatA, NatB, and NatC in plants. In Arabidopsis the disruption of $N A A 30$, which encodes for the NatC catalytic subunit, decreases the accumulation of mature proteins in chloroplasts, affecting photosynthesis efficiency (Pesaresi et al., 2003). NatA impairment in Arabidopsis, due to the disruption of either NAA10 or NAA15, causes embryo lethality. These genes encode for the catalytic and auxiliary subunit of NatA respectively. Downregulation mutants of NAA10 or NAA15 show a decreased sulphate assimilation and increased drought tolerance (Linster et al., 2015). Downregulation of NAA10 or NAA15 also increases the stability of the plant immune receptor SUPPRESSOR OF NPR11, CONSTITUTIVE 1 (SNC1). On the other hand, the stability of SNC1 decreases due to mutations in NAA20 or NAA25, which encode for the catalytic and auxiliary subunit of NatB (Xu et al., 2015). The phenotypes of naa10, 15, 20, 25 and 30 single mutants (Linster et al., 2015 ) suggest a major role of $\mathrm{N}$-t-acetylation in plant growth and adaptation to environmental changes (Gibbs, 2015).

Plants are continuously challenged to biotic and abiotic stresses that reduce plant fitness, crop yield, and food quality. One of these stresses is micronutrient deficiency, that is a shortage of Fe, $\mathrm{Zn}, \mathrm{Mn}, \mathrm{Cu}, \mathrm{Mo}, \mathrm{B}, \mathrm{Co}$, and/or Se. Micronutrient deficiencies often lead to stunted growth, poor development, small and twisted leaves, chlorosis or necrotic spots. $\mathrm{Zn}$ and $\mathrm{Fe}$ 
micronutrient deficiencies are widespread and occur at many sites worldwide, often associated with calcareous or (slightly) alkaline soils, in which the bioavailability of divalent cations is generally low (Alloway, 2009). Plant responses to mineral deficiencies are often specific for each mineral and involve several physiological adaptations, following the sensing and signalling of the deficiency.

The Fe deficiency response, involving the expression of several genes contributing to $\mathrm{Fe}$ acquisition and distribution, is regulated by several transcription factors, such as the FEDEFICIENCY-INDUCED TRANSCRIPTION FACTOR 1 (FIT1) and basic helix-loop-helix proteins bHLH38, 39, 100, 101, 104, 115 and POPEYE (PYE) (Colangelo and Guerinot, 2004; Wu et al., 2012; Wang et al., 2013; Liang et al., 2017). Compared to the control of Fe deficiency, less is known about the regulation of the $\mathrm{Zn}$ deficiency response, even though both elements share an intricate cross-homeostasis system (Shanmugam et al., 2011; Pineau et al., 2012; Briat et al., 2015). So far, the only identified regulators of the $\mathrm{Zn}$ deficiency response are the BASICREGION LEUCINE ZIPPER 19 (bZIP19) and bZIP23 proteins. Both transcription factors are expressed at low levels in plants and partly redundant, but without their function, plants are not able to induce the expression of several genes essential for a proper $\mathrm{Zn}$ deficiency response in mobilizing and acquiring more Zn from the soil (Assunção et al., 2010; Assunção et al., 2013).

We identified an naa25 mutant in a screen for mutants displaying an aberrant $\mathrm{Zn}$ deficiency response. The naa25 mutation promotes a mild $\mathrm{Zn}$ deficiency response under sufficient $\mathrm{Zn}$ conditions, but also a strong sensitivity to Fe deficiency. NAA25 encodes the auxiliary subunit of the NatB in Arabidopsis. We examined additional mutant phenotypes, including naa25 mutant transcriptome, and analysed the involvement of the NAA25 gene in the response to $\mathrm{Fe}$ and $\mathrm{Zn}$ deficiency to further understand the pleiotropic phenotype of the naa 25 mutant.

\section{Materials and Methods}

\section{Plant material and growth conditions}

T-DNA insertion lines were ordered from the Nottingham Arabidopsis Stock Centre (NASC; www.arabidopsis.info/BasicForm), except for naa20 (Salk-027687), which was provided by Markus Wirtz. Arabidopsis accession Columbia (Col-0) was used for, and depending on the background of T-DNA insertion lines either Col-0 or Col-4 were used as a wild-type control. Prior to germination, seeds were stratified for three days at $4{ }^{\circ} \mathrm{C}$ in the dark. The plants used for 
genetic analysis and transformation were grown in peat-based fertilized potting mixture or rockwool, in a greenhouse set at $16 / 8 \mathrm{~h}$ light/dark cycle at $20 / 18^{\circ} \mathrm{C}$ day/night temperatures and $70 \%$ humidity. For agar-plate-based phenotypic screens, plants were grown on agar plates in a climate-controlled growth chamber set at a $16 / 8 \mathrm{~h}$ light/dark cycle at $22 / 20^{\circ} \mathrm{C}$ day/night temperatures and 50\% humidity. Seeds were surface-sterilized using vapor-phase seed sterilization (Clough and Bent, 1998) and sown on 12-cm square petri dishes containing 1\% agar-solidified half-strength MS medium ( $\mathrm{pH}$ 5.8). The control medium contained $15 \mu \mathrm{M}$ $\mathrm{ZnSO}_{4}, 50 \mu \mathrm{M} \mathrm{Fe}(\mathrm{Na})_{2} \mathrm{EDTA}_{2}$ and $0.05 \mu \mathrm{M} \mathrm{CuSO}_{4}$. The media used for different mineral treatments contained the same concentrations except for: no added $\mathrm{Zn}$ in the $\mathrm{Zn}$ deficiency treatment, $150 \mu \mathrm{M} \mathrm{ZnSO}$ in the $\mathrm{Zn}$ excess treatment, $2.5 \mu \mathrm{M} \mathrm{Fe}(\mathrm{Na})_{2} \mathrm{EDTA}_{2}$ in the Fe deficiency treatment and $40 \mu \mathrm{M} \mathrm{CuSO}_{4}$ for the $\mathrm{Cu}$ excess treatment. Plant grown in agar plates were collected after three weeks of treatment and dried for three days at $60{ }^{\circ} \mathrm{C}$ to determine dry weights.

Plants were also grown hydroponically, in a climate-controlled growth chamber set at $70 \%$ humidity, a $12 / 12 \mathrm{~h}$ light/dark cycle, and $20 / 15^{\circ} \mathrm{C}$ day/night temperatures. Vapor-phase sterilized seeds were sown on $0.55 \%$ agar-filled tubes of which the bottom was cut-off, and grown on a modified half-strength Hoagland's nutrient solution (Schat et al., 1996). The first two weeks plants grew in a control medium ( $2 \mu \mathrm{M} \mathrm{ZnSO}_{4}$ and $\left.20 \mu \mathrm{M} \mathrm{Fe}(\mathrm{Na})_{2} \mathrm{EDTA}_{2}\right)$, and thereafter treatments were applied or plants were maintained to grow in the control medium. The treatments contained no added Zn for Zn deficiency or no added Fe for Fe deficiency.

\section{Generation of mutagenized population and screening}

Seeds of an Arabidopsis transgenic line (Col-0 background), stably transformed with a pZIP4::GUS construct (Lin et al., 2016) and containing multiple copies of this construct at one locus was used for $\gamma$-ray-mutagenesis. For this, seed batches of $65 \mathrm{mg}$ each were suspended in a solution of $10 \mathrm{mM} \mathrm{KNO}_{3}$ and $0.2 \%$ agar and exposed to a dose of 300 Grey $\gamma$-rays at Isotron (Ede, The Netherlands). One hundred pools of $\sim 100$ M1 seeds were sown in 100 trays with peat-based fertilized potting mixture in the temperature-controlled greenhouse. From each tray, a pool of M2 seeds was harvested and $\sim 400$ seeds per pool ( $\sim 40,000$ seeds) were screened for GUS expression. Seeds were sown on $15-\mathrm{cm}$ diameter round petri dishes containing $0.6 \%$ agarsolidified half-strength MS medium plus $1 \%$ sucrose. No $\mathrm{Zn}$ was added for $\mathrm{Zn}$ deficiency or 30 $\mu \mathrm{M} \mathrm{ZnSO}_{4}$ was added for $\mathrm{Zn}$ sufficiency. A non-destructive GUS-assay was used, which involved the application of $3 \mathrm{ml}$ of reagent mix on the surface of every $15-\mathrm{cm}$ diameter round 
petri dish. The reagent mix contained $2 \mathrm{mM}$ X-Gluc (5-Bromo 4-Chloro-3-Indolyl $\beta$-Dglucuronic acid cycloheximide salt) in $50 \mathrm{mM} \mathrm{NaH}_{2} \mathrm{PO}_{4}$ buffer $(\mathrm{pH}=7.2)$ and $0.5 \%$ Triton $\mathrm{X}$ 100. The plates were incubated at room temperature until staining was visible (around 2-3 hours). Selected plants were rescued by transferring to fresh MS medium and kept there for three days. Thereafter, they were transferred to a peat-based, fertilized potting mixture and grown in the greenhouse.

\section{Identification of causal mutations}

The selected mutants, in Col-0 background, were crossed with Ler-1. A dominance test was performed by GUS staining of the F1 and F2 seedlings. To select the plant pools for map-basedcloning, around 2,000 F2 seeds of each mutant x Ler-1 cross were sown on agar plates containing half-strength MS medium with $20 \mathrm{mg} / \mathrm{L}$ Hygromycin. After 5 to 7 days, resistant seedlings containing the $p Z I P 4:: G U S$ construct were transferred to medium with or without $\mathrm{Zn}$, depending on their phenotype. After two weeks a small piece of the root of each plant was collected for GUS staining to determine their phenotype. Depending on the mutant, selected plants were not expressing GUS under Zn deficiency (for mutants found not to express GUS under Zn deficiency) or expressing GUS under Zn sufficiency (for mutants expressing GUS under $\mathrm{Zn}$ sufficiency). The selected seedlings were transplanted to a peat-based potting soil and kept in the greenhouse. Plant material (3 to 4 flower heads) from all plants of each pool was collected for DNA extraction. The DNA was extracted using the DNeasy 96 Plant Kit (Qiagen www.qiagen.us/). The mutant sequences were determined by high-coverage whole-genome sequencing following the SHOREmap method as described by Schneeberger et al. (2009).

\section{Confirmation of mutations and T-DNA insertion lines}

To confirm the mutated sites narrowed down by the SHOREmap, DNA was amplified with the Phusion Flash High-Fidelity PCR Master Mix (Thermo Scientific; www.thermofisher.nl/en/home.html) (Supplemental Table S1). PCR products were Sangersequenced (GATC-BIOTECH; www.gatc-biotech.en/index.html). T-DNA insertion lines were genotyped to confirm or select for homozygous insertion plants. Primers were designed using the website of the Salk Institute (www.signal.salk.edu/tdnaprimers.2.html) (Supplemental Table S2).

\section{Quantification of N-terminal protein acetylation}

This assay was performed as detailed in Linster et al. (2015). 


\section{Quantitative real-time PCR}

Total RNA was extracted from leaves using the Direct-zolTM RNA MiniPrep Kit (Zym o Research, www.zymoresearch.) and cDNA was synthetized with the iScript cDNA Synthesis Kit. Gene expression was quantified by qRT-PCR with SYBR ${ }^{\circledR}$ Green mix (Bio-Rad, www.bio-rad.). Primers for qRT-PCR are detailed in (Assunção et al., 2010).

\section{Transcriptome determination}

For transcriptome analysis, full root systems and shoots of two plants were collected per biological replicate after growing plants for 12 days in hydroponic conditions. Three biological replicates were sampled in each treatment. Total RNA was extracted as described before. RNA quality was confirmed by capillary electrophoresis on an Agilent 2,100 bioanalyzer (Agilent; www.agilent.home). A 250 300 bp insert cDNA library was prepared and sequenced in a HiSeq-S50 Illum ina platform $\mathrm{Q} \geq 90 \%$ (Novogen; en.novogene.). Read quality was checked with FastQC , no trimming was applied, the analysis was done with more than 10 million reads per sample. Reads were aligned to the TAIR 10 version of the Arabidopsis whole genome sequence (Swarbreck et al., 2008) (www.arabidopsis.org/) using STAR (Dobin et al., 2013) (www.genome.gov/encode/). After read alignment, only uniquely aligned reads were kept. Gene expression was quantified using HTSeq (Anders et al., 2015) (htseq.readthedocs.io/en/release_0.9.1/index.html) per exon, where all exons of a gene were used to quantify the overall gene expression level. Only genes that were counted more than once in at least six of the 36 samples were included in the analysis. Gene expression was normalized using the voom method (Law et al., 2014). Mean difference plots were made using $\log _{2}$ transformed count per million reads. To test for significant differences between treatments we performed linear models comparing control treatment with Fe or $\mathrm{Zn}$ deficient treatment for both root and shoot samples. For this analysis, we used eBayes lmFit method (Smyth Gordon, 2004). To test for significant differences between genotypes we performed a pairwise comparison for both root and shoot samples We denoted genes to be significant if they had a False Discovery Rate (FDR) adjusted p-value lower than 0.05 and an absolute log fold change of 0.5 . The sets of genes differentially expressed were analysed for overrepresented Gene Ontology (GO) terms. The GO enrichment analyses were performed using the Database for Annotation, Visualization and Integrated Discovery (DAVID) Bioinformatics Resources 6.8 (Huang et al., 2008, 2009). 


\section{Statistical analysis}

One way ANOVA, two way ANOVA and their respective post hoc test were calculated with Genstat 18th edition (VSN International; www.vsni.co.uk/software/genstat/).

\section{Results}

\section{Identification of $\mathrm{Zn}$ homeostasis mutants}

We designed a Zn-deficiency specific marker system to identify mutants disturbed in $\mathrm{Zn}$ homeostasis. The marker is based on the activity of the Arabidopsis ZRT-IRT-LIKE PROTEIN 4 (ZIP4) Zn transporter promoter. ZIP4 expression is very low under $\mathrm{Zn}$ sufficiency and strongly induced upon Zn deficiency, mainly in roots, but also in shoots (van de Mortel et al., 2006; Assunção et al., 2010; Lin et al., 2016). The full-length promoter was fused to the coding sequence of the $B$-GLUCURONIDASE (GUS) gene to create a $p Z I P 4: \because G U S$ construct (Lin et al., 2016). One transgenic line containing the pZIP4::GUS construct at one locus of insertion, but with multiple T-DNA copies, as determined by Southern hybridization (data not shown), was selected based on stable expression of GUS over at least two generations and exclusively under $\mathrm{Zn}$ deficient conditions (Figure 1).

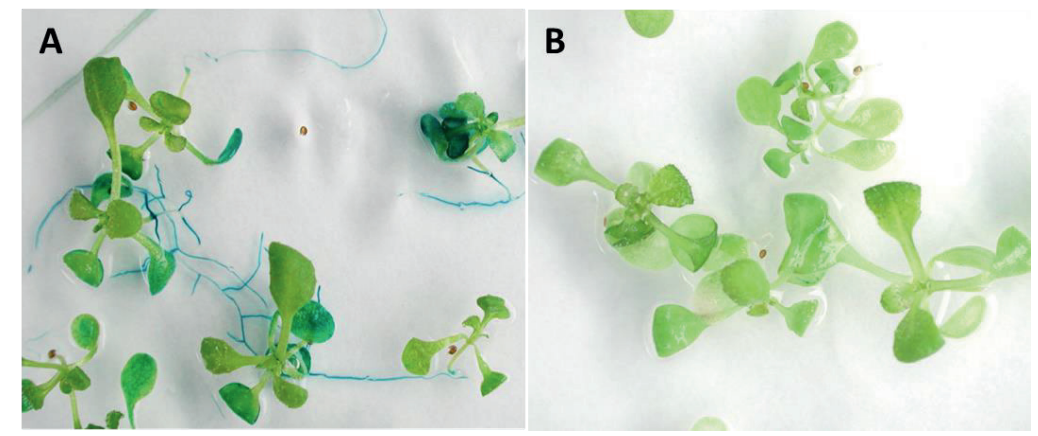

Figure 1. In vivo GUS staining assay of the Arabidopsis Zn deficiency reporter line carrying the pZIP4::GUS reporter construct. Seedlings were grown in MS-agar plates either at (A) Zn deficiency or (B) Zn sufficiency.

The Arabidopsis $p Z I P 4:: G U S$ reporter line was used as the genetic background to generate a $\gamma$-ray-mutagenized M2 population for mutant screens. From $\sim 10000$ irradiated M1 seeds, a large population of $\sim 100$ pools of M2 progeny was obtained. In total, $\sim 35000$ M2 seedlings were screened for aberrant GUS staining, either on Zn sufficiency or Zn deficiency MS agar plates (Supplemental Figure S1). A total of 81 plants were identified to show an aberrant GUS 
staining phenotype. All putative mutant plants were transferred to soil, with 29 M2 plants surviving the transfer and producing M3 progeny by selfing. Since the absence of GUS staining can easily be caused by deletion or mutation of the T-DNA integration, the M3 was tested for hygromycin resistance. Indeed, in 14 out of 29 M3 progenies, the hygromycin resistance was lost. GUS expression in the remaining 15 lines was evaluated on $\mathrm{Zn}$ sufficient and $\mathrm{Zn}$ deficient medium, confirming the M2 mutant phenotype in only four M3 lines (Figure 2), the remaining lines assumed to be false positives. Lines M31 and M68 did not show GUS staining in the Zn deficiency treatment, while lines M22 and M60 expressed GUS in the Zn sufficiency control.

GUS transcript levels were significantly higher under Zn deficiency compared to Zn sufficiency in the pZIP4::GUS background control and in the mutant lines M22 and M60, which was consistent with the GUS staining (Figure 2A and B). The ZIP4 transcript levels were significantly higher in all mutants under $\mathrm{Zn}$ deficiency compared to $\mathrm{Zn}$ sufficiency, with significantly lower transcription in M31, compared to the other lines (Figure 2C). Mutant lines M31 and M68 were expected to have low ZIP4 and GUS transcript levels under Zn deficiency compared to the background control, but this was not the case for the ZIP4 transcripts in M68. Based on the inconsistent expression in M68, we sequenced the construct in this line and discovered a deletion in the construct removing most of the GUS genes, explaining the absence of GUS staining under Zn deficiency. This line was discarded from further analyses. In contrast to expectations, the GUS and ZIP4 transcript levels in M22 and M60 under Zn sufficiency were only marginally, and not significantly, higher than in the $p Z I P 4:: G U S$ background control, even though the GUS staining phenotype was confirmed consistently in at least 60 seedlings per genotype and tested over several generations. 
A

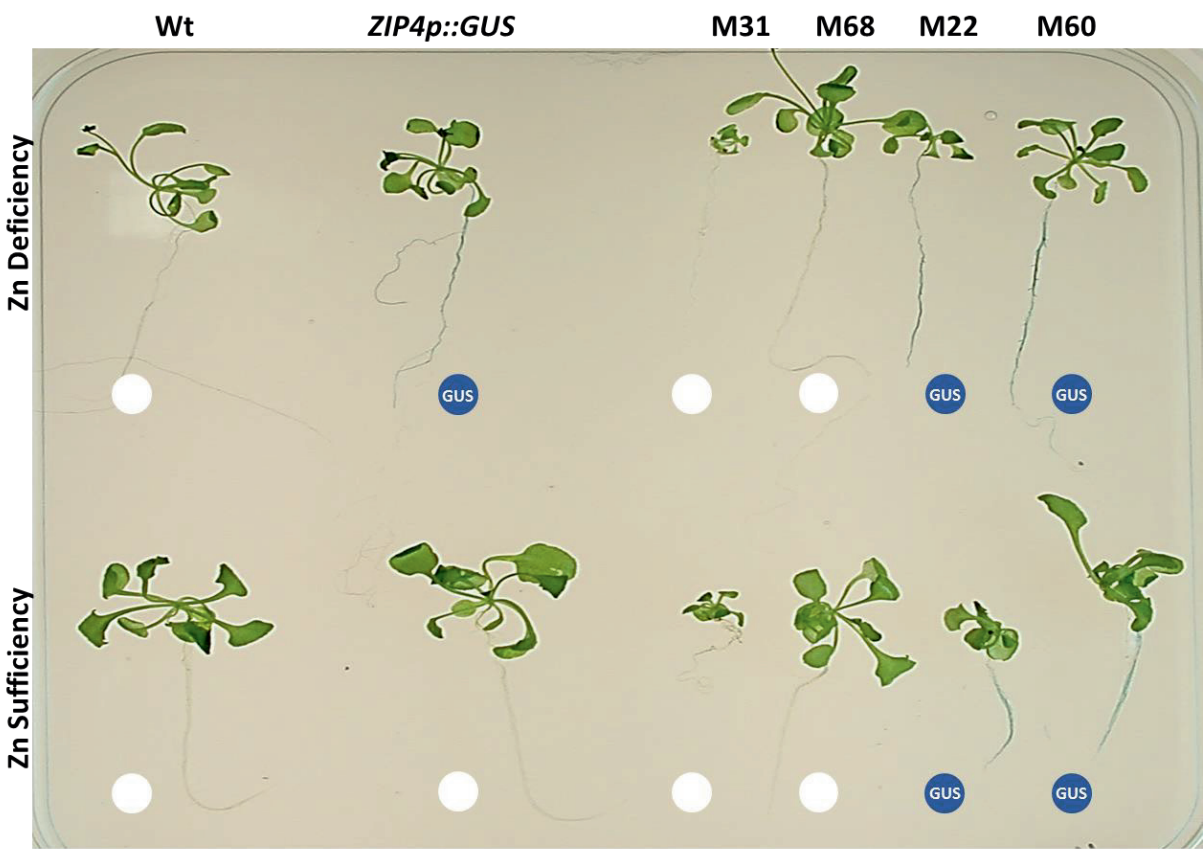

B

\section{C}
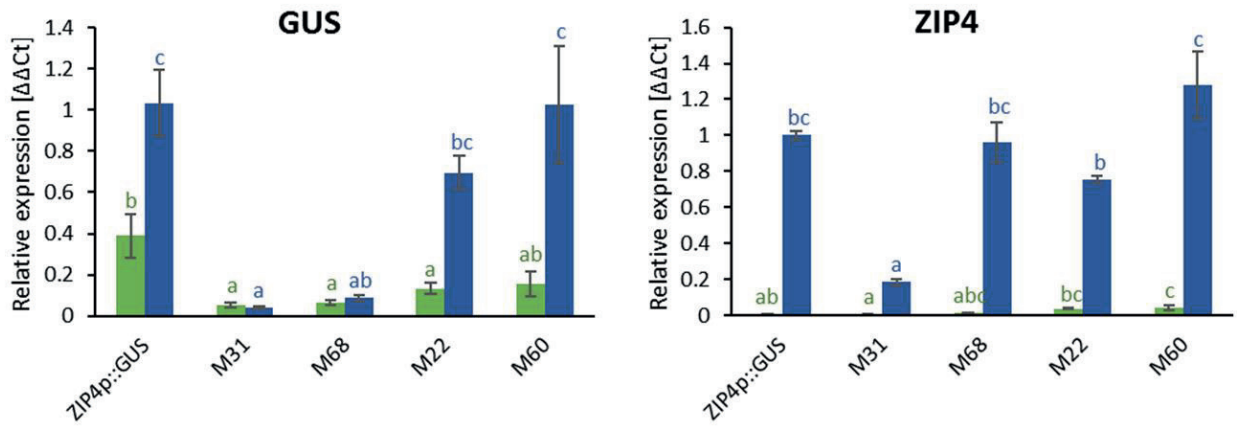

- Suf $\square$ Def

घuf $\square$ Def

Figure 2. Selected mutant lines M31, M68, M22 and M60, displaying an aberrant GUS staining phenotype under either Zn deficiency (M31, M68) or Zn sufficiency (M22, M60). (A) GUS staining phenotypes of wild-type Col-0 (Wt) and the transgenic pZIP4::GUS line used as background for the mutant screen and the four selected mutants grown on vertical MS-agar plates under Zn deficiency (top) and sufficiency (bottom). Expression of (B) GUS and (C) ZIP4 relative to $18 S$ ribosomal RNA in the four mutants and the pZIP4::GUS mutant background, grown under Zn deficiency (Def, blue bars) or Zn sufficiency (Suf, green bars). Means and error bars were calculated from pooled seedlings from three plates. Letters above the bars denote statistically different groups when comparing among genotypes grown under Zn deficiency (blue) or sufficiency (green), obtained with a Tukey post hoc test ( $\alpha=0.05$ ), after a one-way ANOVA (Supplemental Table S3). 

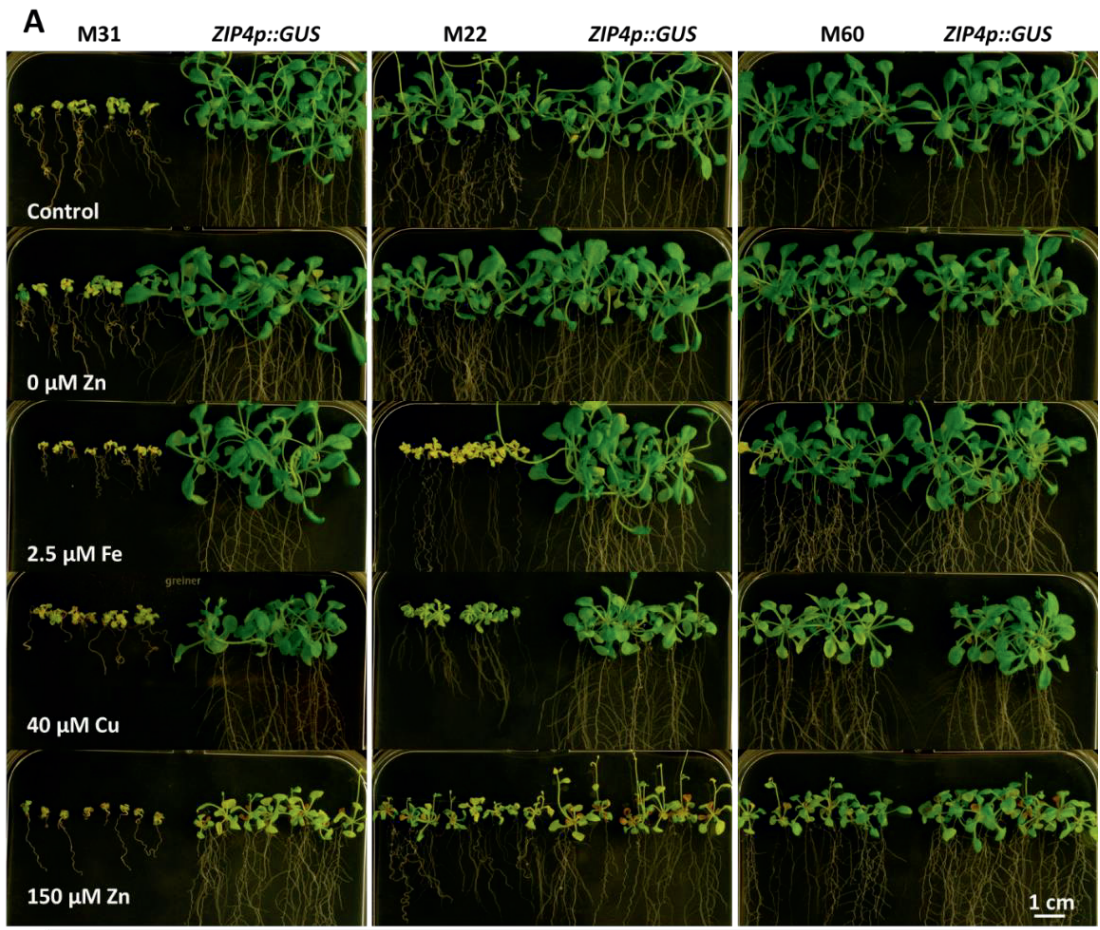

B

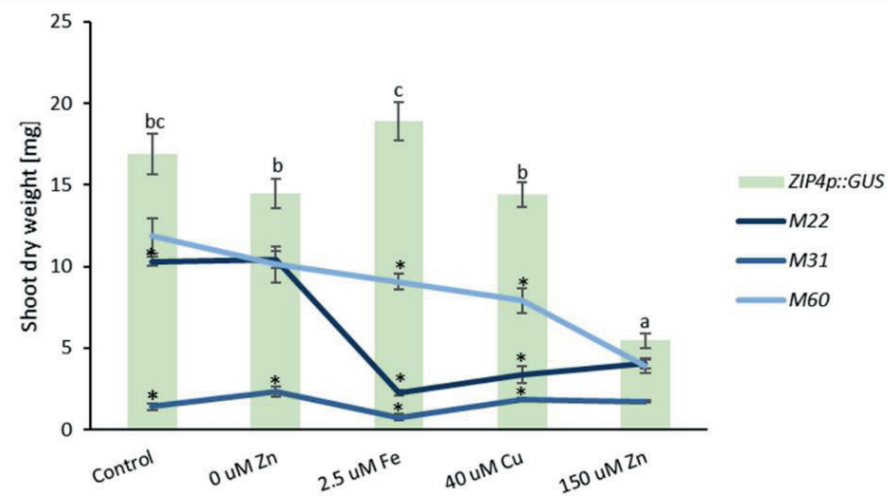

Figure 3. Response of mutant lines M31, M22, and M60 to different mineral stress conditions. (A) Threeweek-old seedlings grown on vertical half MS-agar plates either at Zn or Fe deficiency or at $\mathrm{Cu}$ or Zn excess. (B) Shoot dry weight of the pZIP4::GUS control (green bars), mutant M22 (dark blue line), mutant M31 (medium blue line), and mutant M60 (light blue line). Means and error bars were calculated from pooled seedlings from four plates of each treatment for the mutant lines and from twelve plates of each treatment for pZIP4::GUS control background. Letters above the bars denote statistically different groups of the pZIP4::GUS genotype between treatments, obtained with a Bonferroni post hoc test $(\alpha=0.05)$, after a one-way ANOVA. $\left(^{*}\right)$ above error bars indicates significant differences between the mutant and the pZIP4::GUS control, obtained with a Bonferroni post hoc tests $(\alpha=0.05)$, after a twoway ANOVA (Supplemental Table S4). 


\section{Identification of causal mutations}

To identify the causal mutations of the M22, M31 and M60 mutants, we applied a SHOREmap approach (Schneeberger et al., 2009). First, we crossed the mutants to the Landsberg erecta (Ler) accession and determined that the mutations in all three lines were recessive, based on the absence of the aberrant GUS staining phenotype in F1 seedlings and the recessive segregation ratio of the phenotype in the F2. Subsequently, we selected all hygromycin resistant F2 plants with the corresponding mutant GUS phenotype of each mutant line. Finally, we pooled these selected mutant plants of each F2 progeny for genome sequence analysis, to determine enrichment of genomic sequences corresponding to Col-0 markers, covering the region of the mutation as well as the region of the pZIP4::GUS T-DNA insertion.

The pZIP4::GUS construct insertion was mapped to chromosome 5, between positions 19,236,175 and 19,236,228, disrupting the gene At5g47430, which encodes a CCHC-type zinc finger transcription factor of further unknown function. For the M60 mutant, we did not obtain a clear interval within which the mutation would reside, it looks like some of the pooled plants were collected as false-positive mutants, thus disturbing the analysis. The mutations of M22 and M31 were indicating similar regions on chromosome 5. The interval mapping for M31 highlighted the region between positions $22.5-24.5 \mathrm{Mb}$ as most enriched in Col-0 markers. However, in this interval, there was not a specific region with strong evidence of being associated with the mutant phenotype. Manual examination of the sequence to identify any possible mutation was not successful. The interval mapping for the M22 mutation candidate identified the region between positions 19.5 to $24.5 \mathrm{Mb}$. By comparing the genome sequence with that of Col- 0 and the other mutants, we detected three evident sequence changes. Only one could be confirmed upon resequencing of the specific site. It constitutes an 8-bp deletion in exon 12 of the NAA25 gene (At5g58450) (Supplemental Figure S2). NAA25 encodes the auxiliary subunit of the acetylation complex NatB. The deletion introduces a premature stop codon, roughly in the middle of the NAA25 protein coding sequence. A second mutation was confirmed by sequencing, involving a $G$ to $A$ mutation, within the coding sequence of At5g61940, encoding a ubiquitin carboxyl-terminal hydrolase-related protein, however, this change is outside the mapping interval $(24.8 \mathrm{Mb})$ and thus considered to be less likely to be causal for the mutant phenotype. 
Based on the unambiguous mapping of the M22 mutation, we focused on the two genes in which mutations were found, NAA25 and AT5G61940. Two independent T-DNA insertion lines, for both NAA25 and AT5G61940 (Supplemental Table S2) were verified and grown to establish their phenotypes. Neither of the two T-DNA insertion lines for At5G61940 showed any similarities to the M22 phenotype. This in contrast to the T-DNA insertion lines for NAA25, with insertions in exons 11 (SK24830) and 15 (GK-819A05) of NAA25 respectively. Both showed similar phenotypes as M22 (Figure 4). When compared to wild type, the mutants were shorter; with an increased number of inflorescences originating from the rosette (Figure 4A); chlorotic cauline leaves; wider and curly rosette leaves; chlorotic and aborted siliques (Figure 4B); and malformed flowers with chlorotic sepals (Figure 4C). The two T-DNA insertion lines also showed the same high sensitivity to Fe deficiency as M22 (Figure 4D). To confirm allelism, a cross was made between M22 and SK24830 and between pZIP4::GUS and SK24830. The F1 plants of the first cross all showed the same sensitivity to Fe deficiency as seen in both parents, while the F1 with $p Z I P 4:: G U S$ displayed a normal wild-type phenotype (Supplemental Figure S3). This confirms that the M22 and SK24830 mutants are allelic and their mutant phenotypes are the result of mutations in the same gene, NAA25. In addition to the similar phenotypes, we checked if our three naa25 mutant lines (SK24830, GK-819A05 and M22) were truly affecting the N-terminal acetylation of proteins. We used as a positive control naa20, a mutant for the catalytic subunit of NatB, and compared to the three naa 25 mutant lines (Figure 5). The four mutants behave very similar showing around a 30\% decrease in protein $\mathrm{N}$-terminal acetylation. From this point on we continue only with the SK24830 mutant, which has the disruptive T-DNA insertion in NAA25 very close to the M22 mutation, and is genetically cleaner than M22, as the latter is shown to contain additional mutations and may carry many more, which are not related to the $\mathrm{Zn}$ and Fe homeostasis phenotype, but which may disturb the analysis of the NAA25 gene function. 


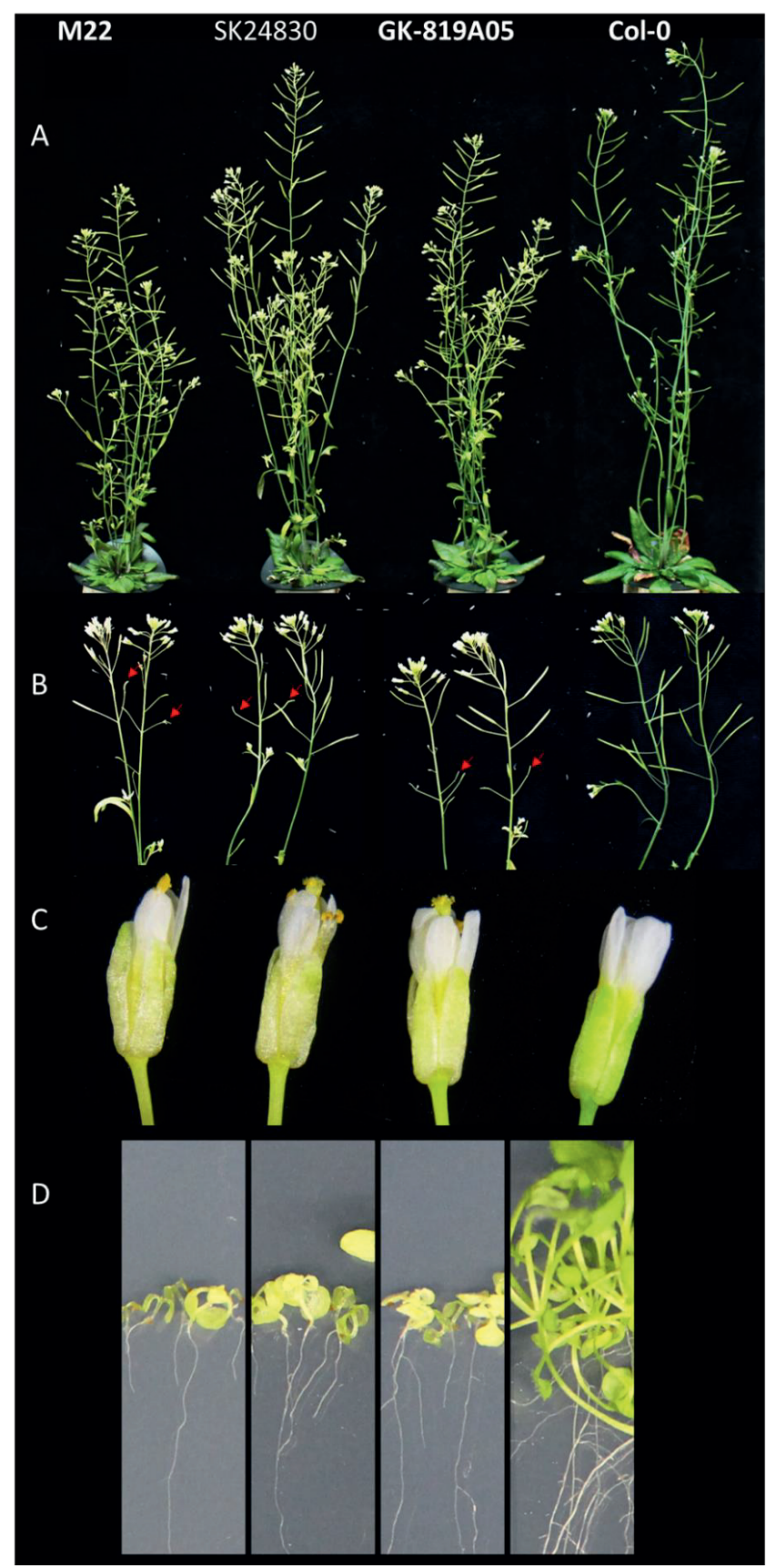

Figure 4. Phenotype of M22, two homozygous NAA25 T-DNA insertion lines carrying the T-DNA in resp. exon 11 and 15 of NAA25 (SK24830 and GK-819A05), and Arabidopsis wild-type plants (Col-0). (A) Fully developed, two-month-old plants, (B) close up of inflorescence, (C) nearly opened flowers, and (D) seedlings grown for three weeks on vertical half MS-agar plates under Fe deficiency. 


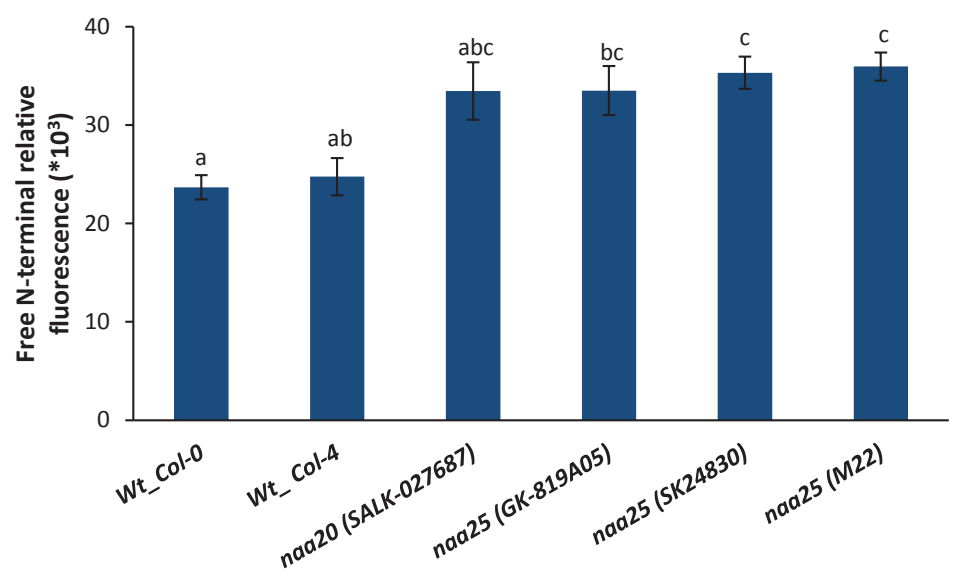

Figure 5. Quantification of free $N$ termini in soluble protein fraction of wild-type, naa20 mutant (Salk027687), a three different naa25 mutant (GK-819A05, SK24830 and m22) plants. Mean $\pm S E, n=4$ replicates. Letters above the bars denote statistically different groups, obtained with a Bonferroni post hoc test $(\alpha=0.05)$, after a one-way ANOVA $(p<0.001)$.

\section{Disruption of $N A A 25$ affects expression of genes in a range of biological processes}

The disruption of NAA25 causes several morphological defects, as well as the disturbance of $\mathrm{Zn}$ and Fe homeostasis. To gain insights into all biological processes that may be disturbed in the naa 25 mutant, we determined the whole transcriptome gene expression profile of naa25 mutant SK24830 and its Col-4 wild-type genetic background by RNA-seq. The naa25 mutant did not show NAA25 transcripts after exon eleven (Supplemental Figure S4). Gene expression was quantified in roots and shoots independently, and denoted to be significantly different between mutant and wild type at a False Rate Discovery (FRD) adjusted p $<0.05$ and an absolute $\log 2$ Fold Change (FC) of 0.5 (which corresponds to a $\mathrm{FC} \geq 1.4$, either up or down). We found 436 genes to be differentially expressed in shoots and 99 differentially expressed genes in roots. Most of the differentially expressed genes were higher expressed in naa25 (Figure 6 and Table 1). From all the genes differentially expressed in naa25, only 14 genes were differentially expressed in both shoots and roots. 


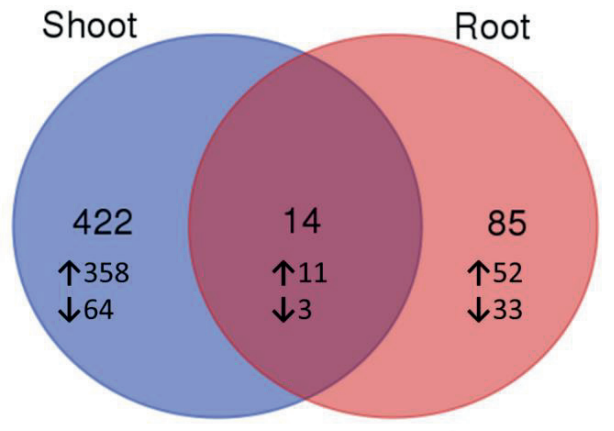

Figure 6. Differential gene expression between the naa25 mutant and the Col-4 wild type (Wt). Venn diagram of the number of differentially expressed genes in roots and shoots between both genotypes ( $\uparrow$ and $\downarrow$ indicating higher and lower expression in naa 25 vs Col-4).

Table 1. Differentially expressed genes in naa25 mutant compared to the Col-4 wild type in both shoots and roots. All differentially expressed genes found in both shoots and roots are shown organized form the ones with higher FC (Fold Change) to the ones with the lower FC.

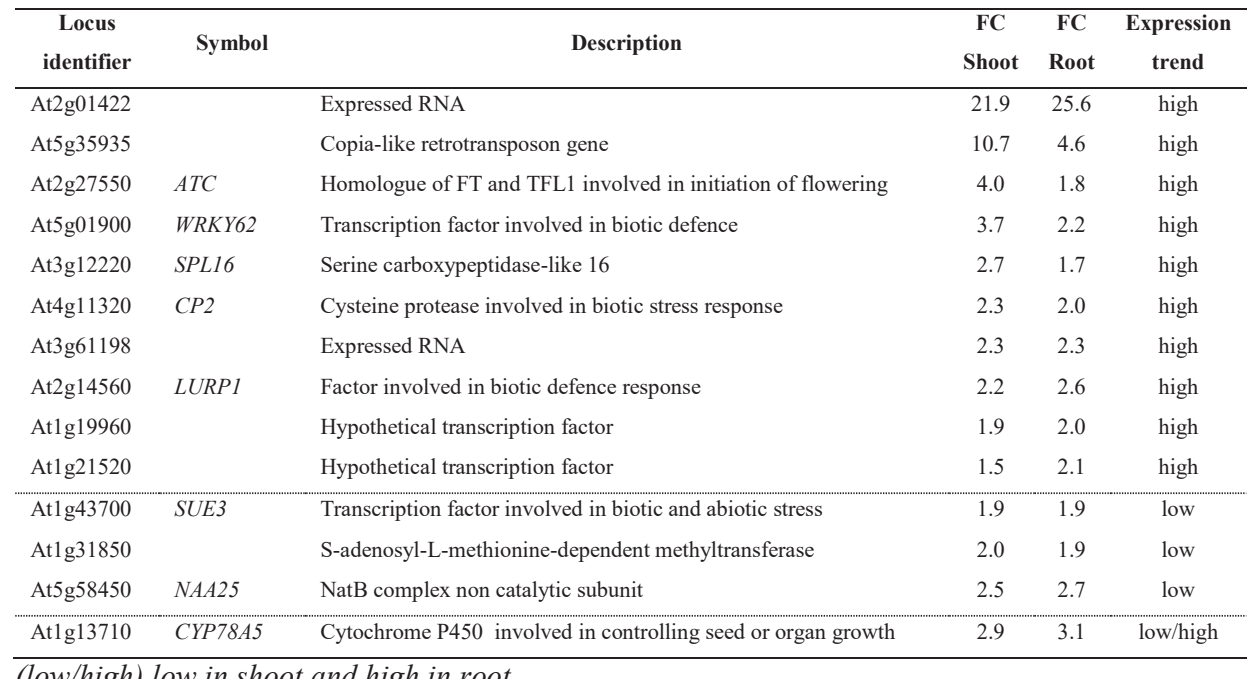

(low/high) low in shoot and high in root

Among the fourteen naa 25 differentially expressed genes found in both roots and shoots (Table 1), several are involved in plants defence. The transcription factor VIRE2-INTERACTING PROTEIN (VIP1) is also known as SULPHATE UTILIZATION EFFICIENCY 3 (SUE3), because sue3 plants with a non-functional allele of this gene are efficient in sulphate uptake (Wu et al., 2010). SUE3 is also a regulator of osmosensory signaling and regulates PATHOGENESIS-RELATED PROTEIN 1 (PRI) expression (Djamei et al., 2007; Tsugama et 
al., 2012). WRKY62 is a negative regulator of plant basal defence, by reducing the expression of PRI (Kim et al., 2008). LATE UPREGULATION IN RESPONSE TO HYALOPERONOSPORA PARASITICA (LURP1) gene shares promoter sequences with the PRI regulon. LURPI is induced by fungal infections and salicylic acid (SA), and it is a negative regulator of cell death during hypersensitive response (HR) (Knoth and Eulgem, 2008; Lee et al., 2017). CP2 is a cysteine protease induced by wounding and involved in plant-mediated RNAi against herbivorous insects (Mao et al., 2013). The transcript of AT1G21520 is induced in response to SA and correlates with genes expressed in plant defence responses and systemic acquired resistance (SAR) (Meier et al., 2008). The transcript of AT3G61198 is down-regulated in light-treated seedlings and shows a negative correlation with transcript levels of SUE3 and a positive correlation with transcript levels of SCN1. The transcript AT2G01422 is upregulated by fungal infections and down-regulated by ABA (Hruz et al., 2008). In total, eight of the fourteen genes differentially expressed in both roots and shoots of naa25 are related with biotic defence responses in plants.

Table 2. Gene Ontology (GO) terms enrichment of differentially expressed genes in the naa25 mutant compared to Col-4 wild-type plants grown in control medium. Top five GO terms enriched within shoots (upper panel) and all GO terms enriched within roots (lower panel) based on higher (high) and lower (low) expressed genes in naa25 compared to wild type. The percentage of query genes over the total number of genes of each biological process is indicated as (\%). Go terms identified using DAVID Bioinformatics Resources 6.8 (david.ncifcrf.gov/).

\begin{tabular}{|c|c|c|c|c|c|}
\hline Biological process & GO code & $\begin{array}{c}\text { Expression } \\
\text { trend }\end{array}$ & p value & $\%$ & $\begin{array}{c}\text { Fold } \\
\text { enrichment }\end{array}$ \\
\hline \multicolumn{6}{|l|}{ Shoot } \\
\hline Anthocyanin-containing compound biosynthetic process & 0009718 & high & $6.5 \mathrm{E}-06$ & 1.7 & 21.4 \\
\hline Oxidation-reduction process & 0055114 & high & $2.9 \mathrm{E}-04$ & 10.2 & 1.9 \\
\hline Response to salicylic acid & 0009751 & high & $4.9 \mathrm{E}-04$ & 2.8 & 4.4 \\
\hline Phloem or xylem histogenesis & 0010087 & high & $7.1 \mathrm{E}-04$ & 1.4 & 12.1 \\
\hline Response to jasmonic acid & 0009753 & high & $2.2 \mathrm{E}-03$ & 2.5 & 3.9 \\
\hline Cytokinin-activated signalling pathway & 0009736 & low & $8.0 \mathrm{E}-04$ & 6.0 & 21.6 \\
\hline Response to chitin & 0010200 & low & $5.9 \mathrm{E}-03$ & 6.0 & 10.7 \\
\hline Cell wall biogenesis & 0042546 & low & $1.2 \mathrm{E}-02$ & 4.5 & 17.8 \\
\hline Cellular zinc ion homeostasis & 0006882 & low & $1.4 \mathrm{E}-02$ & 3.0 & 142.3 \\
\hline Regulation of transcription & 0006355 & low & $2.7 \mathrm{E}-02$ & 17.9 & 2.0 \\
\hline \multicolumn{6}{|l|}{ Root } \\
\hline Salicylic acid mediated signalling pathway & 0009863 & high & $1.2 \mathrm{E}-03$ & 4.9 & 57.7 \\
\hline Metal ion transport & 0030001 & high & $1.9 \mathrm{E}-02$ & 4.9 & 13.9 \\
\hline Oxylipin biosynthetic process & 0031408 & high & $4.6 \mathrm{E}-02$ & 3.3 & 41.7 \\
\hline Aromatic compound biosynthetic process & 0019438 & low & $3.0 \mathrm{E}-04$ & 7.9 & 112.1 \\
\hline Response to osmotic stress & 0006970 & low & $1.9 \mathrm{E}-02$ & 7.9 & 13.8 \\
\hline
\end{tabular}


To obtain a general overview of the biological processes affected in naa25, we performed a GO term enrichment analyses on all differentially expressed genes of plants grown in control medium. Several of the biological processes, for which enrichment was found, are related with biotic defence (Table 2). In addition, genes involved in cellular $\mathrm{Zn}$ homeostasis in shoots and metal ion transport in roots were also differentially expressed in naa25. Enrichment was also found for plant hormone signal transduction (Table 2 and Supplemental Figure S5). Most of the genes enriched in this pathway were lower expressed in naa25.

\section{Mutation of $N A A 25$ alters nutrients homeostasis regulation}

The M22 mutant line was initially selected based on the observed ZIP4 promoter-mediated GUS misexpression under Zn sufficiency, and three independent naa25 mutants were highly sensitive to Fe deficiency. Differentially expressed genes are also enriched for cellular $\mathrm{Zn}$ homeostasis in shoots and metal ion transport in roots, which was why we also examined the whole transcriptome gene expression profile of the naa 25 mutant and its Col-4 genetic wildtype background under $\mathrm{Fe}$ and $\mathrm{Zn}$ deficiency conditions. In naa25 mutant and wild type, both treatments triggered the known deficiency response. The Fe deficiency causes the differential expression of 821 genes in shoots and 512 in roots of both genotypes. This growing conditions upregulated the expression of genes encoding for the transcription factors which regulate the Fe deficiency cascade response. Also, genes encoding for Fe transporters, Fe chelators, and Fe chelate reductases were upregulated in both genotypes in response to $\mathrm{Fe}$ deficiency (Supplemental Table S6). The Zn deficiency causes the differential expression of 54 genes in shoots and 811 in roots in both genotypes. The genes that were strongly induced upon $\mathrm{Zn}$ deficiency encoded for $\mathrm{Zn}$ transporters and $\mathrm{Zn}$ chelators (Supplemental Table S7). This confirmed the effectiveness of the Fe and $\mathrm{Zn}$ deficiency treatments. Large-scale differences between genotypes (naa25 and wild type), treatments (Fe deficiency, Zn deficiency, and control medium), and organs (roots and shoots) were visualized with a multidimensional scaling of all expressed genes. The largest difference was found between root and shoot, then between treatments, and the smallest differences were between genotypes (Supplemental Figure S6). To identify which genes were differentially expressed between the naa25 mutant and the wildtype in each condition tested, we applied a pairwise comparison. As a result, more genes were upregulated in the naa25 mutant compared to the wild type (Table 3). 
Table 3. Number of genes differentially expressed per genotype, treatment, and tissue. Genes differentially expressed in naa25 each grown condition are indicated as the total number, higher (high) or lower (low) expressed genes in naa25 compared to wild type in the column of genotype. Genes differentially expressed in Fe deficiency or Zn deficiency, in both genotypes, compared to control conditions are indicated as the total number, upregulated (up) or downregulated (low) genes in deficiency compared to control medium in the column of treatment. Only the number of significantly differentially expressed genes are shown, based on a False Discovery Rate (FDR) adjusted $p>0.05$ and an absolute $\log _{2}$ fold change of 0.5 .

\begin{tabular}{ccc}
\hline Sample & Genotype (high/low) & Treatment (up/down) \\
\hline Control (shoot) & $436(369 / 67)$ & \\
Control (root) & $99(61 / 38)$ & \\
Fe deficiency (shoot) & $10(4 / 6)$ & $512(598 / 223)$ \\
Fe deficiency (root) & $289(213 / 76)$ & $456(279 / 177)$ \\
Zn deficiency (shoot) & $54(46 / 8)$ & $238(173 / 65)$ \\
Zn deficiency (root) & $811(30 / 510)$ & \\
\hline
\end{tabular}

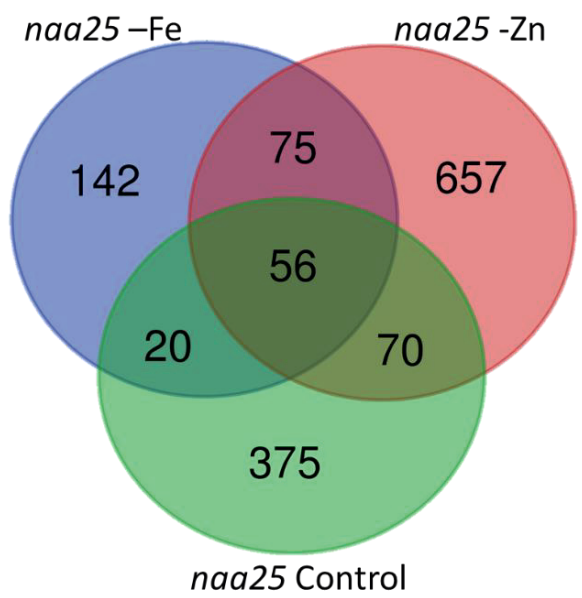

Figure 7. Differentially expressed genes in naa25 mutant upon control, and Zn deficiency treatments. Venn diagram of number differentially expressed genes in naa25 mutant compared to wild type upon control, Zn deficiency (Zn-) or Fe deficiency (Fe-) medium in shoots and roots. Only the number of significantly differentially expressed genes are shown, based on a False Discovery Rate (FDR) adjusted $p>0.05$ and an absolute $\log _{2}$ fold change of 0.5 .

The naa 25 mutant differentially expressed, in at least two of the three conditions tested, several genes involved in nutrients homeostasis as SUE3, SULFATE TRANSPORTER 2;1 (SULTR2;1), RESPONSE TO LOW SULFUR 3 (LSU3), LSU4, SULPHUR DEFICIENCY-INDUCED 1 
(SDI1), BUSH-AND-CHLOROTIC-DWARF 1 (BCD1), HIGH AFFINITY K+ TRANSPORTER 5 (HAK5), CATION EXCHANGER 3 (CAX3), CATION/H+ EXCHANGER 16 (CHX16), MAGNESIUM TRANSPORTER CORA-LIKE, PHOSPHATE 1 (PHO1), NITRATE TRANSPORTER 2.1 (NRT2.1), HEAVY METAL-ASSOCIATED ISOPRENYLATED PLANT PROTEIN 14 (HIPP14), HIPP29, and HEAVY METAL-ASSOCIATED PLANT PROTEIN 10 (HPP10). All of them are higher expressed in naa25 compared to wild type, except for SUE3, PHO1, HAK5, and CAX3.

A total of 293 genes were differentially expressed in naa 25 mutant compared to wild type upon Fe deficiency (Figure 7). From these 293 genes, 215 genes were higher expressed and 78 lower expressed in naa25 mutant compared to wild type upon Fe deficiency. The first GO term enriched by the genes higher expressed was photosynthesis and by the genes lower expressed was cellular response to Fe (Table 4). Among some of the genes involved in transition metal homeostasis differentially expressed in naa25 compared to wild type exclusively due to $\mathrm{Fe}$ deficiency are FE SUPEROXIDE DISMUTASE 1 (FSD1), IRT3, ZIP3, ZIP8, VACUOLAR IRON TRANSPORTER-LIKE 5 (VTL5), FERRIC REDUCTION OXIDASE 1 (FRO1), BGLUCOSIDASE 42 (BGLU42), MYB72, WRKY46, and HIPP2. A total of 858 genes were differentially expressed in naa25 mutant compared to wild type upon Zn deficiency (Figure 7). From these 858 genes, 301 genes were higher expressed and 515 lower expressed in naa25 mutant compared to wild type upon $\mathrm{Zn}$ deficiency. Among some of the genes involved in transition metal homeostasis differentially expressed in naa25 compared to wild type exclusively due to Zn deficiency are HMA2, IRT2, ZIP10, PLANT CADMIUM RESISTANCE 1 (PCR1), FRO4, FRO5, FRO8, HPP11, HPP13, HIPP3, and HIPP13. In general, several GO terms enriched among genes differentially expressed in naa 25 compared to wild type in $\mathrm{Fe}$ or $\mathrm{Zn}$ deficiency consistently include biological processes involved in biotic defence and mineral homeostasis as also observed in control conditions (Table 2).

The M22 mutant was selected in a control medium not in $\mathrm{Zn}$ deficiency. For this reason, we went back to the naa25 in control conditions and looked there for the gene expression patterns of $\mathrm{Zn}$ deficiency responsive genes. In control medium, the $\mathrm{Zn}$ deficiency responsive genes (van de Mortel et al., 2006) ZIP1, ZIP4, ZIP5, IRT3, PCR1, YSL3, NAS1, and NAS4 showed a consistent higher number of transcripts in shoots and roots of naa25 mutant compared to wild type, while ZIP9, ZIP11, FRD3, and Cadmium Sensitive 1 (CAD1) showed only in shoots (Supplemental Figure S7). These genes are 0.7 times higher expressed in naa25 mutant than 
in the wild type on the average and due to the samples variation, they do not appear to be significantly different.

Table 4. Gene Ontology (GO) terms enrichment of differentially expressed genes in the naa25 mutant compared to Col-4 wild-type plants grown under Zn or Fe deficiency. Top five GO terms enriched within Fe deficiency (upper panel) and Zn deficiency (lower panel) based on higher (high) and lower (low) expressed genes in naa25 compared to wild type. The percentage of query genes over the total number of genes of each biological process is indicated as (\%). Go terms identified using DAVID Bioinformatics Resources 6.8 (david.ncifcrf.gov/).

\begin{tabular}{|c|c|c|c|c|c|}
\hline Biological process & GO code & $\begin{array}{l}\text { Expression } \\
\text { difference }\end{array}$ & P value & $\%$ & $\begin{array}{c}\text { Fold } \\
\text { enrichment }\end{array}$ \\
\hline \multicolumn{6}{|l|}{ Fe deficiency } \\
\hline Photosynthesis & 0015979 & high & $1.3 \mathrm{E}-04$ & 3.8 & 7.1 \\
\hline Metal ion transport & 0030001 & high & $2.5 \mathrm{E}-04$ & 3.3 & 7.9 \\
\hline Defense response to bacterium & 0042742 & high & $1.9 \mathrm{E}-03$ & 4.3 & 4.0 \\
\hline Response to chitin & 0010200 & high & 4.6E-03 & 2.9 & 5.5 \\
\hline Regulation of stomatal movement & 0010119 & high & $5.8 \mathrm{E}-03$ & 1.9 & 10.9 \\
\hline Cellular response to iron ion & 0071281 & low & $2.6 \mathrm{E}-09$ & 9.7 & 54.9 \\
\hline Response to hypoxia & 0001666 & low & $8.9 \mathrm{E}-06$ & 6.9 & 37.3 \\
\hline Oxidation-reduction process & 0055114 & low & 7.3E-04 & 18.1 & 3.1 \\
\hline Response to osmotic stress & 0006970 & low & $6.6 \mathrm{E}-03$ & 5.6 & 10.3 \\
\hline Regulation of flower development & 0009909 & low & $2.3 \mathrm{E}-02$ & 4.2 & 12.7 \\
\hline \multicolumn{6}{|l|}{ Zn deficiency } \\
\hline Response to toxic substance & 0009636 & high & $8.5 \mathrm{E}-06$ & 2.4 & 10.8 \\
\hline Cellular transition metal ion homeostasis & 0046916 & high & $2.1 \mathrm{E}-05$ & 2.4 & 9.4 \\
\hline Oxidation-reduction process & 0055114 & high & $6.0 \mathrm{E}-05$ & 10.2 & 2.1 \\
\hline Response to oxidative stress & 0006979 & high & $2.2 \mathrm{E}-04$ & 3.9 & 3.7 \\
\hline Response to wounding & 0009611 & high & $7.1 \mathrm{E}-04$ & 3.0 & 4.1 \\
\hline Plant-type cell wall organization & 0009664 & low & $1.8 \mathrm{E}-13$ & 3.8 & 10.5 \\
\hline Receptor protein tyrosine kinase signalling & 0007169 & low & $3.0 \mathrm{E}-07$ & 3.0 & 5.8 \\
\hline (1->3)-beta-d-glucan biosynthetic process & 0006075 & low & $2.1 \mathrm{E}-06$ & 1.2 & 25.2 \\
\hline Cell wall organization & 0071555 & low & $4.9 \mathrm{E}-06$ & 4.2 & 3.4 \\
\hline Protein phosphorylation & 0006468 & low & $2.5 \mathrm{E}-05$ & 7.4 & 2.1 \\
\hline
\end{tabular}

\section{Zn and Fe deficiency response regulators as possible targets of NatB complex}

The NatB complex is not known to directly regulate the expression of genes. However, it could modify proteins responsible for regulating gene expression. For that reason, we performed a transcription factor prediction based on the genes differentially expressed upon $\mathrm{Fe}$ or $\mathrm{Zn}$ deficiency compared to control conditions. The transcription factors predicted for Fe deficiency were PIF1, PIF4, ELONGATED HYPOCOTYL 5 (HY5), bHLH34, bHLH104, PYE, and WRKY53. For Zn deficiency, PIF4, HY5, and SEPALLATA 3 (SEP3) were the predicted 
transcription factors. For these transcription factors, we analysed their N-t to determine if they could be targets of acetylation by the NatB complex. The transcription factors bHLH104, WRKY53, PIF1, and PIF4 have putative NatB complex N-t targets. Another major known Fe deficiency regulator is FIT which did not appear in our prediction, but it has the sequence to be a target of the NatB complex. In addition, even bZIP23 and bZIP19 did not appear in our prediction either, they are the only known $\mathrm{Zn}$ deficiency regulators. The N-t of bZIP23 and bZIP19 are putative targets for NatB acetylation.

\section{Discussion}

\section{Pleiotropic effects of $N A A 25$ disruption}

In plants and animals, around $80 \%$ of proteins are N-t-acetylated and about $20 \%$ of those acetylations can be carried out by the NatB complex (Linster et al., 2015; Aksnes et al., 2016). The NatB complex is composed of the catalytic subunit NAA20 and the auxiliary subunit NAA25. The non-catalytic subunit is required for protein-protein interactions and proteinribosome interactions (Liszczak et al., 2013). The M22 mutant, and the T-DNA insertion lines in which NAA25 is disrupted showed a range of morphological mutant phenotypes. These effects are expected due to the large number of putative substrates of NatB, which infer major and widespread effects in the cells (Linster et al., 2015; Aksnes et al., 2016).

Most of the biological processes with differentially expressed genes in naa25 mutant were related with stress response, and mainly biotic stress (Table 2). The first biological process upregulated in naa25 mutant was anthocyanins biosynthesis. Anthocyanins are synthesised in response to biotic or abiotic stress conditions and serve as antioxidants that quench reactive oxygen species (ROS). ROS can severely damage cells, but they are also signalling molecules to activate defence response cascades for instance to nutrient deficiency, mechanical disturbance, or pathogen attack (Mittler, 2002; Kovinich et al., 2014). In the naa25 mutant, several biological processes related with plant defence response against wound and pathogen attack have differentially expressed genes. The pathogen resistance regulator SNC1 is the only confirmed substrate of the NatB complex. One of the splice variants of SNC1 is acetylated by NatB, which modifies its stability (Xu et al., 2015). The sncl mutant plant is small with curly leaves and enhanced autoimmune response (Zhang et al., 2003). Common autoimmune phenotypes are dwarfism and elevated salicylic acid levels (van Wersch et al., 2016). Some of the pleiotropic effects observed in naa25 mutants as curly leaves, increased number of basal 
branches, and decrease height of the primary inflorescence, resemble plants with either autoimmune response or pathogen infections (Korves and Bergelson, 2003; Stes et al., 2015). However, naa25 mutant did not show resistance to Hyaloperonospora arabidopsidis colonization compared to wild type (data not shown).

The cytokinin signalling pathway was the main lower expressed biological process in naa25 mutants when compared to wild type. Cytokinins are plant hormones associated with plant immunity, growth, development and Fe homeostasis (Choi et al.; Mathilde et al., 2008). In addition, to the cytokinin signalling pathway to the naa25 mutant shows to be affected at several levels of the general plant hormone signal transduction (Supplemental Figure S7). The misregulation of genes in these pathways can affect processes related with cell division and cell enlargement, therefore shoot initiation and plant growth. This would be in line with some of the pleiotropic phenotypes of the naa25 mutants, such as branching, and dwarfism. Genes involved in transport tissue histogenesis were higher expressed in naa25 mutant compared to wild type. Members of this biological process are DOT1 and DOT3, mutations on them cause aberrant venation patterns in leaves. The dot 3 plants are small with problems with fertility (Petricka et al., 2008) and their leaf phenotype resembles the leaf phenotype seen in the naa25 mutant (Ferrández-Ayela et al., 2013). Therefore, some of the morphologic defects observed in naa25 mutants could be a consequence of the interferences with the plant hormone signalling pathways.

\section{The disruption of $N A A 25$ affects the regulation of nutrients homeostasis}

Several genes in charge of nutrient homeostasis are misregulated in naa 25 mutant, in both control and $\mathrm{Fe} / \mathrm{Zn}$ deficiency conditions. These genes have functions related to both macro (S, $\mathrm{P}, \mathrm{Mg}, \mathrm{K}$ and $\mathrm{Ca}$ ) and micronutrient transport ( $\mathrm{Zn}, \mathrm{Cu}, \mathrm{B}$, and $\mathrm{Fe}$ ). Particularly, several genes involved S homeostasis are differentially expressed across all the conditions tested. The downregulation of genes involved in $\mathrm{S}$ assimilation was also reported for NatA impaired mutants (Linster et al., 2015). This could be due to the differential expression of SUE in naa 25 compared to wild type, which is likely to be involved in low S sensing (Wu et al., 2010). Furthermore, SUE3 is a putative target of NatB.

In the case of $\mathrm{Zn}$ homeostasis, the increased expression of ZIP4 in control conditions was a common pattern across several $\mathrm{Zn}$ deficiency transporter genes in naa25 mutant. Within this genes, we had several members of the ZIP family and genes from other families which are 
mainly expressed under Zn deficiency conditions (van de Mortel et al., 2006). This supports the initial notion on which the M22 mutant was selected, that the naa25 mutants display a weak $\mathrm{Zn}$ deficiency response under sufficient $\mathrm{Zn}$ supply. The $\mathrm{Zn}$ deficiency response in Arabidopsis is regulated by two transcription factors, bZIP19 and bZIP23 (Assunção et al., 2010). The Nterminus of both bZIP19 and bZIP23 contain sequences that are putative targets of NatB. If bZIP19 and 23 are indeed acetylated by NatB, their regulatory function could be affected by the lack of acetylation in the naa25 mutant. The role of bZIP19 and 23 does not depend on their transcription levels (Assunção et al., 2010). However, their stability, activity, function and/or localization may depend on post-transcriptional modification such as N-t-acetylation, possibly even affected by the $\mathrm{Zn}$ status of the plant. In the case of Fe, the Fe deficiency response regulators bHLH104 and FIT (Yuan et al., 2008; Zhang et al., 2015; Li et al., 2016) are also putative targets of NatB.

\section{Fe deficiency and biotic defence response signalling}

The pathogen defence response and the Fe deficiency response share common processes in plants, such as hormone signalling and the secretion of phenolic compounds. Ethylene, nitric oxide, jasmonic acid and salicylic acid regulate the plant immune response and are also important factors in Fe homeostasis. Several phenolic compounds, production of which is induced upon Fe starvation, have strong antimicrobial properties, next to Fe solubilizing and binding properties. Fe deficiency and biotic defence responses are also evolutionarily linked as soil microorganisms release siderophores (high-affinity iron-chelating compounds) for $\mathrm{Fe}$ scavenging, and their perception by the plant triggers both the Fe deficiency and immune responses (Aznar et al., 2014).

Several genes implicated in this link between Fe homeostasis and immunity, are differentially expressed in the naa 25 mutant when compared to wild type. For instance, the MYB72 gene was lower expressed in the naa25 mutant upon Fe deficiency compared to wild type. It plays a dual role, by regulating some of the early steps in the Fe deficiency response (Palmer et al., 2013) and its involvement in Induced Systemic Resistance (ISR) (Zamioudis et al., 2015) (MartínezMedina et al., 2017). The MYB72 transcription factor induces the expression of BGLU42 which encodes a component of the ISR signalling pathway that is required for the secretion of ironmobilizing phenolic compounds into the rhizosphere under Fe deficiency (Zamioudis et al., 2014). Like $M Y B 72$, also the expression of $B G L U 42$ was lower in the naa 25 mutant upon $\mathrm{Fe}$ deficiency compared to wild type. The transcription WRKY53, which was predicted to be one 
of the regulators the differential gene expression in Fe deficiency for both naa 25 and wild type, is also a putative target of NatB. This transcription factor is involved in pathogen-associated molecular pattern-triggered immunity (Nie et al., 2017). WRKY53 is involved in the salicylic signalling pathway, in which it cross-regulate with WRKY46. The expression of WRKY46 was higher in naa 25 compared to wild type. Its expression significantly affects the root-to-shoot $\mathrm{Fe}$ translocation, (Yan et al., 2016). The Fe deficiency response and the biotic defence response are linked through regulators, interestingly the genes making this link are particularly differentially expressed in naa25 mutant compared to wild type.

\section{Photosynthesis and naa25 mutant sensitivity to Fe deficiency}

One of the most Fe demanding plant processes is photosynthesis, which is why chloroplasts contain around $80 \%$ of the $\mathrm{Fe}$ in leaves. Iron is a crucial element in the electron-transport chains of photosynthesis and respiration, due to its ability to readily accept and donate electrons (Varotto et al., 2002) (Connolly and Guerinot, 2002; Kessler and Papenbrock, 2005; Yruela, 2013). Several genes encoding proteins of the Photosystem II (PSII) complex were differentially expressed upon Fe deficiency. Even more, genes encoding components of PSI and PSII were differentially expressed in the naa 25 mutant under Fe deficiency (Supplemental Table S8).

The photosynthetic activity of a plant drastically decreases under Fe deficiency due to severely photodamaged PSII (Araki and Shikanai, 2014). The PIF1 and PIF4 were predicted as some of the regulators of genes differentially expressed due to Fe deficiency in our study. In addition, the sequences of both proteins are putative targets of acetylation by NatB. In general, PIFs link light signals to the pathway of the phytohormones ethylene, gibberellin, and cytokinin signaling pathways (Zhang et al., 2017) (Liu et al., 2017). Therefore, the lack of acetylation of any of PIF1 or PIF4 could further compromise photosynthesis in the naa25 mutant, especially under Fe deficiency. 


\section{Conclusions}

Although not all targets of N-t-acetylation by the NatB complex are determined as yet, it potentially modifies many proteins involved in many biological processes. Transcript profiling allowed us to get a glimpse of the biological processes which are altered in the naa25 mutant. The major processes that are affected in the naa 25 mutant relate to plant defence response, which is closely linked with Fe deficiency response. This could be the reason of the high sensitivity to Fe deficiency. However, Fe homeostasis does not seem to be the only mineral homeostasis affected. The $\mathrm{Zn}$ deficiency response is slightly induced under $\mathrm{Zn}$ sufficiency conditions, which could be either a pleiotropic effect of the reduced acetylation in the naa25 mutant or the lack of acetylation in a $\mathrm{Zn}$ homeostasis regulator. Regulators controlling the homeostasis of $\mathrm{Fe}$ and $\mathrm{Zn}$ are putative substrates of NatB N-t-acetylation.

\section{Acknowledgements}

We thank Dr. Korbinian Schneeberger (Max Planck Institute) for performing the SHOREmap analysis. We thank Prof. Guido van den Ackerveken and Annemiek Andel (Utrecht University) for performing the disease assay with Hyaloperonospora. This work was supported by the Ecuadorian government through SENESCYT (Secretaría de Educación Superior, Ciencia, Tecnología e Innovación) and Universidad de las Fuerzas Armadas-ESPE. 


\section{Supplemental information}

Table S1. Primers for verification of mutations in AT5G58450 and AT5G61940.

\begin{tabular}{cccc}
\hline Symbol & Locus identifier & Primer & Sequence (5' - 3') \\
\hline \multirow{2}{*}{$N A A 25$} & At5g58450 & Forward & GGCAGAACTGGAAATTGAGA \\
& Reverse & GCTTCAGCAAGATAGCCAAA \\
& \multirow{2}{*}{ At5g61940 } & Forward & AAGTGTTGCAGGCCATAGAC \\
& Reverse & GAAACTGGCCATTGAGGTACT
\end{tabular}

Table S2. T-DNA insertion lines for AT5G58450 and AT5G61940 and primers flanking the left border $(L B)$ and right border (BP) of the insertion, used for their genotyping.

\begin{tabular}{|c|c|c|c|c|}
\hline Symbol & Locus identifier & T-DNA line & Primer & Sequence (5'- 3') \\
\hline \multirow{4}{*}{ NAA25 } & \multirow{4}{*}{ At5g58450 } & \multirow{2}{*}{ SK24830 } & LP & AGCCAAAATCTCGAGTTCTCC \\
\hline & & & $\mathrm{RB}$ & GGCTGTCTGCTTGAAGATGAC \\
\hline & & \multirow{2}{*}{ GK-819A05 } & LP & TGTATTAGGTTGCATCCGTCC \\
\hline & & & $\mathrm{RB}$ & TTTTGGCTATCTTGCTGAAGC \\
\hline & \multirow{4}{*}{ At5g61940 } & \multirow{2}{*}{ GK_348D02 } & LP & AAGGGAGAGCTTGGTAACTCG \\
\hline & & & $\mathrm{RB}$ & GACGTTTAGCTCAAGCTCTGC \\
\hline & & \multirow{2}{*}{ SALK_053216C } & LP & TCTTGCTGGTTGGTTTGAAAC \\
\hline & & & RB & TGGTTGGTTCAGCAAATCTTC \\
\hline
\end{tabular}

Table S3. Results of one-way ANOVA performed for relative gene expression of ZIP4 and GUS transcripts for mutant lines M31, M22, and M60, and their pZIP4::GUS control background grown at Zn sufficiency (Suf) or Zn deficiency (Def).

\begin{tabular}{ccc}
\hline Variable & Variance ratio & F probability \\
\hline ZIP4 - Suf & 5.62 & 0.006 \\
GUS - Suf & 5.86 & 0.005 \\
ZIP4 - Def & 17.27 & $<.001$ \\
GUS - Def & 10.57 & $<.001$ \\
\hline
\end{tabular}


Table S4. Results of two-way ANOVA performed for dry shoot weight, for each mutant line (M31, M22, and M60) and it respectively pZIP4::GUS control background grown at control medium, Zn or Fe deficient or at $\mathrm{Cu}$ or $\mathrm{Zn}$ excess. Letters between brackets denote statistically different groups, when comparing grown medium and genotypes, obtained with a Bonferroni post hoc test $(\alpha=0.05)$, after a two-way ANOVA.

\begin{tabular}{|c|c|c|c|c|}
\hline $\begin{array}{l}\text { Mutant } \\
\text { line }\end{array}$ & Factor & $\begin{array}{l}\text { Variance } \\
\text { ratio }\end{array}$ & F probability & Groups \\
\hline \multirow{3}{*}{ M22 } & Genotype & 496.5 & $<.001$ & M22 2.5 uM Fe (a), M22 40 uM Cu (ab), M22 120 uM Zn (ab), \\
\hline & Treatment & 75.56 & $<.001$ & $\begin{array}{l}\text { ZIP4p::GUS } 120 \text { uM Zn (b), M22 Control (c), M22 } 0 \text { uM Zn (c), } \\
\text { ZIP4p::GUS } 0 \text { uM Zn (cd), ZIP4p::GUS } 40 \text { uM Cu (d), ZIP4p::GUS }\end{array}$ \\
\hline & Interaction & 59.26 & $<.001$ & Control (e), ZIP4p::GUS 2.5 uM Fe (e) \\
\hline \multirow{3}{*}{ M31 } & Genotype & 497.6 & $<.001$ & M31 2.5 uM Fe (a), M31 2.5 uM Fe (a), M31 Control (a), M31 120 \\
\hline & Treatment & 20.43 & $<.001$ & $\begin{array}{l}\text { uM Zn (a), M31 } 40 \text { uM Cu (a), M31 } 0 \text { uM Zn (a), ZIP4p::GUS } 120 \\
\text { uM Zn (a), ZIP4p::GUS } 40 \text { uM Cu (b), ZIP4p::GUS Control (bc), }\end{array}$ \\
\hline & Interaction & 23.55 & $<.001$ & ZIP4p::GUS 0 uM Zn (bc), ZIP4p::GUS 2.5 uM Fe (c) \\
\hline \multirow{3}{*}{ M60 } & Genotype & 60.45 & $<.001$ & M60 120 uM Zn (a), ZIP4p::GUS 120 uM Zn (ab), M60 40 uM Cu \\
\hline & Treatment & 23.47 & $<.001$ & $\begin{array}{l}\text { (abc), M60 } 2.5 \text { uM Fe (bcd), M60 } 0 \text { uM Zn (bcde), M60 Control } \\
\text { (cdef), ZIP4p::GUS } 0 \text { uM Zn (def), ZIP4p::GUS } 40 \text { uM Cu (ef), }\end{array}$ \\
\hline & Interaction & 2.4 & 0.079 & ZIP4p::GUS Control (f), ZIP4p::GUS 2.5 uM Fe (f) \\
\hline
\end{tabular}


Table S5. Most differentially expressed genes between the naa25 mutant and the Col-4 wild type under control growing conditions. Top ten genes with the highest FC Fold Change) either in shoots (top) or in roots (bottom)

\begin{tabular}{|c|c|c|c|c|}
\hline $\begin{array}{c}\text { Locus } \\
\text { identifier }\end{array}$ & Symbol & Description & FC & $\begin{array}{c}\text { Expression } \\
\text { trend }\end{array}$ \\
\hline \multicolumn{5}{|l|}{ Shoots } \\
\hline At3g47050 & & Glycosyl hydrolase protein & 7.2 & high \\
\hline At4g22517 & & Protease inhibitor/seed storage & 5.0 & high \\
\hline At2g39030 & NATAl & $\mathrm{N}-\Delta$-acetyltransferase, induced in response to MeJA and ABA & 3.7 & high \\
\hline At5g64810 & WRKY51 & Transcription factor involved in JA mediated responses & 3.6 & high \\
\hline At4g22513 & & Protease inhibitor/seed storage & 3.6 & high \\
\hline At4g22475 & & Transmembrane protein & 3.3 & high \\
\hline Atlg04660 & & Glycine-rich protein & 4.0 & low \\
\hline \multicolumn{5}{|l|}{ Roots } \\
\hline At2g43920 & HOL 2 & Methyltransferase involved in biotic defence & 4.3 & high \\
\hline At3g12230 & SPL14 & Serine carboxypeptidase-like 14 & 3.4 & high \\
\hline At5g22570 & $W R K Y 38$ & Transcription factor involved in biotic defence response & 3.3 & high \\
\hline At $\lg 15610$ & & Transmembrane protein & 3.2 & high \\
\hline Atlg15620 & & Transmembrane protein & 3.1 & high \\
\hline At3g30260 & $A G L 79$ & AGAMOUS-like 79 & 2.8 & low \\
\hline
\end{tabular}


Table S6. Differentially expressed genes of both shoot and roots when comparing control conditions to Fe deficiency treatment in naa25 and Col-4. Only genes upregulated (up) or downregulated (down) in Fe deficiency with at least a Fold Change (FC) $>3$ in one organ (shoots or roots) and at least a FC $>1.5$ in the second organ with a significance level of $p<0.001$ are listed.

\begin{tabular}{|c|c|c|c|c|c|}
\hline $\begin{array}{c}\text { Locus } \\
\text { identifier }\end{array}$ & Symbol & Description of encoded proteins & $\begin{array}{c}\text { FC } \\
\text { shoot }\end{array}$ & $\begin{array}{c}\text { FC } \\
\text { root }\end{array}$ & $\begin{array}{c}\text { Expression } \\
\text { trend }\end{array}$ \\
\hline At3g56970 & bHLH38 & Transcription factor involved in Fe homeostasis & 23.5 & 6.2 & up \\
\hline At3g56980 & bHLH39 & Transcription factor involved in Fe homeostasis & 21.7 & 4.7 & up \\
\hline At $2 \mathrm{~g} 41240$ & BHLH100 & Transcription factor involved in Fe-deficiency responses & 20.4 & 10.6 & up \\
\hline At1g13609 & & Defensin-like (DEFL) family protein & 14.0 & 22.9 & up \\
\hline At5g04150 & BHLH101 & Transcription factor involved in Fe-deficiency responses & 10.8 & 8.4 & up \\
\hline At2g14247 & & Expressed protein & 10.1 & 3.4 & up \\
\hline At1g13608 & & Defensin-like & 9.6 & 2.8 & up \\
\hline At1g12030 & & Phosphoenolpyruvate carboxylase & 7.7 & 6.1 & up \\
\hline Atlg74770 & BTSL1 & Zinc ion binding & 5.5 & 2.2 & up \\
\hline At4g19690 & IRTI & Iron transporter & 5.0 & 7.1 & up \\
\hline At 1 g24580 & & RING/U-box superfamily & 4.8 & 2.3 & up \\
\hline Atlg23020 & FRO3 & Ferric chelate reductase & 3.8 & 2.3 & up \\
\hline At5g53450 & ORGI & OBP3-responsive protein 1 & 3.4 & 2.5 & up \\
\hline At3g02480 & $A B R$ & Late embryogenesis abundant & 3.0 & 2.6 & up \\
\hline At1g56430 & NAS4 & Nicotianamine synthase & 3.0 & 2.9 & up \\
\hline At5g67370 & $C G L D 27$ & DUF1230 family protein & 2.8 & 5.3 & up \\
\hline At4g16370 & OPT3 & Iron transporter that loads iron into the phloem & 2.3 & 4.1 & up \\
\hline Atlg01580 & FRO2 & Ferric chelate reductase involved in Fe reduction at the root surface & 2.2 & 6.9 & up \\
\hline At3g12820 & $M Y B 10$ & R2R3 factor & 1.6 & 3.1 & up \\
\hline At5g59520 & $Z I P 2$ & Metal ion transporter regulated by copper & 2.4 & 11.2 & down \\
\hline At1g68650 & & Uncharacterized protein family (UPF0016) & 3.2 & 1.8 & down \\
\hline At $2 g 21650$ & $M E E 3$ & Member of MYB transcription factors & 3.4 & 2.3 & down \\
\hline At $2 \mathrm{~g} 40300$ & FER4 & Ferritins protect against oxidative damage & 3.6 & 2.6 & down \\
\hline At1g21140 & $V T L 1$ & Nodulin-like1 & 4.7 & 3.7 & down \\
\hline At4g25100 & FSDI & Fe-superoxide dismutase & 17.8 & 16.3 & down \\
\hline
\end{tabular}


Table S7. Differentially expressed genes of both shoot and roots when comparing control conditions with a Zn deficiency treatment in naa25 and Col-4. Only genes upregulated (up) or downregulated (down) in Zn deficiency with at least a Fold Change (FC) $>3$ in one organ (shoots or roots) and at least a FC $>1.5$ in the second organ with a significance level of $p<0.05$ are listed.

\begin{tabular}{|c|c|c|c|c|c|}
\hline $\begin{array}{c}\text { Locus } \\
\text { identifier }\end{array}$ & Symbol & Description of encoded proteins & $\begin{array}{c}\text { FC } \\
\text { shoot }\end{array}$ & $\begin{array}{l}\mathrm{FC} \\
\text { root }\end{array}$ & $\begin{array}{c}\text { Expression } \\
\text { Trend }\end{array}$ \\
\hline At4g33020 & $Z I P 9$ & ZIP family protein induced in response to $\mathrm{Zn}$ deficiency & 43.8 & 6.0 & up \\
\hline At2g03445 & MIR398A & MicroRNA that targets both CSD and CytC & 23.7 & 23.0 & up \\
\hline Atlg09155 & $P P 2-B 15$ & Phloem protein 2-B15 & 13.9 & 3.5 & up \\
\hline At5g61320 & CYP89A3 & Member of CYP89A & 10.4 & 9.0 & up \\
\hline Atlg20380 & & Prolyl oligopeptidase & 9.3 & 6.9 & up \\
\hline At5g60060 & & F-box SKIP23-like & 8.6 & 3.5 & up \\
\hline Atlg10970 & $Z I P 4$ & ZIP family protein induced in response to $\mathrm{Zn}$ deficiency & 8.0 & 4.7 & up \\
\hline At3g08040 & FRD3 & Transporter involved in root xylem loading of $\mathrm{Fe}$ chelator & 7.3 & 2.5 & up \\
\hline Atlg56242 & & Potential natural antisense gene & 7.1 & 15.5 & up \\
\hline Atlg56240 & $P P 2-B 13$ & Phloem protein 2-B13 & 7.1 & 4.8 & up \\
\hline Atlg60960 & $I R T 3$ & Plasma membrane zinc/iron transporter & 7.0 & 3.1 & up \\
\hline At $2 \mathrm{~g} 37810$ & & Cysteine/Histidine-rich $\mathrm{C} 1$ domain family protein & 6.4 & 2.7 & up \\
\hline Atlg05300 & ZIP5 & ZIP family protein induced in response to $\mathrm{Zn}$ deficiency & 6.0 & 3.3 & up \\
\hline At5g50400 & PAP27 & Purple acid phosphatase 27 & 4.5 & 3.0 & up \\
\hline Atlg09240 & $N A S 3$ & Nicotianamine synthase & 3.4 & 19.2 & up \\
\hline At $3 g 12750$ & $Z I P 1$ & ZIP family protein induced in response to $\mathrm{Zn}$ deficiency & 2.6 & 3.4 & up \\
\hline Atlg56430 & NAS4 & Nicotianamine synthase & 2.2 & 6.4 & up \\
\hline At3g05790 & LON4 & Lon protease-like & 2.1 & 5.3 & up \\
\hline At4g30110 & $H M A 2$ & P1B-type ATPases transport zinc & 1.6 & 3.0 & up \\
\hline At3g04450 & & Homeodomain-like superfamily protein & 1.6 & 5.1 & up \\
\hline At $2 \mathrm{~g} 44290$ & & Lipid-transfer protein/seed storage $2 \mathrm{~S}$ albumin & 1.5 & 3.5 & up \\
\hline At3g01500 & CA1 & Carbonic anhydrase & 2.3 & 6.8 & up/down \\
\hline
\end{tabular}

(up/down) upregulated in shoots and downregulated in roots 
Table S8. Genes involved in photosynthesis that are differentially expressed due to Fe deficiency. Genes differentially expressed upon Fe deficiency in wild type and naa25 (Wt, naa25) compared to control medium, upon Fe deficiency in naa25 (naa25) but not in wild type compared to control medium, upon Fe deficiency in wild type and in naa25 and even more in naa25 (Wt, naa25).

\begin{tabular}{|c|c|c|}
\hline AGI & Gene Name & Genotype \\
\hline Atlg76570 & Chlorophyll A-B binding family protein(AT1G76570) & $\mathrm{Wt}$, naa25 \\
\hline Atlg29920 & Chlorophyll A/B-binding protein 2(CAB2) & $\mathrm{Wt}, \underline{\text { naa } 25}$ \\
\hline At5g08410 & Ferredoxin/thioredoxin reductase subunit A (variable subunit) 2(FTRA2) & $\mathrm{Wt}$, naa 25 \\
\hline At5g01600 & Ferritin 1(FER1) & $\mathrm{Wt}$, naa 25 \\
\hline At3g56090 & Ferritin 3(FER3) & $\mathrm{Wt}$, naa 25 \\
\hline At2g40300 & Ferritin 4(FER4) & $\mathrm{Wt}$, naa 25 \\
\hline At $4 \mathrm{~g} 10340$ & Light harvesting complex of photosystem II 5(LHCB5) & $\mathrm{Wt}$, naa 25 \\
\hline At $\lg 15820$ & Light harvesting complex photosystem II subunit 6(LHCB6) & $\mathrm{Wt}$, naa 25 \\
\hline At2g34430 & Light-harvesting chlorophyll-protein complex II subunit B1(LHB1B1) & $\mathrm{Wt}$, naa 25 \\
\hline At5g13630 & Magnesium-chelatase subunit chlH, chloroplast, putative (CHLH)(GUN5) & $\mathrm{Wt}$, naa 25 \\
\hline At3g14940 & Phosphoenolpyruvate carboxylase 3(PPC3) & $\mathrm{Wt}$, naa 25 \\
\hline At5g64040 & Photosystem I reaction center subunit PSI-N, chloroplast, putative (PSAN)(PSAN) & $\mathrm{Wt}$, naa 25 \\
\hline Atlg03130 & Photosystem I subunit D-2(PSAD-2) & $\mathrm{Wt}$, naa 25 \\
\hline Atlg31330 & Photosystem I subunit F(PSAF) & $\mathrm{Wt}$, naa 25 \\
\hline At2g05100 & Photosystem II light harvesting complex protein 2.1(LHCB2.1) & $\mathrm{Wt}$, naa 25 \\
\hline At $2 \mathrm{~g} 05070$ & Photosystem II light harvesting complex protein 2.2(LHCB2.2) & $\mathrm{Wt}$, naa 25 \\
\hline At3g27690 & Photosystem II light harvesting complex protein 2.3(LHCB2.3) & $\mathrm{Wt}, \underline{\text { naa } 25}$ \\
\hline At4g05180 & Photosystem II subunit Q-2(PSBQ-2) & $\mathrm{Wt}$, naa 25 \\
\hline Atlg79040 & Photosystem II subunit R(PSBR) & $\mathrm{Wt}, \underline{\text { naa25 }}$ \\
\hline At3g21055 & Photosystem II subunit T(PSBTN) & $\mathrm{Wt}$, naa 25 \\
\hline At $2 \mathrm{~g} 06520$ & Photosystem II subunit X(PSBX) & $\mathrm{Wt}$, naa 25 \\
\hline Atlg44575 & Chlorophyll A-B binding family protein(NPQ4) & naa 25 \\
\hline At3g01500 & Carbonic anhydrase $1(\mathrm{CA} 1)$ & naa25 \\
\hline Atlg29910 & Chlorophyll A/B binding protein $3(\mathrm{CAB} 3)$ & naa 25 \\
\hline Atlg30380 & Photosystem I subunit K(PSAK) & naa25 \\
\hline At $2 \mathrm{~g} 30570$ & Photosystem II reaction center W(PSBW) & naa 25 \\
\hline
\end{tabular}


A

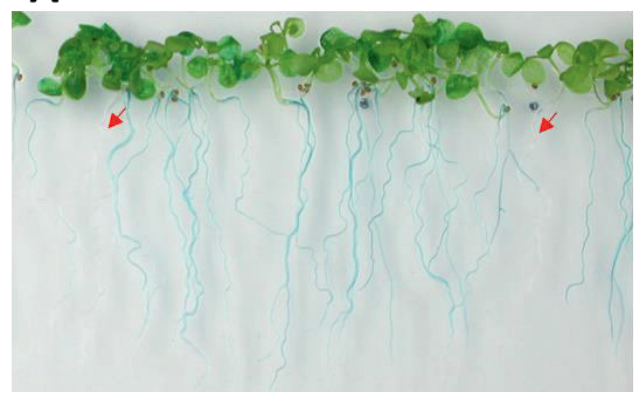

B

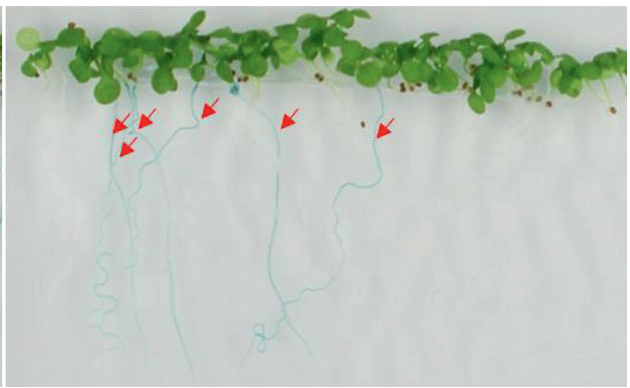

Figure S1. In vivo GUS staining assay of the pZIP4::GUS M2 mutant screen. Seedlings are grown in MS-agar plates either at (A)Zn deficiency or (B) Zn sufficiency. Arrows indicate mutants with an aberrant GUS staining phenotype, showing no staining in (A) and staining in (B).

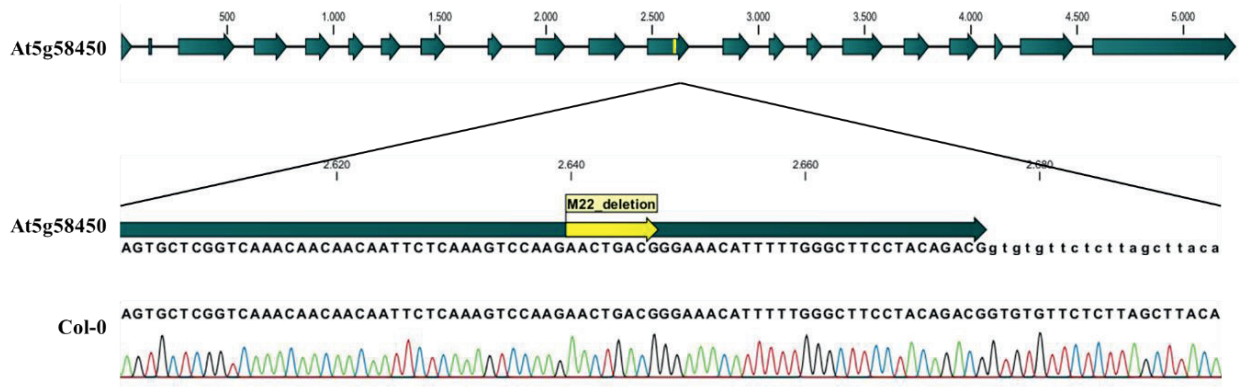

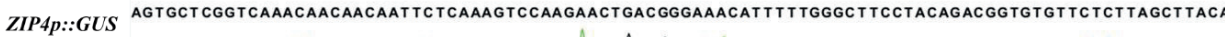

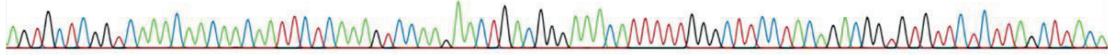

M22

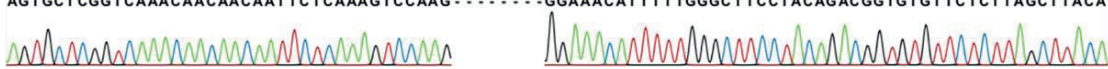

Figure S2. Sequence deletion in naa25 of the M22 mutant. NAA25 (At5g58450) sequence alignment of Col-0, pZIP4::GUS and M22 around the mutation found in M22. Numbers above the sequence represent the base number counting from the translation start. Blue arrows represent exons, lines connecting them introns, and the yellow arrow indicates the 8-bp deletion in M22. 


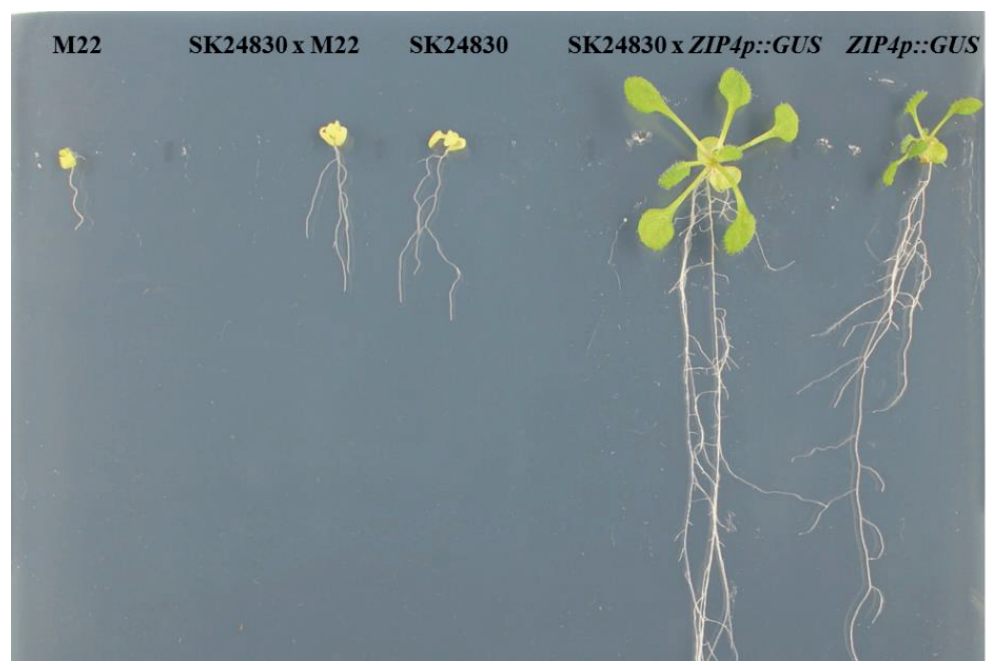

Figure S3. F1 seedlings grown under Fe deficiency. Seedlings of mutant line M22, T-DNA insertion line SK24830, pZIP4::GUS background control, F1 of M22xSK24830, and F1 of SK24830xpZIP4::GUS grown for three weeks on Fe deficiency vertical, half MS-agar plates.

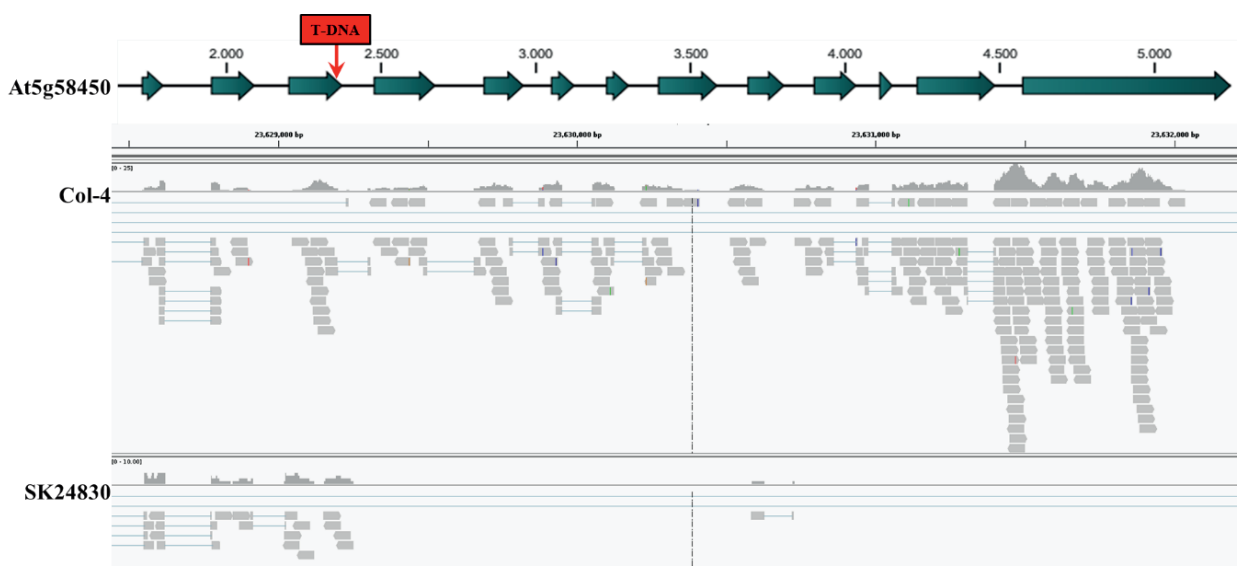

Figure S4. NAA25 (At5g58450) transcripts sequence reads from exon nine to twenty-one of Col-4 and of SK24830. Red arrow shows the position of the T-DNA insertion in SK24830. NB, there are no reads of NAA25 downstream of the T-DNA insertion, indicating the insertion to disrupt transcription of the gene. 


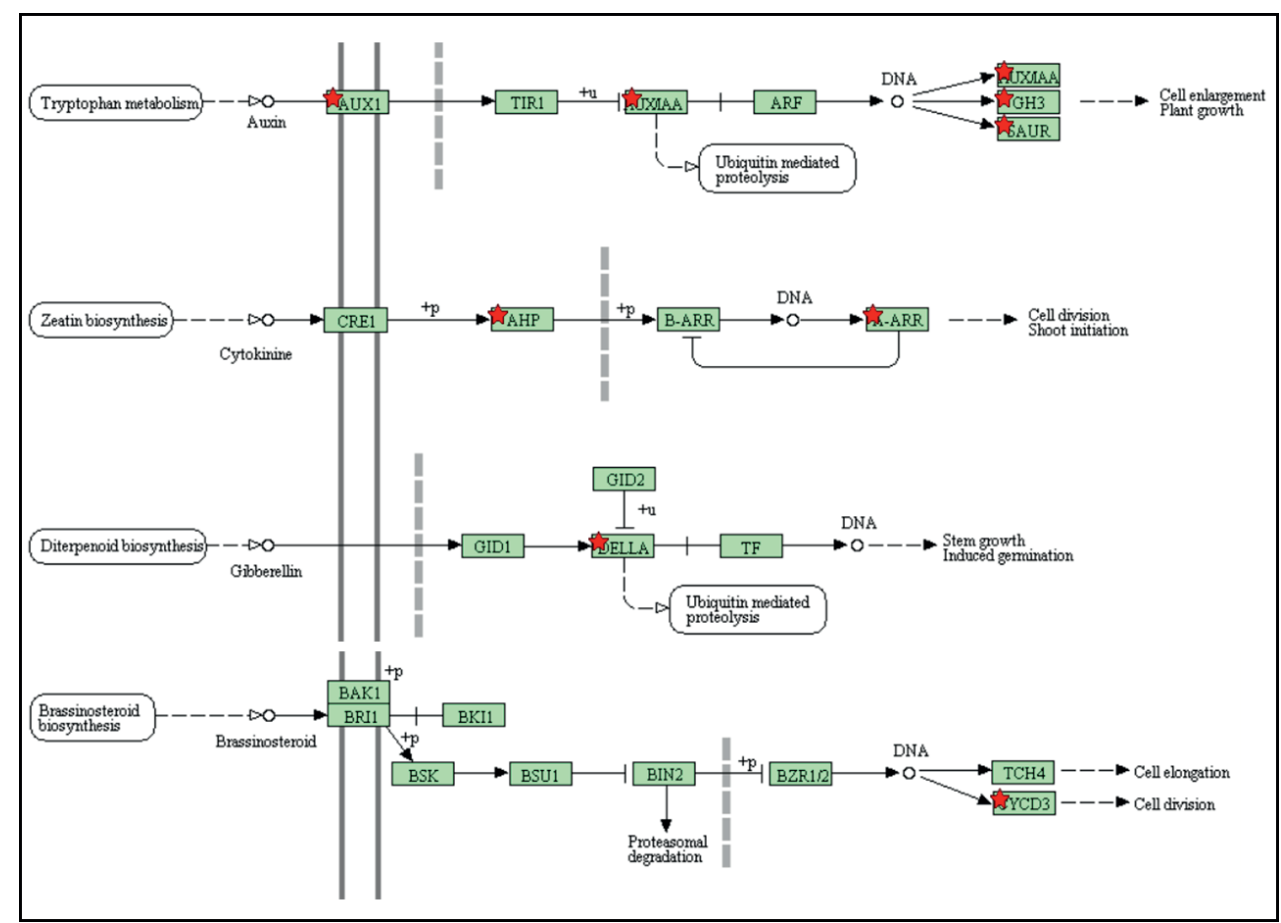

Figure S5. Plant hormone signal transduction pathway from Kyoto Encyclopedia of Genes and Genomes Pathways (KEGG). Red stars represent points where genes differentially expressed in naa25 play a role. Generated through DAVID Bioinformatics Resources 6.8 (david.ncifcrf.gov/). 


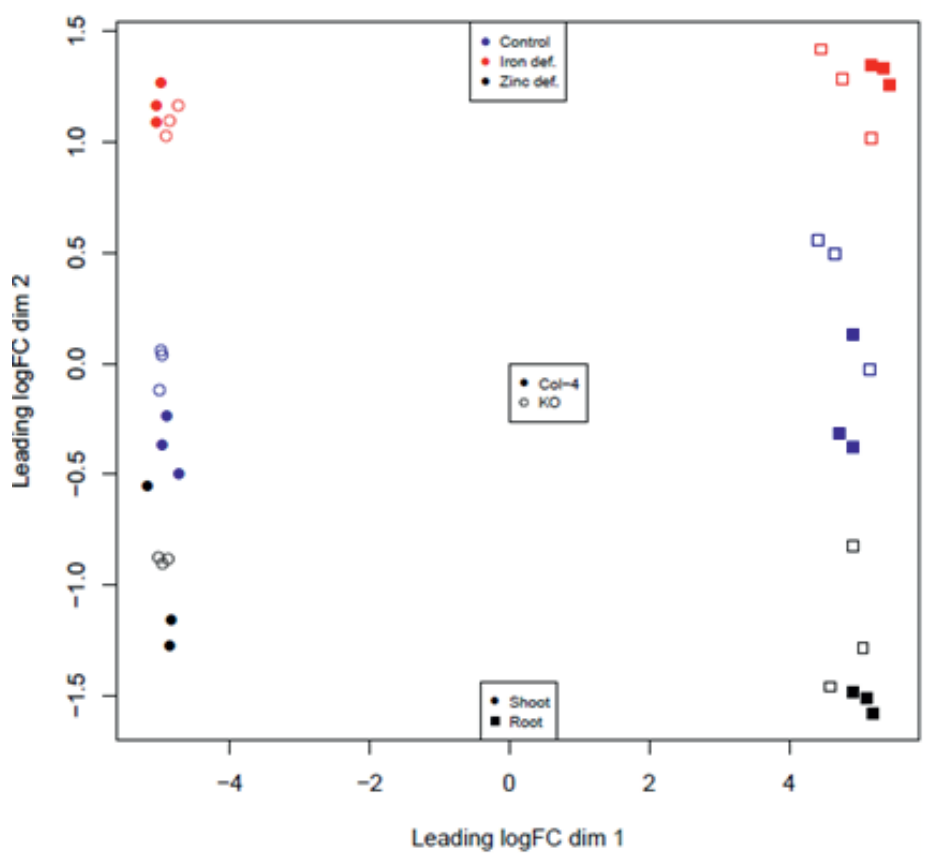

Figure S6. Multidimensional scaling transcriptome data of naa25. All expressed genes in roots (squares) and shoots (circles) of the naa25 mutant (KO: unfilled) and wild type (Col-4: filled) under Fe deficiency (red), Zn deficiency (black), or control medium (blue). 

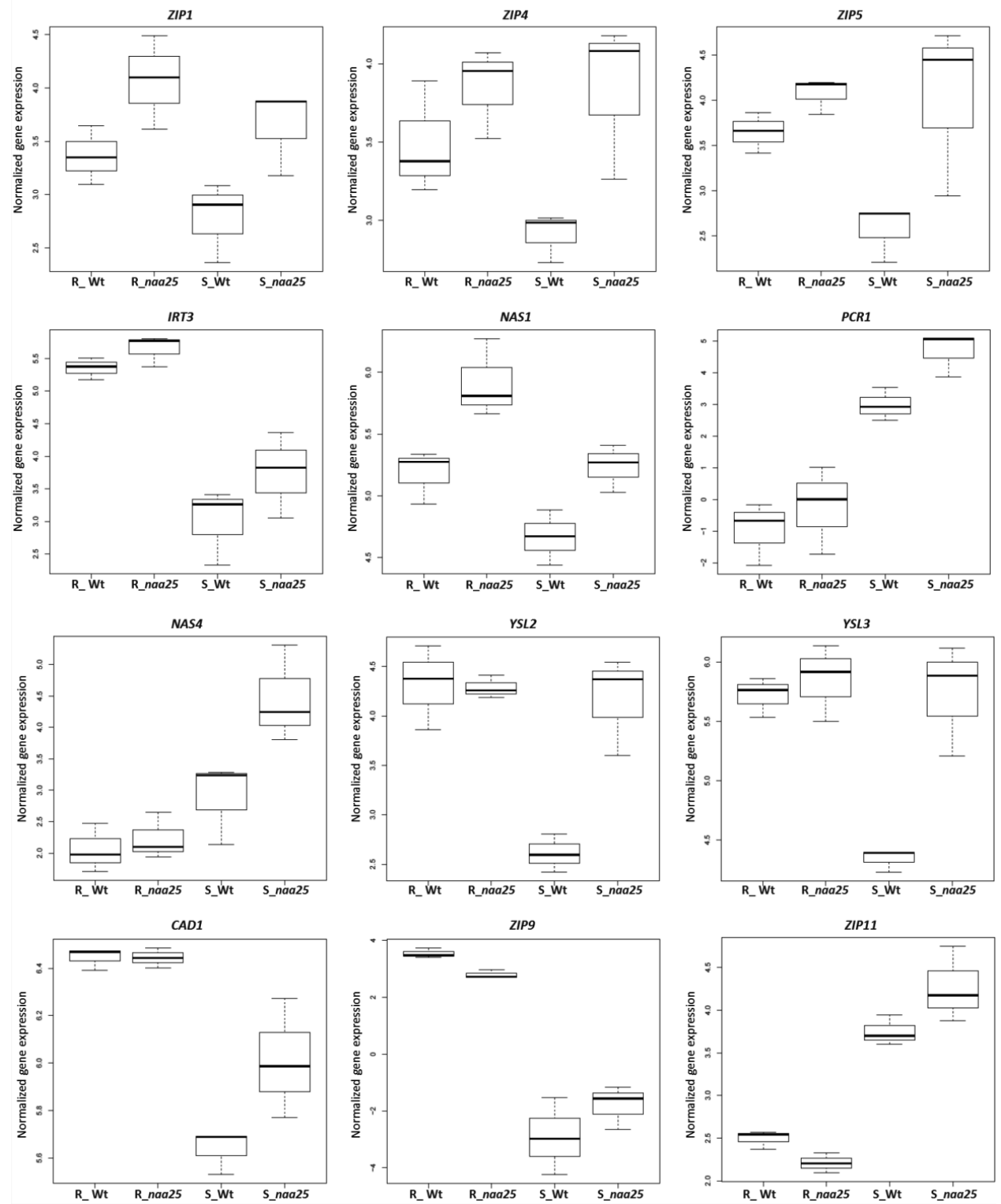

Figure S7. Normalized gene expression of Zn transporter genes. Gene expression in shoots (S_) and roots $\left(R \_\right.$) in naa 25 and wild type (Wt) under control conditions. Data from RNA-seq reads. 



\section{Chapter 4}

\section{Exploring Arabidopsis thaliana natural genetic variation to identify genes underlying the $\mathrm{Zn}$ deficiency response}

Valeria Ochoa Tufiño ${ }^{1,3}$, Ana Carolina Campos ${ }^{1,4}$, Joost van den Heuvel ${ }^{1}$, Jeroen Wolfkamp ${ }^{1}$, John Danku², David E. Salt ${ }^{2}$ \& Mark G.M. Aarts ${ }^{1}$

1) Laboratory of Genetics, Wageningen University, Wageningen, the Netherlands

2) School of Biosciences, University of Nottingham, Sutton Bonington, England, United Kingdom

3) Current affiliation: Departamento de Ciencias de la Vida, Universidad de las Fuerzas Armadas ESPE, Sangolquí, Ecuador

4) Current affiliation: Dümmen Orange, Leiderdorp, The Netherlands

In preparation for submission 


\begin{abstract}
Zn deficiency is a common micronutrient deficiency, affecting yield and nutritional quality of crops around the world. Selection due to environmental conditions and genetic drift promote genetic variation within a species, which can be used to identify the genetic factors contributing to natural variation for a trait. We used a set of 350 natural accessions of Arabidopsis thaliana (Arabidopsis) to perform a Genome-Wide Association Study (GWAS) to identify candidate genes underlying natural variation plant ionome profile upon $\mathrm{Zn}$ deficiency. $\mathrm{Zn}$ deficiency leads to an increased concentration of most of the elements analysed. Ionome profiles have been used to identify genomic regions associated with variation in $\mathrm{Cu}, \mathrm{Fe}, \mathrm{Mn}, \mathrm{Mo}$, and $\mathrm{Zn}$ concentration in response to $\mathrm{Zn}$ deficiency. Upon screening T-DNA insertion mutant lines for candidate genes, seven genes are confirmed to be involved in the $\mathrm{Zn}$ deficiency response, affecting the shoot concentrations of $\mathrm{Cu}, \mathrm{Fe}, \mathrm{Mn}, \mathrm{Mo}$, or $\mathrm{Zn}$. These genes are involved in microtubules organization during cell division, defence mechanisms against pathogens, circadian clock, $\mathrm{Fe}$ transport, Fe storage and phosphorylation of carbohydrates. Thus, genes with diverse functions play a role in the plant response to $\mathrm{Zn}$ deficiency by a direct or mediated influence on the ionome homeostasis.
\end{abstract}




\section{Introduction}

Natural genetic variation describes the phenotypic differences within a species due to genetic differences, within or between natural populations, as a result of genetic drift or selection pressure of specific environments. Arabidopsis thaliana (Arabidopsis) occupies habitats like sandy areas (beaches and river banks), roadsides, rocky mountains in a wide range of climates, which makes it a subject of wide variety of selection factors (Al-Shehbaz and O'Kane, 2002; Koornneef et al., 2004; Alonso-Blanco et al., 2009; Horton et al., 2012). Local adaptation of Arabidopsis populations, meaning local enrichment for alleles that provide optimal fitness to specific conditions in the area, can be found (Baxter et al., 2010; Fournier-Level et al., 2011).

The concentrations of elements in plant organs, also known at the ionome, is a complex of traits for which extensive genetic variation is found among Arabidopsis accessions (Buescher et al., 2010; Chao et al., 2014). The ionome can be affected by the environment and developmental stage, and it depends on several plant processes ranging from uptake, translocation, and storage. The regulation of maintaining a stable ionome is known as mineral homeostasis. Often, the mineral homeostasis of one element is affected by the concentration of others, and alteration of the concentration of one element often has consequences for the concentrations of several other elements, reflecting the complex physiological responses on changes in nutrient supply (Baxter et al., 2008; Buescher et al., 2010; Baxter et al., 2012).

The elemental concentrations of the soil have a strong effect on the plant ionome and the physiological state of the plant (Marschner and Marschner, 2012). Mineral supply can range from deficient to toxic levels for essential nutrients, while non-essential elements only have an effect when supplied at toxic levels. Among micronutrients, $\mathrm{Zn}$ deficiency is the most widespread in the world. $49 \%$ of the agricultural soils of the world are deficient in Zn. Crops affected by Zn deficiency produce a lower yield and reduced nutritional quality (Graham, 2008). Zn deficiency in soil often leads to $\mathrm{Zn}$ deficiency in humans, which is a widespread problem in most of the developing world (Alloway, 2009). Zn deficiency has serious implications for human health, leading to a decrease in learning capability (IQ) and growth retardation in developing children, while it also affects the immune system, and delays wound healing (Evans, 1986; Graham, 2008; Ackland and Michalczyk, 2016). The World Health Organisation (WHO) estimates that around three billion people suffer from some degree of $\mathrm{Zn}$ deficiency, making it a major public health problem. Better knowledge on the plant $\mathrm{Zn}$ 
homeostasis network can improve the selection for, or genetic engineering of, new crop varieties, which are more efficient with low $\mathrm{Zn}$ availability from poor $\mathrm{Zn}$ soils, and are better in the partitioning of $\mathrm{Zn}$ into harvested plant parts (Ghandilyan et al., 2006; Cakmak, 2008).

Increasing our knowledge on the natural variation for the Arabidopsis ionome is important to further understand the genetics and physiology of mineral homeostasis in plants (Salt et al., 2008; Alonso-Blanco et al., 2009). Genome-Wide Association Studies (GWAS) provide a powerful tool to identify genes and variant alleles that relate to genetic variation of a trait (Korte and Farlow, 2013), by associating genotyped markers (mainly SNPs, Single Nucleotide Polymorphisms) with their contribution to a given trait across a large set of individuals. These associated SNPs are easily identified as long as the alleles they are closely linked to are sufficiently frequent in the population and contribute sufficiently to the trait variation (Korte and Farlow, 2013). GWAS thus shows the best results for traits affected by a small number of loci with large effect sizes, with a few loci explaining most of the phenotypic variance (Atwell et al., 2010). GWAS already detected allelic variation on ionome composition, identifying relevant genes for the concentration of $\mathrm{Na}$, the HIGH-AFFINITY K+ TRANSPORTER 1 (HKT1.1) gene, encoding a Na transporter (Baxter et al., 2010); for as, the HIGH ARSENIC CONTENT 1 (HAC1) gene, encoding an arsenate reductase (Chao et al., 2014); and Cd, the HEAVY-METAL ATPASE 3 (HMA3) gene, encoding a Cd transporter (Chao et al., 2012).

In this study, we used GWAS analysis of the root and shoot ionomes of 350 natural accessions of Arabidopsis ionomics to explore the genetic variation in $\mathrm{Zn}$ deficiency response. We observed that most of the element concentrations increased significantly upon $\mathrm{Zn}$ deficiency when compared to a $\mathrm{Zn}$ sufficiency control treatment. We also identified several candidate genes that are potentially involved in Arabidopsis ionome homeostasis. Some of these were validated through the phenotypic analysis of T-DNA insertion lines in their response to $\mathrm{Zn}$ deficiency.

\section{Materials and Methods}

\section{Plant material and growth conditions}

A set of 350 natural accessions of Arabidopsis (Li et al., 2010) was used in this study. Seeds were surface-sterilized using chlorine vapor-phase (Clough and Bent, 1998). Seeds were sown on $0.8 \mathrm{ml}$ bottomless tubes filled with $0.55 \%$ agar-solidified modified half-strength Hoagland 
nutrient solution at pH 5.8 (Schat et al., 1996). Prior to germination, seeds were stratified for three days at $4{ }^{\circ} \mathrm{C}$ in the dark. Upon germination, plants were hydroponically grown in the same nutrient solution in a climate-controlled growth chamber set at 70\% humidity, a 12/12 $\mathrm{h}$ light/dark cycle, and $20 / 15^{\circ} \mathrm{C}$ day/night temperatures. Plants grew in a fully supplemented medium (containing $2 \mu \mathrm{M} \mathrm{ZnSO}_{4}$ ) for two weeks. Thereafter, two $\mathrm{Zn}$ treatments were applied, continued growth on $2 \mu \mathrm{M} \mathrm{ZnSO}_{4}$, for $\mathrm{Zn}$ sufficiency, or growth on medium containing only $0.05 \mu \mathrm{M} \mathrm{ZnSO}_{4}$, imposing a mild Zn deficiency (Campos et al., 2017). Plants grew in trays with the capacity for 70 plants. In each treatment, two replicates per genotype were grown.

Arabidopsis T-DNA insertion lines of candidate genes were ordered from the Nottingham Arabidopsis Stock Centre (NASC; www.arabidopsis.info/) (Supplemental Table S1). To multiply and genotype these lines, seeds were stratified for three days at $4^{\circ} \mathrm{C}$ in the dark, and then transferred to grow on nutrient-supplied rockwool in a greenhouse set at $70 \%$ humidity, $16 / 8 \mathrm{~h}$ light/dark cycle, and $20 / 18^{\circ} \mathrm{C}$ day/night temperatures. T-DNA insertion lines were genotyped to select or confirm homozygote plants. Primers for T-DNA insertion lines genotyping were designed using the website of the Salk Institute (www.signal.salk.edu/tdnaprimers.2.html). Confirmed homozygous T-DNA insertion mutants were grown hydroponically as described above, with the only difference that no $\mathrm{Zn}$ was added in the medium for the $\mathrm{Zn}$ deficiency treatment.

\section{Ionome analyses}

Shoot and roots of Arabidopsis plants were collected four weeks after germination. Roots and two young but fully-expanded leaves were washed with 1 mM EDTA (pH 8) and MQ water. Plant material was collected in paper bags and dried for 3 days at $60^{\circ} \mathrm{C}$. Element concentrations (ionome composition) was determined by ICP-MS as described by Baxter et al. (2008).

\section{Statistical analyses}

Ionome data from natural accessions was $\log 10$-transformed. Thereafter, we initially perform a principal component analyses using Pearson correlations and two-way ANOVA scripts within R package. Software used for GWAS was written in pure R code (Script in Supplemental data file 1) (Kruijer et al., 2015). For each element, two traits were used. first, the concentration of each element in each biological repetition within treatments, and second the residuals of the means. The residuals were calculated from the regression of each element concentration in plants grown under $\mathrm{Zn}$ deficiency and the concentration in plants grown with sufficient $\mathrm{Zn}$ 
supply, using Genstat (18th edition) (VSN International; www.vsni.co.uk/software/genstat/). To ensure that the data were normally distributed, they were $\log 10$-transformed. For most traits, the strict Bonferroni correction (Bonferroni, 1935) threshold was not met for any locus, which is why a second, arbitrary threshold of significant loci of $-\log 10(\mathrm{p}) \geq 4$ was used, which had to be met by several closely linked SNPs. Candidate genes were selected based on their Linkage Disequilibrium (LD) with the associated SNPs with a cut-off of 0.4, the presence of nonsynonymous SNPs in the coding region (Weigel and Mott, 2009), a predicted or putative function related to mineral homeostasis, and gene expression upon $\mathrm{Zn}$ deficiency (Campos, 2015).

Ionome data obtained from the first test of T-DNA insertion lines of the candidate genes was ln-transformed to generate Biplots (Gabriel, 1971) using Genstat (18th edition) (VSN International; www.vsni.co.uk/software/genstat/). For each element, biplots graphs were generated for four variables (element concentrations in shoots and roots of plants grown under Zn sufficiency and deficiency).

For T-DNA insertion lines test, we fitted mixed effects models with a Gaussian error distribution (lmer function, lme4 package). Treatment and genotype were used as fixed variables, trays as random variables. We fitted first a full factorial model, a model with the two main effects and separately two models with one main effect. With the obtained p-values for the main effects and interaction terms, a likelihood ratio test (Chi-square test using the ANOVA function) was performed between the models with one and two main effects (for main effect significance) and between the full factorial model and the model with two main effects (for the interaction term). All p-values were corrected for multiple testing (p.adjust function) using the Benjamini and Hochberg (1995) method. An alpha value of 0.05 was used as significant for the False Discovery Rate (FDR)-transformed p-values. To ensure that the residuals were normally distributed and that their variances were not heterogeneous between treatment levels, we square-root-transformed the biomass data and ln-transformed the ionome data. To visualize the differences between treatment levels, heatmaps were produced (heatmap function from the heatmap package). Fold changes were calculated of element concentrations as follows for genotype (Y $\left.\mathrm{Y}_{\mathrm{TDNA}, \mathrm{DEF}}+\mathrm{Y}_{\mathrm{TDNA}, \mathrm{SUF}}\right) /\left(\mathrm{Y}_{\mathrm{WT}, \mathrm{DEF}}+\mathrm{Y}_{\mathrm{WT}, \mathrm{SUF}}\right)$ and for interaction (Y $\mathrm{TDNA,DEF} /$ $\left.\mathrm{Y}_{\mathrm{WT}, \mathrm{DEF}}\right) /\left(\mathrm{Y}_{\mathrm{TDNA}, \mathrm{SUF}} / \mathrm{Y}_{\mathrm{WT}, \mathrm{SUF}}\right)$ where $\mathrm{Y}$ stands for the mean of a trait value for a specific combination of a genotype and treatment value (WT = control genotype, TDNA = T-DNA line genotype, $\mathrm{DEF}=$ deficiency treatment, $\mathrm{SUF}=$ sufficiency treatment). For the calculation of these 
fold change values, also the biomass data were square-root-transformed and the ionome data were ln-transformed.

\section{Results}

\section{Zinc deficiency effects on the Arabidopsis ionome}

In this study, we grew a set of 350 natural accessions of Arabidopsis on hydroponic medium with sufficient or mild deficient $\mathrm{Zn}$ supply. Root and shoot ionome profiles were determined by ICP-MS for B, Ca, Co, Cu, Fe, K, Mg, Mn, Mo, Na, P, S, and Zn. Most of the variation of the ionome of the natural accession was explained by two principal components (PCs) in a PC analysis (PCA) (Figure 1 and Supplemental Figure S1). These two PCs separated the data according to the tissue (PC1: shoot or root) and to the treatment (PC2: sufficiency or deficiency). The elements with the highest relative contribution to PC1 were $\mathrm{Zn}>\mathrm{Ca}>\mathrm{Fe}>\mathrm{Na}>\mathrm{Cu}$ and for $\mathrm{PC} 2$ were $\mathrm{Zn}>\mathrm{Ca}>\mathrm{Na}>\mathrm{Mg}>\mathrm{Mo}$. Elements that accumulated more in roots than in shoots were $\mathrm{Co}, \mathrm{Cu}, \mathrm{Fe}, \mathrm{Mo}, \mathrm{Na}$, and $\mathrm{Zn}$. Elements that accumulated more in shoots than in roots were $\mathrm{Ca}$ and $\mathrm{Mg}$. Elements $\mathrm{B}, \mathrm{K}, \mathrm{Mn}, \mathrm{P}$, and $\mathrm{S}$ are found at similar concentrations in roots and shoots (Supplemental Figure S2).

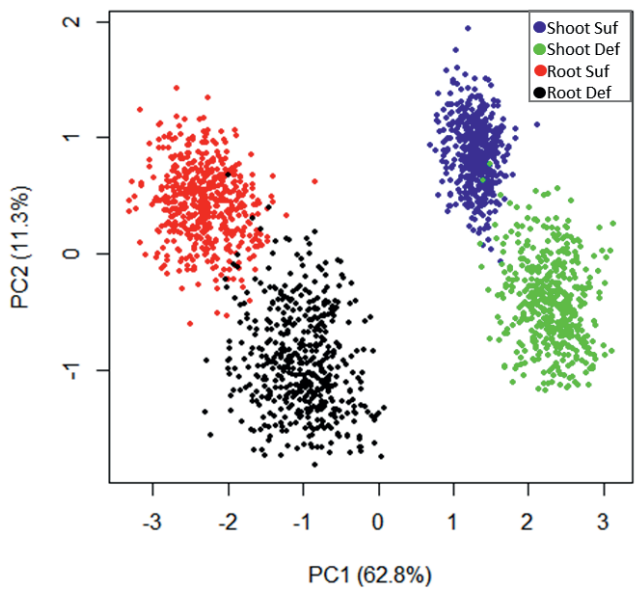

Figure 1. Principal component analyses of 13 essential elements ( $B, C a, C o, C u, F e, K, M g, M n, M o$, $N a, P, S$ and $\mathrm{Zn})$ in 350 natural accessions of Arabidopsis. Data correspond to the ionome of shoots (blue dots) and roots (red dots) of plants grown hydroponically in Zn sufficiency and shoots (green dots) and roots (black dots) of plants grown hydroponically in Zn deficiency. For the elements with the highest relative contribution in: $\mathrm{PCl}$ the positive loadings were for $\mathrm{Ca}$ and negative ones for $\mathrm{Zn}, \mathrm{Fe}, \mathrm{Na}, \mathrm{Cu}$; and PC2 the positive loadings were for $\mathrm{Zn}, \mathrm{Ca}$, and $\mathrm{Mg}$ and negative loadings for $\mathrm{Na}$ and $\mathrm{Mo}$. 
Due to the different ionome compositions in roots and shoots, we analysed both parts independently. In shoots, most of the element concentrations correlated positively with each other over accessions, when comparing the two treatments, with the exception of B. In roots the number of negative correlations was higher than in shoots, with the Ca concentrations mostly negatively correlated with the other element concentrations (Figure 2).

Shoot Sufficiency

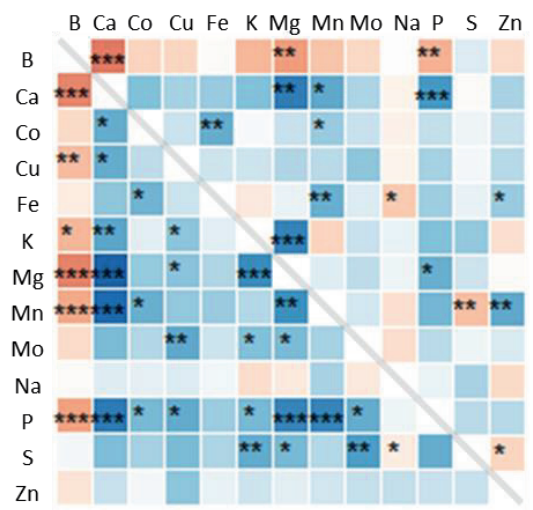

Shoot Deficiency
Root Sufficiency

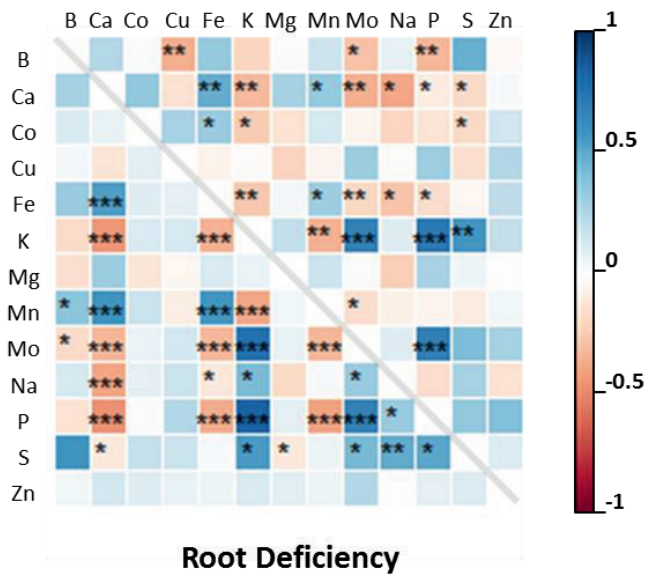

Figure 2. Pearson correlations of ionome profiles in 350 natural accessions of Arabidopsis. Element concentration in shoots and roots of plants grown hydroponically in Zn sufficiency or deficiency are compared. Elements are in alphabetic order. Positive and negative correlations are indicated by colours as shown in the sidebar. The significance level is represented with $*_{p}<0.05, * *_{p}<0.01, * * * p<0.001$.

The $\mathrm{Zn}$ deficiency treatment affects the direction of just a few correlations among elements. Negative correlations that became positive due to $\mathrm{Zn}$ deficiency were Fe-Na, Mn-S, and Zn-S in shoots and $\mathrm{B}-\mathrm{Cu}, \mathrm{Co}-\mathrm{K}, \mathrm{Co}-\mathrm{S}, \mathrm{Mg}-\mathrm{Mn}$, and Na-P in roots. Positive correlations that became negative due to $\mathrm{Zn}$ deficiency were $\mathrm{S}-\mathrm{Na}$ in shoots and $\mathrm{S}-\mathrm{Mg}$ in roots. Overall, the shoot to root concentration ratios did not change much due to $\mathrm{Zn}$ deficiency. The correlation coefficient for element concentrations in shoot sufficiency and shoot deficiency was 0.78 and for roots, it was $0.80(p<0.001)$. While correlations remain largely similar, the concentrations of most elements were significantly affected by Zn deficiency. In shoots, Zn deficiency increased the B, Ca, Co, $\mathrm{Fe}, \mathrm{Mg}, \mathrm{Mn}, \mathrm{Mo}, \mathrm{P}$, and $\mathrm{S}$ concentrations and decreased the $\mathrm{Cu}$ and $\mathrm{Na}$ concentrations (Table 1). In roots, $\mathrm{Zn}$ deficiency increased the $\mathrm{B}, \mathrm{Ca}, \mathrm{K}, \mathrm{Na}$, and $\mathrm{S}$ concentrations and decreased the $\mathrm{Cu}, \mathrm{Fe}, \mathrm{Mg}$, and $\mathrm{P}$ concentrations (Table 1). The concentration of $\mathrm{K}$ in shoots and the 
concentrations of $\mathrm{Co}, \mathrm{Mn}$, and $\mathrm{Mo}$ in roots were not altered due to $\mathrm{Zn}$ deficiency. The mean $\mathrm{Zn}$ concentration decreased in shoots from $81 \mathrm{ppm}$ to $14 \mathrm{ppm}$ in $\mathrm{Zn}$ deficiency and in roots from $288 \mathrm{ppm}$ to $65 \mathrm{ppm}$, which is translated to $82 \%$ reduction in shoots and $77 \%$ reduction in roots. The element with the strongest changes after $\mathrm{Zn}$ was $\mathrm{Fe}$, with a $35 \%$ increase in shoots and $67 \%$ reduction in roots due to $\mathrm{Zn}$ deficiency (Supplemental Figure S3).

Table1. Ionome profiles of shoots and roots of 350 natural Arabidopsis accessions grown hydroponically in Zn sufficient and Zn deficient conditions. Data include mean, standard deviation, Coefficient of Variation $(\mathrm{CV})$ and broad sense heritability $\left(H^{2}\right)$. ANOVA $p$ values are represented: ${ }^{*} p<0.05,{ }^{*} p<0.001,{ }^{* * *}<<0.001$. Shadow indicates interaction of genotype (Gen) $x$ treatment (Trt).

\begin{tabular}{|c|c|c|c|c|c|c|c|c|c|c|}
\hline \multirow[b]{3}{*}{ Shoot } & \multicolumn{4}{|c|}{ Control } & \multicolumn{4}{|c|}{ Deficiency } & \multicolumn{2}{|c|}{ ANOVA } \\
\hline & $\begin{array}{l}\text { Mean } \\
\text { [ppm] }\end{array}$ & Std dev & $\begin{array}{l}\text { C.V } \\
{[\%]}\end{array}$ & $\begin{array}{c}\mathbf{H}^{2} \\
{[\%]}\end{array}$ & $\begin{array}{l}\text { Mean } \\
\text { [ppm] }\end{array}$ & Std dev & $\begin{array}{l}\text { C.V } \\
{[\%]}\end{array}$ & $\begin{array}{c}\mathbf{H}^{2} \\
{[\%]}\end{array}$ & Trt & Acc \\
\hline & & & & & & & & & & \\
\hline B & 148.7 & 60.66 & 41 & 41 & 185.6 & 88.8 & 48 & 49 & $* * *$ & \\
\hline $\mathrm{Ca}$ & 36319 & 7280 & 20 & 43 & 46230 & 14478 & 31 & 42 & $* * *$ & \\
\hline Co & 0.15 & 0.03 & 20 & 46 & 0.16 & 0.03 & 21 & 42 & $* * *$ & \\
\hline $\mathrm{Cu}$ & 3.048 & 0.615 & 20 & 45 & 2.475 & 0.596 & 24 & 53 & $* * *$ & $* * *$ \\
\hline $\mathrm{Fe}$ & 113.8 & 18.13 & 16 & 41 & 153.1 & 38.18 & 25 & 45 & $* * *$ & \\
\hline $\mathbf{K}$ & 28248 & 7920 & 28 & 48 & 28123 & 9384 & 33 & 48 & & $*$ \\
\hline Mg & 3577 & 698.9 & 20 & 48 & 4165 & 1038 & 25 & 51 & $* * *$ & $*$ \\
\hline Mn & 66.3 & 18.05 & 27 & 47 & 87.61 & 36.48 & 42 & 49 & $* * *$ & $* * *$ \\
\hline Mo & 16.84 & 6.14 & 36 & 65 & 22.04 & 8.58 & 39 & 61 & $* * *$ & $* * *$ \\
\hline $\mathrm{Na}$ & 622.1 & 195.7 & 31 & 39 & 545 & 257.7 & 47 & 45 & $* * *$ & \\
\hline $\mathbf{P}$ & 5923 & 1679 & 28 & 44 & 6479 & 1674 & 26 & 54 & $* * *$ & $* *$ \\
\hline $\mathbf{S}$ & 9386 & 1587 & 17 & 42 & 10077 & 2142 & 21 & 47 & $* * *$ & \\
\hline $\mathrm{Zn}$ & 81.58 & 22.33 & 27 & 50 & 14.48 & 6.37 & 44 & 44 & $* * *$ & \\
\hline Root & & & & & & & & & & \\
\hline B & 142 & 69 & 49 & 40 & 191.4 & 87.64 & 46 & 45 & $* * *$ & \\
\hline $\mathrm{Ca}$ & 6371 & 1459 & 23 & 44 & 7408 & 1940 & 26 & 59 & $* * *$ & $* * *$ \\
\hline Co & 0.29 & 0.13 & 44 & 44 & 0.38 & 0.46 & 120 & 43 & & \\
\hline $\mathrm{Cu}$ & 10.95 & 3.35 & 31 & 50 & 7.93 & 2.01 & 25 & 53 & $* * *$ & $* * * *$ \\
\hline $\mathrm{Fe}$ & 972.5 & 339.6 & 35 & 47 & 318.9 & 117.6 & 37 & 57 & $* * *$ & $* * *$ \\
\hline $\mathbf{K}$ & 25584 & 9796 & 38 & 50 & 25821 & 14963 & 58 & 61 & $* * *$ & $* * *$ \\
\hline Mg & 1583 & 269.3 & 17 & 56 & 1358 & 291.9 & 21 & 56 & $* * *$ & $* * *$ \\
\hline Mn & 69.89 & 33.47 & 48 & 48 & 65.62 & 23.88 & 36 & 48 & & $* *$ \\
\hline Mo & 55.49 & 32.37 & 58 & 57 & 54.06 & 33.79 & 63 & 56 & & $* * *$ \\
\hline $\mathrm{Na}$ & 1820 & 570 & 31 & 38 & 2253 & 867 & 38 & 55 & $* * *$ & $* * *$ \\
\hline $\mathbf{P}$ & 7919 & 1863 & 24 & 54 & 6537 & 2462 & 38 & 58 & $* * *$ & * \\
\hline $\mathbf{S}$ & 7919 & 2114 & 27 & 43 & 9198 & 3060 & 33 & 56 & $* * *$ & $* *$ \\
\hline$Z_{n}$ & 288.1 & 106.6 & 37 & 53 & 65.26 & 39.21 & 60 & 57 & $* * *$ & $* * *$ \\
\hline
\end{tabular}




\section{Genome-Wide Association Mapping}

The processes that influence ionome homeostasis in plants are complex and likely to be driven by a large number of loci with small effects. To expand our knowledge of the genetic architecture underlying ionome homeostasis, with a focus on $\mathrm{Zn}$ deficiency tolerance, we performed a GWAS. We narrowed the analyses to the micronutrients with concentrations that were positively correlated with the $\mathrm{Zn}$ concentration, irrespective of the treatment and plant part, which meant using the concentration of $\mathrm{Co}, \mathrm{Cu}, \mathrm{Fe}, \mathrm{Mn}, \mathrm{Mo}$, and $\mathrm{Zn}$ of the 350 natural accessions of Arabidopsis. In addition to each element concentration, we used the residuals, of the regression of the element concentrations means, of plants grown under $\mathrm{Zn}$ deficiency on plants grown under sufficient Zn supply.

Among the Arabidopsis accessions, large variation in ionome profile was observed, especially in roots. We expressed the relative variability of each element in terms of Coefficient of Variation (CV), which is the ratio of the standard deviation to the mean. This is useful to compare the variation of elements with highly different means. The variation was higher in roots than in shoots and in deficiency than in sufficiency (Table 1). In shoots, the elements with the highest variation were $\mathrm{B}>\mathrm{Na}>\mathrm{Mo}>\mathrm{Zn}>\mathrm{Mn}$ and in roots $\mathrm{Co}>\mathrm{Mo}>\mathrm{Zn}>\mathrm{K}>\mathrm{Mn}$. Broad-sense heritability was calculated for all element concentrations, as the ratio between estimated genetic variance and total phenotypic variance, which itself is composed of genetic variance and nongenetics, or environmental, variance. This is a measure of how much of the phenotypic variation is due to genetic variation. Heritability ranged from $39 \%$ to $66 \%$ depending on the element, plant part, and treatment. In shoots, the elements with the highest heritably were $\mathrm{Mo}>\mathrm{Mg}>\mathrm{Cu}=\mathrm{P}>\mathrm{Mn}$ and in roots $\mathrm{Mo}=\mathrm{P}>\mathrm{Zn}=\mathrm{Mg}=\mathrm{K}$. With the exception of $\mathrm{B}$ and $\mathrm{Co}$, all elements showed a significant effect of genotype on element concentrations (Table 1), which also indicates the genetic control of elements accumulation in the plant. For Ca, Fe, K, Mo, P, and $\mathrm{Zn}$ concentration in roots, a significant genotype $\mathrm{x}$ treatment interaction was found (Table 1).

For all these element concentrations or their residuals, one or more associated SNP markers were found for at least one locus (Supplemental data file 2). Based on the criteria mentioned in the materials and methods section, we selected the loci more likely to contain genes involved in the ionome homeostasis (Figure 3). For Co, one locus was selected, which was detected in roots either using Co concentration or residuals. For $\mathrm{Cu}$, five loci were selected: three associated with root concentration and two associated with the residuals for root. For Fe, we selected four 
loci: one associated with Fe root concentration and Mn root concentration; one associated with Fe shoot concentration; and two associated with Fe shoot concentration and residuals. For Mn, we selected six loci: two associated with Mn root residuals and four associated with Mn shoot concentration. For Mo, we selected two loci associated with Mo shoot concentration, the loci (in chromosome two) was also associated with Mo shoot residuals and Mo root concentration. For Zn, we selected four loci: two associated with $\mathrm{Zn}$ shoot concentration; one associated with $\mathrm{Zn}$ shoot residuals; and one associated with $\mathrm{Zn}$ shoot residuals. The average length of each region covered by the associated SNPs was of $39 \mathrm{~Kb}$. Possible candidate genes residing at those loci were selected, based in the level of significance of associated SNPs, and consistency due to the presence of several associated SNPs on LD.

\section{Candidate genes with previously described roles in ionome homeostasis}

Several of the candidate genes encode for proteins with putative functions related to the ionome homeostasis. In the selected loci (Figure 3), are genes encoding for K UPTAKE PERMEASE 11 (KUP11), HEAVY METAL-ASSOCIATED ISOPRENYLATED PLANT PROTEIN 21 (HIPP21), PURPLE ACID PHOSPHATASE 26 (PAP26), and TONOPLAST INTRINSIC PROTEIN 2;3 (TIP2;3) (all for Cu); CASPARIAN STRIP MEMBRANE PROTEIN 5 (CASP5) (Fe); NATURAL RESISTANCE-ASSOCIATED MACROPHAGE PROTEIN 1 (NRAMP1) (Mn); MOT1 (Mo); and FERRITIN 4 (FER4), and HIPPS 10,11, 12, 13, 14 (Zn). Of these genes, only NRAMPI (Mn) (Cailliatte et al., 2010; Castaings et al., 2016) and MOT1 (Mo) (Tomatsu et al., 2007; Baxter et al., 2008) have previously been implicated in Mn and Mo homeostasis respectively. Thus we did not further test these two genes. The other genes are not known to control homeostasis of the element with which they are associated. Of them, KUP11 was not tested because we lacked a homozygous T-DNA insertion line for this gene. 

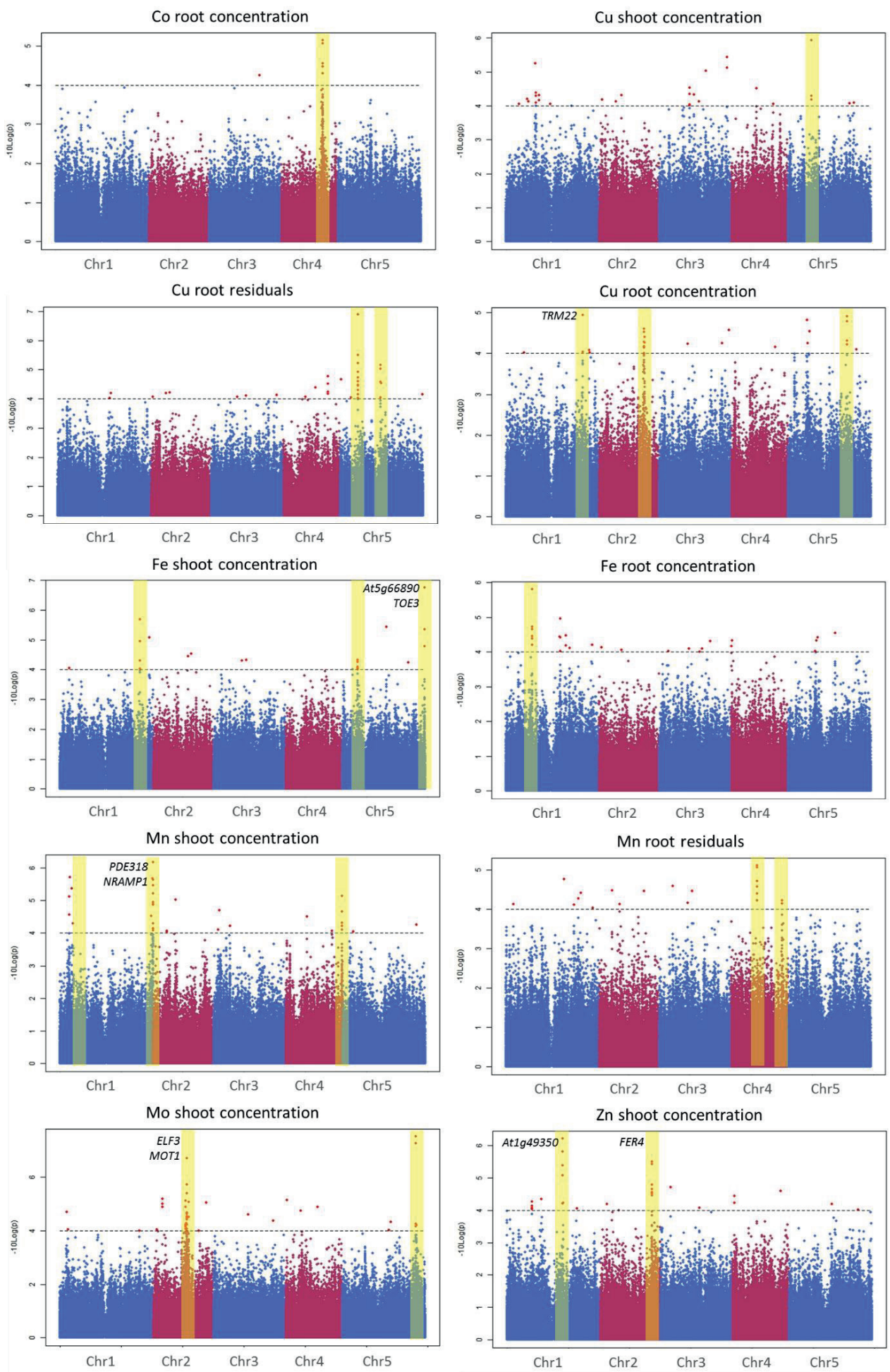

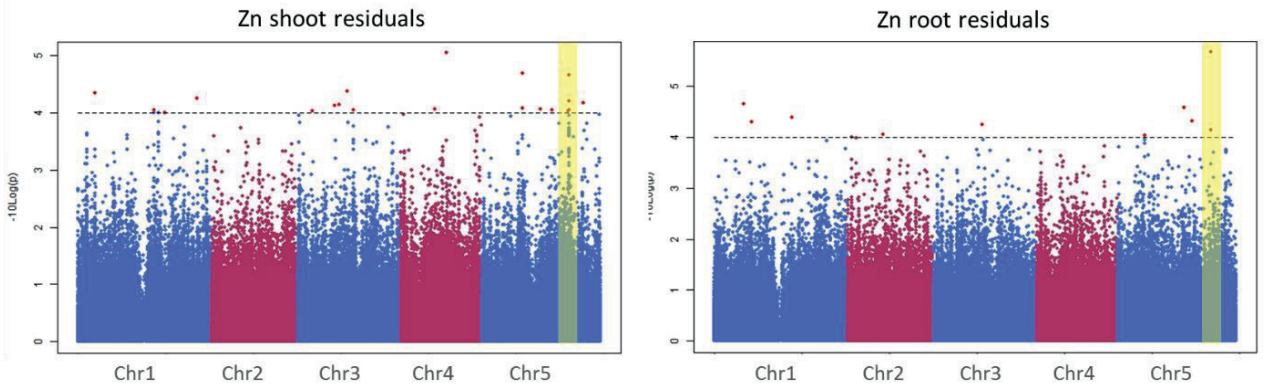

Figure 3. Manhattan plots of a GWAS based on 250k SNPS across 350 natural accessions of Arabidopsis. SNPs (blue and red dots) above the arbitrary threshold of $-\log (p)=4$ (dashed line) are considered to be associated with the trait. Yellow bars indicate the selected loci per trait, associated SNPS and genes within each selected loci are listed in Supplemental data file 2. Names of genes positively validated for the trait associated are written next to the locus they are located.

\section{Validation of novel candidate genes}

In order to evaluate the role of these candidate genes in affecting the associated element concentration in response to $\mathrm{Zn}$ deficiency, T-DNA insertion mutants were studied. Unfortunately, not all candidate genes could be evaluated, as not for all, a relevant homozygous T-DNA insertion mutant was available. A total of 38 T-DNA insertion mutants, corresponding to thirty-four genes, were examined (Table 2). These mutant lines were grown hydroponically in $\mathrm{Zn}$ sufficiency or $\mathrm{Zn}$ deficiency medium and their shoot and root ionome profiles were determined (Supplemental Figure S4). For each element, a PCA Biplot was performed (Figure 4), to identify lines with an exceptional ionome profile. In this way, eleven mutants were identified with an aberrant ionome profile. These are mutants T-082 and T-083, both mutated in TON1 RECRUITING MOTIF 22 (TRM22), associated with Cu; mutants T-104, T108, and T-113, respectively mutated in At5g66890 (encoding a leucine-rich repeat protein), TARGET OF EARLY ACTIVATION TAGGED 3 (TOE3), and CASP5, associated with Fe; mutants T-091, T-122, respectively mutated in MAJOR FACILITATOR SUPERFAMILY 1 (MSF1) and PIGMENT DEFECTIVE 318 (PDE318), associated with Mn; mutant T-128, mutated in EARLY FLOWERING 3 (ELF3), associated with Mo; and mutants T-093, T-094, T132, T-135 and T-141, respectively mutated in HIPP10, HIPP11, B-BOX DOMAIN PROTEIN 8 (BBX8), At $\lg 49350$ (encoding a pfkB-like carbohydrate kinase protein), and FER4, associated with $\mathrm{Zn}$. 
Table 2. Candidate genes identified upon GWAS of element concentrations under Zn sufficiency and Zn deficiency treatments and selected for further phenotypic analysis, and their respective T-DNA insertion mutant. For each candidate gene is detailed its locus identifier, symbol, Linkage Disequilibrium (LD) with the SNP associated, position of the SNP associated in the respective chromosome (Chr), level of significance of the association $\left(-\log _{10}(p)\right)$, and the associated element either in concentration (con) and/or residuals (res).

\begin{tabular}{|c|c|c|c|c|c|c|c|}
\hline Trait & Chr & Position & $-\log _{10}(p)$ & LD & $\begin{array}{c}\text { Locus } \\
\text { identifier }\end{array}$ & Symbol & T-DNA \\
\hline Co res, Co con root & 4 & 13503272 & 4.4 & 0.8 & At4g26850 & VTC2 & $\mathrm{T}-067$ \\
\hline Co res, Co con root & 4 & 13551025 & 4.4 & 0.8 & At4g26970 & $\mathrm{ACO} 2$ & T-068, 69 \\
\hline Co res, Co con root & 4 & 13551025 & 4.4 & 1.0 & At4g26990 & & $\mathrm{T}-070$ \\
\hline $\mathrm{Cu}$ con root & 1 & 25017792 & 4.0 & 1.0 & At1g67040 & TRM22 & $\mathrm{T}-082,83$ \\
\hline $\mathrm{Cu}$ con root & 2 & 14699246 & 4.6 & 1.0 & At $2 \mathrm{~g} 34830$ & WRKY35 & T- $-084,85$ \\
\hline $\mathrm{Cu}$ con root & 5 & 19248527 & 4.9 & 1.0 & At5g47450 & $T I P 2 ; 3$ & $\mathrm{~T}-087$ \\
\hline $\mathrm{Cu}$ res root & 5 & 5737381 & 6.9 & 1.0 & At5g17420 & CESA7 & $\mathrm{T}-073$ \\
\hline $\mathrm{Cu}$ res root & 5 & 5737381 & 6.9 & 0.4 & At5g17450 & HIPP21 & $\mathrm{T}-074$ \\
\hline $\mathrm{Cu}$ res root & 5 & 13111080 & 5.1 & 1.0 & At5g34850 & $P A P 26$ & $\mathrm{~T}-078$ \\
\hline $\mathrm{Fe}$ con, $\mathrm{Mn}$ con root & 1 & 8530152 & 5.8 & 1.0 & Atlg24120 & $A R L 1$ & T-089 \\
\hline Fe con shoot & 5 & 4966992 & 4.3 & 1.0 & At5g15290 & CASP5 & $\mathrm{T}-113$ \\
\hline Fe con, Fe res shoot & 1 & 26154033 & 5.7 & 1.0 & At1g69550 & & $\mathrm{T}-111$ \\
\hline Fe con, Fe res shoot & 1 & 26154033 & 5.7 & 0.4 & At1g69600 & ZFHDI & $\mathrm{T}-112$ \\
\hline Fe con, Fe res shoot & 5 & 26714464 & 4.4 & 1.0 & At5g66890 & & $\mathrm{T}-104$ \\
\hline Fe con, Fe res shoot & 5 & 26806136 & 5.2 & 1.0 & At5g67180 & TOE3 & $\mathrm{T}-108$ \\
\hline Mn con shoot & 1 & 4382479 & 4.3 & 0.5 & At1g12950 & RSH2 & T-119, 120 \\
\hline Mn con shoot & 1 & 30358428 & 6.2 & 1.0 & At1g80770 & PDE318 & $\mathrm{T}-122$ \\
\hline Mn con shoot & 1 & 30358428 & 6.2 & 1.0 & At1g80780 & & $\mathrm{T}-123$ \\
\hline Mn con shoot & 4 & 18442698 & 4.1 & 0.9 & At4g39700 & HIPP23 & $\mathrm{T}-126$ \\
\hline Mn con & 1 & 3917803 & 5.4 & 0.4 & At1g11670 & & $\mathrm{T}-117$ \\
\hline Mn res root & 4 & 8390639 & 5.1 & 1.0 & At4g14630 & $G L P 9$ & T-90 \\
\hline Mn res root & 4 & 16640140 & 4.1 & 0.6 & At4g34950 & $M F S I$ & T-91 \\
\hline Mo con shoot & 2 & 11032027 & 4.7 & 1.0 & At $2 \mathrm{~g} 25930$ & $E L F 3$ & $\mathrm{~T}-128$ \\
\hline Mo con shoot & 5 & 23904706 & 7.5 & 1.0 & At5g59250 & HP59 & T-129 \\
\hline Zn con shoot & 1 & 18264724 & 5.4 & 1.0 & At1g49350 & & $\mathrm{T}-135$ \\
\hline Zn con shoot & 1 & 18379245 & 4.2 & 1.0 & At1g49660 & CXE5 & $\mathrm{T}-138$ \\
\hline Zn con shoot & 2 & 16851432 & 4.5 & 0.7 & At2g40300 & FER4 & $\mathrm{T}-141$ \\
\hline Zn con shoot & 2 & 16852617 & 4.7 & 0.6 & At $2 g 40435$ & & $\mathrm{~T}-143$ \\
\hline Zn con shoot & 2 & 16896465 & 5.4 & 1.0 & At $2 \mathrm{~g} 40460$ & & $\mathrm{~T}-144$ \\
\hline $\mathrm{Zn}$ res root & 5 & 21383803 & 5.8 & 0.4 & At5g52720 & HIPPI0 & T-093 \\
\hline
\end{tabular}




\begin{tabular}{llllllll} 
Zn res root & 5 & 21383803 & 5.8 & 0.4 & At5g52730 & HIPP11 & T-094 \\
Zn res root & 5 & 21383803 & 5.8 & 0.5 & At5g52740 & HIPP12 & T-096 \\
Zn res root & 5 & 21383803 & 5.8 & 1.0 & At5g52760 & HIPP14 & T-098 \\
Zn res shoot & 5 & 19569570 & 4.0 & 0.9 & At5g48250 & BBX8 & T-132 \\
\hline
\end{tabular}

In addition to an aberrant ionome profile, these nine mutant lines showed significant changes in biomass due to the $\mathrm{Zn}$ deficiency treatment effect when compared to wild-type plants, especially mutant T-090 which is remarkable here. This line is mutated in GERMIN-LIKE PROTEIN 9 (GLP9), and showed the largest increase of all tested mutants, in both shoot and root biomass due to the $\mathrm{Zn}$ deficiency treatment, compared to its wild-type background (Supplemental Figure S5).

Based on these preliminary results, eight of the tested mutant lines were selected for confirmation of their observed Zn deficiency phenotype. These lines, (T-083, T-090, T-104, T108, T-122, T-128, T-135, and T-141) were regrown in a second phenotyping experiment, under comparable conditions (Figure 5). In this experiment, 12 replicate plants were used per mutant, which meant all biomass differences, when compared, the treatment effect between wild-type Col-0 and the mutants were significantly different (Supplemental Figure S5). Mutants T-083, T-122, T-128, T-135, and T-141 showed a significant decrease in root and shoot biomass, due to the $\mathrm{Zn}$ deficiency treatment, compared to their wild-type background. Instead, and as found in the initial experiment, mutant T-090 showed an increase in root and shoot biomass. For line $\mathrm{T}-104$, only root biomass was decreased.

Ionome concentrations were determined for shoots and roots of these mutant and wild-type plants. All mutant lines showed significant differences for at least one element when compared to their wild type (Figure 6). Six of them showed significant differences, due to genotype $\mathrm{x}$ treatment interaction, in the element to which each T-DNA line candidate gene was associated with in the GWAS (Figure 6). Those lines were: T-083 associated with $\mathrm{Cu}$ in roots, T-104 and T-108 associated with Fe in shoots, T-128 associated with Mo in shoots and roots, and T-135 and T-141 associated with $\mathrm{Zn}$ in shoots (Supplemental Figure S6). Five of the eight T-DNA lines also showed a significant decrease in $\mathrm{Zn}$ concentration either due to genotype or interaction (genotype $\mathrm{x}$ treatment). That was the case with lines T-083, T-122, T-128, T-135, and T-141. 


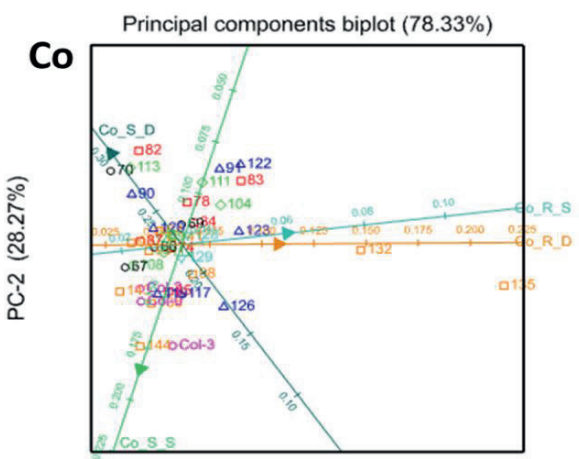

PC-1 (50.06\%)

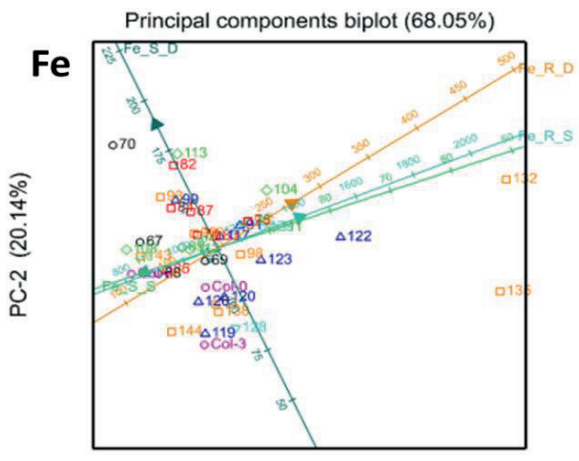

PC-1 (47.91\%)

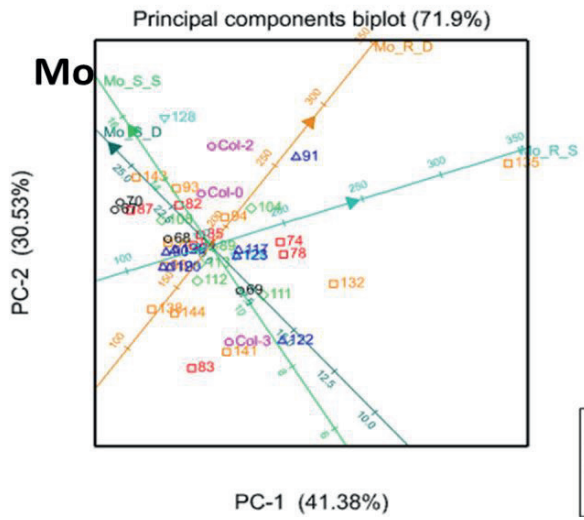

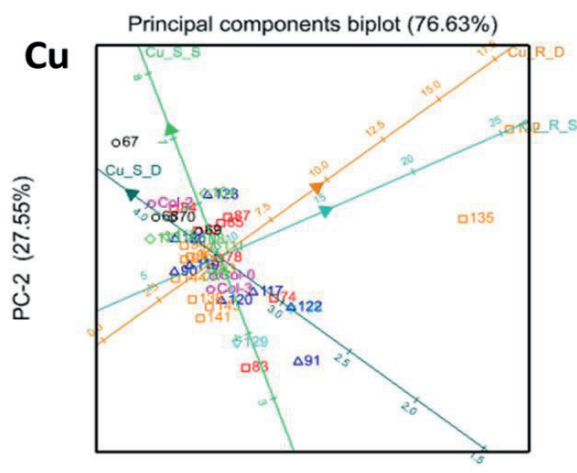

PC-1 $(49.08 \%)$

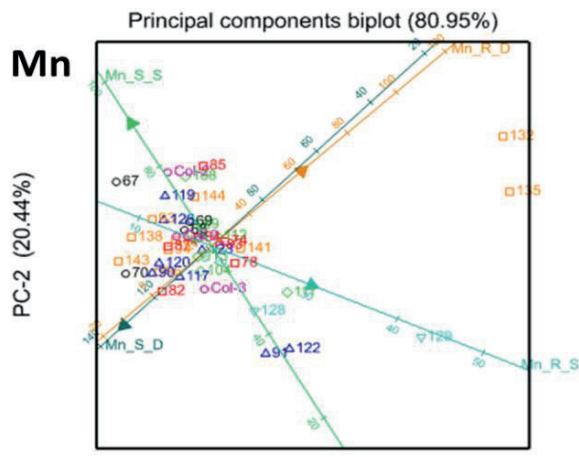

PC-1 $(60.51 \%)$

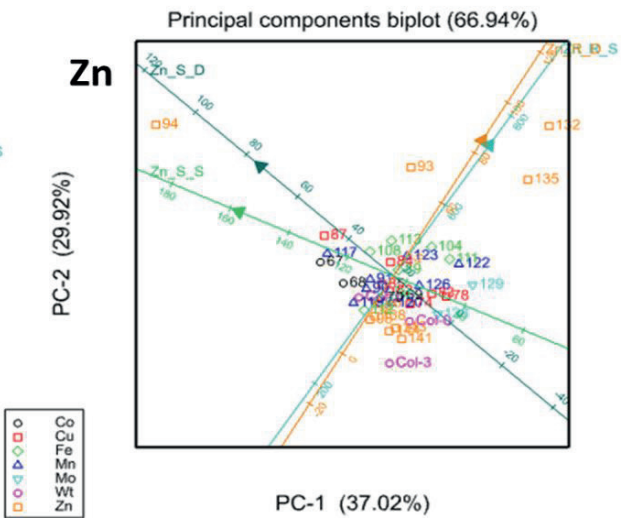

Figure 4. PCA Biplot of element concentrations of T-DNA insertion mutant lines. Concentrations of Co, $\mathrm{Cu}, \mathrm{Fe}, \mathrm{Mn}, \mathrm{Mo}$, or $\mathrm{Zn}$ in shoots (_S_) and roots (_ $R \_$of $T$-DNA insertion mutant lines and wild-type plants grown in Zn sufficiency (S) or Zn deficiency (D). Each mutant is grouped according to the element (symbol with different colour and shape next to the mutant number) to which each candidate gene was associated with in the GWAS. Wild-type (Wt) backgrounds are grouped together and represented with magenta circles. 


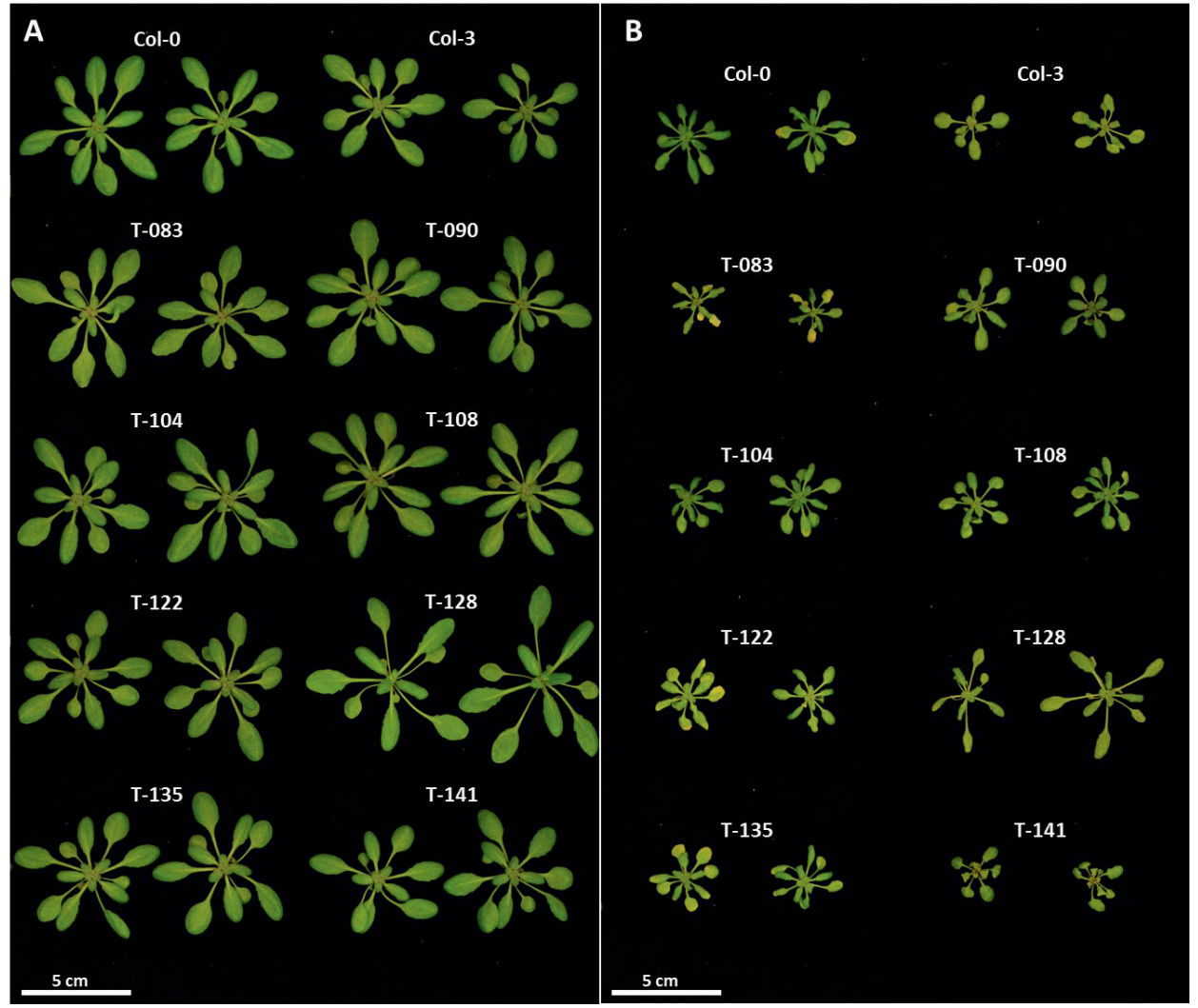

Figure 5. Shoot phenotypes of wild-type Col-0/Col-3 and eight GWAS candidate gene T-DNA insertion mutant lines (T-083, T-090, T-104, T-108, T-122, T-128, T-135 and T-141) grown hydroponically in (A) Zn sufficiency for three weeks, or (B) for one week in Zn sufficiency plus two weeks in Zn deficiency. Mutant T-141 was grown for two additional days in Zn deficiency. 


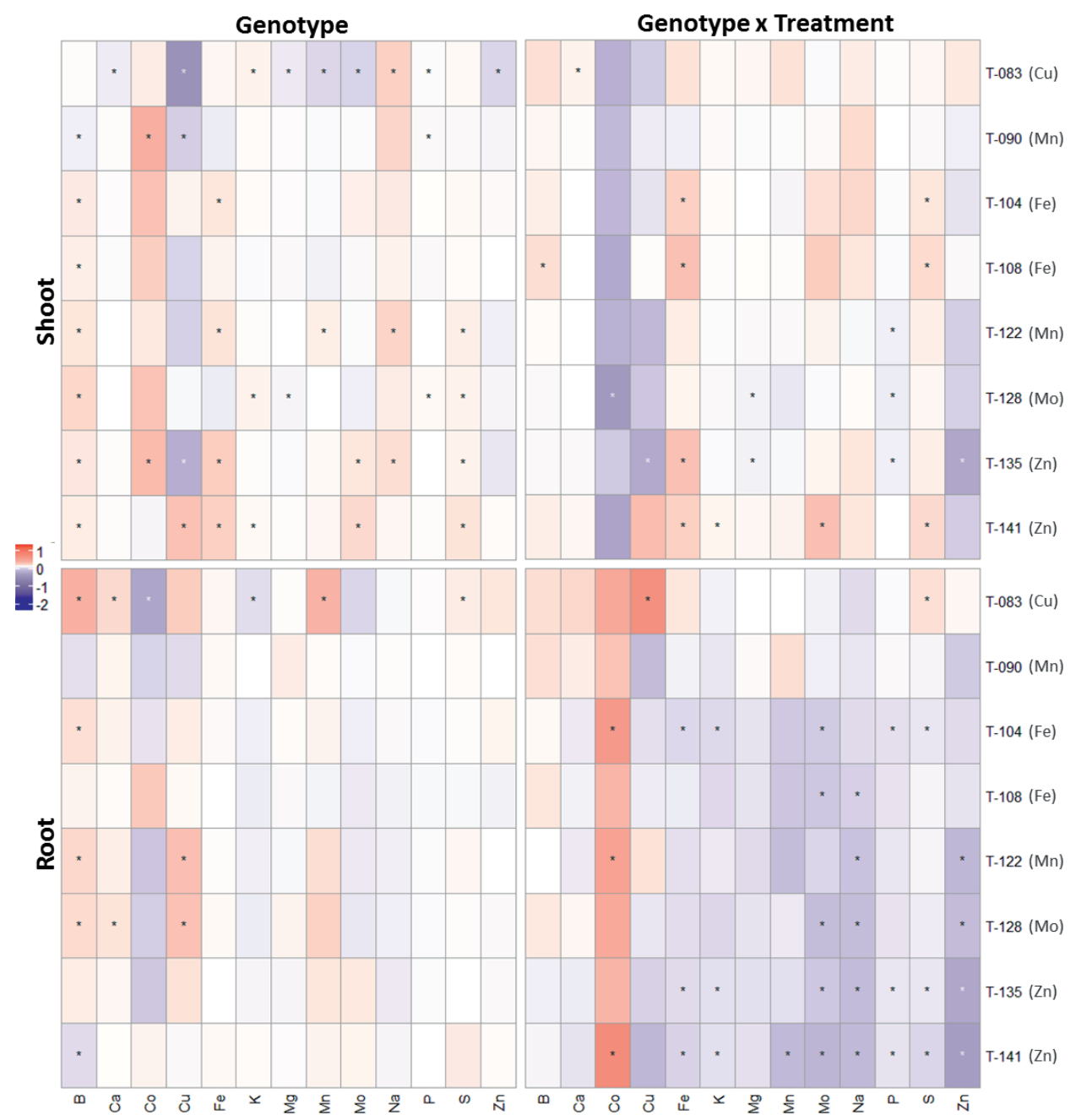

Figure 6. Heatmap of shoot and root element concentration fold-changes of T-DNA insertion mutant lines (T-083, T-090, T-104, T-108, T-122, T-128, T-135, and T-141) compared to wild-type plants. Plants were grown hydroponically in Zn sufficiency or Zn deficiency. First the fold-change due to genotype was calculated $\left(Y_{T D N A, D E F}+Y_{T D N A, S U F}\right) /\left(Y_{W T, D E F}+Y_{W T, S U F}\right)$, second the fold-change due to the genotype and treatment interaction was calculated $\left(Y_{T D N A, D E F} / Y_{W T, D E F}\right) /\left(Y_{T D N A, S U F} / Y_{W T, S U F}\right)$ where $Y$ stands for the mean of a trait value, WT = control genotype, TDNA $=T$-DNA line genotype, DEF = deficiency treatment, SUF=sufficiency. * indicates significant concentration fold-changes with a FDR (False Discovery Rate) adjusted p-value lower than 0.05. Colours indicate positive (red) or negative (blue) fold-changes. The element next to each T-DNA insertion mutant line indicates to which element each candidate gene was associated with in the GWAS. 


\section{Discussion}

\section{Ionome}

The ionom e was defined by David Salt in 2008 as "the m ineral nutrient and trace elem ent composition of an organism, and represents the inorganic component of cellular and organismal system s". The ionome works as a coordinate network where the disequilibrium of one element concentration is reflected in changes in concentrations of several elements of the ionome (Buescher et al., 2010; Baxter et al., 2012; Huang and Salt, 2016) .

The Arabidopsis ionome analyses illustrated that from the thirteen elements quantified, $\mathrm{Zn}$ deficiency increased the concentration in shoots of $\mathrm{B}, \mathrm{Ca}, \mathrm{Co}, \mathrm{Fe}, \mathrm{Mg}, \mathrm{Mn}, \mathrm{Mo}, \mathrm{P}$, and $\mathrm{S}$, and in roots of $\mathrm{B}, \mathrm{Ca}, \mathrm{K}, \mathrm{Na}$, and $\mathrm{S}$. Also the relative change of $\mathrm{Zn}$ concentrations due to $\mathrm{Zn}$ deficiency indicates a decrease 5\% higher in shoots than in roots. On the other hand, the relative change of $\mathrm{Mn}, \mathrm{Mg}$, Mo, and Fe concentrations due to $\mathrm{Zn}$ deficiency shows a decrease in roots and an increase in shoots. The changes in element concentrations and allocations are due to competition among elements with similar chemical characteristics, crosstalk regulation processes, and transporters with overlapping substrate specificities (Huang et al., 2000; Graham, 2008; Nichols et al., 2012; Nishida et al., 2015). Some transporters with overlapping substrate specificities among $\mathrm{Zn}$ and other elements are Iron-Regulated Transporter 1 (IRT1: Fe, Zn, Mn, Cd, Co), Zinc Transporter 1 Precursor (ZIP1: Zn, Mn), ZIP4 (Zn, Cu), Ferric Reductase Defective 3 (FRD3: Fe, Zn), NRAMP3/4 (Fe, Zn, Mn, Cd), Heavy Metal Atpase 1 (HMA1: $\mathrm{Ca}, \mathrm{Cu}, \mathrm{Cd}, \mathrm{Zn}, \mathrm{Co}$ ), and HMA2 (Cd, Ni, Co, Cu, Pb, Zn) (Henriques et al., 2002; Eren and Argüello, 2004; Grotz and Guerinot, 2006; Puig and Peñarrubia, 2009; Sinclair and Krämer, 2012; Milner et al., 2013). In Zn deficiency, several of these transporters such as ZIP1, ZIP4, FRD3, and HMA2 increase their expression (van de Mortel et al., 2006; Campos, 2015). For instance, Fe and $\mathrm{Zn}$ may compete for root to shoot transport by FRD3 and could transport more Fe to shoots as consequence of Zn deficiency (Durrett et al., 2007; Pineau et al., 2012). The shortage of $\mathrm{Zn}$ ions, during $\mathrm{Zn}$ deficiency, can increase the transport of other divalent cations present in the plant instead of $\mathrm{Zn}$. This process could lead to toxicity problems in the plant. Consequently, changes in the concentration of other elements, are also indicators of the level of tolerance to $\mathrm{Zn}$ deficiency in a plant. 


\section{GWAS}

The heritabilities for element concentrations, as determined for the HapMap population of Arabidopsis natural accessions, varied from 0.38 to 0.65 , and was higher for roots than for shoots, and higher in Zn deficiency than in Zn sufficiency. Ionome heritabilities vary widely in Arabidopsis, ranging from 0.18 to 0.96 according to the sampled plant part, the growth conditions and the type of population (Ghandilyan et al., 2009; Atwell et al., 2010; Baxter et al., 2012; Ghandilyan et al., 2012; Campos et al., 2017). The observed heritabilities suggest that this population is amenable for genetic analysis, with a good prospect that genetic loci can be identified, provided there are not too many loci involved, each with a minor effect on the phenotype, in determining the element concentrations. In those cases, polymorphisms do not cause a phenotypic effect large enough to be detected (Manolio et al., 2009; Gibson, 2010; Yang et al., 2010; Korte and Farlow, 2013).

In the relatively few GWAS that have been performed for Arabidopsis ionomes, very few known micronutrient transporters have been detected. In our study, only for two of the analysed elements, a known micronutrient transporter gene was among the candidates. The first was NRAMP1, which was found to be associated with the Mn concentration. It is a known Mn transporter, involved in Mn uptake from the soil (Cailliatte et al., 2010; Castaings et al., 2016), also able to transport Fe and Cd (Curie et al., 2000; Takahashi et al., 2011). The second was MOT1, associated with Mo concentration, and encoding a known molybdate transporter (Tomatsu et al., 2007; Baxter et al., 2008). The same locus has been detected by GWAS before to affect the Mo concentration in Arabidopsis (Atwell et al., 2010). The Mo concentration also had the highest heritability in our study and other genetic studies (Baxter et al., 2012; Campos et al., 2017).

$\mathrm{Zn}$ homeostasis has been investigated in detail in Arabidopsis and several genes encoding $\mathrm{Zn}$ transporters have been identified so far, belonging to the ZIP, MTP/CDF, YSL and HMA families (Grotz et al., 1998; Korshunova et al., 1999; Vert et al., 2001; Hussain et al., 2004; Eide, 2006; van de Mortel et al., 2006; Waters et al., 2006; Sinclair et al., 2007; Lin et al., 2009; Deinlein et al., 2012; Milner et al., 2013). These genes do not give $\mathrm{Zn}$ deficiency related phenotypes, probably due to their genetic redundancy. Although we were expecting to find at least a few of these genes among the candidates associated with $\mathrm{Zn}$ concentration, this was not the case. This confirms the general observation for GWAS of mineral concentrations in plants, that natural genetic variation in mineral transporters rarely contributes to phenotypic variation. 
The main reasons for these results could be the multigenic character of the $\mathrm{Zn}$ deficiency tolerance and redundancy among transporters. This means several loci with small effects each one, including epistatic interactions as the one reported between bZIP19 and bZIP23 (Assunção et al., 2010).

On the other hand, GWAS has been successful to identify genes in Arabidopsis that favour tolerance to high concentrations of non-essential, toxic elements, such as Cd and As. Similar populations as the one we used were grown upon $\mathrm{Cd}$ or As supply. In this way, variation at the $H M A 3$ gene was identified, encoding a P-type ATPase involved in $\mathrm{Zn}$ and $\mathrm{Cd}$ translocation, to be important for $\mathrm{Cd}$ accumulation and tolerance (Chao et al., 2012). It also led to the discovery of the HACl gene, encoding a novel arsenate reductase involved in plant tolerance to As (Chao et al., 2014). The tolerance to non-essential elements requires mainly exclusion of the element from the plant or internal sequestration to detoxify it, instead of maintaining essential elements homeostasis, which is much more about maintaining a steady supply of the element, to keep the internal concentration well between toxicity and deficiency levels. This likely requires functional alleles and often does not favour loss-of-function alleles, as were discovered for the $H M A 3$ and $H A C 1$ genes, which contribute with large effect sizes to the phenotype and are easily detected by GWA (Atwell et al., 2010).

Nevertheless our inability to identify known metal transporters as candidates underlying loci affecting the concentration of the same element, we did identify several known genes encoding proteins involved in homeostasis of another element. Examples of those were KUP11 (Ahn et al., 2004; Bradshaw, 2005), HIPP21 (Tehseen et al., 2010), PAP26 (Robinson et al., 2012), MGT10 (Li et al., 2001) TIP2;3 (Loqué et al., 2005), CASP5 (Tester and Leigh, 2001; Gasber et al., 2011), MOT2 (Gasber et al., 2011), NIP6;1 (Tanaka et al., 2008), MGT1 (Ishijima et al., 2012), bZIP23 (Assunção et al., 2010), FER4 (Arosio et al., 2009; Tarantino et al., 2010), HIPP10/11/12/13/14/23/46 (de Abreu-Neto et al., 2013). Since most of these genes were not further examined with respect to their effect on the particular element concentration with which they were found to be associated, it could still be that they are false positives (Korte and Farlow, 2013) or they could be also affecting the homeostasis of the element they were associated with in the GWAS. 


\section{Validation of candidate genes}

A total of 38 T-DNA insertion mutants disrupting 34 candidate genes were initially tested for any relevant phenotypic effect under Zn deficiency. Almost half of these mutants showed a difference with wild type either in ionome profile or in biomass production (Supplemental figure S4 and S5). The lack of detectable phenotype in the other half could be for several reasons. First and most likely is that the gene is not involved in the trait, because either the associations were false positives or another gene in the LD region of the locus associated is responsible for changes in the ionome. The selection criteria favour the selection of genes with known functions related with nutrients homeostasis. Thus, genes with unknown function could be missed. Second, variants of other genes can play an important role in the effect of a gene due to epistatic interactions, which may not be present in the genetic background of the T-DNA line tested (Weigel, 2012). Third, the evaluated mutants were all generated in the Col-0 or Col-3 background, in which the phenotypic effect may not be very striking, simply because it does not carry a strong wild-type allele of the gene, or it may even carry a natural null allele, in which case the T-DNA insertion allele will not alter the gene function and will not lead to a different phenotype (Koornneef et al., 2004). Therefore, the phenotypic effect of a gene can only be detected in a specific genetic background, which may not always be Col, and this is the common background of T-DNA insertion lines are available.

For a second validation experiment, we selected eight T-DNA insertion mutants, seven based on their differential ionome profile and one based on the observed $\mathrm{Zn}$ deficiency-related changes in biomass, as observed in the first experiment. The TRM22 gene was selected based on associations with $\mathrm{Cu}$ concentrations, the trm 22 mutant showed the strongest $\mathrm{Zn}$ deficiency symptoms of all mutants and wild-type plants and a significant increase of root $\mathrm{Cu}$ concentration due to $\mathrm{Zn}$ deficiency treatment when compared to wild-type plants (Figure 4, 5 and Supplemental Figure S6). The expression of TRM22 is induced in roots and repressed in shoots due to $\mathrm{Zn}$ deficiency (Campos, 2015). The protein encoded by this gene belongs to the TONNEAU1 Recruiting Motif(TRM) protein family (Drevensek et al., 2012). Members of this family are involved in microtubules array organization during cell division (Azimzadeh et al., 2008; Spinner et al., 2010; Mach, 2012). Initially, we tested two trm 22 mutants (T-082 and T083). However, T-082 did not show the same ionome profile as T-083 in the initial screen. The reason could be that T-082 disrupts not just TRM22 but also AT1G24822, a non-described gene. 
The T-104 and T-108 mutants, for At5g66890 and TOE3 respectively, which were selected based on associations with Fe concentrations, had a higher shoot Fe concentration than wild type upon Zn deficiency. The gene At5g66890 encodes a protein which belongs to LRR (Leucine-Rich Repeat) protein family and is a probable disease resistance protein (Meyers et al., 2003). The gene TOE3 encodes a transcription factor of the APETALA 2/Ethylene Responsive Factor (AP2/ERF) superfamily and appears to be a conserved paralogue of the $A P 2$ gene (Zumajo-Cardona and Pabón-Mora, 2016). Both AP2 and TOE3 are involved in controlling flowering time and meristem identity (Jung et al., 2014). It is not directly obvious why mutation of either the LRR-family gene or TOE3 would affect Fe homeostasis. Flowering time and plant defence mechanisms can indirectly affect the Fe homeostasis considering that plant defence mechanisms against pathogens and Fe deficiency response share common features (Zamioudis et al., 2014; Aznar et al., 2015; Martínez-Medina et al., 2017) and that the hypersensitive iron deficiency bhlh100/bhlh101 double mutant displays a late flowering phenotype (Sivitz et al., 2012).

Candidate genes found to be associated with Mn concentration in shoots, were NRAMP1, PDE318, and GLP9. The NRAMP1 gene was not further validated due to its already known function in Mn transport (Cailliatte et al., 2010; Castaings et al., 2016). The PDE318 protein is part of the P-loop containing NTP-ase superfamily. Members of this family hydrolyze nucleoside triphosphates and are involved in diverse cellular functions such as root growth $(\mathrm{Yu}$ et al., 2016), assembly of F/S proteins (Hausmann et al., 2005), negative regulator of plant defense responses (Cheung et al., 2016). In addition, PDE318 has sequence similarities with Fe transport protein $\mathrm{B}(\mathrm{FeoB})$ involved in the Fe transmembrane (tilacoid) transporter (Kammler et al., 1993; Kwon and Cho, 2008). The T-122 pde318 mutant had higher Fe and Mn concentrations in shoots than the wild type, while in roots, $\mathrm{Zn}$ concentration was significantly lower than the wild type upon $\mathrm{Zn}$ deficiency. The GLP9 encodes a germin-like protein. The gene is mainly expressed in roots and can be induced by abiotic stress conditions (Winter et al., 2007) and the defence responses to nematodes (Jammes et al., 2005; Vellosillo et al., 2007). The T-090 glp 9 mutant produced more biomass than the wild type, but we did not observe any effect on the Mn concentration when compared to wild type, however, glp 9 produced more biomass than the wild type.

The MOT1 gene was found to be associated with the Mo concentrations. It encodes a Mo transporter (Tomatsu et al., 2007; Baxter et al., 2008). The ELF3 gene also was associated with 
Mo concentrations. It encodes a component of the circadian clock (Covington et al., 2001). The T-128 elf 3 mutant plants were clearly different from the rest of the genotypes analysed due to its characteristic elongated petioles (Reed et al., 2000) and its insensitivity to photoperiod signals (Song, 2012). There is not a clear link between this gene and Mo concentrations, however, the circadian clock regulates the transcription of $\mathrm{Mg}, \mathrm{Fe}, \mathrm{Mn}$, and $\mathrm{Cu}$ transporters (Haydon et al., 2011). Moreover, element concentrations show circadian oscillations (Johnson et al., 1995; Haydon et al., 2015) and concentrations of $\mathrm{Cu}, \mathrm{Fe}$, and $\mathrm{Mg}$ affect the period length of circadian rhythm (Andrés-Colás et al., 2010; Chen et al., 2013; Hong et al., 2013; Feeney et al., 2016).

Mutants T-135 and T-141, respectively mutated in At1g49350 and FER4, associated with Zn concentrations, showed decreased shoot and root Fe, $\mathrm{P}$, and $\mathrm{Zn}$ concentrations when compared to wild type, due to the effect of the $\mathrm{Zn}$ deficiency treatment. At $\lg 49350$ encodes a pfkB-like carbohydrate kinase with an unknown function. Members of the PkfB family of carbohydrate kinases have diverse carbohydrate substrates as fructose, ribose, adenosine, myo-inositol, and others. The few members of this family characterized are involved in the accumulation of photosynthetic transcripts in chloroplasts, ribose accumulation in both the roots and leaves, and plant growth (Ogawa et al., 2009; Gilkerson et al., 2012; Riggs and Callis, 2016). For instance, the Fe deficiency response requires the accumulation of sugars to induce the expression of $\mathrm{Fe}$ acquisition-related genes (Lin et al., 2016). FER4 gene was associated with Zn concentrations, and encodes a ferritin protein, which acts as Fe storage protein involved in buffering Fe by sequestering intracellular Fe (Arosio et al., 2009; Murgia and Vigani, 2015; Reyt et al., 2015). The Fe concentration was lower in roots and higher in shoots of fer4 compared to wild type due to the $\mathrm{Zn}$ deficiency treatment, alterations in the Fe concentration is characteristic of ferr4 (Tarantino et al., 2010). In wild type, upon $\mathrm{Zn}$ deficiency, the $\mathrm{Fe}$ concentration decreases in shoots and increased in roots. Therefore, the effect of the ferr 4 is an augmented effect of $\mathrm{Zn}$ deficiency. Its loss-of-function deregulates the expression of several element transporters in the plant stem, for instance, several $\mathrm{Zn}$ transporters in the plant stem (Ravet et al., 2009). Apart from it, there is significant decrease in the concentration of $\mathrm{K}, \mathrm{Mn}$, Mo, Na, P, S and increase in Co.

The analysis of the trm22, at5g66890, toe3, elf3, at $1 g 49350$ and ferr4 mutants confirmed a difference with wild type, in the concentration of the same element with which this gene was associated with in the GWAS, in response to Zn deficiency. In addition to the target element, 
the concentration of several elements was altered in these mutants when compared to wild type, due to the $\mathrm{Zn}$ deficiency treatment. Although genetic complementation has not yet been performed, these results suggest that variation at these loci is likely to contribute to variation in the respective mineral concentrations they are implicated in, in response to $\mathrm{Zn}$ deficiency.

\section{Conclusions}

Discovering the molecular basis of complex traits, like micronutrient homeostasis, is a challenge due to the large number of genes involved in the process and the small effect they have on the phenotype, in addition to their redundant functions, epistatic interactions and pleiotropic effects. Zn deficiency does not only affect the plant $\mathrm{Zn}$ concentration, or even $\mathrm{Zn}$ homeostasis, there appear to be several physiological consequences for the homeostatic mechanisms balancing other mineral concentrations in Arabidopsis. Thus, maintaining the balance in the ionome of $\mathrm{Zn}$ deficient plants is also a key point in $\mathrm{Zn}$ deficiency tolerance. As shown in this thesis, the loss of function of seven genes causes an increase in the misbalance of the ionome due to $\mathrm{Zn}$ deficiency. A deep study of these genes is needed to analyse their potential in $\mathrm{Zn}$ deficiency tolerance by maintaining the balance of the ionome.

\section{Acknowledgements}

We thank Dr. Tânia Serra for the help in setting the large scale phenotyping of T-DNA insertion lines. This work was supported by the Ecuadorian government through SENESCYT (Secretaría de Educación Superior, Ciencia, Tecnología e Innovación) and Universidad de las Fuerzas Armadas-ESPE. 


\section{Supplemental information}

Supplemental files link: https://drive.google.com/open?id=12ixp5aiAX8DxrpuW3d-93N-KetWvmze_

Table S1. Description of T-DNA insertion mutants used to validate GWAS candidate genes. The description includes Line ID used as a code in our the experiments, wild-type background, NASC identification (ID) and donor numbers, and locus identifier.

\begin{tabular}{|c|c|c|c|c|}
\hline Line ID & NASC ID & Background & Donor Number & Locus identifier \\
\hline T-067 & N656047 & Col-0 (N60000) & SALK_146824C & At4g26850 \\
\hline T-068 & N671601 & Col-0 (N60000) & SALK_079922C & At4g26970 \\
\hline T-069 & N671156 & Col-0 (N60000) & SALK_009537C & At4g 26970 \\
\hline T-070 & N403166 & Col-0 (N60000) & GK-033H10 & At4g26990 \\
\hline T-073 & N669529 & Col-0 (N60000) & SALK_029940C & At5g17420 \\
\hline T-074 & N655731 & Col-0 (N60000) & SALK_092415C & At5g 17450 \\
\hline T-078 & N413741 & Col-0 (N60000) & GK-144B01 & At5g 34850 \\
\hline T-082 & N663352 & Col-0 (N60000) & SALK_093331C & Atlg67040 \\
\hline T-083 & N673019 & Col-0 (N60000) & SALK_098466C & At1g67040 \\
\hline T-084 & N655835 & Col-0 (N60000) & SALK_111141C & At2g34830 \\
\hline T-085 & N648777 & Col-0 (N60000) & SALK_148777 (BX) & At2g 34830 \\
\hline T-087 & N866736 & Col-0 (N60000) & SALK_010974 & At5g47450 \\
\hline T-089 & N863910 & $\mathrm{Col}-2$ & WiscDsLox262B01 & Atlg24120 \\
\hline T-090 & N872707 & Col-3 & SAIL_270_D05 & At4g14630 \\
\hline T-091 & N662797 & Col-0 (N60000) & SALK_066841C & At4g34950 \\
\hline T-093 & N684026 & Col-0 (N60000) & SALK_049083C & At5g52720 \\
\hline T-094 & N438279 & Col-0 (N60000) & GK-399F11 & At5g52730 \\
\hline T-096 & N667786 & Col-0 (N60000) & SALK_012334C & At5g52740 \\
\hline T-098 & N667818 & Col-0 (N60000) & SALK_013684C & At5g52760 \\
\hline T-104 & N845057 & Col-0 (N60000) & SAIL_1231_B01 & At5g66890 \\
\hline T-108 & N863162 & Col-0 (N60000) & SAIL_603_B01 & At5g67180 \\
\hline T-111 & N673799 & Col-0 (N60000) & SALK_010456C & At1g69550 \\
\hline T-112 & N877090 & Col-0 (N60000) & SAIL_818_D10 & At1g69600 \\
\hline T-113 & N542116 & Col-0 (N60000) & SALK_042116 (AI) & At5g 15290 \\
\hline T-117 & N685506 & Col-0 (N60000) & SALK_025900C & At1g11670 \\
\hline T-119 & N859621 & Col-0 (N60000) & SALK_103884(BD)(BO) & Atlg12950 \\
\hline T-120 & N677058 & Col-0 (N60000) & SALK_136631C & Atlg12950 \\
\hline T-122 & N645367 & Col-0 (N60000) & SALK_145367 (BL)(BX) & At1g80770 \\
\hline T-123 & N669178 & Col-0 (N60000) & SALK_142773C & At1g80780 \\
\hline T-126 & N817417 & CS8846 & SAIL_378_B11 & At4g39700 \\
\hline T-128 & N471954 & Col-0 (N60000) & GK-750E02 & At2g25930 \\
\hline T-129 & N862551 & Col-0 (N60000) & SAIL_335_F05 & At5g59250 \\
\hline T-132 & N662685 & Col-0 (N60000) & SALK_061961C & At5g48250 \\
\hline T-135 & N859815 & Col-0 (N60000) & Salk_006361 & At1g49350 \\
\hline T-138 & N853035 & Col-2 & WiscDsLox366A7_052 & At1g49660 \\
\hline T-141 & N662835 & Col-0 (N60000) & SALK_068629C & At2g40300 \\
\hline T-143 & N661589 & Col-0 (N60000) & SALK_015121C & At2g40435 \\
\hline T-144 & N660899 & Col-0 (N60000) & SALK_058919C & At2g40460 \\
\hline
\end{tabular}


A

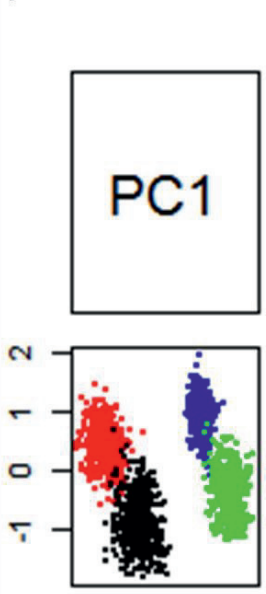

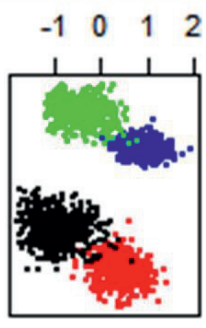

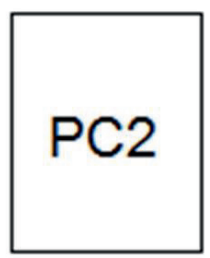

B

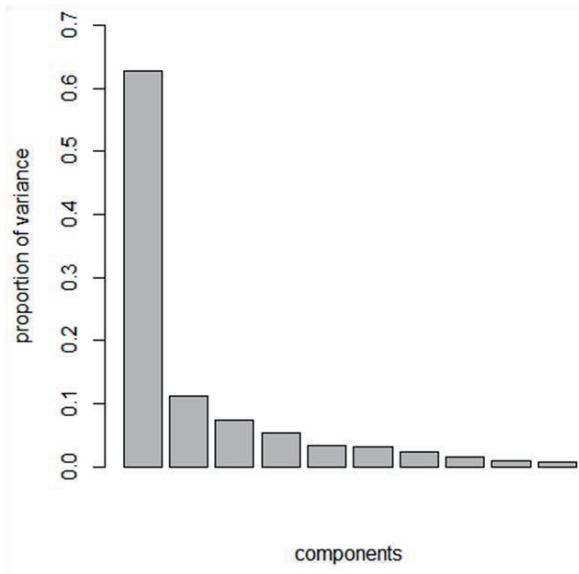

Figure S1. Principal component analyses of the concentrations of 13 essential elements in 350 natural accessions of Arabidopsis. (A) Distribution of accessions based on element concentrations in shoots (Shoot Suf, blue dots) and roots (Root Suf, red dots) of plants grown hydroponically in Zn sufficiency and shoots (Shoot Def, green dots) and roots (Root Def, black dots) of plants grown hydroponically in $Z n$ deficiency, for different combinations of principal components (PCS). (B) Proportion of variance explained by each one of the principal components. 

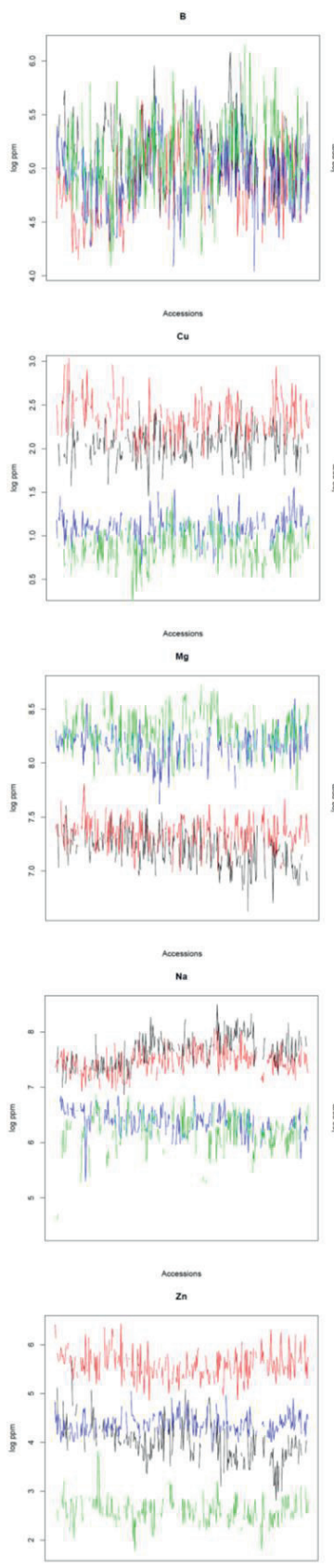
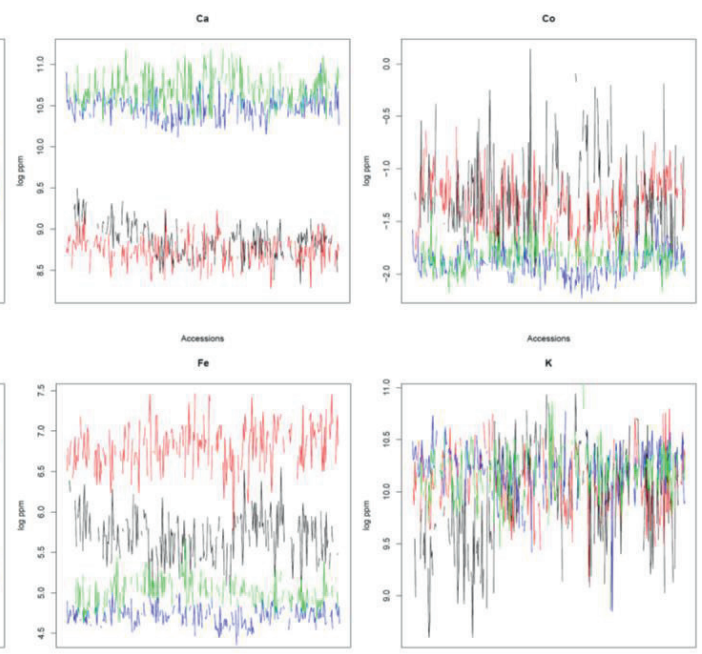

mon
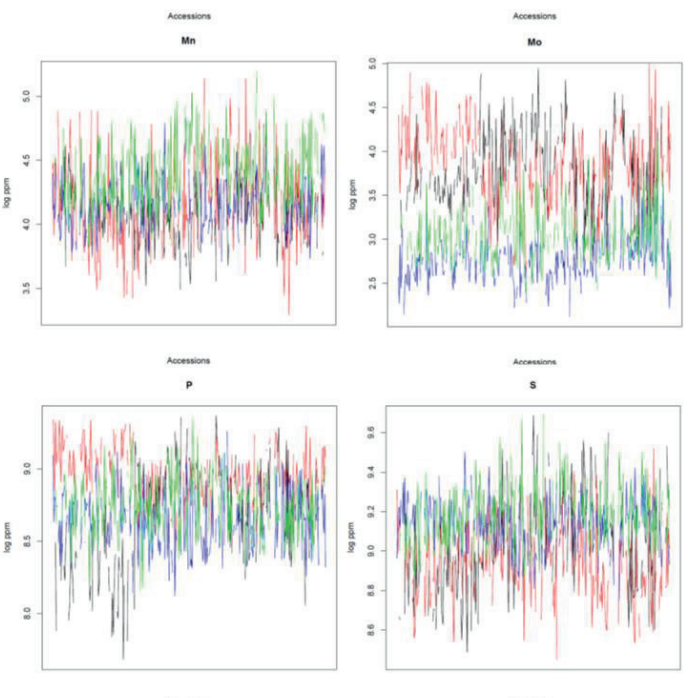

Figure S2. Concentrations of 13 essential elements across 350 natural accessions of Arabidopsis. Element concentrations in shoots (Shoot Suf, blue) and roots (Root Suf, red) of plants grown hydroponically in Zn sufficiency and shoots (Shoot Def, green) and roots (Root Def, black) of plants grown hydroponically in Zn deficiency. 


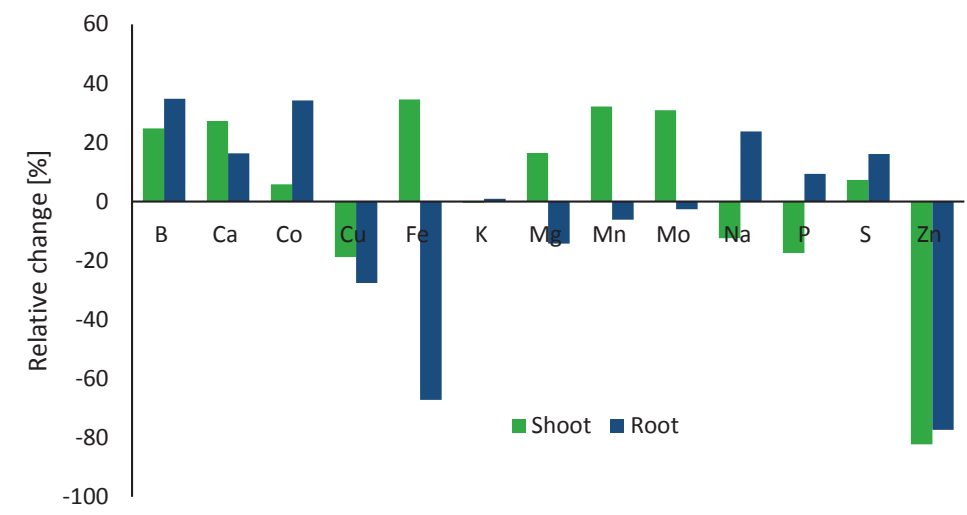

Figure S3. Relative change of the concentrations means of 13 essential elements across 350 natural accessions of Arabidopsis. Relative change in shoots (green) and roots (blue) of plants grown hydroponically in Zn deficiency vs plants grown hydroponically in Zn sufficiency. 

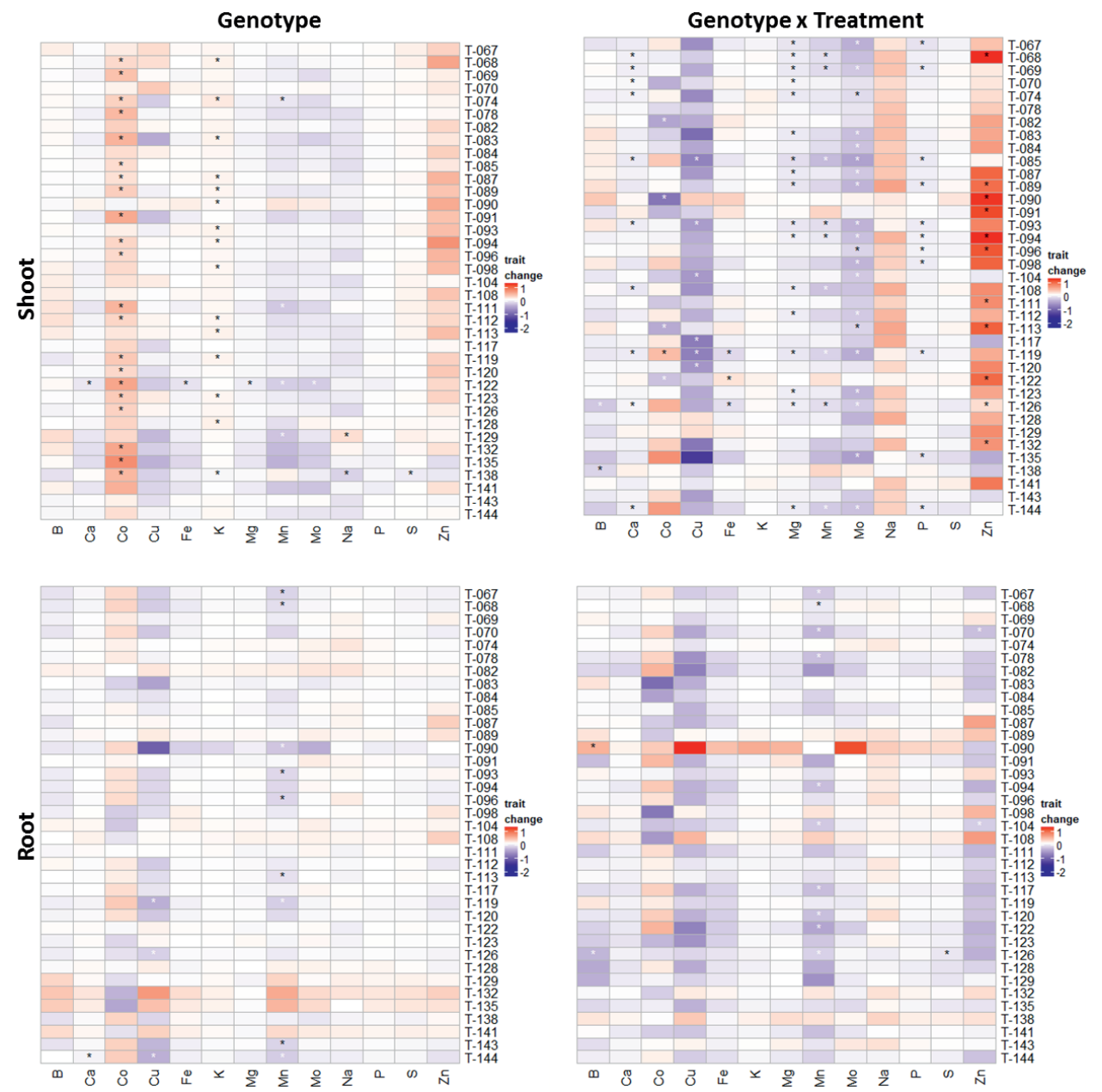

Figure S4. Heatmap representing the ionome profile changes of T-DNA insertion mutant lines. Ionome profile changes of T-DNA insertion mutant lines compared to wild-type plants grown hydroponically in Zn sufficiency or Zn deficiency, from test with the initial 38 T-DNA lines of candidate genes. Significant changes between mutant and wild-type plants denoted with (*) if they had a FDR (False Discovery Rate)-adjusted p-value lower than 0.05. Colours indicate positive (red) or negative (blue) fold changes. First the fold-change due to genotype was calculated $\left(Y_{T D N A, D E F}+Y_{T D N A, S U F}\right) /\left(Y_{W T, D E F}+Y_{W T, S U F}\right)$, second the fold-change due to the genotype and treatment interaction was calculated $\left(Y_{T D N A, D E F} / Y_{W T, D E F}\right) /$ $\left(Y_{T D N A, S U F} / Y_{W T, S U F}\right)$ where $Y$ stands for the mean of a trait value, WT = control genotype, TDNA $=T$ $D N A$ line genotype, $D E F=$ deficiency treatment, $S U F=$ sufficiency. 


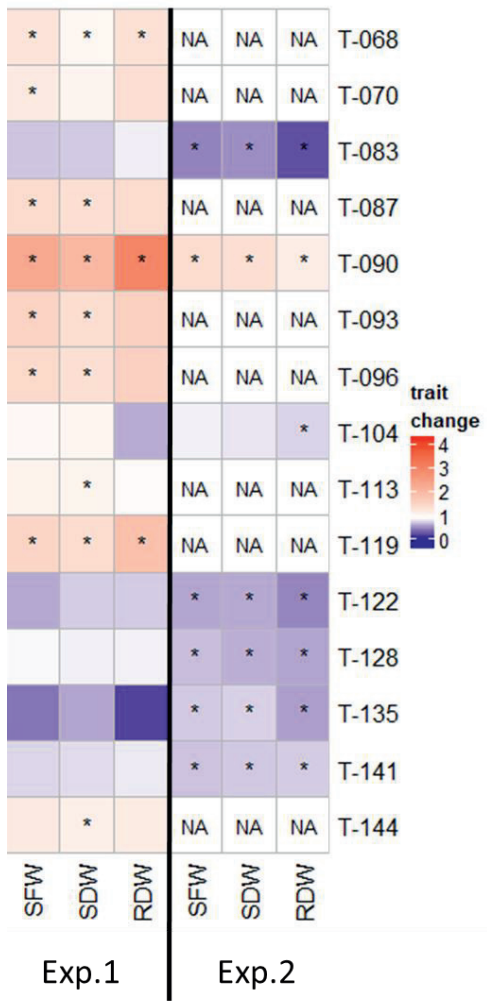

Figure S5. Heatmap of the biomass of T-DNA insertion mutant lines compared to wild-type plants. Plants grown hydroponically in Zn sufficiency or Zn deficiency, as investigated in experiment 1 (Exp.1: initial 38 T-DNA lines) and experiment 2 (Exp.2: selected 8 T-DNA lines). The traits represented are shoot fresh weight (SFW), shoot dry weight $(S D W)$ and root dry weight (RDW). Only mutants with significant differences in biomass when compared to wild type and the effect of the treatment in both experiments. Significance denoted with (*) if they had a FDR (False Discovery Rate)-adjusted p-value lower than 0.05. Colours indicate positive (red) or negative (blue) fold changes when compared the mutant to the wild type. The fold-change due to the genotype and treatment interaction was calculated $\left(Y_{T D N A, D E F} / Y_{W T, D E F}\right) /\left(Y_{T D N A, S U F} / Y_{W T, S U F}\right)$ where $Y$ stands for the mean of a trait value, $W T=$ control genotype, $T D N A=T$-DNA line genotype, $D E F=$ deficiency treatment, $S U F=$ sufficiency. 

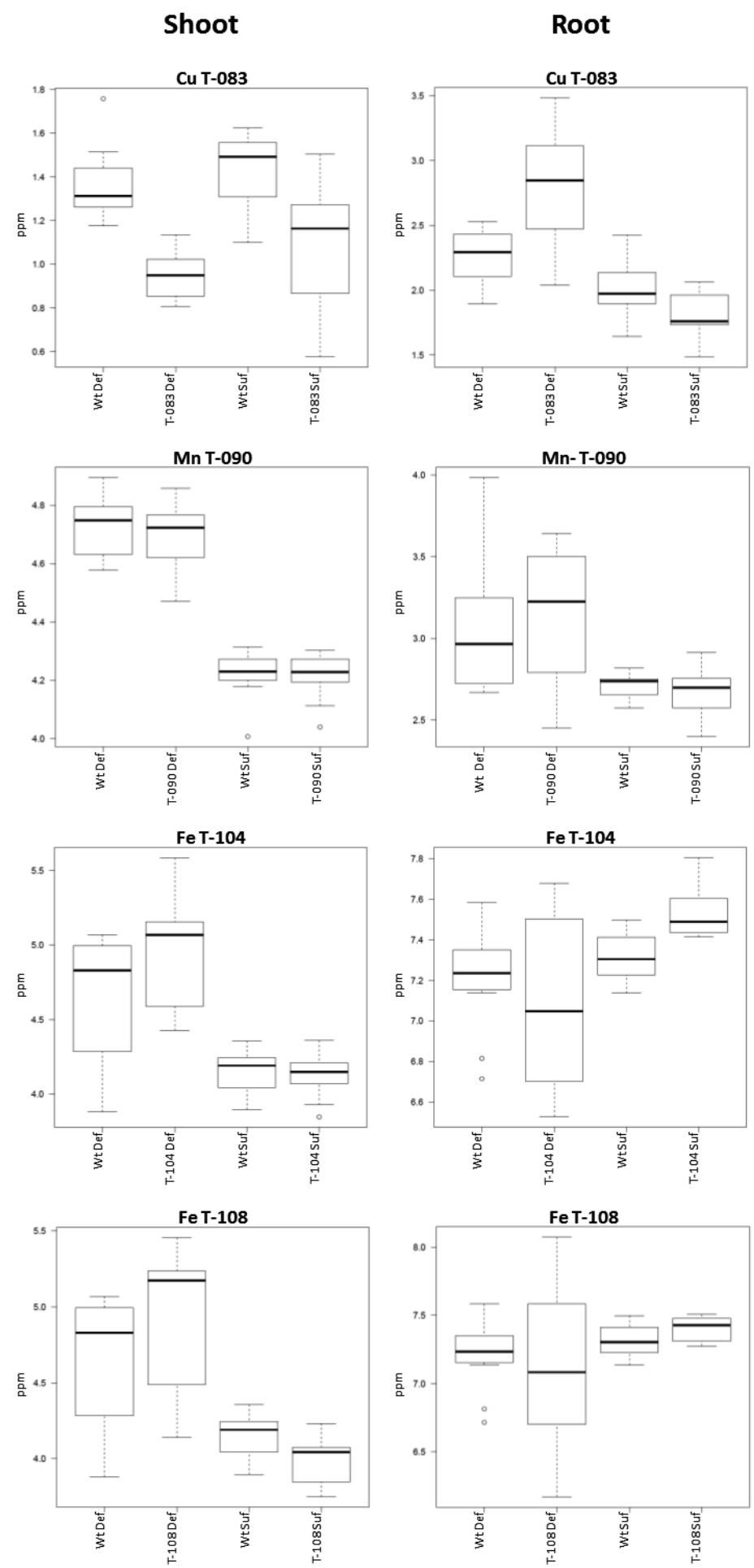
Shoot
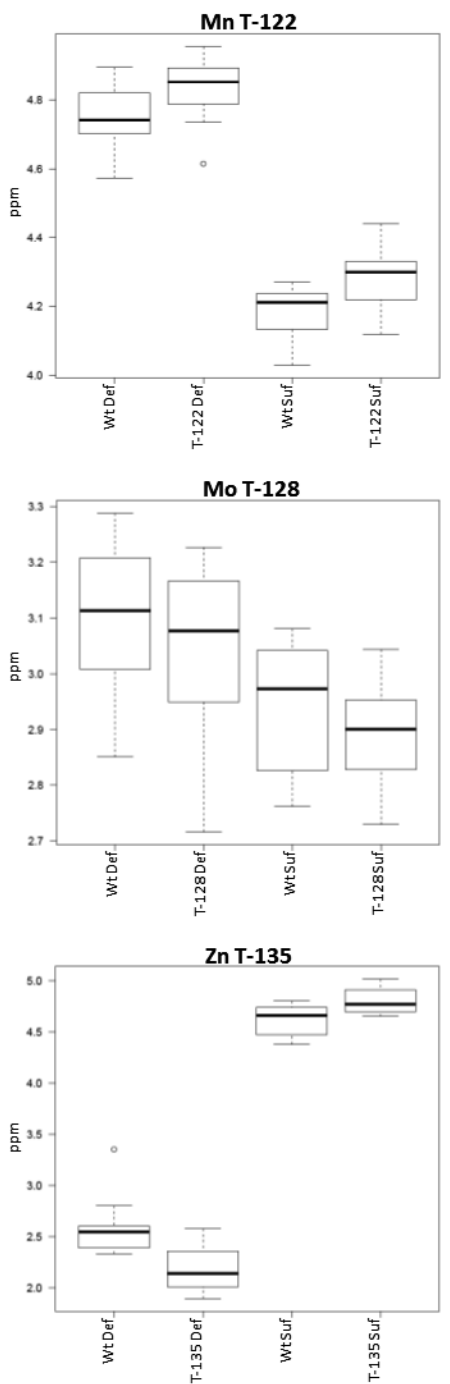

Zn T-141

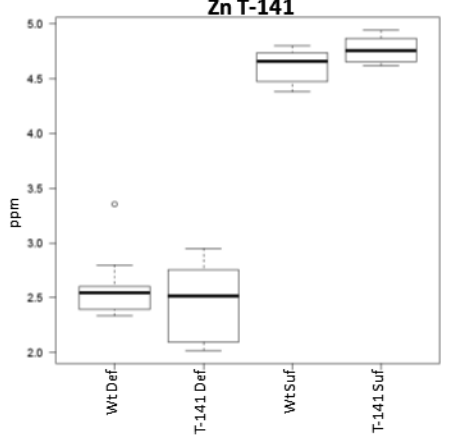

Root
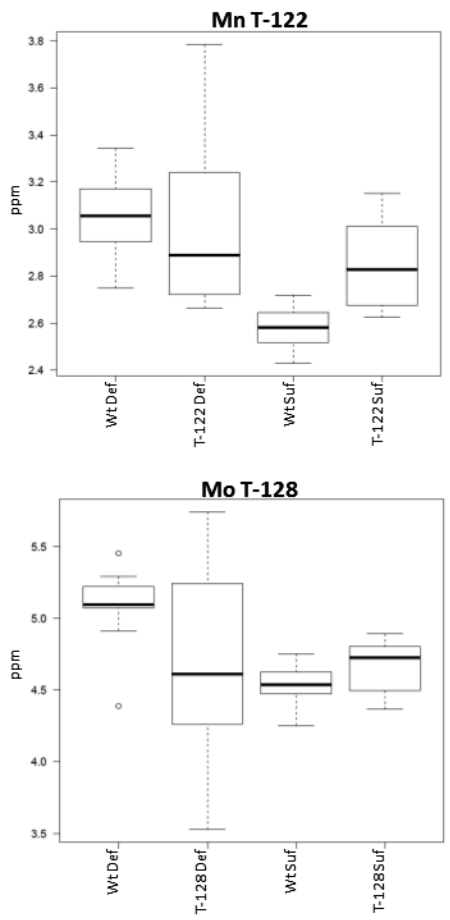

Zn T-135

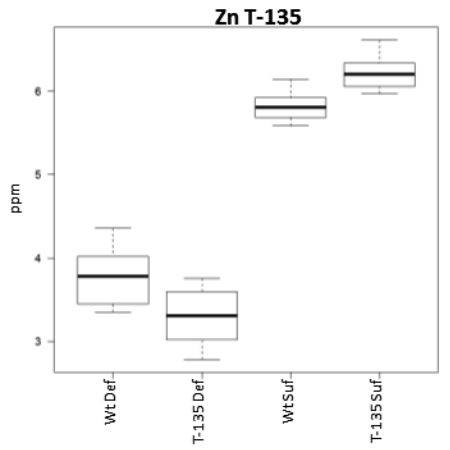

Zn T-141

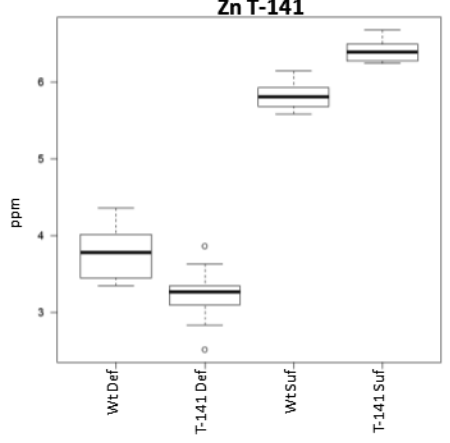


Figure S6. Boxplots of element concentrations of T-DNA insertion mutant lines compared to wild-type plants. Per mutant is plotted the concentrations of the element the mutated gene was associated with in the GWAS. Data represent the $\log ^{e}$ of element concentration of 12 replicate plants per genotype. 




\section{Chapter 5}

\section{A conserver cluster of tandemly arrayed HIPP genes affects tolerance to $\mathrm{Fe}$ and $\mathrm{Zn}$ deficiency in Arabidopsis thaliana}

Valeria Ochoa Tufiño ${ }^{1,2}$, Khadija Aaliya ${ }^{1,3}$, Jos Meeussen ${ }^{1}$ and Mark G.M. Aarts ${ }^{1}$

1) Laboratory of Genetics, Wageningen University, Wageningen, The Netherlands

2) Current affiliation: Departamento de Ciencias de la Vida, Universidad de las Fuerzas Armadas ESPE, Sangolquí, Ecuador

3) Current affiliation: Institute of Agricultural Sciences, University of the Punjab, Lahore, Pakistan

In preparation for submission 


\begin{abstract}
Abiotic stresses decrease the growth and productivity of plants. Stress-tolerant crops are required for agriculture, and the identification of stress-related genes which increase plants tolerance to abiotic stress is important to achieve such. Previously, by a Genome-Wide Association Study (GWAS) we identified a cluster of tandemly arrayed Heavy MetalAssociated Isoprenylated Plant Proteins (HIPPs) to be associated with Zn deficiency tolerance in Arabidopsis thaliana (Arabidopsis). In this study, we explored the relevance of the HIPPI0, HIPP11, HIPP12, HIPP13 and HIPP14 genes of this cluster for the Fe and $\mathrm{Zn}$ deficiency response. The expression of these five HIPPs is induced in plants upon Fe or $\mathrm{Zn}$ deficiency and single mutant lines hipp10, hipp11, hipp12 and hipp14 are less sensitive to Fe and Zn deficiency treatments compared to wild-type plants. In these hipp mutants, the expression of the other HIPP genes in the cluster was not induced by Fe deficiency. The five HIPPs interact with several transcription factors involved in stress response and plant development. Altogether, these HIPPs appear to be negative regulators of plant growth, especially under Fe and $\mathrm{Zn}$ deficiency stress.
\end{abstract}




\section{Introduction}

The bottom of chromosome 5 of Arabidopsis thaliana (Arabidopsis) (coordinates 21363486 to 21390189 bp) contains a cluster of 11 tandemly arrayed genes (from At5g52670 to At5g52770) of which six encode for HEAVY METAL-ASSOCIATED PLANT PROTEINS (HPPs; resp. HPP6, 8, 9, 10, 11 and 12) and five for HEAVY METAL-ASSOCIATED ISOPRENYLATED PLANT PROTEINS (HIPPs; resp. HIPP10, 11, 12, 13 and 14). The region comprising HIPP1014 was previously identified in a Genome-Wide Association Study (GWAS) for response to Zn deficiency to be associated with the root Zn concentration (Chapter 4). HIPPs and HPPs are predicted to be metallochaperone-like proteins, based on sequence similarities. Arabidopsis encodes for 67 metallochaperone-like proteins, 45 HIPPs, and 22 HPPs, containing a predicted Heavy Metal-Associated (HMA) domain. These 67 proteins are classified into two main branches, the first branch contains 46 proteins and the second one 21 proteins (Tehseen et al., 2010). The cluster of 11 tandemly arrayed genes on chromosome 5 encodes proteins that are all found in this second branch, suggesting that these proteins are phylogenetically related and perhaps even derived from one ancestral HIPP/HPP gene. These 11 proteins contain domains with similarity to the HMA domain, even though they lack the CysXXCys motif associated with the metal binding affinities (Tehseen et al., 2010).

All Arabidopsis HIPPs characterized to this moment, have a function related with environmental stress responses. Cold induces the expression of HIPP24/26/23/25 and represses the expression of HIPP27, while HIPP26 expression is also induced by drought and salt exposure (Barth et al., 2009). HIPP26 is also expressed upon Cd stress, its protein can bind Cd, $\mathrm{Cu}$ and $\mathrm{Pb}$, and its overexpression confers Cd tolerance to Arabidopsis (Gao et al., 2009). The triple mutant hipp20/21/22 has increased sensitivity to high concentration of $\mathrm{Cd}$, but accumulates less $\mathrm{Cd}$ than wild-type plants. The $\mathrm{Cd}$-sensitive $y c f 1$ yeast mutant is rescued by expressing either HIPP20, HIPP21, HIPP22 or HIPP27 through binding Cd or facilitating its transport into the vacuoles or out of the cells, either way, making $\mathrm{Cd}$ not available in the cytosol (Tehseen et al., 2010). HIPP6 expression is induced by $\mathrm{Cd}, \mathrm{Hg}, \mathrm{Fe}$, and $\mathrm{Cu}$, and its overexpression confers tolerance to Cd (Suzuki et al., 2002). HIPP3 expression is repressed by drought and ABA, and induced by Pseudomonas infection. Its overexpression affects the regulation of genes involved in biotic and abiotic stress and seed and flower development, and as a consequence HIPP3-overexpressing lines flower later than wild type (Zschiesche et al., 2015). So, in general, HIPPs seem to play a role in regulatory networks important for stress 
response and development, and their capacity to bind transition metal ions could be important in the response to metal stress, though their function is clearly not limited to metal-exposurerelated responses.

Exposure of plants to environmental stress conditions affect their physiology in many different aspects. To ensure their survival, plants need to coordinate growth and stress responses. Most commonly, plants shut down growth under stress (Huot et al., 2014; Haina and Uwe, 2017). Phytohormones such as gibberellic acids (GAs) and brassinosteroids (BRs) are implicated in stress-responsive plant growth. Vegetative growth, in general, is promoted by GA. GA signalling can be repressed by DELLA proteins, which are transcription factors that restrict plant growth (Silverstone et al., 1997; Sun, 2010). The growth-repressing effects of DELLAs are enhanced by stress signals (Achard et al., 2008; Achard and Genschik, 2009) such as the ones induced by salt stress. The growth reduction observed upon salt stress depends on a GADELLA-mediated mechanism, based on reduced GAs accumulation, which causes the increased DELLA accumulation. Arabidopsis encodes five DELLA proteins, which act redundantly, with the DELLA quadruple mutant gai/rga/rgll/rgl2 showing lower sensitivity to salt stress compared to wild-type plants (Achard et al., 2006). The other group of growthpromoting phytohormones comprises the BRs. One of the final steps in the BR signal transduction pathway is the activation of BRI1-EMS-SUPPRESSOR 1 (BES1) (Clouse, 2011). This transcription factor is a master regulator of the BR pathway, promoting plant growth through the regulation of transcription of several downstream genes. BES1 is degraded upon drought and carbon starvation, which slows down plant growth, while the constitutive activation of BR responses increases the susceptibility to drought. The degradation of BES1 under stress conditions is required for the plant survival (Nolan et al., 2017).

Growth repression upon stress does not depend solely on phytohormones that promote growth but also on the crosstalk with phytohormones that mediate stress responses, such as abscisic acid (ABA), salicylic acid (SA), jasmonic acid (JA) and ethylene (Verma et al., 2016). Disruption of the ethylene signalling pathway promotes vegetative growth (Gagne et al., 2004), while ethylene, JA, and ABA can act together to induce the expression of the ETHYLENERESPONSIVE ELEMENT BINDING FACTOR 4 (ERF4) (Yang et al., 2005). The expression of ERF4 induces the expression of genes involved in biosynthesis and degradation of chlorophyll. ERF4 is also known to be involved in inhibiting plant growth under Fe deficiency and in the control of the expression of Fe uptake transporter genes (Liu et al., 2017). 
In this study, we further investigated the role of HIPP10-HIPP14 in the response of Arabidopsis to $\mathrm{Zn}$ deficiency. We found the regulation and activity of these HIPPs to be related to plant growth upon $\mathrm{Zn}$ and Fe deficiency. The loss of function of either HIPP10, HIPP11, HIPP12 or HIPP14, promotes tolerance to unfavourable supply of both $\mathrm{Zn}$ and $\mathrm{Fe}$, especially to $\mathrm{Fe}$ deficiency. CaMV 35S-mediated overexpression of HIPPs led to smaller plants, with delayed flowering and increased sensitivity to unfavourable supply of both $\mathrm{Zn}$ and $\mathrm{Fe}$, when compared to wild-type plants. We used the HIPPs for yeast two-hybrid (Y2H) screens and identified several transcription factors as interacting partners involved in plant growth, plant development, and stress response. Our results thus provide the first functional analyses of this type of clustered HIPPs, which appear to be negative regulators of plant growth, especially under $\mathrm{Fe}$ and $\mathrm{Zn}$ deficiency stress.

\section{Materials and Methods}

\section{Plant material}

The T-DNA insertion mutant lines for HIPP10 (At5g52720: SALK_049083C), HIPP11 (At5g52730: GK-399F11), HIPP12 (At5g52740: SALK_012334C) and HIPP14 (At5g52760: SALK_013684C) were ordered from NASC (www.arabidopsis.info/BasicForm) (Chapter 4 Table S1). Arabidopsis accession Columbia (Col-0) was used for transformation and as a wildtype control.

\section{Growth conditions}

Prior to germination, seeds were stratified for three days at $4^{\circ} \mathrm{C}$ in the dark. The plants were grown on rockwool, in a growth chamber set at 70\% humidity, a 16/8 h light/dark cycle and $20 / 18^{\circ} \mathrm{C}$ day/night temperatures. For agar-plate-based phenotypic screens, plants were grown on vertical agar plates in a climate-controlled growth chamber set at 50\% humidity, a 16/8 h light/dark cycle and $22 / 20^{\circ} \mathrm{C}$ day/night temperatures. Seeds were surface-sterilized using vaporphase seed sterilization (Clough and Bent, 1998) and sown on 12-cm square petri dishes containing 1\% agar-solidified half-strength MS medium ( $\mathrm{pH}$ 5.8). The $\mathrm{Zn}$ and Fe concentrations in the medium varied from $15 \mu \mathrm{M} \mathrm{ZnSO}_{4}$, and $50 \mu \mathrm{M} \mathrm{Fe}(\mathrm{Na})_{2} \mathrm{EDTA}_{2}$ in control medium to no added $\mathrm{Zn}$ for deficiency and $150 \mu \mathrm{M} \mathrm{ZnSO}_{4}$ for $\mathrm{Zn}$ excess; and no added Fe for deficiency and $250 \mu \mathrm{M} \mathrm{Fe}(\mathrm{Na})_{2} \mathrm{EDTA}_{2}$ for Fe excess. Plants were also grown hydroponically, in a climatecontrolled growth chamber set at $70 \%$ humidity, a $12 / 12 \mathrm{~h}$ light/dark cycle and $20 / 15^{\circ} \mathrm{C}$ day/night temperatures. Vapor-phase-sterilized seeds were sown on $0.55 \%$ agar-filled tubes, of 
which the bottom was cut off, and grown on a modified half-strength Hoagland's nutrient solution (Schat et al., 1996). During two weeks, plants were grown in a control medium (2 $\mu \mathrm{M}$ $\mathrm{ZnSO}_{4}$ ), thereafter, plants continued to grow on the control medium, or they were transferred to the same medium to which no $\mathrm{Zn}$ was added, to induce $\mathrm{Zn}$ deficiency.

\section{RNA isolation and qRT-PCR}

Total RNA was extracted from shoots (from petri dishes $\sim 10$ plants per sample, from hydroponics two fully expanded opposite leaves from 3 plants per sample) using the Directzol ${ }^{\text {TM }}$ RNA MiniPrep Kit (Zym o Research;www.zymoresearch). cDNA was synthetized with iScript cDNA Synthesis Kit (Bio-Rad, www.bio-rad). Gene expression was quantified by realtime monitored quantitative reverse transcriptase PCR (qRT-PCR) using a SYBR $®$ Green mix (Bio-Rad, www.bio-rad). Primers for HIPP and housekeeping genes are described in table S1.

\section{Plasmid Constructs and Generation of Transgenic Plants}

Coding sequences of HIPP10, 11, 12, 13 and 14 were PCR-amplified from Arabidopsis Col-0 cDNA with the Phusion Flash High-Fidelity PCR Master Mix (Thermo Scientific; www.thermofisher) using the primers described in table S2. Fragments were cloned into the pDONR201 vector and subsequently into the expression vector pFAST-R2 (Shimada et al., 2010) using standard Gateway technology (Invitrogen, www.thermofisher.). Constructs were Sanger-sequenced to confirm correct cloning (GATC-BIOTECH; www.gatc-biotech). Arabidopsis plants were transformed following the method described in Clough and Bent (1998). Transformed seeds were selected based on their red fluorescence (Shimada et al., 2010). Experiments were performed with transgenic T2 (homo- and heterozygous) seeds.

\section{Yeast two-hybrid}

The bait expression vectors pDEST32, containing the coding sequences of either HIPP10, 11, 12,13 or 14 were constructed from the pDONR201 clones as described in the previous section. Yeast strain PJ49-4 $\alpha$ was transformed with each bait vector and the Y2H screening assay was performed based on the GAL4 TF reporter system, using the methods described by de Folter and Immink (2011). The transformed PJ49-4 $\alpha$ strain was $m$ ated with the PJ49-4A strains of the Arabidopsis TF library (Pruneda-Paz et al., 2014). An autoactivation test was performed on Synthetic Defined -Leucine/-Histidine (SD -LH) medium supplemented with 1, 5, or $10 \mathrm{mM}$ of 3-amino-1,2,4-triazole (3-AT). The library was screened on SD glucose-Leucine/Tryptophan/Histidine (SD glu-LWH) medium supplemented with 1, 5, and $10 \mathrm{mM}$ 3-AT; and 
SD glu-Leucine/-Tryptophan/-Adenine (SD glu-LWA) medium. Positive interactions were scored after 3 to 5 days of growth, with a threshold of at least five colonies per diploid yeast. For all steps, including the library screen, yeast was grown at $30^{\circ} \mathrm{C}$. The network of positive interactions was built with Cytoscape (Shannon et al., 2003).

\section{Domain search and interaction prediction}

HIPP DNA sequences were retrieved from The Arabidopsis Information Resource (TAIR) (Berardini et al., 2015) (www.arabidopsis.org). MotifScan (Pagni et al., 2007) was used to search for protein motifs, using all possible motif sources and only taking strong matches into account. C-terminal prenylation motifs were predicted using Prenylation Prediction Suite (PrePS) (Maurer-Stroh and Eisenhaber, 2005). Disordered binding regions were predicted using ANCHOR (Dosztányi et al., 2009). Illustrator for Biological Sciences (IBS) 1.0.2 (Liu et al., 2015) was used to make the visual representation.

\section{Statistical analysis}

Student's t-test, one way ANOVA, two way ANOVA and post hoc test were calculated with Genstat 18th edition (VSN International; www.vsni.co.uk/software/genstat/).

\section{Results}

Variation in Arabidopsis root $\mathrm{Zn}$ concentration upon $\mathrm{Zn}$ deficiency is associated with a cluster of five tandemly arrayed HIPP genes

To investigate the genetics underlying the $\mathrm{Zn}$ deficiency tolerance natural variation, we performed a GWAS of $\mathrm{Zn}$ concentration in a set of natural accessions of Arabidopsis exposed to $\mathrm{Zn}$ sufficient and $\mathrm{Zn}$ deficient conditions (Chapter 4). One of the loci associated with the $\mathrm{Zn}$ concentration in roots upon $\mathrm{Zn}$ deficiency, is characterized by the presence of a cluster of eleven tandemly arrayed genes encoding for proteins described as metallochaperone-like (Tehseen et al., 2010). Five genes from this cluster annotated as HEAVY METAL-ASSOCIATED ISOPRENYLATED PLANT PROTEIN (HIPP) genes encode a protein with an isoprenylated domain, while the other six genes, annotated as HEAVY METAL-ASSOCIATED PLANT PROTEIN (HPP), lack this domain. The HIPP genes (HIPP10, 11, 12, 13 and 14) are in linkage disequilibrium ( $\mathrm{LD} ; \mathrm{r}^{2}=0.4$ ) with the $\mathrm{Zn}$-concentration-associated SNPs (Figure 1). 


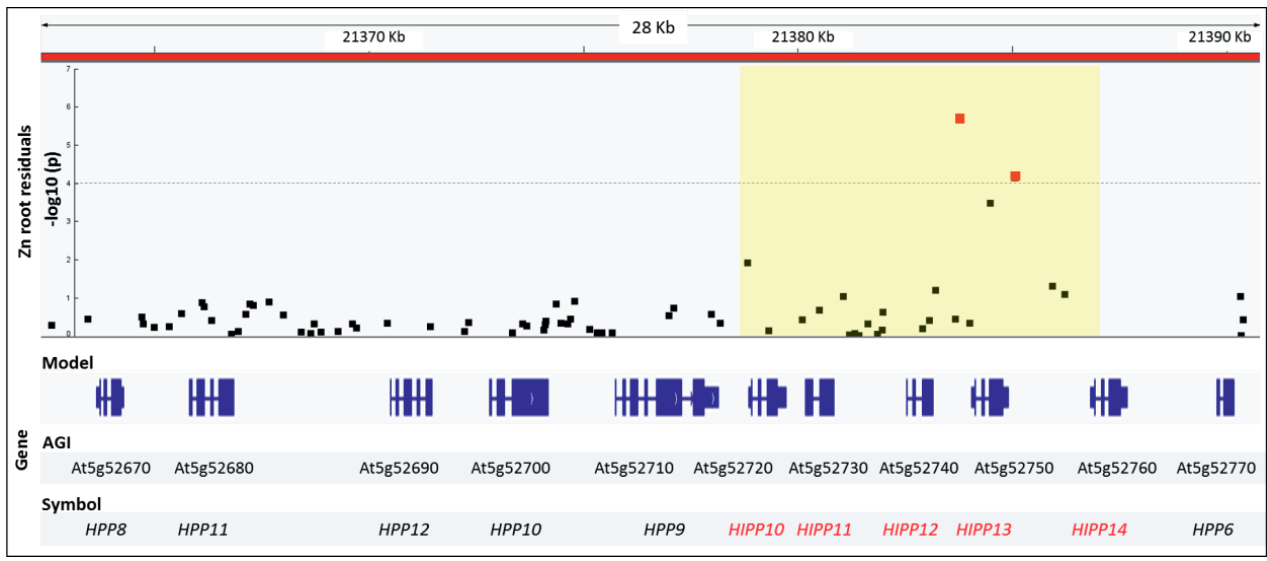

Figure 1. Locus associated with the residuals of the root Zn concentration when comparing plants grown under Zn deficiency, with plants grown under Zn sufficiency, as obtained by GWAS (Chapter 4). The "Manhattan" plot of a 28-Kb region on chromosome 5 (scale shown on top) shows two SNPs (red squares) with association $-\log _{10}(p)$ values above an arbitrary significance threshold set at $-\log _{10}(p)=$ 4 (dashed line), the region in linkage disequilibrium ( $L D \geq 0.4$ )with the two SNPs is inside the yellow area. The gene structures, with gene locus identifier (AGI: Arabidopsis Genome Initiative) and gene symbol (HPP and HIPP) are shown for all genes in this area (tall blue boxes indicating exons, short blue boxes indicating untranslated transcribed regions, and blue lines indicating introns). HIPP genes found in LD with the associated SNPS are indicated in red.

To validate the role of the five HIPP genes in this locus we used hipp10, hipp11, hipp12, and hipp14 mutants (Figure 2A). T-DNA insertion lines hipp10, hipp 11, hipp12 and hipp14 were identified (www.arabidopsis.org) ordered, grown and gene expression was determined (Supplemental Figure S1). The hipp10 and hipp14 lines did not produce any detectable levels of HIPP10 and HIPP14 transcripts, respectively. The hipp 11 line does not include the first exon in the transcript but overexpresses the second exon. All three of these lines are considered to be non-functional, null mutants. The hipp12 line, with a T-DNA insertion in the promoter region, showed similar levels of HIPP12 transcript as the wild type when plants are grown on $\mathrm{Fe}$ sufficient medium, however, the HIPP12 expression is significantly lower in hipp12 mutant compared to wild type on Fe deficient medium. Unfortunately, no suitable T-DNA insertion line for HIPP13 was available. Previous analysis already showed that the hipp10, hipp 11, and hipp 12 mutants had a higher Zn concentration in shoots and higher shoot biomass than wildtype plants upon Zn deficiency (Chapter 4; Figure 4, Supplemental Figures S4, and S5). Moreover, based on transcriptomic data (Chapter 3), transcription of HIPP10, HIPP12, HIPP13 and HIPP14 increases upon exposure to Zn or Fe deficiency (Supplemental Figure S2). Based 
on these results, we decided to further investigate the role of the five HIPP genes not just for their involvement in $\mathrm{Zn}$ homeostasis, but also in Fe homeostasis.

\section{Single HIPPs loss of function promotes growth in stressful $\mathrm{Zn}$ and Fe supply conditions}

To investigate the relationship between the HIPPs and mineral homeostasis, we evaluated the response of hipp10, hipp11, hipp12, hipp14 mutants and 35Sp::HIPP10 plants to Zn and Fe deficiency and excess supply concentrations (Figure 2). When hipp10 and hipp12 mutants were grown on control medium, almost no discernible differences from the wild type were observed, while the hipp 14 mutant was larger than the wild type. Instead, hipp 11 and the 35Sp::HIPP10 plants were smaller than the wild type (Figure 2B and C), although, in a subsequent experiment, there was no longer a significant difference between hippl1 and the other hipp mutants (Supplemental Figure S3). When Zn was left out of the nutrient supply or provided in excess, while keeping Fe supply optimal, wild-type plants showed a decrease in shoot size and slight chlorotic leaves, when compared to plants grown under control conditions. Under $\mathrm{Zn}$ deficiency and Zn excess conditions, hipp10, hipp11, hipp12 and hipp14 mutants showed no signs of stress and grew very similar to the plants on control medium, while the $35 \mathrm{Sp}:$ HIPP 10 plants showed the same Zn deficiency symptoms as wild-type plants (Figure 2B and C and Supplemental table S3). When plants were grown under Fe deficiency, at optimal Zn supply, both wild-type and 35Sp::HIPP10 plants showed an extreme inhibition of root and shoot growth with chlorotic cotyledons compared to plants grown under control conditions. Again, the hipp10, hipp11, hipp12 and hipp14 mutants showed no symptoms of Fe deficiency. Under Fe excess conditions, all genotypes showed inhibition of root and shoot growth and senescing leaves. The hipp10 and hipp14 mutants were the least affected by this treatment. There was a significant effect of genotype, treatment, and genotype $\mathrm{x}$ treatment on plant growth in the different $\mathrm{Zn}$ and Fe deficiency and excess conditions, as calculated by a two-way ANOVA ( $\mathrm{p}$ $<0.001$ for Zn excess, Fe deficiency, and Fe excess; $\mathrm{p}<0.05$ for Zn deficiency). Altogether these results indicate that single mutants of either hipp10, hipp 11, hipp 12 or hipp14 causes an increased tolerance to unfavourable supply of both $\mathrm{Zn}$ and $\mathrm{Fe}$, but are not notably affected in growth under control (optimal) nutrient supply. 
A hipp10
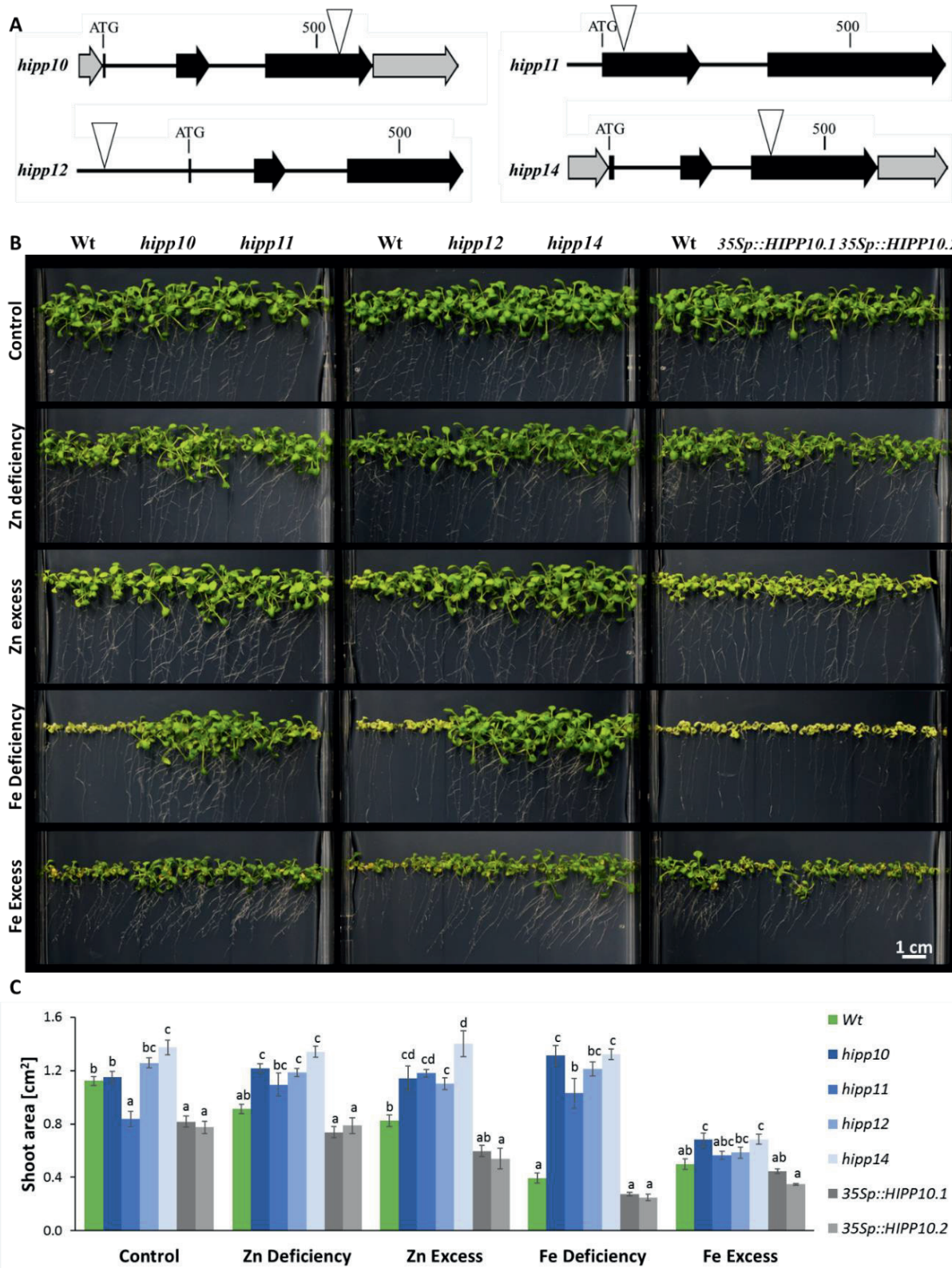

Figure 2. HIPPs mutants are tolerant to unfavourable Zn and Fe supply. (A) Schematic drawings of the HIPP10, HIPP11, HIPP12 and HIPP14 gene sequences illustrating exons (black arrows), UTRs (grey arrows), untranscribed regions (including introns; black line), and the positions of the T-DNA insertions (white triangle). Distance to the start codon (ATG) is indicated above each gene; (B) Wild-type (Wt), hipp mutants and 35Sp::HIPP10 plants grown for three weeks on vertical agar plates with control medium (containing $15 \mu \mathrm{M} \mathrm{ZnSO}{ }_{4}$ and $50 \mu \mathrm{M} \mathrm{Fe}(\mathrm{Na})_{2} \mathrm{EDTA}$ ), or medium imposing $\mathrm{Zn}$ deficiency (no 
added $\mathrm{Zn}$ ), $\mathrm{Zn}$ excess (15 $\mu \mathrm{M} \mathrm{ZnSO}_{4}$ ), Fe deficiency (no added $\mathrm{Fe}$ ) and $\mathrm{Fe}$ excess (250 $\mu \mathrm{M}$ $\mathrm{Fe}\left(\mathrm{Na}_{2} \mathrm{EDTA}_{2}\right.$ ) conditions; (C) Projected shoot area of wild-type (green bars), hipp mutant (blue bars palette), and 35Sp::HIPP10 (grey bars) plants was measured with Image J from plants grown on vertical agar plates (as shown in B). Mean $\pm S E, n=6$ of $\sim 4$ plants each. Letters above the bars denote statistically different groups, when comparing among genotypes grown in the same treatment, obtained with a Bonferroni post hoc test $(\alpha=0.05)$, after a one-way ANOVA $(p<0.001)$.

\section{HIPP gene expression is induced by $\mathrm{Zn}$ and Fe deficiency}

Transcriptome data (Supplemental figure S2) showed that the expression of HIPP12, HIPP13 and HIPP14 was higher in shoots than in roots, while the expression of HIPP10, as well as the expression of the closely linked HPP8, HPP9, HPP10, HPP11 and HPP12 genes (as shown in figure 1), was highest in roots. Expression of HPP6 and HIPPI1 could not be detected in this dataset. The transcription of HIPP12, HIPP13 and HIPP14 was increased in shoots upon exposure to $\mathrm{Fe}$ or $\mathrm{Zn}$ deficiency, the expression of HIPP10 was only induced in shoots by $\mathrm{Zn}$ deficiency. The expression of HPP9 was not affected by $\mathrm{Fe}$ or $\mathrm{Zn}$ variations in the medium, while the expression of HPP8, HPP10, HPP11, HPP12 and HIPP10 was slightly induced in roots by Fe deficiency. This initial analysis suggested that while the expression of HIPP12, 13 and 14 was very similar, it was different from the expression of HIPP10. To confirm the effect of $\mathrm{Zn}$ and Fe deficiency on the expression of the HIPP genes, wild-type plants, grown on agar plates (control and Fe deficient) or hydroponically (control and $\mathrm{Zn}$ deficient), were used for qRT-PCR. This confirmed the higher expression of HIPP10, HIPP11, HIPP12, HIPP13, and HIPP14 in shoots of plants grown on $\mathrm{Zn}$ deficient medium, when compared to $\mathrm{Zn}$ sufficient medium (Figure 3A). Expression of HIPP10 and HIPPII was very low and was only detected upon $\mathrm{Zn}$ deficiency. When plants were grown on Fe deficient medium, only the expression of HIPP10, HIPP12, HIPP13 and HIPP14 was higher than under control conditions (Figure 3B). Differences in expression of HIPP11 were not significant, while the expression of HIPP10 and HIPP12 was mainly detected upon Fe deficiency. There are differences in HIPP expression between the hydroponically grown plants and the agar-plate plants, the latter were generally younger than the former. 
A

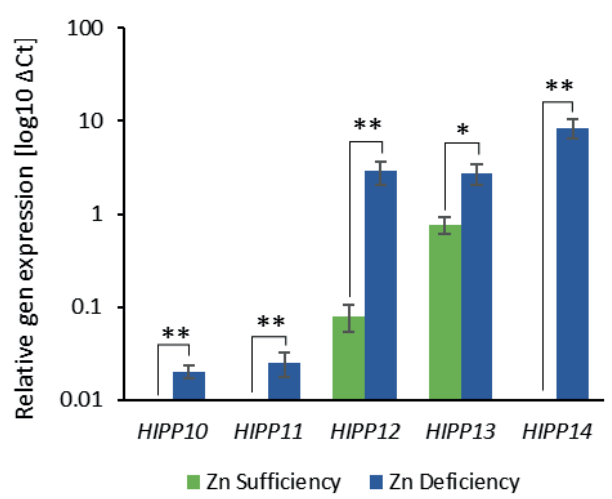

B

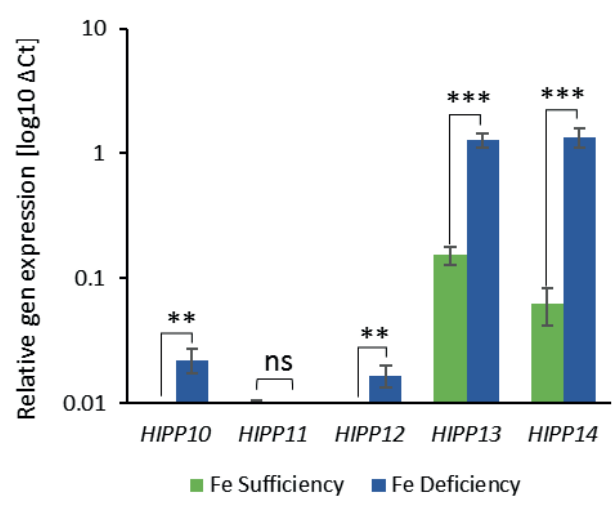

Figure 3. Expression of HIPP10, HIPP11, HIPP12, HIPP13 and HIPP14 genes is induced by Zn and Fe deficiency. HIPP transcription levels relative to the transcription of two stably transcribed reference genes (At5g25760 and At2g28390) are presented of (A) plants grown hydroponically under Zn sufficient (2 $\mu \mathrm{M} \mathrm{ZnSO}_{4}$ : green bars) or Zn-deficient (no added $\mathrm{Zn}$ : blue bars) conditions; and of (B) plants grown on vertical agar plates under $\mathrm{Fe}$ sufficient $\left(50 \mu \mathrm{M} \mathrm{Fe}(\mathrm{Na})_{2} \mathrm{EDTA}_{2}\right.$ : green bars) or Fe deficient (no added $\mathrm{Fe}$ : blue bars) medium. Mean $\pm S E, n=3$ replicates of 3 pooled plants each, for the $\mathrm{Zn}$ treatment, and $n=6$ replicates of $\sim 10$ pooled plants each, for the Fe treatment. Statistical significance was determined by Student's t test (ns $p>0.05, * p<0.05$, ** $p<0.01$, and $* * * P<0.001$ ).

\section{Disruption of individual HIPP genes affects the expression of several other HIPP genes under Fe deficiency}

To further investigate the function of the five HIPPs, we focused our research on the $\mathrm{Fe}$ deficiency, rather than the $\mathrm{Zn}$ deficiency response, due to the high tolerance to Fe deficiency of the hipp mutants (Figure 2B and C). We were most interested to understand why every single hipp mutant was similarly tolerant to Fe deficiency. Therefore, we determined the expression of the HIPP10, HIPP11, HIPP12, HIPP13, and HIPP14 genes in the four hipp mutants (hipp10, hipp 11, hipp12, and hipp 14) grown on Fe deficient medium. The expression of all genes but HIPP11, was induced by Fe deficiency in the wild type (Figure 3B). Under control conditions, the HIPP genes were expressed at comparable levels in the mutants as in the wild type (Figure 4), except for the genes which were mutated in the respective line (Supplemental Figure S1). There were a few additional exceptions, HIPP10 and HIPP12 were much higher expressed in the hipp 11 mutant than in the wild type, and HIPP 13 was lower expressed in the hipp 10 mutant. 
HIPP10

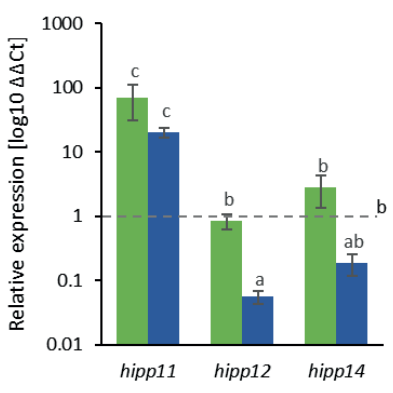

HIPP12

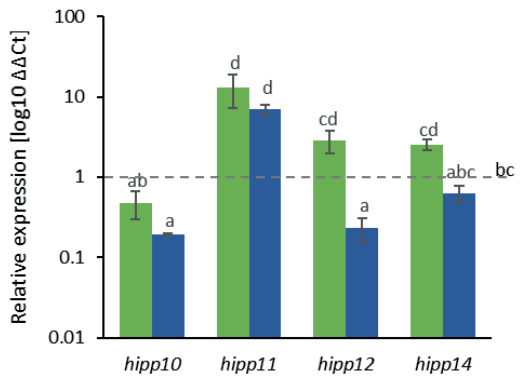

HIPP14

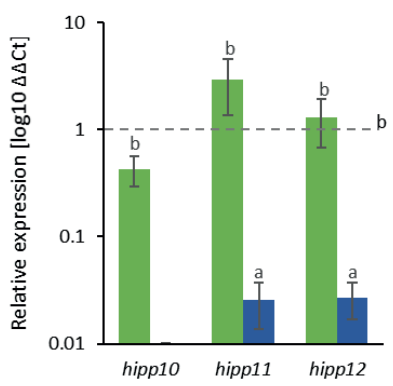

HIPP11

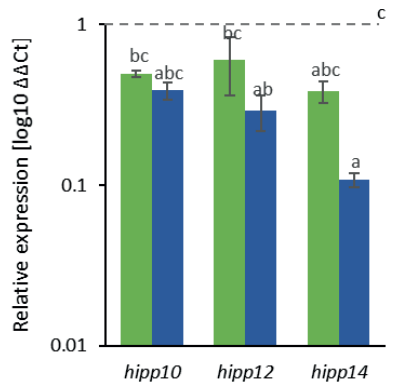

HIPP13

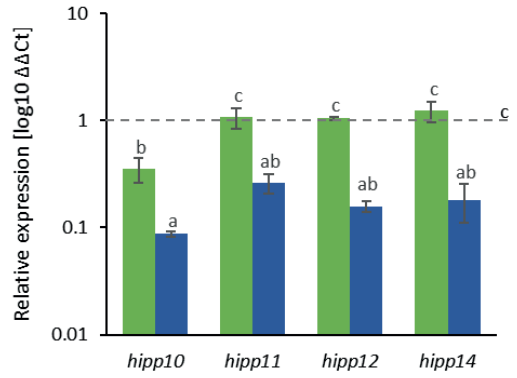

- Fe Sufficiency

- Fe Deficiency

Figure 4: Relative expression of HIPP10, HIPP11, HIPP12, HIPP13 and HIPP14 in shoots of hipp mutants grown in control conditions (Fe sufficiency) and under Fe deficiency. Transcript levels were normalized relative to reference genes At5g25760 and At2g28390 and to the expression in wild-type plants. Plants were grown on agar vertical plates under control conditions (medium containing $50 \mu \mathrm{M}$ $\mathrm{Fe}(\mathrm{Na})_{2} \mathrm{EDTA} A_{2}:$ green bars) or Fe deficiency (the same medium, without Fe added: blue bars). Mean \pm SE, $n=3$ replicates of $\sim 10$ pooled plants each. Letters above the bars denote statistically different groups, obtained with a Bonferroni post hoc test $(\alpha=0.05)$, after a one-way ANOVA $(p<0.001)$. The dashed line indicates the mean relative expression in wild type, and the letter at the end of the line indicates to which statistical group the wild type belongs. 
Under Fe deficiency, most HIPP genes are lower expressed in the hipp mutants compared to wild type, again with some exceptions, and often also lower expressed than under control conditions. HIPP10 and HIPP12 are again higher expressed in hipp 11 under Fe deficiency, and the expression of HIPP11 was not significantly different between hipp10 and wild type, nor was the expression of HIPP12 different between hipp14 and wild type. Taken together, disruption of individual HIPP genes did not only affect the expression of the mutated gene, but also that of several of the other HIPP genes, with the most prominent effects on expression of HIPP13 and HIPP14. This suggests that expression of HIPP genes may depend on HIPP interactions with transcription factors, which are different under Fe deficiency when compared to control conditions.

\section{The HIPP protein structures favour protein-protein interactions}

To infer a putative function of the HPPs and HIPPs encoded by the eleven genes in the cluster protein structures, active sites and biochemical functions were determined. Tehseen et al., (2010) predicted that HIPPs and HPPs contain HMA domain, using MotifScan (Pagni et al., 2007) we confirmed that five HIPPs and two HPPs are predicted to contain one HMA domain, while the remaining four HIPPs were predicted to have two HMA domains (Supplemental Figure S4). However, all HMA domains predicted in these proteins lack the M/LXCXXC core sequence of other known HMA domains (Tehseen et al., 2010). Therefore, rather than calling them HMA domains, we prefer to refer to them as HMA-like (HMAL) domains. Using Prenylation Prediction Suite (PrePS) (Maurer-Stroh and Eisenhaber, 2005) the C-terminal end of four HIPPs (HIPP10, 11, 13 and 14) was predicted to be the target of isoprenylation by farnesyltransferase (FT) or geranylgeranyltransferase 1 (GGT1), while HIPP12 was predicted to only be a target of FT. An isoprenylated C-terminus can mediate the attachment to other proteins or membranes (Konstantinopoulos et al., 2007). Using ANCHOR (Dosztányi et al., 2009), multiple disordered regions were detected in HPP9, HPP10, HIPP10, HIPP11, HIPP13 and HIPP14. Only HIPP12 was not predicted to contain disordered regions. Disordered regions also contribute to protein-protein interactions due to the flexibility they provide to the protein structure (van der Lee et al., 2014). 


\section{HIPPs interact with nuclear-localized proteins}

Given the potential of HIPPs to suppress plant growth under Fe deficiency, to affect the expression of each other, and of the HIPP protein to favour protein-protein interactions, we performed a dedicated $\mathrm{Y} 2 \mathrm{H}$ analysis to identify HIPP-interacting transcription factors. This revealed several of such potentially interacting transcription factors (Table 1). Several of these transcription factors were found to interact with more than one HIPP (Table1 and Supplemental Figure S5).

Table 1. Transcription factors that interact with HIPPs in a yeast-two-hybrid assay. Positive interactions were scored after 3 to 5 days of growth with a threshold of at least 5 colonies per diploid yeast on selective media.

\begin{tabular}{|c|c|c|c|c|c|c|c|}
\hline HIPP10 & HIPP11 & HIPP12 & HIPP13 & HIPP14 & $\begin{array}{c}\text { Locus } \\
\text { identifier }\end{array}$ & Symbol & Description \\
\hline yes & & & & & Atlg01010 & NAC001 & \\
\hline yes & & & & & At1g32870 & NAC13 & Induced by high levels of B \\
\hline yes & & & & & At1g34180 & NAC016 & Induced by salt and oxidative stress \\
\hline yes & & & & & At2g27300 & NTL8 & Induced by salt stress \\
\hline yes & & & & & At4g01550 & NAC69 & Mutants are resistance to salt \\
\hline yes & & & & & At4g35580 & NTL9 & Induced by osmotic stress \\
\hline yes & & & & & At5g22290 & NAC89 & Promotes cell death \\
\hline yes & & & yes & yes & Atlg15720 & TRFL5 & \\
\hline yes & & & yes & yes & At1g54060 & ASIL1 & Repressing seed maturation \\
\hline \multirow[t]{20}{*}{ yes } & & & yes & yes & At3g21270 & DOF2 & \\
\hline & yes & & & & At1g61660 & BHLH112 & Induced by salinity and drought \\
\hline & & yes & & & At2g02540 & HB21/ZFHD4 & \\
\hline & & yes & & & At2g20350 & ERF120 & Ethylene response factor \\
\hline & & yes & & & At3g25790 & $\mathrm{HHO}$ & Involved in $\mathrm{N}$ and $\mathrm{P}$ signaling \\
\hline & & yes & & & At3g49690 & MYB84/RAX3 & Regulates meristem formation \\
\hline & & yes & yes & & At5g41410 & BEL1 & Involved in ovule morphogenesis \\
\hline & & yes & & yes & At2g20570 & GLK1 & Regulates photosynthesis \\
\hline & & yes & & yes & At5g44190 & GLK2 & Regulates photosynthesis \\
\hline & & yes & & yes & At5g60142 & & \\
\hline & & & yes & & At3g04420 & NAC48 & \\
\hline & & & yes & yes & At2g28510 & DOF2.1 & \\
\hline & & & yes & yes & At3g11100 & ENAP1/VFP3 & Involved in ethylene signaling \\
\hline & & & yes & yes & At3g04930 & & \\
\hline & & & yes & yes & At3g54390 & & \\
\hline & & & yes & yes & At3g58630 & & \\
\hline & & & yes & yes & At5g05550 & ENAP2/VFP5 & Interacts with EIN2 \\
\hline & & & & yes & At1g59810 & AGL50 & \\
\hline & & & & yes & At3g45610 & DOF6 & Negative regulator of germination \\
\hline & & & & yes & At4g13480 & MYB79 & \\
\hline
\end{tabular}



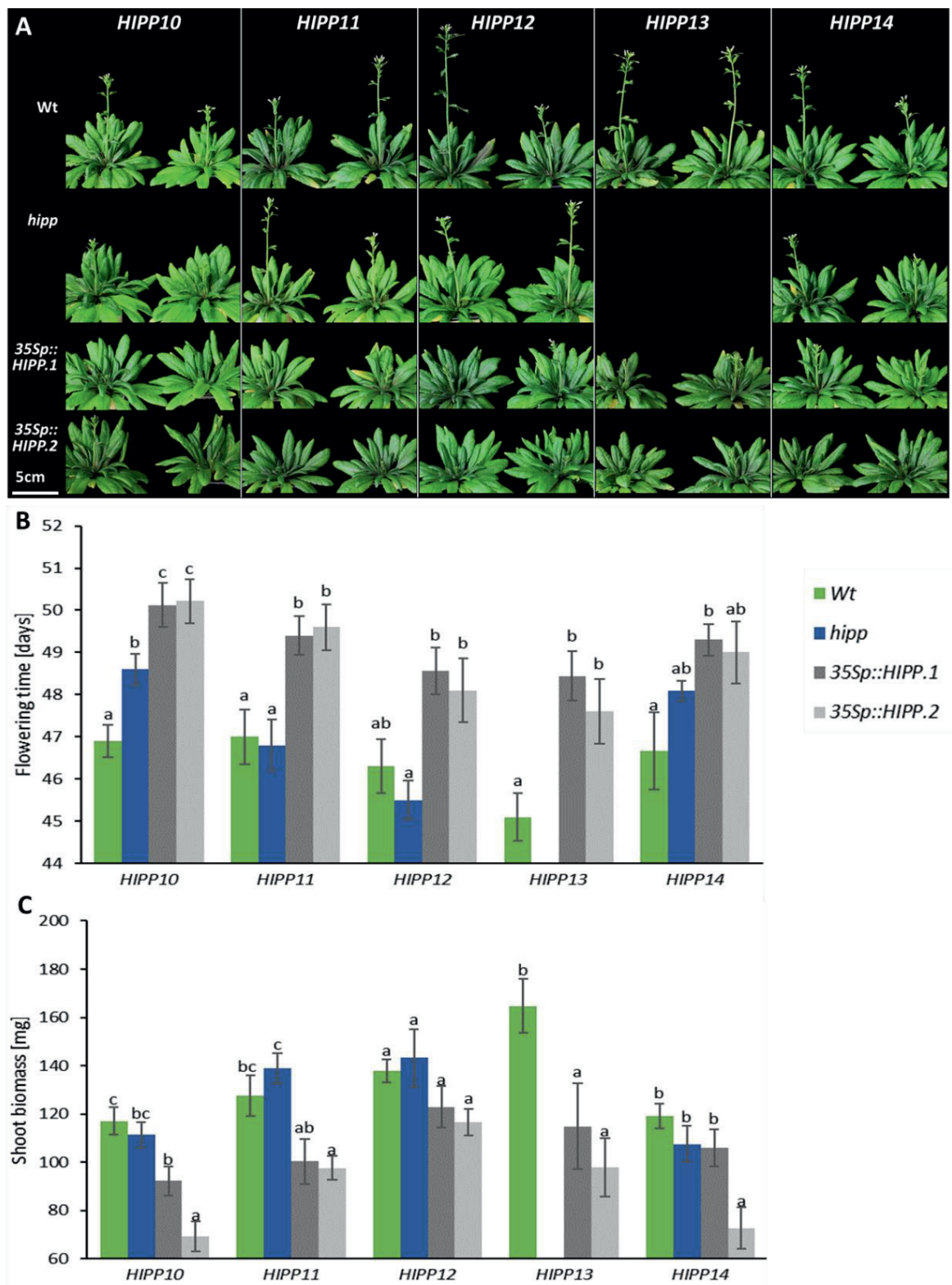

Figure 5. Phenotypes of hipp mutants and 35S-mediated HIPP overexpression lines grown until flowering upon sufficient Fe supply. (A) Plants grown for 48 days in a climate controlled greenhouse set at a $12 \mathrm{~h} \mathrm{light/dark} \mathrm{regime.} \mathrm{(B)} \mathrm{Flowering} \mathrm{time} \mathrm{(C)} \mathrm{biomass} \mathrm{of} \mathrm{wild} \mathrm{type} \mathrm{(Wt,} \mathrm{green} \mathrm{bars),} \mathrm{hipp} \mathrm{mutant}$ (blue bars), and 35Sp lines (35Sp::HIPP.1 or .2; dark and light grey bars) plants. Mean $\pm S E, n=5$ replicate plants per genotype. Letters above bars denote statistically different groups, comparing Wt, mutant and two 35Sp genotypes for each HIPP gene, grown together, as obtained with a Bonferroni post hoc test $(\alpha=0.05)$, after a one-way ANOVA $(p<0.001)$. 


\section{HIPPs affects flowering time}

Since the HIPP genes appear to act as negative regulators of plant growth in response to $\mathrm{Fe}$ and, to a lesser extent, Zn deficiency, we grew the hipp mutants and 35Sp::HIPP lines under Fe sufficient and mild Fe deficient conditions in the greenhouse to investigate any possible negative fitness trade-offs, especially under Fe deficiency. Next to the previously used 35Sp::HIPP10 lines, also 35Sp lines of HIPP11, HIPP12, HIPP13 and HIPP14 were generated and grown. All genotypes growing on Fe deficient medium developed chlorotic leaves, however, no genotype by treatment interaction effect was found for shoot biomass or flowering time. Seven of the ten 35Sp::HIPPs lines showed a consistent delay in flowering, and six on the ten $35 \mathrm{Sp}:: H I P P S$ lines showed less biomass compared to the wild type and hipp mutant lines, independent of the treatment (Figure 5).

\section{Discussion}

Plasticity is the physiological or morphological change of an organism in response to environmental clues (Schlichting, 1986). Stress conditions elicit a wide variety of plant responses, ranging from differences in gene expression to differences in growth and morphology. Environmental stress imposed on plants forces them to find a balance between growth and survival, which prompts some species or genotypes to invest in reproduction and quickly finish their life cycle, and others to cease growth and promote survival, often depending on the type and severity of the stress. Stress responses need to be tightly regulated due to antagonistic pleiotropic effects (Des Marais and Juenger, 2010; Todesco et al., 2010; Huot et al., 2014).

\section{Loss of function of each one of five HIPPs confers tolerance to unfavourable Fe and Zn supply}

The five HIPP genes studied here, were already described in Chapter 4, to reside at a quantitative locus on chromosome 5, associated with fluctuations in the root $\mathrm{Zn}$ concentration in response to $\mathrm{Zn}$ deficiency. The hipp10, hipp11 and hipp12 T-DNA insertion mutants were found to be more tolerant to $\mathrm{Zn}$ deficiency, in terms of biomass and higher $\mathrm{Zn}$ concentration in shoots. Here, we showed that single hipp10, hipp11, hipp12 and hipp14 mutants are not only more tolerant than wild types to Zn deficiency, but also to Zn excess, Fe excess and especially to Fe deficiency. The hpp 9 mutant also showed tolerance to excess of heavy metals (Sanz- 
Fernández et al., 2017), the HPP9 is part of the cluster of 11 tandemly arrayed HPP/HIPP genes located in chromosome 5 .

The homeostasis of both $\mathrm{Zn}$ and Fe are linked. Zn deficiency causes Fe concertation increase in shoots and decrease in roots (Chapter 4) and the $\mathrm{Zn}$ excess induces the expression $\mathrm{Fe}$ deficiency-responsive genes (van de Mortel et al., 2006) for instance. The most outstanding difference between the hipps mutants and wild type were detected upon Fe deficiency. The hipp10, hipp11, hipp12, and hipp14 mutant plants did not show any Fe deficiency symptom when growing on Fe deficient medium, plants were big and green as on Fe sufficient medium, while wild-type and 35Sp::HIPP10 plants grown on Fe deficient medium showed severe leaf chlorosis and inhibition of root and shoot growth, as is known to be the normal response to $\mathrm{Fe}$ deficiency (Varotto et al., 2002; Li et al., 2016). The decrease in growth due to stress is a common plant response to maximize survival, it is carried out by proteins involved in stressinduced growth modulation (Wolters and Jürgens, 2009). For instance, DELLA proteins integrate environmental and phytohormone signals, and repress plant growth under adverse conditions as salt stress (Achard et al., 2006).

An unexpected finding of this study was that single hipp mutants showed similar levels of tolerance to $\mathrm{Zn}$ deficiency, $\mathrm{Zn}$ excess and Fe deficiency. For instance, neither the single nor the double mutants for HIPP20, HIPP21, and HIPP22 exhibited any aberrant phenotype under any of the abiotic stress conditions tested, while only the hipp20/21/22 triple mutant was Cd sensitive (Tehseen et al., 2010). This suggests that despite their structural similarity, the HIPP10, HIPP11, HIPP12, and HIPP14 proteins are not functionally redundant, but instead appear to may have complementary functions in the Fe/Zn excess/deficiency stress response.

\section{HIPPs gene expression is determined by stress and auto-regulation}

Changes in gene expression are crucial for the organism adaptation to maximize survival under stress conditions (de Nadal et al., 2011). In eukaryote genomes, genes with similar expression may be clustered (Hurst et al., 2004). Here, we have shown that plants encountering limited $\mathrm{Zn}$ or Fe in their growth medium increase the expression of HIPP10, HIPP11, HIPP12, HIPP13, and HIPP14. Changes in gene expression in response to environmental clues are a common characteristic among Arabidopsis HIPP genes. Abiotic stresses caused by drought, salt, cold, exogenous ABA, and/or heavy metal affects the expression HIPP3 (Zschiesche et al., 2015), HPP9 (Sanz-Fernández et al., 2017), HIPP22, HIPP23, HIPP24, HIPP25, HIPP26, HIPP27 
and HIPP31 (Barth et al., 2009). The response deficiency of $\mathrm{Zn}$ or Fe can now be added to the list of environmental stresses that induce HIPPs expression.

The lower expression of the HIPP10, HIPP11, HIPP12, HIPP13 and HIPP14 genes in the hipp 10, hipp 11, hipp 12, and hipp14 mutants, in comparison to the wild type, particularly under Fe deficiency, suggests that these HIPP proteins, directly or indirectly, regulate the expression of the other genes in the HIPP gene cluster. This regulation is normally positive, meaning that HIPP expression enhances the expression of its neighbors, upon Fe deficiency, in what may be a positive feedback loop. Transcription regulatory feedback loops contribute to the efficiency of a transcriptional regulatory system, which may consist of a single component or may include several components and interactions among them (Mitrophanov and Groisman, 2008). Such regulation is specially necessary to balance trade-offs between stress response and fitness (Kasuga et al., 1999; Miller et al., 2015). Genes involved in similar processes can be controlled in an auto-regulatory loop. Proteins which interact with each other are known to be coordinately regulated in a positive feedback loop (Gómez-Mena et al., 2005). This also seems to be the case of the HIPP10-14 gene cluster. The result of their action is the high tolerance to adverse $\mathrm{Zn}$ and Fe nutrient by the loss of only one of them. We have not investigated the loss of several of these, which is not trivial to do, as it will require multiple CRISPR-Cas9-mediated mutations. Due to their close physical linkage, the regular way to generate double, triple or higher order combined mutations, by crossing single mutants, will not be practically feasible.

\section{HIPPs domains promote interactions}

HIPPs are classified as metallochaperone-like proteins due to their predicted HMA domain. The functional core motif of the HMA domain is characterized by CysXXCys or CysCysXXGlu (Tehseen et al., 2010). Some HIPPs containing this motif and experimentally confirmed to bind metals are HIPP26, which binds Pb, $\mathrm{Cd}$ and $\mathrm{Cu}$ (Gao et al., 2009); HIPP7, which binds $\mathrm{Cu}, \mathrm{Ni}$, or Zn (Dykema et al., 1999); and HIPP3, which binds Zn, Ni, Cu, and Fe (Achard et al., 2006). The HMA domain could be an allosteric switch that promotes changes in protein structure, as a response to stress, related with cellular location or binding affinity with different interactors. The resulting allosteric change upon binding a metal ion can affect the interaction with other proteins, such as transcription factors, and thus regulate gene expression (Guerra and Giedroc, 2012). For the mentioned HIPPs containing a functional HMA domain, enhanced or reduced binding to metals may be the way these HIPPs exert their function in response to heavy metal stress. 
However, out of 67 metallochaperone-like proteins in Arabidopsis, 45 HIPPs and 22 HPPs, 23 lack the functional core motif of the HMA domain (Tehseen et al., 2010). Among these are the six HPP and five HIPP proteins encoded by the HPP/HIPP gene cluster on chromosome 5 (Tehseen et al., 2010). Therefore, it is not clear if the predicted HMA-like (HMAL) domain of these eleven proteins is able to bind metals. We have not examined this any further, as it is not at all trivial, but considering their role in $\mathrm{Zn}$ or Fe deficiency response, it will be important for further understanding of their function and mode of action, to verify at least their ability to bind $\mathrm{Zn}$ or Fe. It could well be of course that this HMAL domain has taken a different evolutionary trajectory and acquired a new function. Significant changes in domain architecture are frequently observed upon tandem gene duplication (Buljan and Bateman, 2009; Jin et al., 2009), which is likely to be a reason of their remarkable clustering, as is evident from their phylogenetic relationship (Tehseen et al., 2010).

All HIPP sequences contain a C-terminal tetrapeptide recognition motif called the CAAX box. This box serves as a substrate for isoprenylation (Tehseen et al., 2010), which is the attachment of an isoprenoid lipid to the C-terminus (Scott Reid et al., 2004; Konstantinopoulos et al., 2007). After isoprenylation, the C-terminus becomes a hydrophobic domain, which mediates the attachment to other proteins or membranes (Konstantinopoulos et al., 2007). Isoprenylation is most prominent for proteins involved in signal transduction pathways (Roskoski, 2003). For instance, the isoprenylated C-terminus of HIPP26 interacts with ACYL-COA-BINDING PROTEIN 2 (ACBP2), which is associated with post-stress membrane repair and itself interacts with an ethylene-responsive element-binding protein (EBP), mediating stress response ( $\mathrm{Li}$ and Chye, 2004; Gao et al., 2009), through interaction with DELLA proteins (la Rosa et al., 2014).

Additionally, several intrinsically disordered regions (IDRs) were predicted in the HIPP10-14 sequences. These regions lack the hydrophobic core of structured regions and favour proteinprotein interactions, as flexible linkers between structured regions, specially required for the assembly of multiprotein complexes. Proteins with IDRs are common in protein interaction networks, acting as interaction hubs (Dunker et al., 2001; Fong et al., 2009; van der Lee et al., 2014). The plasticity that IDRs provide to proteins allows them to play key roles in cell signalling and regulation mechanisms (Wright and Dyson, 1999; Uversky et al., 2000; Iakoucheva et al., 2002; Galea et al., 2008; Van Roey et al., 2012). In addition, IDRs at the Cterminus are associated with transcription factor repressors, while internal or N-terminus IDR are associated with transcription factor activators (van der Lee et al., 2014). HIPP10, 11, 13 and 
14 contain predicted internal and C-terminus IDRs, thus a hypothetical activation or repression activity is not clear. The isoprenylation motif and the IDRs suggest the ability of HIPP10-14 to interact with other proteins with the potential to link different pathways.

\section{The HIPP10-14 proteins interact with multiple nuclear-localized proteins}

Protein interacting partners provide clues about the pathways in which unknown proteins are involved. All proteins encoded by the HIPP10-14 cluster showed interaction with several transcription factors. Therefore, HIPPs seem to be highly connected proteins which could work as hubs of protein complexes to interconnect pathways, as is observed for APETALA1 (AP1) and FRUITFULL (FUL) involved in floral meristem identity and floral organ determination (de Folter et al., 2005).

HIPP10 showed interaction with seven NAM, ATAF1/2, and CUC2 (NAC) proteins, which have regulatory functions in plant growth, development, and stress responses ( $\mathrm{Li}$ et al., 2010; Nakashima et al., 2012). Among them NAC13 is induced by high B concentrations (Kasajima and Fujiwara, 2007), and is involved in the cross-talk between ROS signaling (De Clercq et al., 2013), hormone signaling and plant development (O'Shea et al., 2015). Salt and oxidative stress promote NAC16 gene expression. The nac16 mutant stays green under salt and oxidative stress, while overexpression NAC16 senesced rapidly (Kim et al., 2013). NAC16 induces the transcription of STAYGREEN1 (SGR1), which promotes chlorophyll degradation during abiotic stress or plant senescence (Sakuraba et al., 2016). NAC69 integrates salt and auxin signals during germination and nac69 mutants are resistant to salt stress (He et al., 2015). NAC89 positively regulates programmed cell death, while it negatively regulates chloroplast antioxidant defence system (Klein et al., 2012). NTM1-Like 8 (NTL8) is induced by salinity, the ntl 8 mutant seeds are resistant to high salinity during germination (Kim et al., 2008) and the overexpression of NTL 8 shows reduced growth and delayed flowering (Kim et al., 2007). NTL9 is induced by osmotic stress and mediates osmotic stress signaling during senescence (Kim et al., 2007). The potential interactors of HIPP10 have as a common pattern to be induced by stress conditions such as high concentration of boron, salinity and osmotic stress, their overexpression promotes senescence or reduces growth and their loss of function promotes tolerance to the stress that induces their expression. The HIPP10-14 have a similar behaviour, as these interactors, being induced by the stress conditions of $\mathrm{Zn}$ and Fe deficiency, their overexpression reduces growth, and their loss of function promotes tolerance to $\mathrm{Zn}$ and Fe deficiency. The 
difference between the HIPP10 interactors and HIPP10-14 is the stress factor studied that triggers the response.

For HIPP11, we found one interaction with bHLH112, a transcription factor induced by salinity, drought, and ABA. It positively regulates salt and drought tolerance (Liu et al., 2015). Among the HIPP12 interactors, ZFHD4 is highly expressed in floral tissues (Tan and Irish, 2006) and also interacts with HIPP26 (Barth et al., 2009). HHO1 represses primary roots growth under P deficiency, its mutants are insensitive to P deficiency (Medici et al., 2015). RAX3 regulates axillary meristem initiation (Müller et al., 2006). HIPP12 and HIPP14 interact with GLK1 and GLK2, which coordinate the expression of photosynthetic genes to regulate chloroplast development and optimize photosynthesis (Fitter et al., 2002; Waters et al., 2009; Murmu et al., 2014). Some proteins that showed interaction with more than one HIPP of the cluster also interact with other HIPPs. Among them are ASIL1 which also interacts with HIPP3 (Zschiesche et al., 2015), HIPP21 and HIPP27 (Provart et al., 2016); DOF2 also interacts with HIPP26 (Provart et al., 2016); and AT3G54390 interacts with HIPP20 as well (Provart et al., 2016).

HIPP13 and HIPP14 have six common interactors, among them, ENAP1 inhibits the transcription of ethylene-repressed genes in the presence of ethylene. Seedlings overexpressing of ENAP1 are hypertensive to ethylene and smaller than wild type, while the down-regulation of ENAP1 makes plants less sensitive to the ethylene with even bigger size than wild type (Zhang et al., 2016; Zhang et al., 2017; Zhang et al., 2018). Ethylene is produced by Fe deficient plants and regulates the expression of several Fe acquisition genes (Lucena et al., 2006; García et al., 2010; Liu et al., 2017).

The hypothesis of complementary functions among HIPP10-14 is supported by the presence of common interactors among them (Supplemental Figure S5). The functions of the HIPPs interactors reinforce the responses observed for the HIPP10-14 genes in relation to induction by stress, stress tolerance and late flowering and give hints to study the relation of ethylene and salt with the HIPPs cluster. In addition, all the interactions detected in a $\mathrm{Y} 2 \mathrm{H}$ assay should be confirmed by in vivo FRET-FLIM (Long et al., 2017) assays.

\section{Flowering time}

The overexpression of HIPP10-14 resulted in a delay of flowering (Figure 5). In addition, the overexpression of the HIPPs interactors NTL8, NAC89, and ARABIDOPSIS 6B- 
INTERACTING PROTEIN 1-LIKE 1 (ASIL1) also showed delayed flowering (Kim et al., 2007; Li et al., 2010; Park et al., 2011). Furthermore, HIPP12 and HIPP13 interact with BEL1, which is important to maintain the indeterminacy of the inflorescence meristems (Bellaoui et al., 2001). Thus the action of the HIPP10-14 proteins may also involve the indirect control of flowering time, upon Fe and $\mathrm{Zn}$ deficiency, as part of the plant survival mechanism. Flowering is known to be regulated by internal and environmental stimuli to ensure plant reproduction under the most favourable conditions. Biotic and abiotic stress factors play a role in the transition to flowering (Riboni et al., 2014; Kazan and Lyons, 2016). For instance, drought stress accelerates the flowering process (Franks, 2011; Schmalenbach et al., 2014), while salt stress delays flowering time (Kim et al., 2013). Nutrient availability, including Zn and Fe supply, also regulates the onset of flowering (Sivitz et al., 2012; Przedpelska-Wasowicz and Wasowicz, 2013; Lin and Tsay, 2017; Chen and Ludewig, 2018; Weber and Burow, 2018).

\section{Conclusions}

Our findings reveal that HIPPs are part of the $\mathrm{Zn}$ and Fe deficiency response. The mutation of HIPP10, 11, 12 or 14 generates a similar level of tolerance to $\mathrm{Zn}$ and Fe deficiency and their expression is induced by $\mathrm{Zn}$ and Fe deficiency. Moreover, the constitutive expression of these genes represses growth and delays flowering time. It places the HIPP10-14 genes in pathways connecting $\mathrm{Zn} / \mathrm{Fe}$ deficiency stress with plant growth and development. In these pathways, HIPPs can interact directly among them or through common interaction partners. Further research is required to understand the role of HIPP10-14 in mediating stress-induced growth inhibition for stress tolerance. These genes can be useful candidates to engineer stress tolerant crops, considering that we did not find any trade-offs in the hipps mutants.

\section{Acknowledgements}

We thank Prof. Richard Immink and Marco Busscher (Wageningen University) for facilitating the $\mathrm{Y} 2 \mathrm{H}$ cDNA library and the technical support in the $\mathrm{Y} 2 \mathrm{H}$ assay respectively. This work was supported by the Ecuadorian government through SENESCYT (Secretaría de Educación Superior, Ciencia, Tecnología e Innovación) and Universidad de las Fuerzas Armadas-ESPE. 


\section{Supplemental information}

Table S1. Sequences of primers used for quantification of gene expression by $q R T-P C R$

\begin{tabular}{ccll}
\hline $\begin{array}{c}\text { Locus } \\
\text { identifier }\end{array}$ & Gene & Primer & 5'->3' sequence \\
\hline At5g52720 & HIPP10 & Forward & GCAGGAGACGGTTGTATTCG \\
At5g52720 & HIPP10 & Reverse & CCTCTTTCCTTCACGTCTATCAC \\
At5g52730 & HIPP11 & Forward & GTTACAAGGCGTGAACCCAA \\
At5g52730 & HIPP11 & Reverse & GAGGTATGGACGGACGAGAG \\
At5g52740 & HIPP12 & Forward & TCGGTGGAGGTGAAAGATGG \\
At5g52740 & HIPP12 & Reverse & TCGTTTCAGGCTTCTTCGGA \\
At5g52750 & HIPP13 & Forward & TGAACCAGAGAAACCGGCTC \\
At5g52750 & HIPP13 & Reverse & GGCAGGATTGTATTGGTAAGGG \\
At5g52760 & HIPP14 & Forward & TGTACCAGCTGTCGTGATGA \\
At5g52760 & HIPP14 & Reverse & GGTACGCGTAGTTCATCGGA \\
At2g28390 & SAND & Forward & GTTGGGTCACACCAGATTTTG \\
At2g28390 & SAND & Reverse & GCTCCTTGCAAGAACACTTCA \\
At5g25760 & PEX4 & Forward & TCCTGAGCCGGACAGTCCTC \\
At5g25760 & PEX4 & Reverse & CATAGCGGCGAGGCGTGTAT \\
\hline
\end{tabular}

Table S2. Sequences of primers used to clone HIPP gene coding sequences from cDNA. Forward primers include the attB1 linker (GGGGACAAGTTTGTACAAAAAAGCAGGCTTA) and reverse primers include the attB2 linker (GGGGACCACTTTGTACAAGAAAGCTGGGTA) at their 5' ends. The ATG start codons are underlined.

\begin{tabular}{clll}
\hline Locus identifier & Gene & Primer & 5'->3' sequence \\
\hline At5g52720 & HIPP10 & Forward & $\underline{\text { ATGCAGGAGACGGTTGTATTC }}$ \\
At5g52720 & HIPP10 & Reverse & TCACAGGATGATACACTCATCCG \\
At5g52730 & HIPP11 & Forward & CGGATGTATATAAATACACATGACAGT \\
At5g52730 & HIPP11 & Reverse & TTACATGATGATACAACCATCCG \\
At5g52740 & HIPP12 & Forward & ATGCAGGTAGTTGTACTGAAATTAGAT \\
At5g52740 & HIPP12 & Reverse & TCAAGAGGTGACGCAAGC \\
At5g52750 & HIPP13 & Forward & ATGCCTCCAATGAAAGCTGT \\
At5g52750 & HIPP13 & Reverse & TCACATAATCACACAATTTGATTCG \\
At5g52760 & HIPP14 & Forward & ATGACCGCAAAGAACGCT \\
At5g52760 & HIPP14 & Reverse & TCACATAATCACACAATTTGGTTC \\
\hline
\end{tabular}


Table S3. One-way ANOVA of projected leaf area of wild-type, hipp mutants and 35Sp::HIPP10 plants, grown under Zn deficient, Zn excess, Fe deficient and Fe excess conditions, and compared to control conditions. Significant effects are indicated in bold.

\begin{tabular}{|c|c|c|c|}
\hline Treatment & Genotype & Variance ratio & F probability \\
\hline Zn deficiency & Wild type & 17.93 & $<.001$ \\
\hline Zn deficiency & hipp10 & 1.39 & 0.266 \\
\hline Zn deficiency & hippl1 & 6.11 & 0.033 \\
\hline Zn deficiency & hipp12 & 2.12 & 0.176 \\
\hline Zn deficiency & hipp14 & 0.21 & 0.655 \\
\hline Zn deficiency & 35Sp::hipp10.1 & 0.03 & 0.865 \\
\hline Zn deficiency & 35Sp::hipp 10.2 & 1.9 & 0.198 \\
\hline Zn excess & Wild type & 29.28 & $<.001$ \\
\hline Zn excess & hipp10 & 0.01 & 0.927 \\
\hline Zn excess & hippl1 & 27.63 & $<.001$ \\
\hline Zn excess & hipp12 & 6.93 & 0.025 \\
\hline Zn excess & hipp14 & 0.06 & 0.817 \\
\hline Zn excess & 35Sp::hipp10.1 & 6.76 & 0.027 \\
\hline Zn excess & 35Sp::hipp10.2 & 14.88 & 0.003 \\
\hline Fe deficiency & Wild type & 134.53 & $<.001$ \\
\hline Fe deficiency & hipp10 & 3.15 & 0.106 \\
\hline Fe deficiency & hippl1 & 1.63 & 0.23 \\
\hline Fe deficiency & hipp12 & 0.54 & 0.48 \\
\hline Fe deficiency & hipp14 & 0.54 & 0.48 \\
\hline Fe deficiency & 35Sp::hipp10.1 & 92.64 & $<.001$ \\
\hline Fe deficiency & 35Sp::hipp10.2 & 234.85 & $<.001$ \\
\hline Fe excess & Wild type & 137.33 & $<.001$ \\
\hline Fe excess & hipp10 & 50.44 & $<.001$ \\
\hline $\mathrm{Fe}$ excess & hippl1 & 17.27 & 0.002 \\
\hline $\mathrm{Fe}$ excess & hipp12 & 106.22 & $<.001$ \\
\hline Fe excess & hipp14 & 106.22 & $<.001$ \\
\hline Fe excess & 35Sp::hipp10.1 & 84.64 & $<.001$ \\
\hline Fe excess & 35Sp::hipp10.2 & 73.96 & $<.001$ \\
\hline
\end{tabular}



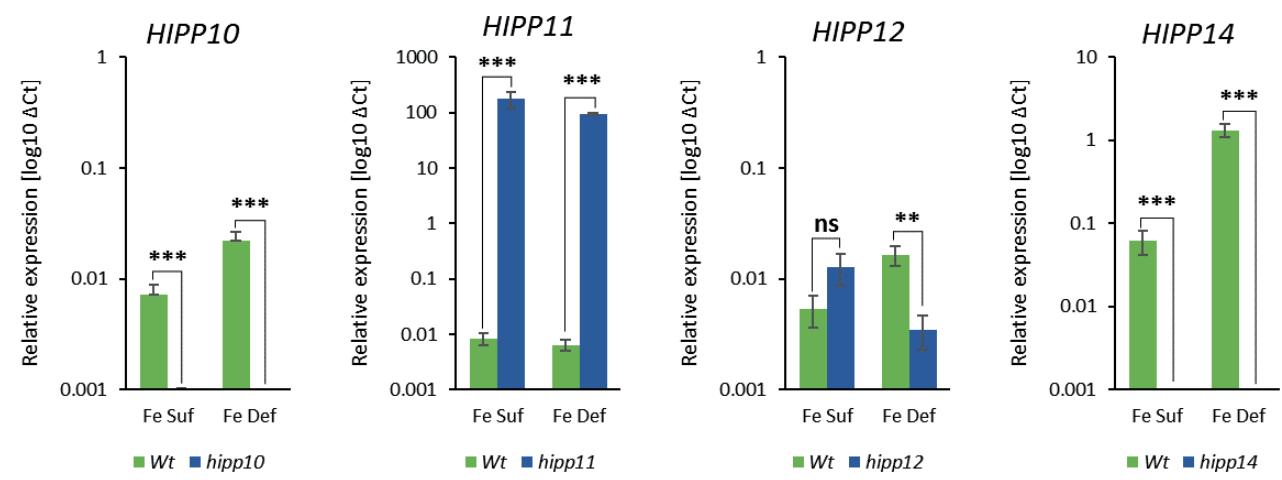

Figure S1. Expression of HIPP10, HIPP11, HIPP12, and HIPP14 genes is their respective T-DNA insertion lines. HIPP transcription levels relative to the transcription of two stably transcribed reference genes (At5g25760 and At2g28390) are presented of wild-type plants (green bars) and hipp mutants (blue bars) grown on vertical agar plates under Fe sufficient $\left(50 \mu \mathrm{M} \mathrm{Fe}(\mathrm{Na})_{2} \mathrm{EDTA}_{2}\right.$ : $\mathrm{Fe} \mathrm{Suf}$ ) or $\mathrm{Fe}$ deficient (no added Fe: Fe Def) medium. Mean $\pm S E$, and $n=6$ replicates of $\sim 10$ pooled plants each, for the Fe treatment. Statistical significance was determined by Student's t test ( $* * p<0.01$, and *** $P<0.001)$. 
Chapter 5
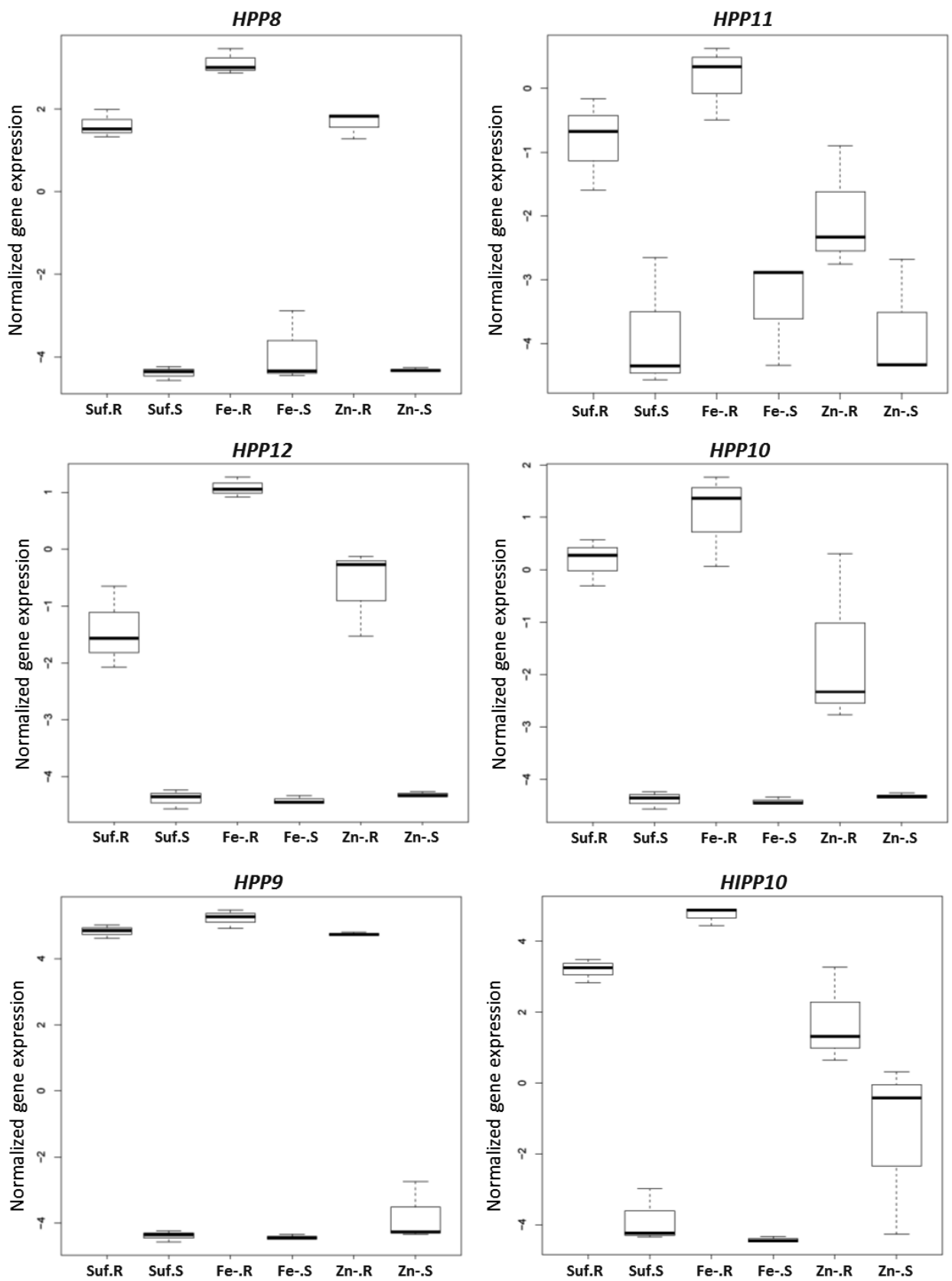

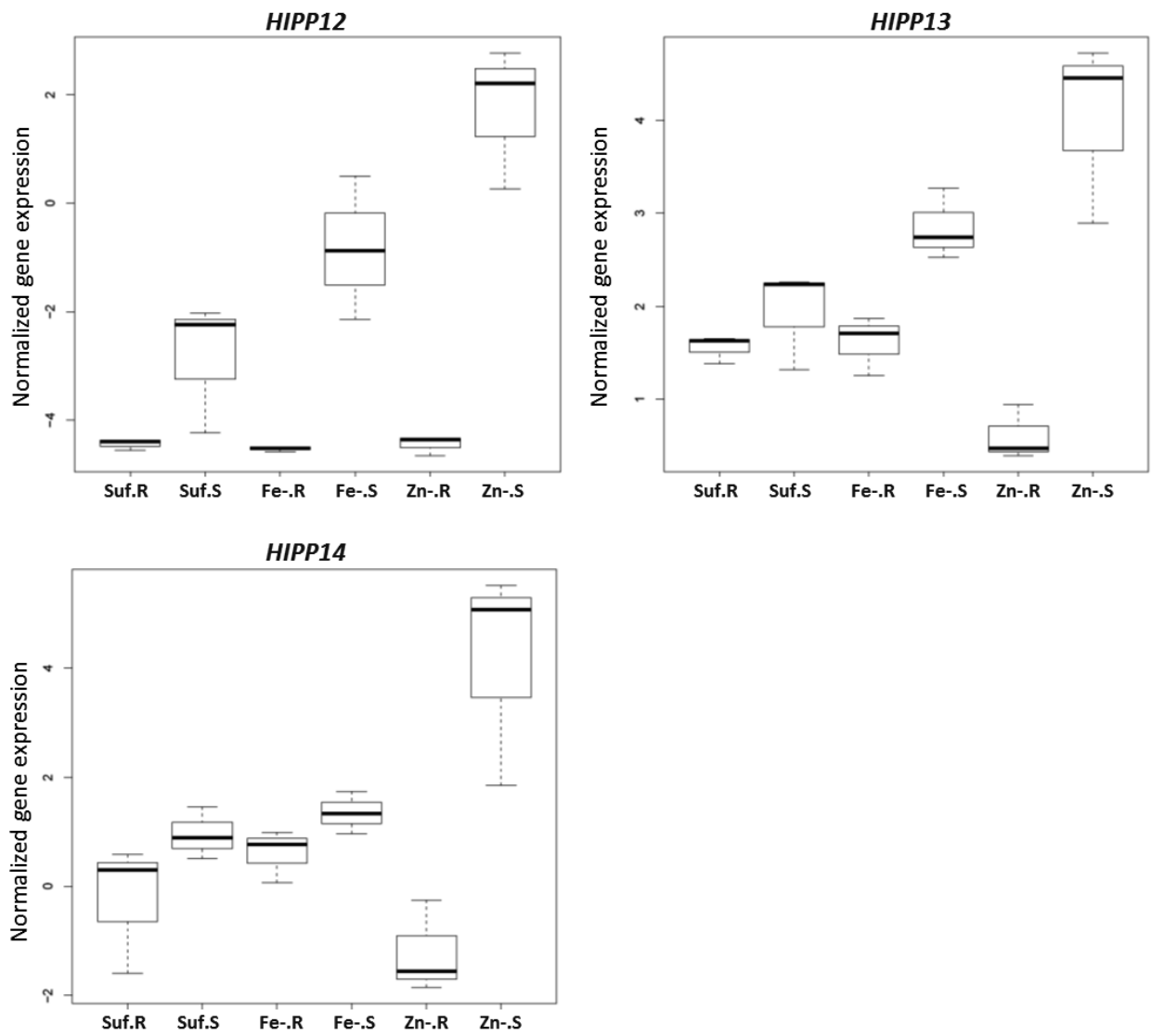

Figure S2. Expression of HPP and HIPP genes in shoots $(S)$ and roots $(R)$ of wild-type plants grown on control (Suf), Zn deficient (Zn) and Fe deficient (Fe) medium. Data from RNA-seq reads (Chapter 3). 
A

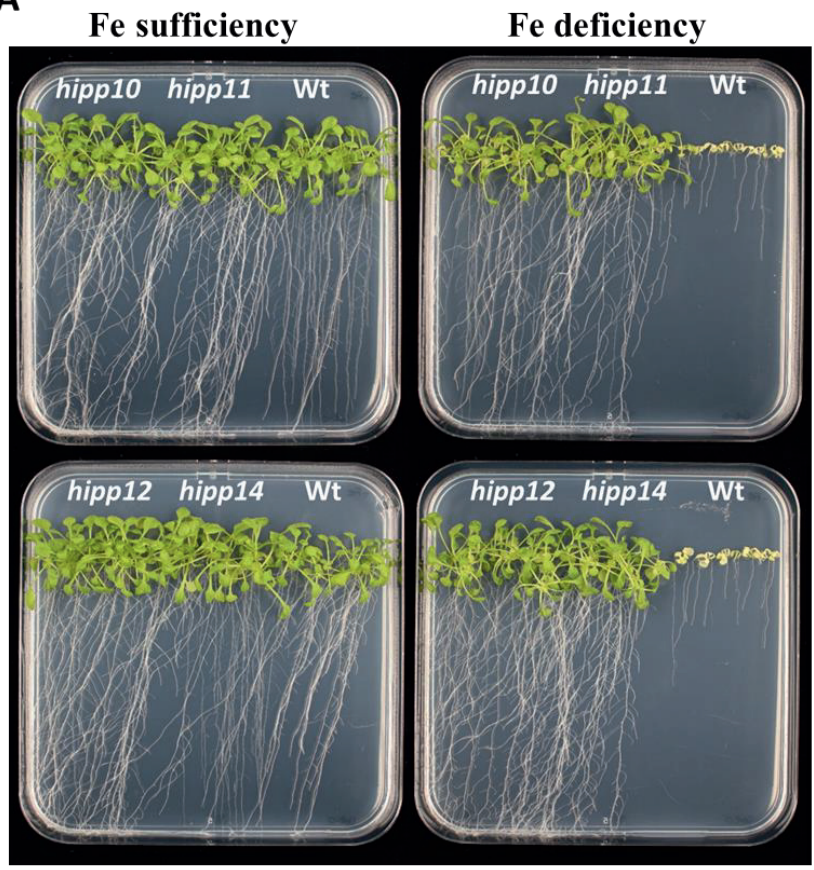

B

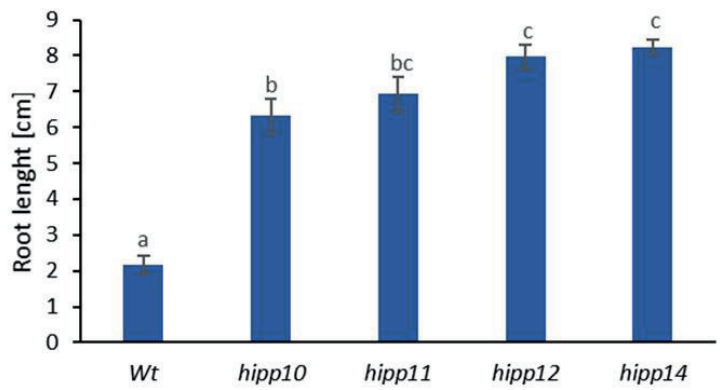

Figure S3. hipp mutants have increased tolerance to Fe deficiency. (A) Wild-type, hipp and 35Sp::HIPP10 plants grown for three weeks on control $\left(50 \mu \mathrm{M} \mathrm{Fe}(\mathrm{Na})_{2} E D T A_{2}\right)$ and Fe-deficient (no added $\mathrm{Fe}$ ) medium. (B) Root lengths of wild-type and hipp mutant plants grown on Fe-deficient medium. Mean $\pm S E, n=22$. Letters above the bars denote statistically different groups when comparing genotypes, obtained with a Bonferroni post hoc test $(\alpha=0.05)$, after a one-way ANOVA $(p<0.001)$. 


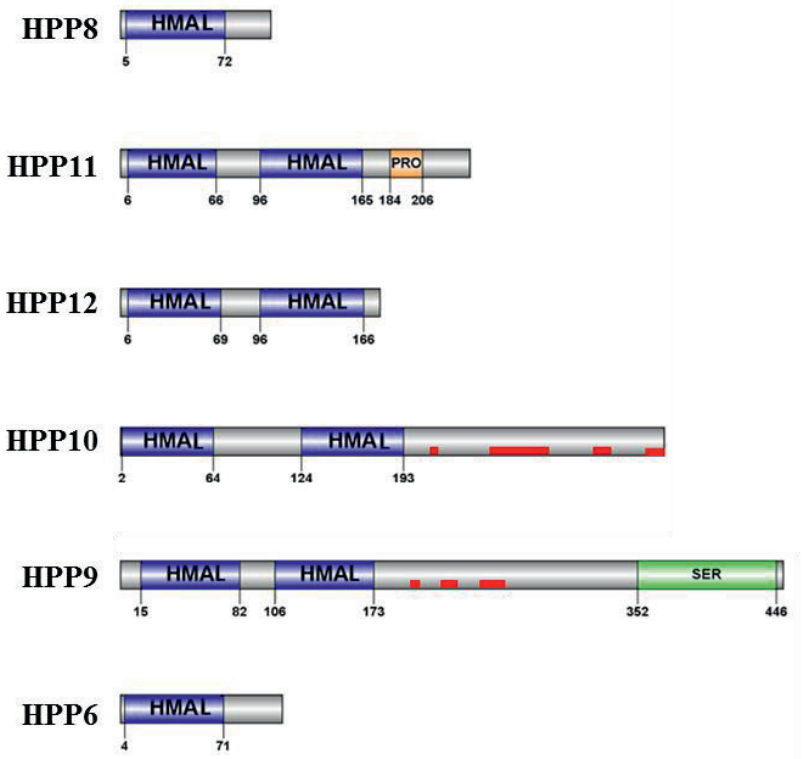

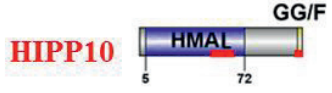
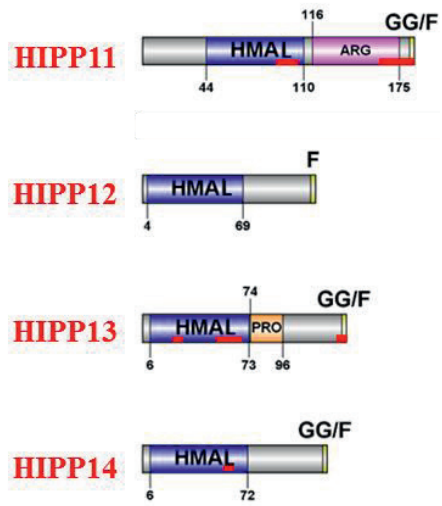

Figure S4. Annotated domains and regions in the proteins encoded by the eleven clustered HPP and HIPP genes. Amino acid positions are indicated by the numbers below the bars. HMA (blue) = Heavy Metal Associated domain; $P R O($ orange $)=$ proline-rich region; $S E R$ (green) = serine-rich region; $A R G$ $($ pink $)=$ arginine-rich region; $F$ (yellow) = farnesylation motif; $G G$ (yellow) = geranylgeranylation motif. Disordered regions are indicated in a separate (lower) bar in red. 


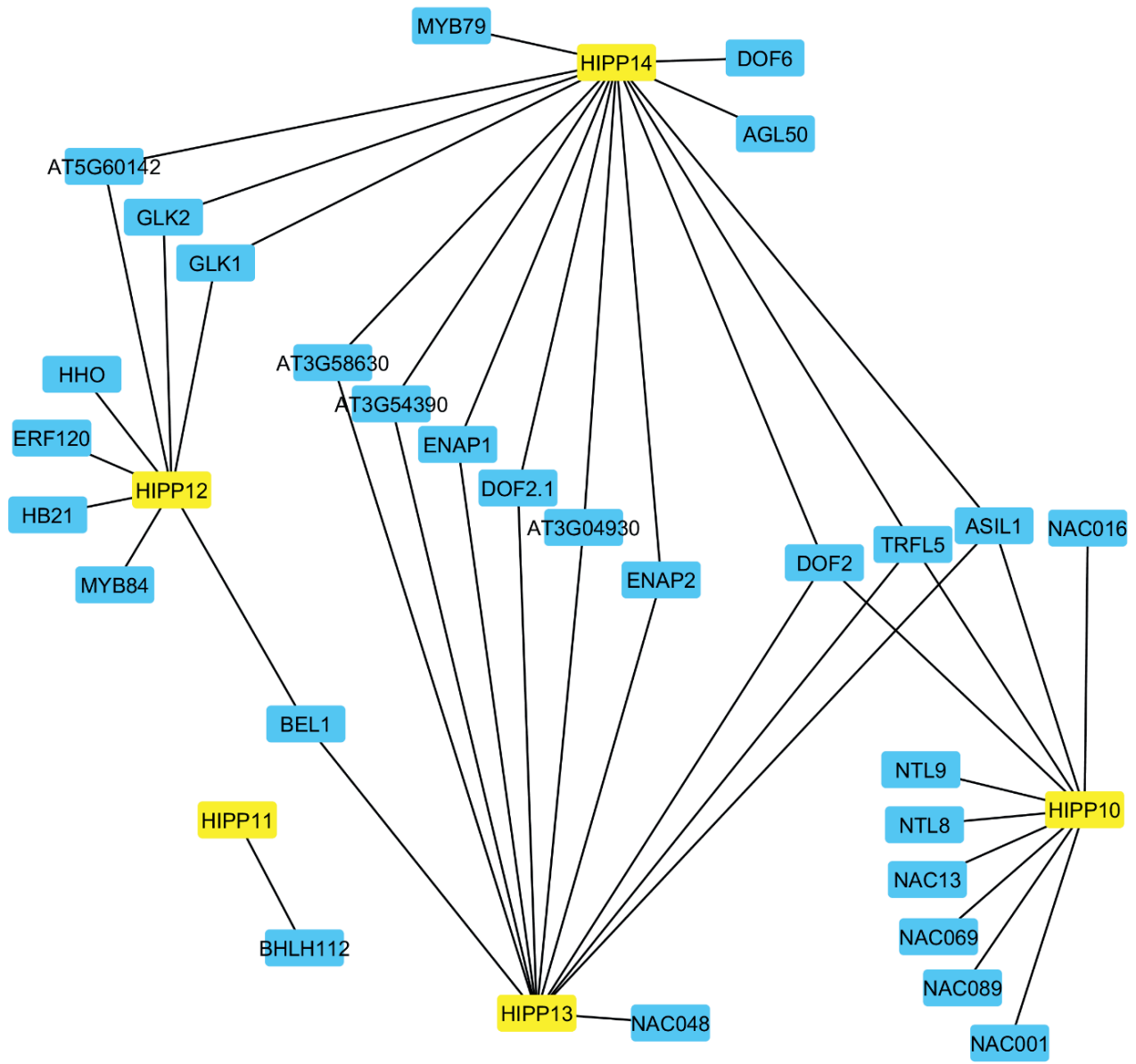

Figure S5. Network of HIPP10-14 and their interactors obtained by Y2H assay. Proteins are indicated by rectangles (HIPPs are yellow, and their interactors are blue) and protein interactions with lines. 



\section{Chapter 6}

General Discussion 


\section{Discussion}

\section{Introduction}

In my thesis, I have used forward and reverse genetics approaches to learn more about the genetic architecture of $\mathrm{Zn}$ homeostasis, especially under $\mathrm{Zn}$ deficiency conditions, in the model species Arabidopsis thaliana (Arabidopsis). The results obtained in my thesis research provide enlightening information about the $\mathrm{Zn}$ homeostasis, but also show all the challenges of untangling the genetic architecture of $\mathrm{Zn}$ homeostasis. $\mathrm{Zn}$ homeostasis requires the concert of several processes, molecules, and genes. This is the reason why the study of $\mathrm{Zn}$ homeostasis took me through different paths focusing on Zn transporters (Chapter 2), proteins modification as acetylation (Chapter 3), natural variation (Chapter 4), negative-growth regulators (Chapter 5) and homeostasis of other elements as Fe (Chapters 3, 4, and 5).

\section{Sneaky Zn transporters function}

The $\mathrm{Zn}$ homeostasis consists of tight control of the $\mathrm{Zn}$ cellular concentration to keep the proper physiological concentrations avoiding toxicity or deficiency, regardless of Zn supply (Krężel and Maret, 2006; Sinclair and Kräm er, 2012; Krężel and Maret, 2016) Along the years of study of Zn homeostasis, Zn transporters have been the 'stars' of this process (Eide, 2006), even more players are involved in it. To understand better the $\mathrm{Zn}$ homeostasis, we can start to disentangle it in terms of an isolated cell. Firstly, the cell should contain genes that codify $\mathrm{Zn}$ transporters able to scavenge $\mathrm{Zn}$ from the surroundings and transport it into the cell, exclude excess $\mathrm{Zn}$ from the symplast of the cell, or store Zn into cellular compartments (Regalla and Lyons, 2006). Secondly, the expression of these transporters should be regulated by at least one protein able to sense $\mathrm{Zn}$ concentration in the cell (Bird et al., 2003). Thus, in this ideal unicellular system, the cell will need at least four genes driving the $\mathrm{Zn}$ homeostasis. However, even in a unicellular organism, such as yeast, the $\mathrm{Zn}$ homeostasis is not so simple. Yeast encodes for more than ten proteins directly involved in Zn homeostasis (Regalla and Lyons, 2006; Eide, 2009). Yeast was the organism, in which the first Zn transporter was described (Eide et al., 1996; Zhao and Eide, 1997).

When we move to a more complex organism like a plant, we will find organs, tissues, and cell layers. Each one of them with specialised functions, which are coordinated by cell-cell signalling (Zhu, 2016). For instances, upon exposure to high $\mathrm{Zn}$ concentrations, $\mathrm{Zn}$ is not sequestered in every single cell of the plant. High concentrations of $\mathrm{Zn}$ have been found in nonphotosynthetic tissues such as epidermal cells (Kuepper et al., 1999). One of the genes responsible for driving this selective $\mathrm{Zn}$ storage is METAL TRANSPORTER PROTEIN 1 
(MTP1), whose protein remobilizes $\mathrm{Zn}$ from the symplast into the vacuole (Dräger et al., 2004; Kobae et al., 2004; Gustin. et al., 2009). In another case, upon exposure to $\mathrm{Zn}$ deficiency conditions, the HEAVY METAL ATPASE 2 (HMA2) expresses in the vasculature to favour the $\mathrm{Zn}$ root to shoot translocation (Hussain et al., 2004; Sinclair et al., 2007). Therefore, in complex organisms apart from keeping the Zn equilibrium in only one cell, the processes need to be coordinated to keep the equilibrium in all cell types, which perform different functions. This level of complexity increases the degree of difficulty to find all genes involved in $\mathrm{Zn}$ homeostasis and discern their functions.

All the genes that we know to be involved in Zn deficiency in Arabidopsis come from studies either of gene homology with other organisms (Mäser et al., 2001), induction of gene expression due to Zn deficiency (Wintz et al., 2003; van de Mortel et al., 2006), or restoration of the normal growth of the Saccharomyces cerevisiae zrt1zrt2 mutant defective in Zn uptake (Grotz et al., 1998; Assunção et al., 2010; Milner et al., 2013). The results of these studies correlate with each other supporting the role of these genes in $\mathrm{Zn}$ transport.

Almost, after 20 years of the discovery of the first $\mathrm{Zn}$ transporter in Arabidopsis, there have not been reports showing an increase in $\mathrm{Zn}$ deficiency sensitivity due to the loss of function of any known $\mathrm{Zn}$ transporters induced by $\mathrm{Zn}$ deficiency. No specific $\mathrm{Zn}$ transporter stands out in genetic studies during the analyses of plant extreme phenotypes or during the screening of mutant populations. This suggests that there are redundancies among Arabidopsis $\mathrm{Zn}$ transporters and/or Zn transporters have small effects over the Zn deficiency phenotype.

Due to the lack of reports on this topic, I decided to fill this gap in knowledge by studying the $\mathrm{Zn}$ deficiency sensitivity in single $\mathrm{Zn}$ transporters loss of function mutants. As expected, the loss of function mutants of zip1, zip3, zip5, zip9, zip 12, irt3, and mtp2 did not show a significant increase in the sensitivity to Zn deficiency (Chapter 2) compared to wild-type plants. Still these genes have been shown to be able to transport Zn (Grotz et al., 1998; Assunção et al., 2010; Milner et al., 2013). The expression of these genes is induced after six hours of exposure to $\mathrm{Zn}$ deficiency in roots and after more than two days in shoots. However, mutations in them do not give an increased effect on the plant $\mathrm{Zn}$ deficiency sensitivity (Chapter 2). The lack of phenotype strongly suggests the presence of the functionally redundant genes (Pickett and Meeks-Wagner, 1995) involved in the $\mathrm{Zn}$ deficiency response. 


\section{Discussion}

Still encouraged by all the information supporting the $\mathrm{Zn}$ transport function of these genes, I continued to search for increased sensitivity to $\mathrm{Zn}$ deficiency. So, I generated several combinations of double mutants of these transporters. Most of the double mutant combinations did not show an increased sensitivity to $\mathrm{Zn}$ deficiency (Chapter 2). Thus, it could be that the genes selected for each combination are not redundant pairs or that there are more than two redundant genes able to fulfil the same function. Partial or total functional redundancy has been encountered when more than one gene needs to be mutated to observe a phenotype (Pickett and Meeks-Wagner, 1995). The metal transport proteins HMA2 and HMA4 show partial functional redundancy for Zn transport for example. Single hma2 and hma4 mutants exhibit no apparent phenotype, while the double mutant hma2/hma4 is highly sensitive to $\mathrm{Zn}$ deficiency (Hussain et al., 2004).

In the case of the $\mathrm{Zn}$ transporters, the double mutant combinations of zipl/zip3 and zip3/zip5 showed increased sensitivity to $\mathrm{Zn}$ deficiency. The double mutant zip3/zip5 showed the strongest sensitivity to $\mathrm{Zn}$ deficiency, which is a synergistic effect on the $\mathrm{Zn}$ deficiency phenotype considering the lack of phenotype of the single mutants. Their coding sequences are highly similar (Mäser et al., 2001) and both genes have similar induction timing and location patterns of expression, except in lateral root primordia, indicating similarities between their promoter sequences (Chapter 2).

The $\mathrm{Zn}$ concentration in wild-type plants was neither different from the single mutants zip 3 and zip5 nor from the double mutant zip3/zip5 upon Zn deficiency supply. However, differences in $\mathrm{Zn}$ concentration were possible to be detected after Zn supply during a short period of time (Chapter 2). The double mutant was unable to uptake $\mathrm{Zn}$, as did the wild type and single mutants. This collection of results suggest that ZIP3 and ZIP5, have a redundant function in a subset of $\mathrm{Zn}$ homeostasis processes, but have independent functions in other subset of $\mathrm{Zn}$ homeostasis processes. The absence of a detectable phenotype, such as increased $\mathrm{Zn}$ sensitivity to $\mathrm{Zn}$ deficiency or decreased $\mathrm{Zn}$ concentration in single mutants of $\mathrm{Zn}$ transporters induced by $\mathrm{Zn}$ deficiency, is evidence of why alleles of these genes or single mutants have not been detected in forward genetic studies.

To better understand the function this set of $\mathrm{Zn}$ transporters induced by $\mathrm{Zn}$ deficiency, new combinations of double or triple mutants should be generated to identify groups of redundant genes. In addition, the phenotypes of $\mathrm{Zn}$ transporters mutants can be detected with targeted 
approaches, such as the use of fluorescein-based bright fluorescent Zn sensor Zinpyr-1 to detect Zn (Woodroofe et al., 2004). With the use of this fluorescent dye, I observed that in the root differentiation zone, most of the $\mathrm{Zn}$ concentrates in the stele, which could subsequently be translocated from root to shoot. Instead, in the Zn transporter mutants zip1, zip3, zip5 and ysl3, $\mathrm{Zn}$ is less concentrated in the stele. Thus, this targeted approach allows phenotypes in $\mathrm{Zn}$ transporters mutants to be detected (Chapter 2) (Sinclair et al., 2007). Another targeted approach could be the quantification of $\mathrm{Zn}$ concentration on specific cell layers by combining flow cytometry (Evrard et al., 2012; Galbraith, 2014) with Inductively Coupled Plasma Mass Spectrometry (ICP-MS) (Weis-Garcia et al., 2013). For this approach, the transgenic lines that I developed during my research thesis are useful. These lines express a nuclear localized Yellow Fluorescent Protein (NLS-SYFP2), of which, transcription is controlled by the promoters of $\mathrm{Zn}$ transporters. This lines could be used directly or after crosses with $\mathrm{Zn}$ transporter mutants. The cells in which these transporters are expressed can be isolated and their ionome analysed independently of the rest of the cellular population. Due to this fluorescent tags, we could identify that IRT3 expresses from the epidermis to stele, ZIPI gene expresses in endodermis and stele, ZIP3 and ZIP5 express in the epidermis and cortex, and ZIP11 and HMA2 express in xylem parenchyma cells (Chapter 2).

\section{Zn deficiency regulation at several levels}

Plants are sessile organisms that have developed different regulatory mechanisms to cope with acute stress. These mechanisms maximize their survival by rapid modifications of the physiology of the cells due to changes at the proteome level. The stress signalling networks responses can include targets regulating chromatin structure, transcription, post-transcription, translation and post-translation (Floris et al., 2009; Vert and Chory, 2009; de Nadal et al., 2011).

To search for $\mathrm{Zn}$ deficiency response, regulation components is a tempting option, considering the hidden nature of the phenotype of $\mathrm{Zn}$ transporters mutants. Up to this moment, the BASIC LEUCINE ZIPPER 19 (bZIP19) and bZIP23 are the only transcription factors known to regulate the $\mathrm{Zn}$ deficiency response in Arabidopsis (Assunção et al., 2010). However, the way how these two proteins sense $\mathrm{Zn}$ deficiency and respond to it to regulate the transcription of $\mathrm{Zn}$ transporters is not known (Assunção et al., 2013).

At the same time, that these two regulators were being discovered, the same group of researchers was running a project that identified the mutant studied in Chapter 3. The first goal 


\section{Discussion}

of the research described in Chapter 3 was to find regulators involved in the $\mathrm{Zn}$ deficiency response. The strategy consisted in using the promoter sequence of ZINC TRANSPORTER PROTEIN 4 (ZIP4) gene, a Zn deficiency highly induced gene (Chapter 2 and 3) (Wintz et al., 2003; Assunção et al., 2010) fused to the coding sequence of the B-GLUCURONIDASE (GUS) gene (Lin et al., 2016). The $\mathrm{Zn}$ deficiency screenable line created in this way, was subject to random mutation. Mutants were screened for a $\mathrm{Zn}$ deficiency response suppressed or constantly activated in the plant. The mutant line obtained in this research showed the $\mathrm{Zn}$ deficiency response upon $\mathrm{Zn}$ deficiency and $\mathrm{Zn}$ sufficiency. The mutation responsible for this effect is localized in the N-ALPHA-TERMINAL-ACETYLTRANSFERASE 25 (NAA25) gene, which encodes for the non-catalytic subunit of $\mathrm{N}$-alpha-terminal-acetyltransferase complex B (NatB). This complex carries out the N-t-acetylation of proteins during translation (Polevoda and Sherman, 2000; Gibbs, 2015; Aksnes et al., 2016).

The gene found in the mutant screening does not encode for a transcription regulator as originally expected. Instead, the NatB complex is responsible for the acetylation of around $15 \%$ of the Arabidopsis proteome (Linster et al., 2015; Aksnes et al., 2016). This result significantly complicated the discovery of a $\mathrm{Zn}$ deficiency response regulator, because of the large number of NatB targets in the proteome prone to modification. Protein modifications can affect stability (Schrader et al., 2009), subcellular location, interaction with membranes, protein-protein interactions, protein folding and aggregation (Friso and van Wijk, 2015).

Among the putative targets of NatB are regulators of the $\mathrm{Zn}$ deficiency response, such as bZIP19 and bZIP23. Assunção et al. (2010, 2013) hypothesized that these proteins can modify their function by sensing $\mathrm{Zn}$ in the medium, because their expression is not significantly increased due to $\mathrm{Zn}$ deficiency. If indeed, these two proteins are acetylated by the NatB, the disturbed acetylation in the naa25 mutant lines, could affect their function causing the expression of $\mathrm{Zn}$ transporters even upon $\mathrm{Zn}$ sufficiency. $\mathrm{Zn}$ transporters induced by $\mathrm{Zn}$ deficiency show a slightly but consistently high expression in the naa25 mutant upon $\mathrm{Zn}$ sufficiency compared to wild type. In addition, the naa25 mutant lines were highly sensitive to Fe deficiency. The Fe deficiency cascade regulators, BASIC HELIX-LOOP-HELIXE 104 (bHLH104) and FER-LIKE IRON DEFICIENCY-INDUCED TRANSCRIPTION FACTOR (FIT) (Yuan et al., 2008; Zhang et al., 2015; Li et al., 2016) are putative targets of NatB as well. Thus, the effects of the naa25 loss of function in the $\mathrm{Zn}$ and Fe homeostasis can be as a result of the lack of acetylation of their regulators or by pleiotropic effects for the disturbance of other 
processes in the plant. The plant immunity is disturbed by mutations in the genes encoding the catalytic and auxiliary subunit of NatB, which decreases the stability of the plant immune receptor SUPPRESSOR OF NPR1 CONSTITUTIVE 1 (SNC1) (Xu et al., 2015).

Little is known about how co- and post-translational modifications affect the regulation of elements homeostasis in plants. Several element transporters are target co- and posttranslational modifications that affect their stability and specificity. High concentration of Boron (B) induces ubiquitination of the BORON TRANSPORTER 1 (BOR1), this ubiquitination is crucial for the protein sorting and degradation in the vacuole (Kasai et al., 2011). The heavy metal transporter receptor IRON-REGULATED TRANSPORTER 1 (IRT1) is degraded by phosphorylation to avoid the heavy metal accumulation of unspecific substrates such as $\mathrm{Zn}$ to ensure proper Fe uptake (Dubeaux et al., 2018). The phosphorylation of the HIGH-AFFINITY K TRANSPORTER 5 (HAK5), K TRANSPORTER 1 (AKT1), and the nitrate transporter NITRATE TRANSPORTER 1 (NTR1) increases the affinity to their respective substrates, upon low supply, while the de-phosphorylated state has a lower affinity (Xu et al., 2006; Ho et al., 2009; Ragel et al., 2015). In the case of $\mathrm{Zn}$ homeostasis, there is still a long way to go learning about co- and post-translational modifications that suffer the $\mathrm{Zn}$ homeostasis players at the protein level. The aberrant $\mathrm{Zn}$ deficiency response in the naa25 mutant is a hint to test the in vivo bZIP19 and bZIP23 N-t-acetylation, and its possible effects over their regulatory function.

\section{Natural variation to uncover tolerance to $\mathrm{Zn}$ deficiency}

Mining the natural variation of a species is a way to discover new allele variants that are useful to uncover the function of genes. For that reason, I explored the ionome natural variation of Arabidopsis in search of genes involved in the $\mathrm{Zn}$ deficiency tolerance. Arabidopsis natural accessions have a high level of variation for several traits (Koornneef and Meinke, 2010) including the responses to $\mathrm{Zn}$ deficiency (Campos et al., 2017). The analyses of the variation of specific traits can be done in several ways, for instance by constructing biparental populations with extreme phenotypes or by using large populations in Genome-Wide Association studies (GWAS) (Nordborg and Weigel, 2008).

I followed the GWAS approach inspired in the successful examples where allelic variation was detected for the HIGH-AFFINITY K TRANSPORTER 1 (HKT1) gene (Baxter et al., 2010), for the arsenate reductase HIGH ARSENIC CONTENT 1 (HAC1) gene (Chao et al., 2014), and the 


\section{Discussion}

Cd transporter HEAVY-METAL ATPASE 3 (HMA3) gene (Chao et al., 2012). At this point, we need to keep in mind that the homeostasis of essential nutrients requires a tight control of the symplast concentration of each nutrient to meet the physiological requirements and avoid toxicity (Hindt and Guerinot, 2012; Sinclair and Krämer, 2012; Hermans et al., 2013; Printz et al., 2016). Therefore, in the homeostasis of essential nutrients a large number of players will be involved (Hindt and Guerinot, 2012; Sinclair and Krämer, 2012; Hermans et al., 2013; Bashir et al., 2016; Printz et al., 2016) with redundant functions (Chapter 2). Homeostasis of essential nutrients likely requires functional alleles and often does not favour loss-of-function alleles, as were discovered for the $H M A 3$ and $H A C 1$ genes. In addition, the tolerance to non-essential elements requires the exclusion of the element from the cells symplast, which likely requires a lower number of genes than the ones involved in essential nutrients homeostasis.

The best results obtained by GWAS are in traits driven by a small number of loci with large effect sizes (Atwell et al., 2010). This implies several current problems in working with complex traits. As mentioned in the infinitesimal model of inheritance of Fisher (Fisher, 1919), traits are driven by many loci with small effects on each one. Large-effect variants are low frequent (Rockman, 2012; Marjoram et al., 2014) and the effect of specific SNPs needs to be sufficiently frequent in the population to be easily identified (Korte and Farlow, 2013). In addition, causal variants can be poorly tagged with makers, the reason why they will not be detected (Gusev et al., 2013). Due to the polygenic nature of $\mathrm{Zn}$ homeostasis (Sinclair and Krämer, 2012) and presence of $\mathrm{Zn}$ transporters being functionally redundant (Chapter 2), a low success was expected in finding associated SNPs in genes encoding for Zn transporters when $\mathrm{Zn}$ concentration was the trait analysed.

Plants suffering from $\mathrm{Zn}$ deficiency show a significant decrease in $\mathrm{Zn}$ concentration and a generalized disequilibrium of the plant ionome (Chapter 4) (Campos et al., 2017). Thus, in addition to $\mathrm{Zn}$ concentration, I also used in the GWAS the concentrations of $\mathrm{Co}, \mathrm{Cu}, \mathrm{Mn}, \mathrm{Mo}$, and $\mathrm{Fe}$, which are micronutrients related to $\mathrm{Zn}$. Associations were detected between $\mathrm{Mn}$ concentrations and SNPs in the Mn transporter NATURAL RESISTANCE-ASSOCIATED MACROPHAGE PROTEIN 1 (NRAMP1) gene (Cailliatte et al., 2010; Castaings et al., 2016) and between Mo concentrations and SNPs in the MOLYBDATE TRANSPORTER 1 (MOT1) (Tomatsu et al., 2007; Baxter et al., 2008). Apart from these two genes, there was not any other micronutrient homeostasis player associated with the element its homeostasis is involved in. Thus, most of the candidate genes selected in Chapter 4 do not have a known function related 
with micronutrients homeostasis, but their mutants showed significant changes in element concentrations.

The analyses of the candidate genes mutants ton 1 recruiting motif 22 (trm22), at5g66890, pigment defective 318 (pde318), target of early activation tagged (toe3), early flowering 3 (elf3), at lg49350, and ferritin 4 (fer4) showed a significant difference with the wild type, in the concentration of the element, each gene was associated with in the GWAS. Zinc deficient plants show a decrease in the $\mathrm{Zn}$ concentration and significant changes in the concentration in most of the elements of the ionome (Ghandilyan et al., 2012; Campos et al., 2017). Therefore, the tolerance to $\mathrm{Zn}$ deficiency could be reflected in the lesser changes in the ionome due to $\mathrm{Zn}$ deficiency. The changes in the element concentrations due to $\mathrm{Zn}$ deficiency intensify in the mutants trm22, at5g66890, pde318, toe3, elf3, at 1 49350, and fer4 when compared to wild type. Some of the elements related to $\mathrm{Zn}$ with significant changes in these mutants are Fe, Mn, and $\mathrm{Cu}$. And these elements are required for essential plant process like photosynthesis (Varotto et al., 2002; Kessler and Papenbrock, 2005; Yruela, 2013). The changes in concentration in non-targeted elements, as observed in the mutants studied, could be either a biological pleiotropy or a mediated pleiotropy. In a biological pleiotropy, the gene has a direct biological influence on more than one trait. In a mediated pleiotropy, one phenotype is itself causally related to a second phenotype (Solovieff et al., 2013). Therefore, by using the ionome of plants grown upon Zn sufficiency or Zn deficiency, I could identify genes, in which loss of function increases the ionome disequilibrium in the plant due to $\mathrm{Zn}$ deficiency. Probably, these genes were associated with micronutrients concentration due to their effect in several traits, which caused major disequilibrium in the ionome of the plant (Chapter 4).

To identify genetic variants of genes directly involved in $\mathrm{Zn}$ homeostasis, more specific questions, and targeted approaches should be applied. A good example of a specific question and targeted approach in applying GWAS was performed for developmental cell-type traits, in which the length of the root meristematic zone was associated with F-box gene $K U R Z U N D$ $K L E I N(K U K)$ that was confirmed to regulate meristem length (Meijón et al., 2014). Thus, in $\mathrm{Zn}$ homeostasis, we should try to isolate the process, as much as possible to be able to disentangle their players one-by-one. In this way, we can avoid the effects of genes controlling other processes. For instance, if we are looking to find $\mathrm{Zn}$ homeostasis players in roots, probably individual cell layers should be analysed for Zn concentration. In this case, cell sorting by flow cytometry plus ICP-MS (Weis-Garcia et al., 2013), laser micro-dissection, plus ICP-MS or laser 


\section{Discussion}

ablation-inductively coupled plasma-mass spectrometry (Persson et al., 2016) could be applied. The analysing of the $\mathrm{Zn}$ distribution in the root cellular layers with $\mathrm{Zn}$ dyes as Zinpyr-1 can be useful because individual $\mathrm{Zn}$ transporter mutants can be spotted in this way (Chapter 2) (Sinclair et al., 2007). Also, the analyses of $\mathrm{Zn}$ uptake in short periods of time as shown in Chapter 2 could be suitable to detect basal uptake mechanisms.

\section{Tolerance to abiotic stress}

Plants either in natural or agricultural environments occupy a wide range of ecological niches, to which they are exposed to biotic and abiotic stresses. The defence or tolerance response to stress requires the reallocation of resources to cope with the environmental challenge. The activation of responses to stress often leads to an overall reduced plant growth, for instance, mutant plants with enhanced autoimmune response are small. Thus, plants need to effectively fine-tune the trade-offs between response to the stress and the developmental process in a costefficient manner to deal with stress and coordinate growth (Zhang et al., 2003; Vinocur and Altman, 2005; Lozano-Durán and Zipfel, 2015; Haina and Uwe, 2017).

Growth hormone networks are regulated during stress responses to coordinate growth (Haina and Uwe, 2017). Brassinosteroids (BRs) are positive growth regulators that merge signals from biotic stress with growth and developmental plant programs. Thus, BRs are regulators of tradeoffs between disease resistance and plant growth (De Bruyne et al., 2014). BRs also link abiotic signals to growth. During drought or carbon starvation, the BRI 1-EMS-SUPPRESSOR 1 (BES1), a transcription factor part of the BRs signalling, is degraded affecting the normal plant growth rate. This mechanism favours the plant survival under these stress conditions at the cost of plant growth (Nolan et al., 2017). Gibberellic acid is also a positive growth phytohormone; its signalling is repressed by DELLA proteins (DELLAs). DELLAs are transcription factors, with growth repressing effects enhanced by stress conditions as salt (Silverstone et al., 1997; Achard et al., 2006; Sun, 2010).

The abiotic stress caused by shortage of nutrients such as $\mathrm{Zn}$ is generally characterized by chlorosis and stunted growth among other symptoms, which increase with the severity of the stress and time of exposure (Cakmak, 2008; Alloway, 2009; Marschner and Marschner, 2012; Yruela, 2013; Campos, 2015; Mattiello et al., 2015). Tolerance to $\mathrm{Zn}$ deficiency stress is variable among Arabidopsis natural accessions (Campos et al., 2017), which could be expressed in terms of ionome equilibrium, sufficient $\mathrm{Zn}$ concentration (Chapter 4) or less severity of the 
Zn deficiency symptoms in the plant (Hacisalihoglu and Kochian, 2003; Cakmak, 2008; Alloway, 2009; Campos et al., 2017). In Chapter 4, after applying a GWAS, I found an overrepresentation of HEAVY METAL-ASSOCIATED ISOPRENYLATED PLANT PROTEINS $(H I P P)$ genes harbouring SNPs associated with the ionome of Arabidopsis natural accessions grew upon sufficient or deficient Zn supply.

HIPPS are involved in biotic and especially in abiotic stress responses. Cold affects the expression of HIPP24, HIPP26, HIPP23, HIPP25 and HIPP27 (Barth et al., 2009), drought and abscisic acid affect the expression of HIPP3 (Zschiesche et al., 2015). HIPPs role has been mainly related to tolerance to toxic elements like $\mathrm{Cd}$. The expression of HIPP26 is induced by cold, drought, salt, and Cd (Barth et al., 2009; Gao et al., 2009), and its overexpression induces tolerance to $\mathrm{Cd}$ (Gao et al., 2009). In a similar way, HIPP6 overexpression confers tolerance to $\mathrm{Cd}$ and its expression is induced by Cd, Mercury (Hg), Fe, and Cu (Suzuki et al., 2002). The triple mutant loss of function hipp20/hipp21/hipp22 is highly sensitive to Cd compared to wildtype plants (Tehseen et al., 2010). As a common pattern, stress conditions change the HIPPs gene expression and are involved in the tolerance to the non-essential heavy metal $\mathrm{Cd}$.

I performed functional analyses in five HIPPs not described at the moment, which were detected in the GWAS of Chapter 4, and are part of an intriguing cluster of tandemly arrayed genes. Mutants of these genes showed increased tolerance to $\mathrm{Zn}$ deficiency (Chapter 5). However, after testing several stress conditions, I found out that their function is not specific for $\mathrm{Zn}$ deficiency. Mutants of this cluster of tandemly arrayed HIPPs also showed increase tolerance to $\mathrm{Zn}$ excess, Fe excess, and especially to Fe deficiency. The expression of these genes is induced upon Zn deficiency or Fe deficiency in wild-type plants. The overexpression lines of one of these genes showed to be as sensitive as the wild type, and in some cases even more sensitive than wild type to deficient or excess Fe or Zn supply. In addition, members of this cluster are higher expressed in the acetylation mutant naa 25 when compared to wild type. The naa25 mutant is more sensitive to Fe deficiency than the wild type (Chapter 3) and has a disturbed $\mathrm{Zn}$ deficiency response. These results suggest the role of the five members of the cluster of tandemly arrayed HIPPs in adverse $\mathrm{Zn}$ and Fe supply.

The five genes from this cluster had not been characterized to the moment, due to sequence similarities they are related to metallochaperons (Tehseen et al., 2010; de Abreu-Neto et al., 2013). The heavy metal domain of these HIPPs contains the general sequence of a heavy metal 


\section{Discussion}

binding domain but lacks its core motif (Tehseen et al., 2010), the reason why this could be a heavy metal-associated like domain (Chapter 5). HIPPs like HIPP3, HIPP7 and HIPP26 have been experimentally shown to bind heavy metals (Dykema et al., 1999; Achard et al., 2006; Gao et al., 2009). However, it is not known if this domain is functional or not in the five members of the cluster of tandemly arrayed HIPPs. If it is functional, it could be involved in buffering metals in the symplast, trafficking metals in the cell (Wintz and Vulpe, 2002; Robinson and Winge, 2010; Pang et al., 2013) or sensing metals concentrations, which is the case of metalloregulatory proteins (Giedroc and Arunkumar, 2007; Kim et al., 2007; Lee and Helmann, 2007; Waldron et al., 2009; Caballero et al., 2011).

Functional analyses of the cluster of tandemly array HIPPs suggest a role in gene transcription regulation. Members of this cluster, positively regulates the expression of the other members of the same cluster upon Fe deficiency, either by direct or indirect interactions. When genes participate in similar pathways, their cross-regulation can become a necessary mechanism to ensure that all the components of a process be present at the same moment, especially if they interact among them (Gómez-Mena et al., 2005; Immink et al., 2009). The interaction among members of the cluster of tandemly array HIPPs is a feasible hypothesis, because these HIPPs have some common interactors among them, suggesting their work as a complex of proteins. HIPPs interact with several nuclear-localized proteins. And HIPPs interaction partners show interactions among them as well, which indicates that these HIPPs belong to a highly connected network of proteins.

The processes, in which these HIPPs are involved can be inferred by the function of their interaction partners. The proteins with which HIPPs interact are: NO APICAL MERISTEM/ARABIDOPSIS TRANSCRIPTION ACTIVATION FACTOR/CUP-SHAPED COTYLEDON DOMAIN CONTAINING PROTEIN 001 (NAC001), NAC13, NAC016, NAC069, NAC089, MEMBRANE-BOUND NAC 8 (NTL8), NTL9, bHLH112, ZINC FINGER HOMEOBOX PROTEIN 4 (ZFHD4), ETHYLENE RESPONSE FACTOR 120 (ERF120), HYPERSENSITIVITY TO LOW PI-ELICITED PRIMARY ROOT SHORTENING 1 HOMOLOGUE 1 (HHO1), REGULATORS OF AXILLARY MERISTEMS 3 (RAX3), TRF5, ASIL1, DOF2, BEL1, GOLDEN2-LIKE 1 (GLK1), GLK2, F15L12, AT3G54390, ETHYLENE INSENSTIVE 2 NUCLEAR-ASSOCIATED PROTEIN 1 (ENAP1) and ENAP2. The HIPPs interaction partners have regulatory functions and several of them link stress signals to plant growth and development. The stress signals they link come 
from high or low nutrients supply (Tan and Irish, 2006; Kasajima and Fujiwara, 2007; Cheng et al., 2012; Medici et al., 2015; Pan et al., 2015; Liu et al., 2017), salinity (Kim et al., 2008; Kim et al., 2013; He et al., 2015; Liu et al., 2015; Sakuraba et al., 2016), and drought (Liu et al., 2015). And those signals affect processes as germination (Kim et al., 2008; Rueda-Romero et al., 2012; He et al., 2015), growth (Kim et al., 2007; Cheng et al., 2012; Pan et al., 2015; Zhang et al., 2016; Liu et al., 2017; Zhang et al., 2017; Zhang et al., 2018), meristem initiation (Müller et al., 2006), senescence (Sakuraba et al., 2016), and cell death (Klein et al., 2012; De Clercq et al., 2013). Notably, members of the cluster of tandemly arrayed HIPPs and some of their interactors show a similar pattern, which consisted in gene expression induced by stress, loss of function of the genes increases tolerance to stress, and the overexpression of genes decreased growth. Which is similar to the negative-plant-growth regulators DELLAs (Achard et al., 2006).

All the results obtained from the cluster of tandemly arrayed HIPPs suggest their role as negative-plant-growth regulators induced by stress. The HIPP genes came to my hands in a study looking for genes involved in tolerance to $\mathrm{Zn}$ deficiency. Indeed, these genes show to be involved in Zn deficiency tolerance. However, they are not specific for Zn deficiency, their loss of function provide tolerance to adverse $\mathrm{Zn}$ and Fe supply, and maybe to more stress conditions than the ones tested in this research. Still, many aspects of the function of these HIPPs need to be studied, such as the genes whose expressions they may affect, trade-offs of their loss of function, and signal molecules in their regulatory activity. These genes have the potential to be used later on to improve crop tolerance and the information obtained in this thesis can be only the tip of the iceberg their potential.

\section{Final remarks}

$\mathrm{Zn}$ deficiency response is a complex trait constituted by several components, among them $\mathrm{Zn}$ transporters, mainly in charged to increase the $\mathrm{Zn}$ symplast concentration. The expression of $\mathrm{Zn}$ transporters is induced only six hours after exposure to $\mathrm{Zn}$ deficiency indicating the existence of a mechanism in charge to sense the shortage of $\mathrm{Zn}$ and regulate gene expression. The transcription factors bZIP19 and bZIP23 induce the expression of several Zn transporters during Zn deficiency. Furthermore, we do not know for sure about other levels of regulation of the $\mathrm{Zn}$ homeostasis. Probably, the acetylation of bZIP19 and bZIP23 is a required step to allow the proper function of these transcription factors. The phenotypes of $\mathrm{Zn}$ transporters induced by deficiency are difficult to detect, mainly due to functional redundancy among some of them. 
This characteristic complicates the detection of $\mathrm{Zn}$ transporter mutants in forward genetic approaches. The genes, in which mutation causes significant changes in several elements of the plant ionome upon $\mathrm{Zn}$ deficiency, were identified by forward genetics using the ionome natural variation, as well as genes, in which mutation significantly improved the tolerance to $\mathrm{Zn}$ deficiency, $\mathrm{Zn}$ excess, Fe deficiency and Fe excess were identified by forward genetics using the ionome natural variation.

A deep understanding of the genetic architecture of $\mathrm{Zn}$ deficiency is a key step in improving the crops efficient use of nutrients and increasing the $\mathrm{Zn}$ content of the edible parts of crops, through plant breeding strategies. 


References 
Achard P, Cheng H, De Grauwe L, Decat J, Schoutteten H, Moritz T, Van Der Straeten D, Peng J, Harberd NP (2006) Integration of Plant Responses to Environmentally Activated Phytohormonal Signals. Science 311: 91-94

Achard P, Genschik P (2009) Releasing the brakes of plant growth: how GAs shutdown DELLA proteins. Journal of Experimental Botany 60: 1085-1092

Achard P, Renou J-P, Berthomé R, Harberd NP, Genschik P (2008) Plant DELLAs Restrain Growth and Promote Survival of Adversity by Reducing the Levels of Reactive Oxygen Species. Current Biology 18: 656-660

Ackland ML, Michalczyk AA (2016) Zinc and infant nutrition. Archives of Biochemistry and Biophysics 611: 51-57

Ahn SJ, Shin R, Schachtman DP (2004) Expression of KT/KUP Genes in Arabidopsis and the Role of Root Hairs in K+ Uptake. Plant Physiology 134: 1135-1145

Aksnes H, Drazic A, Marie M, Arnesen T (2016) First Things First: Vital Protein Marks by N-Terminal Acetyltransferases. Trends in Biochemical Sciences 41: 746-760

Al-Shehbaz IA, O'Kane SL (2002) Taxonomy and Phylogeny of Arabidopsis (Brassicaceae). The Arabidopsis Book / American Society of Plant Biologists 1: e0001

Allen RS, Li J, Stahle MI, Dubroué A, Gubler F, Millar AA (2007) Genetic analysis reveals functional redundancy and the major target genes of the Arabidopsis miR159 family. Proceedings of the National Academy of Sciences 104: 16371-16376

Alloway BJ (2009) Soil factors associated with zinc deficiency in crops and humans. Environmental Geochemistry and Health 31: 537-548

Alonso-Blanco C, Aarts MGM, Bentsink L, Keurentjes JJB, Reymond M, Vreugdenhil D, Koornneef M (2009) What has natural variation taught us about plant development, physiology, and adaptation? Plant Cell 21: 1877-1896

Anderegg G, Ripperger H (1989) Correlation between metal complex formation and biological activity of nicotianamine analogues. Journal of the Chemical Society, Chemical Communications: 647-650

Anders S, Pyl PT, Huber W (2015) HTSeq - a Python framework to work with highthroughput sequencing data. Bioinformatics 31: 166-169

Andreini C, Banci L, Bertini I, Rosato A (2006) Counting the Zinc-Proteins Encoded in the Human Genome. Journal of Proteome Research 5: 196-201

Andrés-Colás N, Perea-García A, Puig S, Peñarrubia L (2010) Deregulated Copper Transport Affects Arabidopsis Development Especially in the Absence of Environmental Cycles. Plant Physiology 153: 170-184 
Araki R, Shikanai T (2014) Analysis of photosynthetic electron transport in Arabidopsis thaliana under iron deficiency. Journal of Crop Research 59: 11-15

Arosio P, Ingrassia R, Cavadini P (2009) Ferritins: A family of molecules for iron storage, antioxidation and more. Biochimica et Biophysica Acta (BBA) - General Subjects 1790: 589-599

Arrivault S, Senger T, Krämer U (2006) The Arabidopsis metal tolerance protein AtMTP3 maintains metal homeostasis by mediating $\mathrm{Zn}$ exclusion from the shoot under $\mathrm{Fe}$ deficiency and Zn oversupply. Plant Journal 46: 861-879

Assunção AGL, Herrero E, Lin YF, Huettel B, Talukdar S, Smaczniak C, Immink RGH, Van Eldik M, Fiers M, Schat H, Aarts MGM (2010) Arabidopsis thaliana transcription factors bZIP19 and bZIP23 regulate the adaptation to zinc deficiency. Proceedings of the National Academy of Sciences of the United States of America 107: 10296-10301

Assunção AGL, Persson DP, Husted S, Schjørring JK, Alexander RD, Aarts MGM (2013) Model of how plants sense zinc deficiency. Metallomics 5: 1110-1116

Assunção AGL, Schat H, Aarts MGM (2003) Thlaspi caerulescens, an attractive model species to study heavy metal hyperaccumulation in plants. New Phytologist 159: 351360

Atwell S, Huang YS, Vilhjalmsson BJ, Willems G, Horton M, Li Y, Meng D, Platt A, Tarone AM, Hu TT, Jiang R, Muliyati NW, Zhang X, Amer MA, Baxter I, Brachi B, Chory J, Dean C, Debieu M, de Meaux J, Ecker JR, Faure N, Kniskern JM, Jones JDG, Michael T, Nemri A, Roux F, Salt DE, Tang C, Todesco M, Traw MB, Weigel D, Marjoram P, Borevitz JO, Bergelson J, Nordborg M (2010) Genomewide association study of 107 phenotypes in Arabidopsis thaliana inbred lines. 465: 627-631

Avice J-C, Etienne P (2014) Leaf senescence and nitrogen remobilization efficiency in oilseed rape (Brassica napus L.). Journal of Experimental Botany 65: 3813-3824

Azimzadeh J, Nacry P, Christodoulidou A, Drevensek S, Camilleri C, Amiour N, Parcy F, Pastuglia M, Bouchez D (2008) Arabidopsis TONNEAU1 Proteins Are Essential for Preprophase Band Formation and Interact with Centrin. The Plant Cell 20: 21462159

Aznar A, Chen NWG, Rigault M, Riache N, Joseph D, Desmaële D, Mouille G, Boutet S, Soubigou-Taconnat L, Renou J-P, Thomine S, Expert D, Dellagi A (2014) 
Scavenging Iron: A Novel Mechanism of Plant Immunity Activation by Microbial Siderophores. Plant Physiology 164: 2167-2183

Aznar A, Chen NWG, Thomine S, Dellagi A (2015) Immunity to plant pathogens and iron homeostasis. Plant Science 240: 90-97

Barak P, Helmke PA (1993) The Chemistry of Zinc. In AD Robson, ed, Zinc in Soils and Plants: Proceedings of the International Sym posium on 'Zinc in Soils and Plants' held at The University of Western Australia, 27-28 September, 1993. Springer Netherlands, Dordrecht, pp 1-13

Barberon M, Geldner N (2014) Radial Transport of Nutrients: The Plant Root as a Polarized Epithelium. Plant Physiology 166: 528-537

Barth O, Vogt S, Uhlemann R, Zschiesche W, Humbeck K (2009) Stress induced and nuclear localized HIPP26 from Arabidopsis thaliana interacts via its heavy metal associated domain with the drought stress related zinc finger transcription factor ATHB29. Plant Molecular Biology 69: 213-226

Bashir K, Rasheed S, Kobayashi T, Seki M, Nishizawa NK (2016) Regulating Subcellular Metal Homeostasis: The Key to Crop Improvement. Frontiers in Plant Science 7: 1192

Baxter I, Brazelton JN, Yu D, Huang YS, Lahner B, Yakubova E, Li Y, Bergelson J, Borevitz JO, Nordborg M, Vitek O, Salt DE (2010) A Coastal Cline in Sodium Accumulation in Arabidopsis thaliana Is Driven by Natural Variation of the Sodium Transporter AtHKT1;1. PLoS Genet 6: e1001193

Baxter I, Hermans C, Lahner B, Yakubova E, Tikhonova M, Verbruggen N, Chao D-y, Salt DE (2012) Biodiversity of Mineral Nutrient and Trace Element Accumulation in Arabidopsis thaliana. PLoS ONE 7: e35121

Baxter I, Muthukumar B, Park HC, Buchner P, Lahner B, Danku J, Zhao K, Lee J, Hawkesford MJ, Guerinot ML, Salt DE (2008) Variation in Molybdenum Content Across Broadly Distributed Populations of Arabidopsis thaliana Is Controlled by a Mitochondrial Molybdenum Transporter (MOT1). PLOS Genetics 4: e1000004

Baxter IR, Vitek O, Lahner B, Muthukumar B, Borghi M, Morrissey J, Guerinot ML, Salt DE (2008) The leaf ionome as a multivariable system to detect a plant's physiological status. Proceedings of the National Academy of Sciences of the United States of America 105: 12081-12086

Beal T, Massiot E, Arsenault JE, Smith MR, Hijmans RJ (2017) Global trends in dietary micronutrient supplies and estimated prevalence of inadequate intakes. PLOS ONE 12: $\mathrm{e} 0175554$ 
Bellaoui M, Pidkowich MS, Samach A, Kushalappa K, Kohalmi SE, Modrusan Z, Crosby WL, Haughn GW (2001) The Arabidopsis BELL1 and KNOX TALE Homeodomain Proteins Interact through a Domain Conserved between Plants and Animals. The Plant Cell 13: 2455-2470

Beneš I, Schreiber K, Ripperger H, K ircheiss A(1983) Metal complex formation by nicotianamine, a possible phytosiderophore. Experientia 39: 261-262

Benjamini Y, Hochberg Y (1995) Controlling the False Discovery Rate: A Practical and Powerful Approach to Multiple Testing. Journal of the Royal Statistical Society. Series B (Methodological) 57: 289-300

Bird AJ, McCall K, Kramer M, Blankman E, Winge DR, Eide DJ (2003) Zinc fingers can act as $\mathrm{Zn} 2+$ sensors to regulate transcriptional activation domain function. The EMBO journal 22: 5137-5146

Biswas KK, Ooura C, Higuchi K, Miyazaki Y, Van Nguyen V, Rahman A, Uchimiya H, Kiyosue T, Koshiba T, Tanaka A, Narumi I, Oono Y (2007) Genetic Characterization of Mutants Resistant to the Antiauxin p-Chlorophenoxyisobutyric Acid Reveals That AAR3, a Gene Encoding a DCN1-Like Protein, Regulates Responses to the Synthetic Auxin 2,4-Dichlorophenoxyacetic Acid in Arabidopsis Roots. Plant Physiology 145: $773-785$

Black RE, Allen LH, Bhutta ZA, Caulfield LE, de Onis M, Ezzati M, Mathers C, Rivera J (2008) Maternal and child undernutrition: global and regional exposures and health consequences. The Lancet 371: 243-260

Blindauer CA, Schmid R (2010) Cytosolic metal handling in plants: Determinants for zinc specificity in metal transporters and metallothioneins. Metallomics 2: 510-529

Bonferroni CE (1935) Il calcolo delle assicurazioni su gruppi di teste. Tipografia del Senato

Bradshaw HD (2005) Mutations in CAX1 produce phenotypes characteristic of plants tolerant to serpentine soils. New Phytologist 167: 81-88

Briat J-F, Rouached H, Tissot N, Gaymard F, Dubos C (2015) Integration of P, S, Fe, and Zn nutrition signals in Arabidopsis thaliana: potential involvement of PHOSPHATE STARVATION RESPONSE 1 (PHR1). Frontiers in Plant Science 6

Buescher E, Achberger T, Amusan I, Giannini A, Ochsenfeld C, Rus A, Lahner B, Hoekenga O, Yakubova E, Harper JF, Guerinot ML, Zhang M, Salt DE, Baxter IR (2010) Natural Genetic Variation in Selected Populations of Arabidopsis thaliana is Associated with Ionomic Differences. PLoS ONE 5: e11081 
Buljan M, Bateman A (2009) The evolution of protein domain families. Biochemical Society Transactions 37: 751-755

Caballero HR, Campanello GC, Giedroc DP (2011) Metalloregulatory Proteins: Metal Selectivity and Allosteric Switching. Biophysical chemistry 156: 103-114

Cailliatte R, Schikora A, Briat J-F, Mari S, Curie C (2010) High-Affinity Manganese Uptake by the Metal Transporter NRAMP1 Is Essential for Arabidopsis Growth in Low Manganese Conditions. The Plant Cell 22: 904-917

Cakmak I (2008) Enrichment of cereal grains with zinc: Agronomic or genetic biofortification? Plant and Soil 302: 1-17

Campos ACAL (2015) Study of natural variation for $\mathrm{Zn}$ deficiency tolerance in Arabidopsis thaliana. Wageningen University, Wageningen

Campos ACAL, Kruijer W, Alexander R, Akkers RC, Danku J, Salt DE, Aarts MGM (2017) Natural variation in Arabidopsis thaliana reveals shoot ionome, biomass, and gene expression changes as biomarkers for zinc deficiency tolerance. Journal of Experimental Botany 68: 3643-3656

Castaings L, Caquot A, Loubet S, Curie C (2016) The high-affinity metal Transporters NRAMP1 and IRT1 Team up to Take up Iron under Sufficient Metal Provision. 6: 37222

Caulfield LE, Black RE (2004) Zinc deficiency. Comparative Quantification of Health Risks: Global and Regional Burden of Disease Attributable to Selected Major Risk Factors: 257-279

Chao D-Y, Baraniecka P, Danku J, Koprivova A, Lahner B, Luo H, Yakubova E, Dilkes B, Kopriva S, Salt DE (2014) Variation in Sulfur and Selenium Accumulation Is Controlled by Naturally Occurring Isoforms of the Key Sulfur Assimilation Enzyme ADENOSINE 5'-PHOSPHOSULFATE REDUCTASE2 across the Arabidopsis Species Range. Plant Physiology 166: 1593-1608

Chao D-Y, Chen Y, Chen J, Shi S, Chen Z, Wang C, Danku JM, Zhao F-J, Salt DE (2014) Genome-wide Association Mapping Identifies a New Arsenate Reductase Enzyme Critical for Limiting Arsenic Accumulation in Plants. PLoS Biol 12: e1002009

Chao D-Y, Silva A, Baxter I, Huang YS, Nordborg M, Danku J, Lahner B, Yakubova E, Salt DE (2012) Genome-Wide Association Studies Identify Heavy Metal ATPase3 as the Primary Determinant of Natural Variation in Leaf Cadmium in Arabidopsis thaliana. PLoS Genet 8: e1002923 
Chen X, Ludewig U (2018) Biomass increase under zinc deficiency caused by delay of early flowering in Arabidopsis. Journal of Experimental Botany 69: 1269-1279

Chen Y-Y, Wang Y, Shin L-J, Wu J-F, Shanmugam V, Tsednee M, Lo J-C, Chen C-C, Wu S-H, Yeh K-C (2013) Iron Is Involved in the Maintenance of Circadian Period Length in Arabidopsis. Plant Physiology 161: 1409-1420

Cheng M-C, Hsieh E-J, Chen J-H, Chen H-Y, Lin T-P (2012) Arabidopsis RGLG2, Functioning as a RING E3 Ligase, Interacts with AtERF53 and Negatively Regulates the Plant Drought Stress Response. Plant Physiology 158: 363-375

Cheung M-Y, Li X, Miao R, Fong Y-H, Li K-P, Yung Y-L, Yu M-H, Wong K-B, Chen Z, Lam H-M (2016) ATP binding by the P-loop NTPase OsYchF1 (an unconventional G protein) contributes to biotic but not abiotic stress responses. Proceedings of the National Academy of Sciences 113: 2648-2653

Choi J, Choi D, Lee S, Ryu C-M, Hwang I Cytokinins and plant immunity: old foes or new friends? Trends in Plant Science 16: 388-394

Clarkson DT (1993) Roots and the Delivery of Solutes to the Xylem. Philosophical Transactions of the Royal Society of London. Series B: Biological Sciences 341: 5-17

Claus J, Bohmann A, Chavarría-Krauser A (2013) Zinc uptake and radial transport in roots of Arabidopsis thaliana: A modelling approach to understand accumulation. Annals of Botany 112: 369-380

Clough SJ, Bent AF (1998) Floral dip: A simplified method for Agrobacterium-mediated transformation of Arabidopsis thaliana. Plant Journal 16: 735-743

Clouse SD (2011) Brassinosteroid Signal Transduction: From Receptor Kinase Activation to Transcriptional Networks Regulating Plant Development. The Plant Cell 23: 1219-1230

Colangelo EP, Guerinot ML (2004) The Essential Basic Helix-Loop-Helix Protein FIT1 Is Required for the Iron Deficiency Response. The Plant Cell 16: 3400-3412

Connolly EL, Guerinot ML (2002) Iron stress in plants. Genome Biology 3: reviews1024.1021-reviews1024.1024

Covington MF, Panda S, Liu XL, Strayer CA, Wagner DR, Kay SA (2001) ELF3 Modulates Resetting of the Circadian Clock in Arabidopsis. The Plant Cell 13: 13051316

Curie C, Alonso JM, Le Jean M, Ecker JR, Briat JF (2000) Involvement of NRAMP1 from Arabidopsis thaliana in iron transport. Biochemical Journal 347: 749-755 
Curie C, Cassin G, Couch D, Divol F, Higuchi K, Le Jean M, Misson J, Schikora A, Czernic P, Mari S (2009) Metal movement within the plant: Contribution of nicotianamine and yellow stripe 1-like transporters. Annals of Botany 103: 1-11

de Abreu-Neto JB, Turchetto-Zolet AC, de Oliveira LFV, Bodanese Zanettini MH, Margis-Pinheiro M (2013) Heavy metal-associated isoprenylated plant protein (HIPP): characterization of a family of proteins exclusive to plants. FEBS Journal 280: 16041616

De Bruyne L, Höfte M, De Vleesschauwer D (2014) Connecting Growth and Defense: The Emerging Roles of Brassinosteroids and Gibberellins in Plant Innate Immunity. Molecular Plant 7: 943-959

De Clercq I, Vermeirssen V, Van Aken O, Vandepoele K, Murcha MW, Law SR, Inzé A, Ng S, Ivanova A, Rombaut D, van de Cotte B, Jaspers P, Van de Peer Y, Kangasjärvi J, Whelan J, Van Breusegem F (2013) The Membrane-Bound NAC Transcription Factor ANAC013 Functions in Mitochondrial Retrograde Regulation of the Oxidative Stress Response in Arabidopsis. The Plant Cell 25: 3472-3490

de Folter S, Immink RGH (2011) Yeast Protein-Protein Interaction Assays and Screens. In L Yuan, SE Perry, eds, Plant Transcription Factors: Methods and Protocols. Humana Press, Totowa, NJ, pp 145-165

de Folter S, Immink RG H, K ieffer M, Pařenicová L, H enz SR, Weigel D, Busscher M, Kooiker M, Colombo L, Kater MM, Davies B, Angenent GC (2005) Comprehensive Interaction Map of the Arabidopsis MADS Box Transcription Factors. The Plant Cell 17: $1424-1433$

de Nadal E, Ammerer G, Posas F (2011) Controlling gene expression in response to stress. 12: $833-845$

Deinlein U, Weber M, Schmidt H, Rensch S, Trampczynska A, Hansen TH, Husted S, Schjoerring JK, Talke IN, Krämer U, Clemens S (2012) Elevated Nicotianamine Levels in Arabidopsis halleri Roots Play a Key Role in Zinc Hyperaccumulation. The Plant Cell 24: 708-723

Des Marais DL, Juenger TE (2010) Pleiotropy, plasticity, and the evolution of plant abiotic stress tolerance. Annals of the New York Academy of Sciences 1206: 56-79

Desbrosses-Fonrouge AG, Voigt K, Schröder A, Arrivault S, Thomine S, Krämer U (2005) Arabidopsis thaliana MTP1 is a $\mathrm{Zn}$ transporter in the vacuolar membrane which mediates $\mathrm{Zn}$ detoxification and drives leaf $\mathrm{Zn}$ accumulation. FEBS Letters 579: 41654174 
Diaz-Mendoza M, Velasco-Arroyo B, Santamaria ME, González-Melendi P, Martinez M, Diaz I (2016) Plant senescence and proteolysis: two processes with one destiny. Genetics and Molecular Biology 39: 329-338

Djamei A, Pitzschke A, Nakagami H, Rajh I, Hirt H (2007) Trojan Horse Strategy in Agrobacterium Transformation: Abusing MAPK Defense Signaling. Science 318: 453 456

Dobin A, Davis CA, Schlesinger F, Drenkow J, Zaleski C, Jha S, Batut P, Chaisson M, Gingeras TR (2013) STAR: ultrafast universal RNA-seq aligner. Bioinformatics 29: 15-21

Dosztányi Z, Mészáros B, Simon I (2009) ANCHOR: web server for predicting protein binding regions in disordered proteins. Bioinformatics 25: 2745-2746

Dräger DB, Desbrosses-Fonrouge AG, Krach C, Chardonnens AN, Meyer RC, SaumitouLaprade P, Krämer U (2004) Two genes encoding Arabidopsis halleri MTP1 metal transport proteins co-segregate with zinc tolerance and account for high MTP1 transcript levels. Plant Journal 39: 425-439

Drevensek S, Goussot M, Duroc Y, Christodoulidou A, Steyaert S, Schaefer E, Duvernois E, Grandjean O, Vantard M, Bouchez D, Pastuglia M (2012) The Arabidopsis TRM1-TON1 Interaction Reveals a Recruitment Network Common to Plant Cortical Microtubule Arrays and Eukaryotic Centrosomes. The Plant Cell 24: 178-191

Dubeaux G, Neveu J, Zelazny E, Vert G (2018) Metal Sensing by the IRT1 TransporterReceptor Orchestrates Its Own Degradation and Plant Metal Nutrition. Molecular Cell 69: $953-964 . e 955$

Dunker AK, Lawson JD, Brown CJ, Williams RM, Romero P, Oh JS, Oldfield CJ, Campen AM, Ratliff CM, Hipps KW, Ausio J, Nissen MS, Reeves R, Kang C, Kissinger CR, Bailey RW, Griswold MD, Chiu W, Garner EC, Obradovic Z (2001) Intrinsically disordered protein. Journal of Molecular Graphics and Modelling 19: 2659

Durrett TP, Gassmann W, Rogers EE (2007) The FRD3-Mediated Efflux of Citrate into the Root Vasculature Is Necessary for Efficient Iron Translocation. Plant Physiology 144: 197-205

Dykema PE, Sipes PR, Marie A, Biermann BJ, Crowell DN, Randall SK (1999) A new class of proteins capable of binding transition metals. Plant Molecular Biology 41: 139150 
Eide D, Broderius M, Fett J, Guerinot ML (1996) A novel iron-regulated metal transporter from plants identified by functional expression in yeast. Proceedings of the National Academy of Sciences of the United States of America 93: 5624-5628

Eide DJ (2006) Zinc transporters and the cellular trafficking of zinc. Biochimica et Biophysica Acta - Molecular Cell Research 1763: 711-722

Eide DJ (2009) Homeostatic and Adaptive Responses to Zinc Deficiency in Saccharomyces cerevisiae. The Journal of Biological Chemistry 284: 18565-18569

Eren E, Argüello JM (2004) Arabidopsis HMA2, a divalent heavy metal-transporting P(IB)type ATPase, is involved in cytoplasmic Zn2+ homeostasis. Plant Physiology 136: $3712-3723$

Essigmann B, Güler S, Narang RA, Linke D, Benning C (1998) Phosphate availability affects the thylakoid lipid composition and the expression of SQD1, a gene required for sulfolipid biosynthesis in Arabidopsis thaliana. Proceedings of the National Academy of Sciences 95: 1950-1955

Evans GW (1986) Zinc and its deficiency diseases. Clinical Physiology and Biochemistry 4: 94-98

Evrard A, Bargmann BOR, Birnbaum KD, Tester M, Baumann U, Johnson AAT (2012) Fluorescence-Activated Cell Sorting for Analysis of Cell Type-Specific Responses to Salinity Stress in Arabidopsis and Rice. Methods in molecular biology (Clifton, N.J.) 913: $265-276$

Feeney KA, Hansen LL, Putker M, Olivares-Yañez C, Day J, Eades LJ, Larrondo LF, Hoyle NP, O'Neill JS, van Ooijen G (2016) Daily magnesium fluxes regulate cellular timekeeping and energy balance. Nature 532: 375-379

Ferrández-Ayela A, Micol-Ponce R, Sánchez-García AB, Alonso-Peral MM, Micol JL, Ponce MR (2013) Mutation of an Arabidopsis NatB N-Alpha-Terminal Acetylation Complex Component Causes Pleiotropic Developmental Defects. PLOS ONE 8: e80697

Fisher RA (1919) XV.- The Correlation between Relatives on the Supposition of Mendelian Inheritance. Transactions of the Royal Society of Edinburgh 52: 399-433

Fitter DW, Martin DJ, Copley MJ, Scotland RW, Langdale JA (2002) GLK gene pairs regulate chloroplast development in diverse plant species. The Plant Journal 31: 713727 
Floris M, Mahgoub H, Lanet E, Robaglia C, Menand B (2009) Post-transcriptional Regulation of Gene Expression in Plants during Abiotic Stress. International Journal of Molecular Sciences 10: 3168-3185

Fong JH, Shoemaker BA, Garbuzynskiy SO, Lobanov MY, Galzitskaya OV, Panchenko AR (2009) Intrinsic Disorder in Protein Interactions: Insights From a Comprehensive Structural Analysis. PLOS Computational Biology 5: e1000316

Fournier-Level A, Korte A, Cooper MD, Nordborg M, Schmitt J, Wilczek AM (2011) A map of local adaptation in Arabidopsis thaliana. Science 334: 86-89

Franks SJ (2011) Plasticity and evolution in drought avoidance and escape in the annual plant Brassica rapa. New Phytologist 190: 249-257

Friso G, van Wijk KJ (2015) Posttranslational Protein Modifications in Plant Metabolism. Plant Physiology 169: 1469-1487

Gabriel KR (1971) The Biplot Graphic Display of Matrices with Application to Principal Component Analysis. Biometrika 58: 453-467

Gagne JM, Smalle J, Gingerich DJ, Walker JM, Yoo S-D, Yanagisawa S, Vierstra RD (2004) Arabidopsis EIN3-binding F-box 1 and 2 form ubiquitin-protein ligases that repress ethylene action and promote growth by directing EIN3 degradation. Proceedings of the National Academy of Sciences of the United States of America 101: 6803-6808

Galbraith DW (2014) Flow cytometry and sorting in Arabidopsis. Methods in molecular biology (Clifton, N.J.) 1062: 509-537

Galea CA, Wang Y, Sivakolundu SG, Kriwacki RW (2008) Regulation of Cell Division by Intrinsically Unstructured Proteins; Intrinsic Flexibility, Modularity and Signaling Conduits. Biochemistry 47: 7598-7609

Gao W, Xiao S, Li H-Y, Tsao S-W, Chye M-L (2009) Arabidopsis thaliana acyl-CoA-binding protein ACBP2 interacts with heavy-metal-binding farnesylated protein AtFP6. New Phytologist 181: 89-102

García MJ, Lucena C, Romera FJ, Alcántara E, Pérez-Vicente R (2010) Ethylene and nitric oxide involvement in the up-regulation of key genes related to iron acquisition and homeostasis in Arabidopsis. Journal of Experimental Botany 61: 3885-3899

Gasber A, Klaumann S, Trentmann O, Trampczynska A, Clemens S, Schneider S, Sauer N, Feifer I, Bittner F, Mendel RR, Neuhaus HE (2011) Identification of an Arabidopsis solute carrier critical for intracellular transport and inter-organ allocation of molybdate. Plant Biology 13: 710-718

Geldner N (2013) Casparian strips. Current Biology 23: R1025-R1026 
Ghandilyan A, Barboza L, Tisné S, Granier C, Reymond M, Koornneef M, Schat H, Aarts MGM (2009) Genetic analysis identifies quantitative trait loci controlling rosette mineral concentrations in Arabidopsis thaliana under drought. New Phytologist 184: $180-192$

Ghandilyan A, Kutman UB, Kutman BY, Cakmak I, Aarts MGM (2012) Genetic analysis of the effect of zinc deficiency on Arabidopsis growth and mineral concentrations. Plant and Soil 361: 227-239

Ghandilyan A, Vreugdenhil D, Aarts MGM (2006) Progress in the genetic understanding of plant iron and zinc nutrition. Physiologia Plantarum 126: 407-417

Gibbs DJ (2015) Emerging Functions for N-Terminal Protein Acetylation in Plants. Trends in Plant Science 20: 599-601

Gibson G (2010) Hints of hidden heritability in GWAS. 42: 558-560

Giedroc DP, Arunkumar AI (2007) Metal sensor proteins: nature's metalloregulated allosteric switches. Dalton Transactions: $3107-3120$

Gilkerson J, Perez-Ruiz JM, Chory J, Callis J (2012) The plastid-localized pfkB-type carbohydrate kinases FRUCTOKINASE-LIKE 1 and 2 are essential for growth and development of Arabidopsis thaliana. BMC Plant Biology 12: 102

Gómez-Mena C, de Folter S, Costa MMR, Angenent GC, Sablowski R (2005) Transcriptional program controlled by the floral homeotic gene AGAMOUS during early organogenesis. Development 132: 429-438

Graham RD (2008) Micronutrient Deficiencies in Crops and Their Global Significance. In BJ Alloway, ed, Micronutrient Deficiencies in Global Crop Production. Springer Netherlands, Dordrecht, pp 41-61

Grotz N, Fox T, Connolly E, Park W, Guerinot ML, Eide D (1998) Identification of a family of zinc transporter genes from Arabidopsis that respond to zinc deficiency. Proceedings of the National Academy of Sciences of the United States of America 95: 7220-7224

Grotz N, Guerinot ML (2006) Molecular aspects of $\mathrm{Cu}$, Fe and $\mathrm{Zn}$ homeostasis in plants. Biochimica et Biophysica Acta - Molecular Cell Research 1763: 595-608

Grundy J, Stoker C, Carré IA (2015) Circadian regulation of abiotic stress tolerance in plants. Frontiers in Plant Science 6

Guerra AJ, Giedroc DP (2012) Metal site occupancy and allosteric switching in bacterial metal sensor proteins. Archives of Biochemistry and Biophysics 519: 210-222

Gusev A, Bhatia G, Zaitlen N, Vilhjalmsson BJ, Diogo D, Stahl EA, Gregersen PK, Worthington J, Klareskog L, Raychaudhuri S, Plenge RM, Pasaniuc B, Price AL 
(2013) Quantifying Missing Heritability at Known GWAS Loci. PLOS Genetics 9: e1003993

Gustin. J, E. LM, Donggiun K, Gunnam N, Marina T, E. SD (2009) MTP1-dependent Zn sequestration into shoot vacuoles suggests dual roles in $\mathrm{Zn}$ tolerance and accumulation in Zn-hyperaccumulating plants. The Plant Journal 57: 1116-1127

Hacisalihoglu G, Kochian LV (2003) How do some plants tolerate low levels of soil zinc? Mechanisms of zinc efficiency in crop plants. New Phytologist 159: 341-350

Haina Z, Uwe S (2017) Differences and commonalities of plant responses to single and combined stresses. The Plant Journal 90: 839-855

Hausmann A, Aguilar Netz DJ, Balk J, Pierik AJ, Mühlenhoff U, Lill R (2005) The eukaryotic P loop NTPase Nbp35: An essential component of the cytosolic and nuclear iron-sulfur protein assembly machinery. Proceedings of the National Academy of Sciences of the United States of America 102: 3266-3271

Haydon MJ, Bell LJ, Webb AAR (2011) Interactions between plant circadian clocks and solute transport. Journal of Experimental Botany 62: 2333-2348

Haydon MJ, Cobbett CS (2007) Transporters of ligands for essential metal ions in plants. New Phytologist 174: 499-506

Haydon MJ, Román Á, Arshad W (2015) Nutrient homeostasis within the plant circadian network. Frontiers in Plant Science 6: 299

He L, Su C, Wang Y, Wei Z (2015) ATDOF5.8 protein is the upstream regulator of ANAC069 and is responsive to abiotic stress. Biochimie 110: 17-24

Henriques R, Jásik J, Klein M, Martinoia E, Feller U, Schell J, Pais MS, Koncz C (2002) Knock-out of Arabidopsis metal transporter gene IRT1 results in iron deficiency accompanied by cell differentiation defects. Plant Molecular Biology 50: 587-597

Hermans C, Conn SJ, Chen J, Xiao Q, Verbruggen N (2013) An update on magnesium homeostasis mechanisms in plants. Metallomics 5: 1170-1183

Himelblau E, Amasino RM (2001) Nutrients mobilized from leaves of Arabidopsis thaliana during leaf senescence. Journal of Plant Physiology 158: 1317-1323

Hindt MN, Guerinot ML (2012) Getting a sense for signals: regulation of the plant iron deficiency response. Biochimica et biophysica acta 1823: 1521-1530

Ho C-H, Lin S-H, Hu H-C, Tsay Y-F (2009) CHL1 Functions as a Nitrate Sensor in Plants. Cell 138: 1184-1194 
Hong S, Kim SA, Guerinot ML, McClung CR (2013) Reciprocal Interaction of the Circadian Clock with the Iron Homeostasis Network in Arabidopsis. Plant Physiology 161: 893903

Horstman A, Fukuoka H, Muino Acuna JM, Nitsch LMC, Guo C, Passarinho PA, Sanchez Perez GF, Immink RGH, Angenent GC, Boutilier KA (2015) AIL and HDG proteins act antagonistically to control cell proliferation. In, Vol 142. Development, pp 454-464

Horton MW, Hancock AM, Huang YS, Toomajian C, Atwell S, Auton A, Muliyati NW, Platt A, Sperone FG, Vilhjalmsson BJ, Nordborg M, Borevitz JO, Bergelson J (2012) Genome-wide patterns of genetic variation in worldwide Arabidopsis thaliana accessions from the RegMap panel. 44: 212-216

Hruz T, Laule O, Szabo G, Wessendorp F, Bleuler S, Oertle L, Widmayer P, Gruissem W, Zimmermann P (2008) Genevestigator V3: A Reference Expression Database for the Meta-Analysis of Transcriptomes. Advances in Bioinformatics 2008: 5

Huang C, Barker SJ, Langridge P, Smith FW, Graham RD (2000) Zinc Deficiency UpRegulates Expression of High-Affinity Phosphate Transporter Genes in Both Phosphate-Sufficient and -Deficient Barley Roots. Plant Physiology 124: 415-422

Huang DW, Sherman BT, Lempicki RA (2008) Systematic and integrative analysis of large gene lists using DAVID bioinformatics resources. 4: 44

Huang DW, Sherman BT, Lempicki RA (2009) Bioinformatics enrichment tools: paths toward the comprehensive functional analysis of large gene lists. Nucleic Acids Research 37: 1-13

Huang X-Y, Salt David E (2016) Plant Ionomics: From Elemental Profiling to Environmental Adaptation. Molecular Plant 9: 787-797

Huot B, Yao J, Montgomery BL, He SY (2014) Growth-Defense Tradeoffs in Plants: A Balancing Act to Optimize Fitness. Molecular Plant 7: 1267-1287

Hurst LD, Pál C, Lercher MJ (2004) The evolutionary dynamics of eukaryotic gene order. 5: 299

Hussain D, Haydon MJ, Wang Y, Wong E, Sherson SM, Young J, Camakaris J, Harper JF, Cobbett CS (2004) P-type ATPase heavy metal transporters with roles in essential zinc homeostasis in arabidopsis. Plant Cell 16: 1327-1339

Iakoucheva LM, Brown CJ, Lawson JD, O bradović Z, Dunker AK (2002) Intrinsic Disorder in Cell-signaling and Cancer-associated Proteins. Journal of Molecular Biology 323: 573-584 
Immink RGH, Tonaco IAN, de Folter S, Shchennikova A, van Dijk ADJ, Busscher-Lange J, Borst JW, Angenent GC (2009) SEPALLATA3: the 'glue' for MADS box transcription factor complex formation. Genome Biology 10: R24-R24

Ishijima S, Shigemi Z, Adachi H, Makinouchi N, Sagami I (2012) Functional reconstitution and characterization of the Arabidopsis Mg2+ transporter AtMRS2-10 in proteoliposomes. Biochimica et Biophysica Acta (BBA) - Biomembranes 1818: 2202 2208

Jammes F, Lecomte P, de Almeida-Engler J, Bitton F, Martin-Magniette M-L, Renou JP, Abad P, Favery B (2005) Genome-wide expression profiling of the host response to root-knot nematode infection in Arabidopsisa. The Plant Journal 44: 447-458

Jin J, Xie X, Chen C, Park JG, Stark C, James DA, Olhovsky M, Linding R, Mao Y, Pawson T (2009) Eukaryotic Protein Domains as Functional Units of Cellular Evolution. Science Signaling 2: ra76-ra76

Johnson C, Knight M, Kondo T, Masson P, Sedbrook J, Haley A, Trewavas A (1995) Circadian oscillations of cytosolic and chloroplastic free calcium in plants. Science 269: 1863-1865

Jung J-H, Lee S, Yun J, Lee M, Park C-M (2014) The miR172 target TOE3 represses AGAMOUS expression during Arabidopsis floral patterning. Plant Science 215-216: 29-38

Kammler M, Schön C, Hantke K (1993) Characterization of the ferrous iron uptake system of Escherichia coli. Journal of Bacteriology 175: 6212-6219

Karve TM, Cheema AK (2011) Small Changes Huge Impact: The Role of Protein Posttranslational Modifications in Cellular Homeostasis and Disease. Journal of Amino Acids 2011

Kasai K, Takano J, Miwa K, Toyoda A, Fujiwara T (2011) High Boron-induced Ubiquitination Regulates Vacuolar Sorting of the BOR1 Borate Transporter in Arabidopsis thaliana. Journal of Biological Chemistry 286: 6175-6183

Kasajima I, Fujiwara T (2007) Identification of novel Arabidopsis thaliana genes which are induced by high levels of boron. Plant Biotechnology 24: 355-360

Kasuga M, Liu Q, Miura S, Yamaguchi-Shinozaki K, Shinozaki K (1999) Improving plant drought, salt, and freezing tolerance by gene transfer of a single stress-inducible transcription factor. Nature Biotechnology 17: 287

Kazan K, Lyons R (2016) The link between flowering time and stress tolerance. Journal of Experimental Botany 67: 47-60 
Kessler D, Papenbrock J (2005) Iron-sulfur cluster biosynthesis in photosynthetic organisms. Photosynthesis Research 86: 391-407

Kim HS, Park BO, Yoo JH, Jung MS, Lee SM, Han HJ, Kim KE, Kim SH, Lim CO, Yun D-J, Lee SY, Chung WS (2007) Identification of a Calmodulin-binding NAC Protein as a Transcriptional Repressor in Arabidopsis. Journal of Biological Chemistry 282: 36292-36302

Kim K-C, Lai Z, Fan B, Chen Z (2008) Arabidopsis WRKY38 and WRKY62 Transcription Factors Interact with Histone Deacetylase 19 in Basal Defense. The Plant Cell 20: 23572371

Kim S-G, Kim S-Y, Park C-M (2007) A membrane-associated NAC transcription factor regulates salt-responsive flowering via FLOWERING LOCUS T in Arabidopsis. Planta 226: $647-654$

Kim S-G, Lee A-K, Yoon H-K, Park C-M (2008) A membrane-bound NAC transcription factor NTL8 regulates gibberellic acid-mediated salt signaling in Arabidopsis seed germination. The Plant Journal 55: 77-88

Kim S, Plagnol V, Hu TT, Toomajian C, Clark RM, Ossowski S, Ecker JR, Weigel D, Nordborg M (2007) Recombination and linkage disequilibrium in Arabidopsis thaliana. 39: 1151-1155

Kim W-Y, Ali Z, Park HJ, Park SJ, Cha J-Y, Perez-Hormaeche J, Quintero FJ, Shin G, Kim MR, Qiang Z, Ning L, Park HC, Lee SY, Bressan RA, Pardo JM, Bohnert HJ, Yun D-J (2013) Release of SOS2 kinase from sequestration with GIGANTEA determines salt tolerance in Arabidopsis. Nature Communications 4: 1352

Kim Y-S, Sakuraba Y, Han S-H, Yoo S-C, Paek N-C (2013) Mutation of the Arabidopsis NAC016 Transcription Factor Delays Leaf Senescence. Plant and Cell Physiology 54: $1660-1672$

Klein P, Seidel T, Stöcker B, Dietz K-J (2012) The membrane-tethered transcription factor ANAC089 serves as redox-dependent suppressor of stromal ascorbate peroxidase gene expression. Frontiers in Plant Science 3: 247

Knoth C, Eulgem T (2008) The oomycete response gene LURP1 is required for defense against Hyaloperonospora parasitica in Arabidopsis thaliana. The Plant Journal 55: 5364

Kobae Y, Uemura T, Sato MH, Ohnishi M, Mimura T, Nakagawa T, Maeshima M (2004) Zinc transporter of Arabidopsis thaliana AtMTP1 is localized to vacuolar membranes and implicated in zinc homeostasis. Plant and Cell Physiology 45: 1749-1758 
Konstantinopoulos PA, Karamouzis MV, Papavassiliou AG (2007) Post-translational modifications and regulation of the RAS superfamily of GTPases as anticancer targets. Nature Reviews Drug Discovery 6: 541

Koornneef M, Alonso-Blanco C, Vreugdenhil D (2004) Naturally ocurring genetic variation in Arabidopsis thaliana Annual Review of Plant Biology 55: 141-172

Koornneef M, Meinke D (2010) The development of Arabidopsis as a model plant. The Plant Journal 61: 909-921

Korshunova YO, Eide D, Clark WG, Guerinot ML, Pakrasi HB (1999) The IRT1 protein from Arabidopsis thaliana is a metal transporter with a broad substrate range. Plant Molecular Biology 40: 37-44

Korshunova YO, Eide D, Gregg Clark W, Lou Guerinot M, Pakrasi HB (1999) The IRT1 protein from Arabidopsis thaliana is a metal transporter with a broad substrate range. Plant Molecular Biology 40: 37-44

Korte A, Farlow A (2013) The advantages and limitations of trait analysis with GWAS: a review. Plant Methods 9: 29

Korves TM, Bergelson J (2003) A Developmental Response to Pathogen Infection in Arabidopsis. Plant Physiology 133: 339-347

Kovinich N, Kayanja G, Chanoca A, Riedl K, Otegui MS, Grotewold E (2014) Not all anthocyanins are born equal: distinct patterns induced by stress in Arabidopsis. Planta 240: $931-940$

Kremers G-J, Goedhart J, van Munster EB, Gadella TWJ (2006) Cyan and Yellow Super Fluorescent Proteins with Improved Brightness, Protein Folding, and FRET Förster Radius. Biochemistry 45: 6570-6580

K rężel A, Maret W(2006) Zinc-buffering capacity of a eukaryotic cell at physiological pZn. JBIC Journal of Biological Inorganic Chemistry 11: 1049-1062

K rężel A, Maret W (2016) The biological inorganic chemistry of zinc ions. Archives of Biochemistry and Biophysics 611: 3-19

Kruijer W, Boer MP, Malosetti M, Flood PJ, Engel B, Kooke R, Keurentjes JJB, van Eeuwijk FA (2015) Marker-Based Estimation of Heritability in Immortal Populations. Genetics 199: 379-398

Kuepper H, Fang Jie Z, McGrath SP (1999) Cellular Compartmentation of Zinc in Leaves of the Hyperaccumulator Thlaspi caerulescens. PLANT PHYSIOLOGY -ROCKVILLE PIKE BETHESDA- 119: 305-311 
Kwon K-C, Cho MH (2008) Deletion of the chloroplast-localized AtTerC gene product in Arabidopsis thaliana leads to loss of the thylakoid membrane and to seedling lethality. The Plant Journal 55: 428-442

la Rosa NM-d, Sotillo B, Miskolczi P, Gibbs DJ, Vicente J, Carbonero P, Oñate-Sánchez L, Holdsworth MJ, Bhalerao R, Alabadí D, Blázquez MA (2014) Large-Scale Identification of Gibberellin-Related Transcription Factors Defines Group VII ETHYLENE RESPONSE FACTORS as Functional DELLA Partners. Plant Physiology 166: $1022-1032$

Law CW, Chen Y, Shi W, Smyth GK (2014) voom: precision weights unlock linear model analysis tools for RNA-seq read counts. Genome Biology 15: R29

Lee J-W, Helmann JD (2007) Functional specialization within the Fur family of metalloregulators. BioMetals 20: 485

Lee M-H, Jeon HS, Kim HG, Park OKC (2017) An Arabidopsis NAC transcription factor NAC4 promotes pathogen-induced cell death under negative regulation by microRNA164. New Phytologist 214: 343-360

Li H-Y, Chye M-L (2004) Arabidopsis Acyl-CoA-Binding Protein ACBP2 Interacts With an Ethylene-Responsive Element-Binding Protein, AtEBP, via its Ankyrin Repeats. Plant Molecular Biology 54: 233-243

Li J, Zhang J, Wang X, Chen J (2010) A membrane-tethered transcription factor ANAC089 negatively regulates floral initiation in Arabidopsis thaliana. Science China Life Sciences 53: 1299-1306

Li L, Tutone AF, Revel SMD, Gardner RC, Luan S (2001) A Novel Family of Magnesium Transport Genes in Arabidopsis. The Plant Cell 13: 2761-2775

Li X, Zhang H, Ai Q, Liang G, Yu D (2016) Two bHLH Transcription Factors, bHLH34 and bHLH104, Regulate Iron Homeostasis in Arabidopsis thaliana. Plant Physiology 170: $2478-2493$

Li Y, Huang Y, Bergelson J, Nordborg M, Borevitz JO (2010) Association mapping of local climate-sensitive quantitative trait loci in Arabidopsis thaliana. Proceedings of the National Academy of Sciences 107: 21199-21204

Liang G, Zhang H, Li X, Ai Q, Yu D (2017) bHLH transcription factor bHLH115 regulates iron homeostasis in Arabidopsis thaliana. Journal of Experimental Botany 68: 17431755 
Lin XY, Ye YQ, Fan SK, Jin CW, Zheng SJ (2016) Increased Sucrose Accumulation Regulates Iron-Deficiency Responses by Promoting Auxin Signaling in Arabidopsis Plants. Plant Physiology 170: 907-920

Lin Y-F, Hassan Z, Talukdar S, Schat H, Aarts MGM (2016) Expression of the ZNT1 Zinc Transporter from the Metal Hyperaccumulator Noccaea caerulescens Confers Enhanced Zinc and Cadmium Tolerance and Accumulation to Arabidopsis thaliana. PLoS ONE 11: e0149750

Lin Y-L, Tsay Y-F (2017) Influence of differing nitrate and nitrogen availability on flowering control in Arabidopsis. Journal of Experimental Botany 68: 2603-2609

Lin YF, Liang HM, Yang SY, Boch A, Clemens S, Chen CC, Wu JF, Huang JL, Yeh KC (2009) Arabidopsis IRT3 is a zinc-regulated and plasma membrane localized zinc/iron transporter. New Phytologist 182: 392-404

Linster E, Stephan I, Bienvenut WV, Maple-Grødem J, Myklebust LM, Huber M, Reichelt M, Sticht C, Møller SG, Meinnel T, Arnesen T, Giglione C, Hell R, Wirtz M (2015) Downregulation of N-terminal acetylation triggers ABA-mediated drought responses in Arabidopsis. Nature Communications 6

Liszczak G, Goldberg JM, Foyn H, Petersson EJ, Arnesen T, Marmorstein R (2013) Molecular Basis for Amino-Terminal Acetylation by the Heterodimeric NatA Complex. Nature structural \& molecular biology 20: 1098-1105

Liu W, Karemera NJU, Wu T, Yang Y, Zhang X, Xu X, Wang Y, Han Z (2017) The ethylene response factor AtERF4 negatively regulates the iron deficiency response in Arabidopsis thaliana. PLOS ONE 12: e 0186580

Liu W, Xie Y, Ma J, Luo X, Nie P, Zuo Z, Lahrmann U, Zhao Q, Zheng Y, Zhao Y, Xue Y, Ren J (2015) IBS: an illustrator for the presentation and visualization of biological sequences. Bioinformatics 31: 3359-3361

Liu Y, Ji X, Nie X, Qu M, Zheng L, Tan Z, Zhao H, Huo L, Liu S, Zhang B, Wang YC (2015) Arabidopsis AtbHLH112 regulates the expression of genes involved in abiotic stress tolerance by binding to their E-box and GCG-box motifs. New Phytologist 207: 692-709

Liu Y, Xie Y, Wang H, Ma X, Yao W, Wang H (2017) Light and Ethylene Coordinately Regulate the Phosphate Starvation Response through Transcriptional Regulation of PHOSPHATE STARVATION RESPONSE1. The Plant Cell 29: 2269-2284 
Long Y, Stahl Y, Weidtkamp-Peters S, Postma M, Zhou W, Goedhart J, Sánchez-Pérez M-I, Gadella TWJ, Simon R, Scheres B, Blilou I (2017) In vivo FRET-FLIM reveals cell-type-specific protein interactions in Arabidopsis roots. Nature 548: 97

Lopez-Maury L, Marguerat S, Bahler J (2008) Tuning gene expression to changing environments: from rapid responses to evolutionary adaptation. 9: 583-593

Loqué D, Ludewig U, Yuan L, von Wirén N (2005) Tonoplast Intrinsic Proteins AtTIP2;1 and AtTIP2;3 Facilitate NH3 Transport into the Vacuole. Plant Physiology 137: 671680

Lozano-Durán R, Zipfel C (2015) Trade-off between growth and immunity: role of brassinosteroids. Trends in Plant Science 20: 12-19

Lucena C, Waters BM, Romera FJ, García MJ, Morales M, Alcántara E, Pérez-Vicente R (2006) Ethylene could influence ferric reductase, iron transporter, and H+-ATPase gene expression by affecting FER (or FER-like) gene activity. Journal of Experimental Botany 57: 4145-4154

Mach J (2012) Plant Cortical Microtubule Arrays: Recruitment Mechanisms in Common with Centrosomes. The Plant Cell 24: 2-2

Maillard A, Diquélou S, Billard V, Laîné P, Garnica M, Prudent M, Garcia-Mina J-M, Yvin J-C, Ourry A (2015) Leaf mineral nutrient remobilization during leaf senescence and modulation by nutrient deficiency. Frontiers in Plant Science 6

Manolio TA, Collins FS, Cox NJ, Goldstein DB, Hindorff LA, Hunter DJ, McCarthy MI, Ramos EM, Cardon LR, Chakravarti A, Cho JH, Guttmacher AE, Kong A, Kruglyak L, Mardis E, Rotimi CN, Slatkin M, Valle D, Whittemore AS, Boehnke M, Clark AG, Eichler EE, Gibson G, Haines JL, Mackay TFC, McCarroll SA, Visscher PM (2009) Finding the missing heritability of complex diseases. Nature 461: $747-753$

Mao Y-B, Xue X-Y, Tao X-Y, Yang C-Q, Wang L-J, Chen X-Y (2013) Cysteine protease enhances plant-mediated bollworm RNA interference. Plant Molecular Biology 83: $119-129$

Marjoram P, Zubair A, Nuzhdin SV (2014) Post-GWAS: where next? More samples, more SNPs or more biology? Heredity 112: 79-88

Marschner H, Marschner P (2012) Marschner's mineral nutrition of higher plants. Academic Press, London

Martínez-Medina A, Van Wees SCM, Pieterse CMJCPCER (2017) Airborne signals from Trichoderma fungi stimulate iron uptake responses in roots resulting in priming of 
jasmonic acid-dependent defences in shoots of Arabidopsis thaliana and Solanum lycopersicum. Plant, Cell \& Environment 40: 2691-2705

Mäser P, Thomine S, Schroeder JI, Ward JM, Hirschi K, Sze H, Talke IN, Amtmann A, Maathuis FJM, Sanders D, Harper JF, Tchieu J, Gribskov M, Persans MW, Salt DE, Kim SA, Guerinot ML (2001) Phylogenetic Relationships within Cation Transporter Families of Arabidopsis. Plant Physiology 126: 1646-1667

Mathilde S, Jean-François B, Grégory V, Catherine C (2008) Cytokinins negatively regulate the root iron uptake machinery in Arabidopsis through a growth-dependent pathway. The Plant Journal 55: 289-300

Mattiello EM, Ruiz HA, Neves JCL, Ventrella MC, Araújo WL (2015) Zinc deficiency affects physiological and anatomical characteristics in maize leaves. Journal of Plant Physiology 183: 138-143

Maurer-Stroh S, Eisenhaber F (2005) Refinement and prediction of protein prenylation motifs. Genome Biology 6: R55-R55

Medici A, Marshall-Colon A, Ronzier E, Szponarski W, Wang R, Gojon A, Crawford NM, Ruffel S, Coruzzi GM, Krouk G (2015) AtNIGT1/HRS1 integrates nitrate and phosphate signals at the Arabidopsis root tip. Nature communications 6: 6274-6274

Meier S, Bastian R, Donaldson L, Murray S, Bajic V, Gehring C (2008) Co-expression and promoter content analyses assign a role in biotic and abiotic stress responses to plant natriuretic peptides. BMC Plant Biology 8: 24

Meijón M, Satbhai SB, Tsuchimatsu T, Busch W (2014) Genome-wide association study using cellular traits identifies a new regulator of root development in Arabidopsis. Nature genetics 46: 77-81

Meinke DW, Cherry JM, Dean C, Rounsley SD, Koornneef M (1998) Arabidopsis thaliana: a model plant for genome analysis. Science (New York, N.Y.) 282: 662, 679-682

Meinke DW, Meinke LK, Showalter TC, Schissel AM, Mueller LA, Tzafrir I (2003) A Sequence-Based Map of Arabidopsis Genes with Mutant Phenotypes. Plant Physiology 131: 409-418

Meyers BC, Kozik A, Griego A, Kuang H, Michelmore RW (2003) Genome-Wide Analysis of NBS-LRR-Encoding Genes in Arabidopsis. The Plant Cell 15: 809-834

Miller M, Song Q, Shi X, Juenger TE, Chen ZJ (2015) Natural variation in timing of stressresponsive gene expression predicts heterosis in intraspecific hybrids of Arabidopsis. Nature Communications 6: 7453 
Mills RF, Krijger GC, Baccarini PJ, Hall JL, Williams LE (2003) Functional expression of AtHMA4, a P1B-type ATPase of the $\mathrm{Zn} / \mathrm{Co} / \mathrm{Cd} / \mathrm{Pb}$ subclass. The Plant Journal 35: 164176

Milner MJ, Seamon J, Craft E, Kochian LV (2013) Transport properties of members of the ZIP family in plants and their role in Zn and Mn homeostasis. Journal of Experimental Botany 64: 369-381

Mitrophanov AY, Groisman EA (2008) Positive feedback in cellular control systems. BioEssays : news and reviews in molecular, cellular and developmental biology 30: $542-555$

Mittler R (2002) Oxidative stress, antioxidants and stress tolerance. Trends in Plant Science 7: 405-410

Montanini B, Blaudez D, Jeandroz S, Sanders D, Chalot M (2007) Phylogenetic and functional analysis of the Cation Diffusion Facilitator (CDF) family: improved signature and prediction of substrate specificity. BMC Genomics 8: 107

Moore RC, Grant SR, Purugganan MD (2005) Molecular Population Genetics of Redundant Floral-Regulatory Genes in Arabidopsis thaliana. Molecular Biology and Evolution 22: 91-103

Morel M, Crouzet J, Gravot A, Auroy P, Leonhardt N, Vavasseur A, Richaud P (2009) AtHMA3, a P1B-ATPase allowing $\mathrm{Cd} / \mathrm{Zn} / \mathrm{Co} / \mathrm{Pb}$ vacuolar storage in Arabidopsis. Plant Physiology 149: 894-904

Müller D, Schmitz G, Theres K (2006) Blind Homologous R2R3 Myb Genes Control the Pattern of Lateral Meristem Initiation in Arabidopsis. The Plant Cell 18: 586-597

Müller O, Krawinkel M (2005) Malnutrition and health in developing countries. CMAJ : Canadian Medical Association Journal 173: 279-286

Murgia I, Vigani G (2015) Analysis of Arabidopsis thaliana atfer4-1, atfh and atfer4-1/atfh mutants uncovers frataxin and ferritin contributions to leaf ionome homeostasis. Plant Physiology and Biochemistry 94: 65-72

Murmu J, Wilton M, Allard G, Pandeya R, Desveaux D, Singh J, Subramaniam R (2014) Arabidopsis GOLDEN2-LIKE (GLK) transcription factors activate jasmonic acid (JA)dependent disease susceptibility to the biotrophic pathogen Hyaloperonospora arabidopsidis, as well as JA-independent plant immunity against the necrotrophic pathogen Botrytis cinerea. Molecular Plant Pathology 15: 174-184 
Nakashima K, Takasaki H, Mizoi J, Shinozaki K, Yamaguchi-Shinozaki K (2012) NAC transcription factors in plant abiotic stress responses. Biochimica et Biophysica Acta (BBA) - Gene Regulatory Mechanisms 1819: 97-103

Nichols BA, Hopkins BG, Jolley VD, Webb BL, Greenwood BG, Buck JR (2012) Phosphorus and zinc interactions and their relationships with other nutrients in maize grown in chelator-buffered nutrient solution. Journal of Plant Nutrition 35: 123-141

Nie P, Li X, Wang S, Guo J, Zhao H, Niu D (2017) Induced Systemic Resistance against Botrytis cinerea by Bacillus cereus AR156 through a JA/ET- and NPR1-Dependent Signaling Pathway and Activates PAMP-Triggered Immunity in Arabidopsis. Frontiers in Plant Science 8

Nielsen FH (2012) History of Zinc in Agriculture. Advances in Nutrition 3: 783-789

Nishida S, Kato A, Tsuzuki C, Yoshida J, Mizuno T (2015) Induction of Nickel Accumulation in Response to Zinc Deficiency in Arabidopsis thaliana. International Journal of Molecular Sciences 16: 9420

Nolan TM, Brennan B, Yang M, Chen J, Zhang M, Li Z, Wang X, Bassham DC, Walley J, Yin Y (2017) Selective Autophagy of BES1 Mediated by DSK2 Balances Plant Growth and Survival. Developmental Cell 41: 33-46.e37

Nordborg M, Weigel D (2008) Next-generation genetics in plants. Nature 456: 720-723

O 'Shea C, K ryger M, Stender EmilGP, Kragelund Birthe B, Willemoës M, Skriver K (2015) Protein intrinsic disorder in Arabidopsis NAC transcription factors: transcriptional activation by ANAC013 and ANAC046 and their interactions with RCD1. Biochemical Journal 465: 281-294

Ogawa T, Nishimura K, Aoki T, Takase H, Tomizawa K-I, Ashida H, Yokota A (2009) A Phosphofructokinase B-Type Carbohydrate Kinase Family Protein, NARA5, for Massive Expressions of Plastid-Encoded Photosynthetic Genes in Arabidopsis. Plant Physiology 151: 114-128

Page DR, Grossniklaus U (2002) The art and design of genetic screens: Arabidopsis thaliana. Nature Reviews Genetics 3: 124

Pagni M, Ioannidis V, Cerutti L, Zahn-Zabal M, Jongeneel CV, Hau J, Martin O, Kuznetsov D, Falquet L (2007) MyHits: improvements to an interactive resource for analyzing protein sequences. Nucleic Acids Research 35: W433-W437

Palmer CM, Hindt MN, Schmidt H, Clemens S, Guerinot ML (2013) MYB10 and MYB72 Are Required for Growth under Iron-Limiting Conditions. PLoS Genetics 9: e1003953 
Pan IC, Tsai H-H, Cheng Y-T, Wen T-N, Buckhout TJ, Schmidt W (2015) PostTranscriptional Coordination of the Arabidopsis Iron Deficiency Response is Partially Dependent on the E3 Ligases RING DOMAIN LIGASE1 (RGLG1) and RING DOMAIN LIGASE2 (RGLG2). Molecular \& Cellular Proteomics : MCP 14: 27332752

Pang WL, Kaur A, Ratushny AV, Cvetkovic A, Kumar S, Pan M, Arkin AP, Aitchison JD, Adams MWW, Baliga NS (2013) Metallochaperones Regulate Intracellular Copper Levels. PLoS Computational Biology 9

Park J-S, Wang M, Park S-J, Lee S-H (1999) Zinc Finger of Replication Protein A, a NonDNA Binding Element, Regulates Its DNA Binding Activity through Redox. Journal of Biological Chemistry 274: 29075-29080

Park J, Kim Y-S, Kim S-G, Jung J-H, Woo J-C, Park C-M (2011) Integration of Auxin and Salt Signals by the NAC Transcription Factor NTM2 during Seed Germination in Arabidopsis. Plant Physiology 156: 537-549

Paulsen IT, Saier Jr MH (1997) A novel family of ubiquitous heavy metal ion transport proteins. Journal of Membrane Biology 156: 99-103

Pérez-Pérez JM, Candela H, Micol JL (2009) Understanding synergy in genetic interactions. Trends in Genetics 25: 368-376

Pérez-Pérez JM, Esteve-Bruna D, González-Bayón R, Kangasjärvi S, Caldana C, Hannah MA, Willmitzer L, Ponce MR, Micol JL (2013) Functional Redundancy and Divergence within the Arabidopsis RETICULATA-RELATED Gene Family. Plant Physiology 162: 589-603

Persson DP, Chen A, Aarts MGM, Salt DE, Schjoerring JK, Husted S (2016) Multi-element bioimaging of Arabidopsis thaliana roots. Plant Physiology 172: 835-847

Pesaresi P, Gardner NA, Masiero S, Dietzmann A, Eichacker L, Wickner R, Salamini F, Leister D (2003) Cytoplasmic N-Terminal Protein Acetylation Is Required for Efficient Photosynthesis in Arabidopsis. The Plant Cell 15: 1817

Petricka JJ, Clay NK, Nelson TM (2008) Vein patterning screens and the defectively organized tributaries mutants in Arabidopsis thaliana. The Plant Journal 56: 251-263

Pickett FB, Meeks-Wagner DR (1995) Seeing double: appreciating genetic redundancy. The Plant Cell 7: 1347-1356

Pineau C, Loubet S, Lefoulon C, Chalies C, Fizames C, Lacombe B, Ferrand M, Loudet O, Berthomieu P, Richard O (2012) Natural Variation at the FRD3 MATE Transporter 
Locus Reveals Cross-Talk between Fe Homeostasis and Zn Tolerance in Arabidopsis thaliana. PLoS Genetics 8

Polevoda B, Sherman F (2000) Na-terminal Acetylation of Eukaryotic Proteins. Journal of Biological Chemistry 275: 36479-36482

Polevoda B, Sherman F (2003) Composition and function of the eukaryotic N-terminal acetyltransferase subunits. Biochemical and Biophysical Research Communications 308: $1-11$

Prasad AS (2003) Zinc deficiency : Has been known of for 40 years but ignored by global health organisations. BMJ : British Medical Journal 326: 409-410

Prasad AS (2012) Discovery of human zinc deficiency: 50 years later. Journal of Trace Elements in Medicine and Biology 26: 66-69

Prasad AS, Miale A, Jr., Farid Z, Sandstead HH, Schulert AR (1963) Zinc Metabolism in Patients with the Syndrome of Iron Deficiency Anemia, Hepatosplenomegaly, Dwarfism, and Hypogonadism. Journal of Laboratory and Clinical Medicine 61: 537549

Printz B, Lutts S, Hausman J-F, Sergeant K (2016) Copper Trafficking in Plants and Its Implication on Cell Wall Dynamics. Frontiers in Plant Science 7

Provart NJ, Alonso J, Assmann SM, Bergmann D, Brady SM, Brkljacic J, Browse J, Chapple C, Colot V, Cutler S, Dangl J, Ehrhardt D, Friesner JD, Frommer WB, Grotewold E, Meyerowitz E, Nemhauser J, Nordborg M, Pikaard C, Shanklin J, Somerville C, Stitt M, Torii KU, Waese J, Wagner D, McCourt P (2016) 50 years of Arabidopsis research: Highlights and future directions. New Phytologist 209: 921944

Pruneda-Paz JL, Breton G, Nagel DH, Kang SE, Bonaldi K, Doherty CJ, Ravelo S, Galli M, Ecker JR, Kay SA (2014) A genome-scale resource for the functional characterization of Arabidopsis transcription factors. Cell reports 8: 622-632

Przedpelska-Wasowicz E, Wasowicz P (2013) Does zinc concentration in the substrate influence the onset of flowering in Arabidopsis arenosa (Brassicaceae)? Plant Growth Regulation 69: 87-97

Puig S, Peñarrubia L (2009) Placing metal micronutrients in context: transport and distribution in plants. Current Opinion in Plant Biology 12: 299-306

Ragel P, Ródenas R, García-Martín E, Andrés Z, Villalta I, Nieves-Cordones M, Rivero RM, Martínez V, Pardo JM, Quintero FJ, Rubio F (2015) The CBL-Interacting 
Protein Kinase CIPK23 Regulates HAK5-Mediated High-Affinity K(+) Uptake in Arabidopsis Roots. Plant Physiology 169: 2863-2873

Ravet K, Touraine B, Boucherez J, Briat J-F, Gaymard F, Cellier F (2009) Ferritins control interaction between iron homeostasis and oxidative stress in Arabidopsis. The Plant Journal 57: 400-412

Reed JW, Nagpal P, Bastow RM, Solomon KS, Dowson-Day MJ, Elumalai RP, Millar AJ (2000) Independent Action of ELF3 and phyB to Control Hypocotyl Elongation and Flowering Time. Plant Physiology 122: 1149-1160

Regalla LM, Lyons TJ (2006) Zinc in yeast: mechanisms involved in homeostasis. Topics in current genetics 14: $37-58$

Reyt G, Boudouf S, Boucherez J, Gaymard F, Briat J-F (2015) Iron- and Ferritin-Dependent Reactive Oxygen Species Distribution: Impact on Arabidopsis Root System Architecture. Molecular Plant 8: 439-453

Riboni M, Robustelli Test A, Galbiati M, Tonelli C, Conti L (2014) Environmental stress and flowering time: The photoperiodic connection. Plant Signaling \& Behavior 9: e29036

Riggs JW, Callis J (2016) Studies of the PfkB Family of Proteins in Arabidopsis thaliana. The FASEB Journal 30: 1164

Robinson NJ, Winge DR (2010) Copper metallochaperones. In Annual Review of Biochemistry, Vol 79, pp 537-562

Robinson WD, Carson I, Ying S, Ellis K, Plaxton WC (2012) Eliminating the purple acid phosphatase AtPAP26 in Arabidopsis thaliana delays leaf senescence and impairs phosphorus remobilization. New Phytologist 196: 1024-1029

Rockman MV (2012) The QNT program and the alleles that matter for evolution: all that's gold does not glitter Evolution 66: 1-17

Roohani N, Hurrell R, Kelishadi R, Schulin R (2013) Zinc and its importance for human health: An integrative review. Journal of Research in Medical Sciences : The Official Journal of Isfahan University of Medical Sciences 18: 144-157

Roskoski R (2003) Protein prenylation: a pivotal posttranslational process. Biochemical and Biophysical Research Communications 303: 1-7

Rueda-Romero P, Barrero-Sicilia C, Gómez-Cadenas A, Carbonero P, Oñate-Sánchez L (2012) Arabidopsis thaliana DOF6 negatively affects germination in non-after-ripened seeds and interacts with TCP14. Journal of Experimental Botany 63: 1937-1949 
Sakuraba Y, Han S-H, Lee S-H, Hörtensteiner S, Paek N-C (2016) Arabidopsis NAC016 promotes chlorophyll breakdown by directly upregulating STAYGREEN1 transcription. Plant Cell Reports 35: 155-166

Salt DE, Baxter I, Lahner B (2008) Ionomics and the Study of the Plant Ionome. Annual Review of Plant Biology 59: 709-733

Sanda S, Leustek T, Theisen MJ, Garavito RM, Benning C (2001) Recombinant Arabidopsis SQD1 Converts UDP-glucose and Sulfite to the Sulfolipid Head Group Precursor UDP-sulfoquinovose in Vitro. Journal of Biological Chemistry 276: 39413946

Sanz-Fernández M, Rodríguez-Serrano M, Sevilla-Perea A, Pena L, Mingorance MD, Sandalio LM, Romero-Puertas MC (2017) Screening Arabidopsis mutants in genes useful for phytoremediation. Journal of Hazardous Materials 335: 143-151

Sappl PG, Heisler MG (2013) Live-imaging of plant development: Latest approaches. Current Opinion in Plant Biology 16: 33-40

Schat H, Vooijs R, Kuiper E (1996) Identical major gene loci for heavy metal tolerances that have independently evolved in different local populations and subspecies of Silene vulgaris. Evolution 50: 1888-1895

Schlichting CD (1986) The Evolution of Phenotypic Plasticity in Plants. Annual Review of Ecology and Systematics 17: 667-693

Schmalenbach I, Zhang L, Reymond M, Jiménez-Gómez JM (2014) The relationship between flowering time and growth responses to drought in the Arabidopsis Landsberg erecta x Antwerp-1 population. Frontiers in Plant Science 5: 609

Schneeberger K, Ossowski S, Lanz C, Juul T, Petersen AH, Nielsen KL, Jørgensen JE, Weigel D, Andersen SU (2009) SHOREmap: Simultaneous mapping and mutation identification by deep sequencing. Nature Methods 6: 550-551

Schneider CA, Rasband WS, Eliceiri KW (2012) NIH Image to ImageJ: 25 years of image analysis. Nature Methods 9: 671

Scholz G, Becker R, Pich A, Stephan UW (1992) Nicotianamine - a common constituent of strategies I and II of iron acquisition by plants: A review. Journal of Plant Nutrition 15: $1647-1665$

Schrader EK, Harstad KG, Matouschek A (2009) Targeting proteins for degradation. Nature chemical biology 5: 815-822 
Scott Reid T, Terry KL, Casey PJ, Beese LS (2004) Crystallographic Analysis of CaaX Prenyltransferases Complexed with Substrates Defines Rules of Protein Substrate Selectivity. Journal of Molecular Biology 343: 417-433

Shalem O, Dahan O, Levo M, Martinez MR, Furman I, Segal E, Pilpel Y (2008) Transient transcriptional responses to stress are generated by opposing effects of mRNA production and degradation. Molecular Systems Biology 4: 223-223

Shanmugam V, Lo JC, Wu CL, Wang SL, Lai CC, Connolly EL, Huang JL, Yeh KC (2011) Differential expression and regulation of iron-regulated metal transporters in Arabidopsis halleri and Arabidopsis thaliana - the role in zinc tolerance. New Phytologist 190: 125-137

Shannon P, Markiel A, Ozier O, Baliga NS, Wang JT, Ramage D, Amin N, Schwikowski B, Ideker T (2003) Cytoscape: A Software Environment for Integrated Models of Biomolecular Interaction Networks. Genome Research 13: 2498-2504

Shimada TL, Shimada T, Hara-Nishimura I (2010) A rapid and non-destructive screenable marker, FAST, for identifying transformed seeds of Arabidopsis thaliana: TECHNICAL ADVANCE. Plant Journal 61: 519-528

Silverstone AL, Mak PYA, Martinez EC, Sun T (1997) The New Rga Locus Encodes a Negative Regulator of Gibberellin Response in Arabidopsis Thaliana. Genetics 146: 1087-1099

Sinclair SA, Krämer U (2012) The zinc homeostasis network of land plants. Biochimica et Biophysica Acta - Molecular Cell Research 1823: 1553-1567

Sinclair SA, Sherson SM, Jarvis R, Camakaris J, Cobbett CS (2007) The use of the zincfluorophore, Zinpyr-1, in the study of zinc homeostasis in Arabidopsis roots: Rapid report. New Phytologist 174: 39-45

Sivitz AB, Hermand V, Curie C, Vert G (2012) Arabidopsis bHLH100 and bHLH101 Control Iron Homeostasis via a FIT-Independent Pathway. PLoS ONE 7: e44843

Smaczniak C, Immink RGH, Muiño JM, Blanvillain R, Busscher M, Busscher-Lange J, Dinh QD, Liu S, Westphal AH, Boeren S, Parcy F, Xu L, Carles CC, Angenent GC, Kaufmann K (2012) Characterization of MADS-domain transcription factor complexes in Arabidopsis flower development. Proceedings of the National Academy of Sciences 109: 1560-1565

Smyth Gordon K (2004) Linear Models and Empirical Bayes Methods for Assessing Differential Expression in Microarray Experiments. In Statistical Applications in Genetics and Molecular Biology, Vol 3 
Solovieff N, Cotsapas C, Lee PH, Purcell SM, Smoller JW (2013) Pleiotropy in complex traits: challenges and strategies. Nature Reviews Genetics 14: 483-495

Somerville C (1999) Plant Functional Genomics. Science 285: 380-383

Sommer AL, Lipman CB (1926) EVIDENCE ON THE INDISPENSABLE NATURE OF ZINC AND BORON FOR HIGHER GREEN PLANTS. Plant Physiology 1: 231-249

Song H-R (2012) Interaction between the Late Elongated hypocotyl (LHY) and Early flowering 3 (ELF3) genes in the Arabidopsis circadian clock. Genes \& Genomics 34: 329-337

Song WY, Choi KS, Kim DY, Geisler M, Park J, Vincenzetti V, Schellenberg M, Kim SH, Lim YP, Noh EW, Lee Y, Martinoiaa E (2010) Arabidopsis PCR2 is a zinc exporter involved in both zinc extrusion and long-distance zinc transport. Plant Cell 22: 22372252

Spinner L, Pastuglia M, Belcram K, Pegoraro M, Goussot M, Bouchez D, Schaefer DG (2010) The function of TONNEAU1 in moss reveals ancient mechanisms of division plane specification and cell elongation in land plants. Development 137: 2733-2742

Stes E, Depuydt S, De Keyser A, Matthys C, Audenaert K, Yoneyama K, Werbrouck S, Goormachtig S, Vereecke D (2015) Strigolactones as an auxiliary hormonal defence mechanism against leafy gall syndrome in Arabidopsis thaliana. Journal of Experimental Botany 66: 5123-5134

Sun T-p (2010) Gibberellin-GID1-DELLA: A Pivotal Regulatory Module for Plant Growth and Development. Plant Physiology 154: 567-570

Suzuki N, Yamaguchi Y, Koizumi N, Sano H (2002) Functional characterization of a heavy metal binding protein CdI19 from Arabidopsis. The Plant Journal 32: 165-173

Swarbreck D, Wilks C, Lamesch P, Berardini TZ, Garcia-Hernandez M, Foerster H, Li D, Meyer T, Muller R, Ploetz L, Radenbaugh A, Singh S, Swing V, Tissier C, Zhang P, Huala E (2008) The Arabidopsis Information Resource (TAIR): gene structure and function annotation. Nucleic Acids Research 36: D1009-D1014

Takahashi R, Ishimaru Y, Senoura T, Shimo H, Ishikawa S, Arao T, Nakanishi H, Nishizawa NK (2011) The OsNRAMP1 iron transporter is involved in Cd accumulation in rice. Journal of Experimental Botany 62: 4843-4850

Tan QKG, Irish VF (2006) The Arabidopsis Zinc Finger-Homeodomain Genes Encode Proteins with Unique Biochemical Properties That Are Coordinately Expressed during Floral Development. Plant Physiology 140: 1095-1108 
Tanaka M, Wallace IS, Takano J, Roberts DM, Fujiwara T (2008) NIP6;1 Is a Boric Acid Channel for Preferential Transport of Boron to Growing Shoot Tissues in Arabidopsis. The Plant Cell 20: 2860-2875

Tarantino D, Santo N, Morandini P, Casagrande F, Braun H-P, Heinemeyer J, Vigani G, Soave C, Murgia I (2010) AtFer4 ferritin is a determinant of iron homeostasis in Arabidopsis thaliana heterotrophic cells. Journal of Plant Physiology 167: 1598-1605

Tehseen M, Cairns N, Sherson S, Cobbett CS (2010) Metallochaperone-like genes in Arabidopsis thaliana. Metallomics 2: 556-564

Tester M, Leigh RA (2001) Partitioning of nutrient transport processes in roots. Journal of Experimental Botany 52: 445-457

The Arabidopsis Genome I (2000) Analysis of the genome sequence of the flowering plant Arabidopsis thaliana. Nature 408: 796

Tian G-W, Mohanty A, Chary SN, Li S, Paap B, Drakakaki G, Kopec CD, Li J, Ehrhardt D, Jackson D, Rhee SY, Raikhel NV, Citovsky V (2004) High-Throughput Fluorescent Tagging of Full-Length Arabidopsis Gene Products in Planta. Plant Physiology 135: 25-38

Todd WR, Elvehjem CA, Hart EB (1980) Zinc in the nutrition of the rat. Nutrition Reviews 38: $151-154$

Todesco M, Balasubramanian S, Hu TT, Traw MB, Horton M, Epple P, Kuhns C, Sureshkumar S, Schwartz C, Lanz C, Laitinen RAE, Huang Y, Chory J, Lipka V, Borevitz JO, Dangl JL, Bergelson J, Nordborg M, Weigel D (2010) Natural allelic variation underlying a major fitness tradeoff in Arabidopsis thaliana. Nature 465: $632-$ 636

Tomatsu H, Takano J, Takahashi H, Watanabe-Takahashi A, Shibagaki N, Fujiwara T (2007) An Arabidopsis thaliana high-affinity molybdate transporter required for efficient uptake of molybdate from soil. Proceedings of the National Academy of Sciences of the United States of America 104: 18807-18812

Tsugama D, Liu S, Takano T (2012) A bZIP Protein, VIP1, Is a Regulator of Osmosensory Signaling in Arabidopsis. Plant Physiology 159: 144-155

Uversky VN, Gillespie JR, Fink AL (2000) Why are "natively unfolded" proteins unstructured under physiologic conditions? Proteins: Structure, Function, and Bioinformatics 41: 415-427

van de Mortel JE, Almar Villanueva L, Schat H, Kwekkeboom J, Coughlan S, Moerland PD, Ver Loren van Themaat E, Koornneef M, Aarts MGM (2006) Large Expression 
Differences in Genes for Iron and Zinc Homeostasis, Stress Response, and Lignin Biosynthesis Distinguish Roots of Arabidopsis thaliana and the Related Metal Hyperaccumulator Thlaspi caerulescens. Plant Physiology 142: 1127-1147

van der Lee R, Buljan M, Lang B, Weatheritt RJ, Daughdrill GW, Dunker AK, Fuxreiter M, Gough J, Gsponer J, Jones DT, Kim PM, Kriwacki RW, Oldfield CJ, Pappu RV, Tompa P, Uversky VN, Wright PE, Babu MM (2014) Classification of Intrinsically Disordered Regions and Proteins. Chemical Reviews 114: 6589-6631

Van Roey K, Gibson TJ, Davey NE (2012) Motif switches: decision-making in cell regulation. Current Opinion in Structural Biology 22: 378-385

van Wersch R, Li X, Zhang Y (2016) Mighty Dwarfs: Arabidopsis Autoimmune Mutants and Their Usages in Genetic Dissection of Plant Immunity. Frontiers in Plant Science 7: 1717

Varotto C, Maiwald D, Pesaresi P, Jahns P, Salamini F, Leister D (2002) The metal ion transporter IRT1 is necessary for iron homeostasis and efficient photosynthesis in Arabidopsis thaliana. The Plant Journal 31: 589-599

Vellosillo T, Martínez M, López MA, Vicente J, Cascón T, Dolan L, Hamberg M, Castresana C (2007) Oxylipins Produced by the 9-Lipoxygenase Pathway in Arabidopsis Regulate Lateral Root Development and Defense Responses through a Specific Signaling Cascade. The Plant Cell 19: 831-846

Verma V, Ravindran P, Kumar PP (2016) Plant hormone-mediated regulation of stress responses. BMC Plant Biology 16: 86

Vert G, Briat JF, Curie C (2001) Arabidopsis IRT2 gene encodes a root-periphery iron transporter. Plant Journal 26: 181-189

Vert G, Chory J (2009) A Toggle Switch in Plant Nitrate Uptake. Cell 138: 1064-1066

Vinocur B, Altman A (2005) Recent advances in engineering plant tolerance to abiotic stress: achievements and limitations. Current opinion in biotechnology 16: 123-132

Waldron KJ, Rutherford JC, Ford D, Robinson NJ (2009) Metalloproteins and metal sensing. Nature 460: 823

Wang N, Cui Y, Liu Y, Fan H, Du J, Huang Z, Yuan Y, Wu H, Ling H-Q (2013) Requirement and Functional Redundancy of Ib Subgroup bHLH Proteins for Iron Deficiency Responses and Uptake in Arabidopsis thaliana. Molecular Plant 6: 503-513

Waters BM, Chu HH, DiDonato RJ, Roberts LA, Eisley RB, Lahner B, Salt DE, Walker EL (2006) Mutations in Arabidopsis Yellow Stripe-Like1 and Yellow Stripe-Like3 
reveal their roles in metal ion homeostasis and loading of metal ions in seeds. Plant Physiology 141: 1446-1458

Waters MT, Wang P, Korkaric M, Capper RG, Saunders NJ, Langdale JA (2009) GLK Transcription Factors Coordinate Expression of the Photosynthetic Apparatus in Arabidopsis. The Plant Cell 21: 1109-1128

Weber K, Burow M (2018) Nitrogen - essential macronutrient and signal controlling flowering time. Physiologia Plantarum 162: 251-260

Weigel D (2012) Natural variation in arabidopsis: From molecular genetics to ecological genomics. Plant Physiology 158: 2-22

Weigel D, Mott R (2009) The 1001 Genomes Project for Arabidopsis thaliana. Genome Biology 10: 107-107

Weis-Garcia F, Bandura D, Baranov V, Ornatsky O, Tanner S (2013) The Means: Cytometry and Mass Spectrometry Converge in a Single Cell Deep Profiling Platform. Journal of Biomolecular Techniques : JBT 24: S12-S12

Williams RJP (1984) Zinc: what is its role in biology? Endeavour 8: 65-70

Winter D, Vinegar B, Nahal H, Ammar R, Wilson GV, Provart NJ (2007) An "Electronic Fluorescent Pictograph" Browser for Exploring and Analyzing Large-Scale Biological Data Sets. PLOS ONE 2: e718

Wintz H, Fox T, Wu Y-Y, Feng V, Chen W, Chang H-S, Zhu T, Vulpe C (2003) Expression Profiles of Arabidopsis thaliana in Mineral Deficiencies Reveal Novel Transporters Involved in Metal Homeostasis. Journal of Biological Chemistry 278: 47644-47653

Wintz H, Vulpe C (2002) Plant copper chaperones. Biochemical Society Transactions 30: 732735

Wolters H, Jürgens G (2009) Survival of the flexible: hormonal growth control and adaptation in plant development. 10: 305

Woodroofe CC, Masalha R, Barnes KR, Frederickson CJ, Lippard SJ (2004) MembranePermeable and -Impermeable Sensors of the Zinpyr Family and Their Application to Imaging of Hippocampal Zinc In Vivo. Chemistry \& Biology 11: 1659-1666

Wright PE, Dyson HJ (1999) Intrinsically unstructured proteins: re-assessing the protein structure-function paradigm. Journal of Molecular Biology 293: 321-331

Wu H, Chen C, Du J, Liu H, Cui Y, Zhang Y, He Y, Wang Y, Chu C, Feng Z, Li J, Ling H-Q (2012) Co-Overexpression FIT with AtbHLH38 or AtbHLH39 in ArabidopsisEnhanced Cadmium Tolerance via Increased Cadmium Sequestration in Roots and Improved Iron Homeostasis of Shoots. Plant Physiology 158: 790-800 
Wu Y, Zhao Q, Gao L, Yu X-M, Fang P, Oliver DJ, Xiang C-B (2010) Isolation and characterization of low-sulphur-tolerant mutants of Arabidopsis. Journal of Experimental Botany 61: 3407-3422

Xu F, Huang Y, Li L, Gannon P, Linster E, Huber M, Kapos P, Bienvenut W, Polevoda B, Meinnel T, Hell R, Giglione C, Zhang Y, Wirtz M, Chen S, Li X (2015) Two NTerminal Acetyltransferases Antagonistically Regulate the Stability of a Nod-Like Receptor in Arabidopsis. The Plant Cell 27: 1547-1562

Xu J, Li H-D, Chen L-Q, Wang Y, Liu L-L, He L, Wu W-H (2006) A Protein Kinase, Interacting with Two Calcineurin B-like Proteins, Regulates K+Transporter AKT1 in Arabidopsis. Cell 125: 1347-1360

Yan JY, Li CX, Sun L, Ren JY, Li GX, Ding ZJ, Zheng SJ (2016) A WRKY Transcription Factor Regulates Fe Translocation under Fe Deficiency. Plant Physiology 171: 20172027

Yang J, Benyamin B, McEvoy BP, Gordon S, Henders AK, Nyholt DR, Madden PA, Heath AC, Martin NG, Montgomery GW, Goddard ME, Visscher PM (2010) Common SNPs explain a large proportion of the heritability for human height. Nature genetics 42: 565-569

Yang Z, Tian L, Latoszek-Green M, Brown D, Wu K (2005) Arabidopsis ERF4 is a transcriptional repressor capable of modulating ethylene and abscisic acid responses. Plant Molecular Biology 58: 585-596

Yosef N, Regev A (2011) Impulse control: Temporal dynamics in gene transcription. Cell 144: 886-896

Yruela I (2013) Transition metals in plant photosynthesis. Metallomics 5: 1090-1109

Yu J-H, Hamari Z, Han K-H, Seo J-A, Reyes-Domínguez Y, Scazzocchio C (2004) Doublejoint PCR: a PCR-based molecular tool for gene manipulations in filamentous fungi. Fungal Genetics and Biology 41: 973-981

Yu Q, Tian H, Yue K, Liu J, Zhang B, Li X, Ding Z (2016) A P-Loop NTPase Regulates Quiescent Center Cell Division and Distal Stem Cell Identity through the Regulation of ROS Homeostasis in Arabidopsis Root. PLOS Genetics 12: e1006175

Yuan Y, Wu H, Wang N, Li J, Zhao W, Du J, Wang D, Ling H-Q (2008) FIT interacts with AtbHLH38 and AtbHLH39 in regulating iron uptake gene expression for iron homeostasis in Arabidopsis. 18: 385-397 
Zamioudis C, Hanson J, Pieterse CMJC (2014) $\beta$-Glucosidase BGLU42 is a MYB72dependent key regulator of rhizobacteria-induced systemic resistance and modulates iron deficiency responses in Arabidopsis roots. New Phytologist 204: 368-379

Zamioudis C, Korteland J, Van Pelt JA, van Hamersveld M, Dombrowski N, Bai Y, Hanson J, Van Verk MC, Ling H-Q, Schulze-Lefert P, Pieterse CMJ (2015) Rhizobacterial volatiles and photosynthesis-related signals coordinate MYB72 expression in Arabidopsis roots during onset of induced systemic resistance and irondeficiency responses. The Plant Journal 84: 309-322

Zhang F, Qi B, Wang L, Zhao B, Rode S, Riggan ND, Ecker JR, Qiao H (2016) EIN2dependent regulation of acetylation of histone $\mathrm{H} 3 \mathrm{~K} 14$ and non-canonical histone H3K23 in ethylene signalling. Nature Communications 7: 13018

Zhang F, Wang L, Ko EE, Shao K, Qiao H (2018) Histone Deacetylases SRT1 and SRT2 Interact with ENAP1 to Mediate Ethylene-Induced Transcriptional Repression. The Plant Cell 30: 153-166

Zhang F, Wang L, Qi B, Zhao B, Ko EE, Riggan ND, Chin K, Qiao H (2017) EIN2 mediates direct regulation of histone acetylation in the ethylene response. Proceedings of the National Academy of Sciences 114: 10274-10279

Zhang J, Liu B, Li M, Feng D, Jin H, Wang P, Liu J, Xiong F, Wang J, Wang HB (2015) The bHLH transcription factor bHLH104 interacts with IAA-LEUCINE RESISTANT3 and modulates iron homeostasis in Arabidopsis. Plant Cell 27: 787-805

Zhang X, Huai J, Shang F, Xu G, Tang W, Jing Y, Lin R (2017) A PIF1/PIF3-HY5-BBX23 Transcription Factor Cascade Affects Photomorphogenesis. Plant Physiology 174: $2487-2500$

Zhang Y, Goritschnig S, Dong X, Li X (2003) A Gain-of-Function Mutation in a Plant Disease Resistance Gene Leads to Constitutive Activation of Downstream Signal Transduction Pathways in suppressor of npr1-1, constitutive 1. The Plant Cell 15: 2636-2646

Zhao H, Eide DJ (1997) Zap1p, a metalloregulatory protein involved in zinc-responsive transcriptional regulation in Saccharomyces cerevisiae. Molecular and Cellular Biology 17: $5044-5052$

Zhu J-K (2016) Abiotic Stress Signaling and Responses in Plants. Cell 167: 313-324

Zschiesche W, Barth O, Daniel K, Böhme S, Rausche J, Humbeck KC (2015) The zincbinding nuclear protein HIPP3 acts as an upstream regulator of the salicylate-dependent plant immunity pathway and of flowering time in Arabidopsis thaliana. New Phytologist 207: 1084-1096 
Zumajo-Cardona C, Pabón-Mora N (2016) Evolution of the APETALA2 Gene Lineage in Seed Plants. Molecular Biology and Evolution 33: 1818-1832 



\section{Summary}


$\mathrm{Zn}$ is an essential element for life and the second most abundant transition element in all organisms. $\mathrm{Zn}$ deficiency is one of most widespread micronutrient deficiencies in soils and plants need to cope with it. Plants are able to sense the shortage of $\mathrm{Zn}$ supply and adjust their $\mathrm{Zn}$ homeostasis accordingly. In this thesis, I explored the genetic architecture of the $\mathrm{Zn}$ deficiency response of Arabidopsis thaliana. I initiated functional analyses on some of the $\mathrm{Zn}$ transporter genes long known to be transcriptionally induced by $\mathrm{Zn}$ deficiency, but so far not investigated in much detail. Soon after the exposure to $\mathrm{Zn}$ deficiency the expression of these transporters is first induced in roots and later in shoots. Each $\mathrm{Zn}$ transporter gene is expressed in one, or more, specific root cell layer(s), which location may differ, depending on the root part. These transporters showed partial functional redundancy among them, which makes it difficult, if not impossible, to detect particular $\mathrm{Zn}$ deficiency phenotypes in single $\mathrm{Zn}$ transporter mutants (Chapter 2). Considering that the effect of mutations on $\mathrm{Zn}$ transporter genes is difficult to detect, the $\mathrm{Zn}$ deficiency response can be studied from the perspective of their regulatory components. In the search of these regulators I found that the disruption of the N-ALPHA-TERMINAL ACETYLTRANSFERASE 25 (NAA25) gene, involved in N-terminalacetylation of proteins, causes a strong Fe deficiency sensitivity and a slightly, but consistently, higher expression of several $\mathrm{Zn}$ deficiency responsive genes upon $\mathrm{Zn}$ sufficiency, when compared to wild-type plants. The causes for the unusual expression pattern of these genes still remain unknown. One hypothesis I postulate is that the lack of N-terminal acetylation of the $\mathrm{Zn}$ deficiency regulators bZIP19 and bZIP23, which are possible targets N-terminal acetylation, alters their regulatory activity (Chapter $\mathbf{3})$.

To identify more unconventional genetic players underlying the $\mathrm{Zn}$ deficiency response, that is not the known suspects involved in $\mathrm{Zn}$ homeostasis, such as the transporter genes, the bZIP19/23 transcription factors or the genes involved in Zn chelation, I investigated the natural variation for the Arabidopsis ionome of plants grown under Zn deficiency, in a genome wide association approach (Chapter 4). The loss of function of seven genes, identified in this approach, each causes an increase in ionome profile changes due to $\mathrm{Zn}$ deficiency, some of the element concentrations significantly affected by the loss of function of these genes are those for $\mathrm{Fe}, \mathrm{Mn}$ and $\mathrm{Cu}$. The identified genes are involved in various, diverse, functions such as microtubule organization during cell division, defence against pathogens, control of the circadian clock, Fe storage and phosphorylation of carbohydrates. Thus, their role in $\mathrm{Zn}$ deficiency response can be direct or mediated. The genome wide association study also identified a cluster of five tandemly arrayed genes, encoding for HEAVY METAL- 
ASSOCIATED ISOPRENYLATED PLANT PROTEINS (HIPPs). Functional analyses of these HIPPs suggest they function as negative plant-growth regulators induced by $\mathrm{Zn}$ and Fe deficiency (Chapter 5). Single hipp mutant lines are tolerant to $\mathrm{Zn}$ and especially Fe deficiency, while constitutive overexpression of HIPP10 reduces plant size. Yeast 2-hybrid analysis identified several transcription factors to interact with one or more HIPPs, suggesting these HIPPs to be part of a regulatory network controlling plant growth in response to adverse $\mathrm{Zn}$ and Fe supply.

In conclusion, this thesis contributes to our understanding of the complexity of the Arabidopsis $\mathrm{Zn}$ deficiency response, with analysis of obvious and much less obvious genetic factors contributing to the $\mathrm{Zn}$ deficiency response. While again a small part of the puzzle is completed, several new leads are uncovered in this thesis, which would be of great interest to follow up on. Such investigations are likely to bring us one step further in improving the $\mathrm{Zn}$ content of plant products and the $\mathrm{Zn}$ deficiency tolerance of crops. 



\section{Acknowledgments}

Seven years ago I moved to The Netherlands to pursue a Master's degree, and afterwards I decided to stay doing my $\mathrm{PhD}$. Time flew, and I could not have accomplished this journey without all the wonderful people around me.

I would like to thank my promotor Mark for helping me to learn and understand so much during my PhD. Mark, you always found time to discuss my results or doubts about the experiments. I truly appreciate your guidance, encouragement and patience, along the ups and downs during my PhD. I also would like to thank my co-promotor Maarten for the comments and advice on my research. Maarten, your positive attitude and kindness were very motivating.

The experiments showed in this thesis could not have been done without the help of several people. Thanks Henk, Corrie, Frank, Ana Carolina, Ana A, and Joost v/d H from the Laboratory of Genetics; Richard, Alice, and Tjitske from the Laboratory of Biosciences; Patrick from the Laboratory of Entomology; Eric and Markus from the University of Heidelberg; and David, Paula and John from the University of Nottingham, for your contribution to this thesis in the experiments and data analysis. I also had the opportunity to supervise $\mathrm{PhD}, \mathrm{MSc}$ and $\mathrm{BSc}$ students, who helped me to perform several experiments. Jeroen, María, Aron, Cindy, Aaliya and Jos thanks for the effort and enthusiasm while working with me in this thesis.

The Genetics group has been a great place to work due to all its members. Thanks Ana Carolina, Andrew, Anneloes, Arjan, Bas, Bertha, Charles, Cris, Duur, Diego, Edouard, Erik, Florien, Fons, Gabriella, Hans, Jelle, Jitpanu, Jose, Justin, Klaas, Krithi, Kim, Lennart, Margo, Mathijs, Mark Z, Mina, Mohamed, Pádraic, Pingping, Ramón, René, Roel, Ross, Roxanne, Sijmen, Sabine, Sarah, Tom, Tina, Vanessa and Yanli for making my time in the Genetics group really enjoyable, for all conversation over lunches, coffee breaks, all the fun in our "lab-uitjes", Christmas dinners, and other social events.

Wytske and Corrie, thank you for being there for me every time I needed you during these years. Robert and Tânia, my two post-docs, it was such a pleasure to meet you both. I am grateful for your friendship, guidance, and our chats. In addition, we could always comfort each other about the struggles with our hydroponics experiments. 
During my PhD I met wonderful people and made great friends. Aina, Alex, Alexander, Ana, Ana Paula, André, Amalia, Aranka, Chara, Elise, Eric, Giovanni, Greice, Jianhua, Jose H, Jose M, Paola, and Phoung, thanks for the fun and the time shared during dinners, picnics, trips, tennis training, races, game nights, and so much more.

Bea, Claudio, Lidia, Tânia, and Luchito, thank you for your invaluable friendship, companionship, advice, and laughs. Ineke and Martien, I was very lucky to live in your house. You made me feel at home, not just in a house. I truly enjoyed our dinners and long chats. You were all like family to me in a country where I had none. I will keep you all in my heart.

Finally, I would like to thank my family for being always there for me somehow. Dani, thank you for your help in my experiments and for your support especially during this last stretch of my PhD. I am happy you decided to come with me to Ecuador and I look forward to our years ahead. Maricela, Germán y Edwin gracias por su cariño y compañía en los veranos y navidades en España, me hicieron sentir en casa aun estando tan lejos de ella. A mis padres, Elena y Juan, gracias por el amor, apoyo y confianza incondicional que siempre me han dado. Todo lo que he alcanzado en la vida se lo debo a ustedes. Por eso con todo mi cariño les dedico esta tesis. 


\section{Curriculum Vitae}

Andrea Valeria Ochoa Tufiño was born on 24 August, 1984 in Pallatanga, Ecuador. She spent her childhood in her grandparents farm, where her fascination for plants began. In 2002 she obtained her high school degree at Colegio Nacional Natalia Jarrín in Cayambe and moved to San Rafael to study Biotechnology Engineering. In her graduation research, Valeria studied the genetic variation of Polylepis, a high-altitude native tree. In 2008, Valeria graduated at Universidad de las Fuerzas Armadas-ESPE, and started to work as a lecturer and research assistant. In 2011, after obtaining a scholarship to study abroad, she decide to pursue a Master's degree in Plant Biotechnology at Wageningen University. Her master's research was conducted in the laboratory of Genetics, and it was about the characterization of a gene involved in heavy metals tolerance and accumulation in Noccaea caerulescens. In 2013, she obtained a grant for her $\mathrm{PhD}$ research and started it in Wageningen University, at the Laboratory of Genetics under the supervision of Prof. Dr Mark Aarts and Prof. Dr Maarteen Koorneef, which has led to this thesis. 


\section{Publications}

Ochoa, V., Jadán, M., Jiménez, P., Segovia, C. and Proaño, K. (2008). Population genetics of Polylepis pauta and Polylepis incana in Papallacta with ISSRs. Ciencia y Tecnología 1:71-79.

Ochoa AV, Almira M, van Duynhoven A, Aarts MGM. Uncovering Zn transporters induced by $\mathrm{Zn}$ deficiency. In preparation

Ochoa AV, Campos AC, van den Heuvel J, Assunção AGL, Linster E, Wirtz M, Aarts MGM. Disruption of N-terminal-acetylation disturbs mineral homeostasis in Arabidopsis thaliana. In preparation

Ochoa AV, Campos AC, van den Heuvel J, Wolfkamp J, Danku J, Salt DE, Aarts MGM. Exploring Arabidopsis thaliana natural genetic variation to identify genes underlying the $\mathrm{Zn}$ deficiency response. In preparation

Ochoa AV, Aaliya K, Meeussen J, Aarts MGM. A conserver cluster of tandemly arrayed HIPP genes affects tolerance to $\mathrm{Fe}$ and $\mathrm{Zn}$ deficiency in Arabidopsis thaliana. In preparation 


\title{
Education Statement of the Graduate School \\ Experimental Plant Sciences
}

\author{
Issued to: Andrea Valeria Ochoa Tufiño \\ Date: $\quad 05$ October 2018 \\ Group: Laboratory of Genetics \\ University: Wageningen University \& Research
}

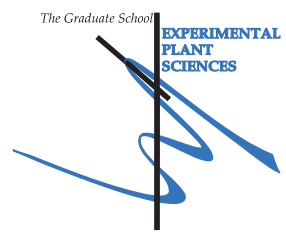

\begin{tabular}{|l|l|}
\hline 1) Start-up phase \\
First presentation of your project \\
Analysis of the regulatory network of Zn in Arabidopsis thaliana \\
Writing or rewriting a project proposal \\
Analysis of the regulatory network of Zn in Arabidopsis thaliana \\
Writing a review or book chapter \\
MSc courses \\
Laboratory use of isotopes
\end{tabular}

Subtotal Start-up Phase

6.5 credits *

\section{2) Scientific Exposure}

- EPS PhD student days

EPS PhD student day 'Get2Gether', Soest, The Netherlands

date

EPS PhD student day 'Get2Gether', Soest, The Netherlands

- EPS theme symposia

EPS Theme 3 Symposium 'Metabolism and Adaptation', Wageningen, The Netherlands

EPS Theme 4 Symposium 'Genome Biology', Wageningen, The Netherlands

EPS Theme 3 Symposium 'Metabolism and Adaptation', Wageningen, The Netherlands

EPS Theme 4 Symposium 'Genome Biology', Amsterdam, The Netherlands

EPS Theme 3 Symposium 'Metabolism and Adaptation', Amsterdam, The Netherlands

EPS Theme 3 Symposium 'Metabolism and Adaptation', Wageningen, The Netherlands

29-30 Jan 2015

28-29 Jan 2016

National meetings (e.g. Lunteren days) and other National Platforms

Annual meeting 'Experimental Plant Sciences', Lunteren, The Netherlands

Annual meeting 'Experimental Plant Sciences', Lunteren, The Netherlands

Annual meeting 'Experimental Plant Sciences', Lunteren, The Netherlands

Annual meeting 'Experimental Plant Sciences', Lunteren, The Netherlands

- Seminars (series), workshops and symposia

Startsymposium: Plant Developmental Biology, Wageningen, The Netherlands

Seminar Series: Genetic Seminar Series (weekly)

Seminar: Ionomics - Genomis of mineral nutrients and trace elements in plant - David Salt, Amsterdam, The Netherlands

Seminar: On Bees, Pollination and Food Security - Koos Biesmeijer, Wageningen, The Netherlands

Seminar: Genetics and epigenetics: a complex relationship - Ortrun Mittelsten Scheid, Wageningen, The

Netherlands

Seminar: The evolutionary significance of gene and genome duplications - Yves van de Peer,

Wageningen, The Netherlands

Seminar: Identification of a new sensory neuron membrane gene and why phylogenomics is important -

Alexie Papanicolaou, Wageningen, The Netherlands

Seminar: Dynamics of Adaptation and Genome Evolution in a Long-Term Experiment - John Hanna,

Wageningen, The Netherlands

Seminar plus

- International symposia and congresses

Agronomic, Molecular Genetics and Human Nutrition Approaches for Improving the Nutritional Quality and Safety of Food Crops Conference, Antalya, Turkey

LOCOMET international network (GDRI CNRS) meeting, Lille, France

26th International Conference on Arabidopsis Research (ICAR), Paris, France

4th International Znc Symposium: Improving Crop Production and Human Health, Sao Paulo, Brazil

27th International Conference on Arabidopsis Research (ICAR), Gyeongju, South Korea

LOCOMET international network (GDRI CNRS) meeting, Lille, France

11 Mar 2014

03 Dec 2014

10 Feb 2015

15 Dec 2015

23 Feb 2016

14 Mar 2017

14-15 Apr 2014

13-14 Apr 2015

11-12 Apr 2016

10-11 Apr 2017

14 Oct 2013

2013-2017

23 Oct 2014

18 Dec 2014

19 Nov 2014

03 Feb 2015

22 Jun 2017

31 Aug 2017

XVIII International Plant Nutrition Colloquium (IPNC), Copenhagen, Denmark

17-19 Mar 2014

02-04 Jul 2014

05-09 Jul 2015

15-17 Sep 2015

29 Jun-03 Jul 2016

07-08 Jul 2016

21-24 Aug 2017 


\section{- Presentations}

Poster: Agronomic, Molecular Genetics and Human Nutrition Approaches for Improving the Nutritional Quality and Safety of Food Crops Conference, Antalya, Turkey

Poster: Annual meeting 'Experimental Plant Sciences', Lunteren, The Netherlands

Poster: 26th International Conference on Arabidopsis Research (ICAR), Paris, France

Poster: 27th International Conference on Arabidopsis Research (ICAR), Gyeongju, South Korea

Talk: 4th International Zinc Symposium: Improving Crop Production and Human Health, Sao Paulo, Brazil

Talk: LOCOMET international network (GDRI CNRS) meeting, Lille, France

Talk: EPS Theme 3 Symposium 'Metabolism and Adaptation', Wageningen, The Netherlands

Talk: Annual meeting 'Experimental Plant Sciences', Lunteren, The Netherlands

Talk: XVIII International Plant Nutrition Colloquium (IPNC), Copenhagen, Denmark

7-19 Mar 2014

13-14 Apr 2015

05-09 Jul 2015

29 Jun-03 Jul 2016

15-17 Sep 2015

07-08 Jul 2016

14 Mar 2017

10-11 Apr 2017

21-24 Aug 2017

IAB interview

- Excursions

\section{Subtotal Scientific Exposure}

25.1 credits

\section{3) In-Depth Studies}

- EPS courses or other PhD courses

Transcription Factors and Transcriptional Regulation, Wageningen, The Netherlands

School of Plant Metallomics, Copenhagen, Denmark

Introduction to R for Statistical Analysis, Wageningen, The Netherlands

Genome Assembly, Wageningen, The Netherlands

Basic Statistics, Wageningen, The Netherlands

Linear Models, Wageningen, The Netherlands

Generalized Linear Models, Wageningen, The Netherlands

Mixed Linear Models, Wageningen, The Netherlands

Image Analysis for Plant Phenotyping, Wageningen, The Netherlands

Journal club

Group of Plant Genetics

Individual research training

\section{date}

17-19 Dec 2013

27-31 Jan 2014

23-24 Oct 2014

28-29 Apr 2015

20-27 May 2015

08-10 Jun 2015

15-16 Jun 2015

22-23 Jun 2015

12-15 Jul 2016

2013-2016

Subtotal In-Depth Studies

9.5 credits *

\section{4) Personal development}

- Skill training courses

PhD Competence Assessment, Wageningen, The Netherlands

Techniques for Writing and Presenting a Scientific Paper, Wageningen, The Netherlands

Scientific Writing, Wageningen, The Netherlands

Adobe Indesign Essential Training, Wageningen, The Netherlands

\section{$\underline{\text { date }}$}

04-19 Nov 2014

14-17 Oct 2014

Nov 2016 - Jan 2017

Organisation of PhD students day, course or conference

09-10 Oct 2017

- Membership of Board, Committee or PhD council

$$
\text { Subtotal Personal Development }
$$

3.7 credits *

\section{TOTAL NUMBER OF CREDIT POINTS}

44.8 credits

Herewith the Graduate School declares that the $\mathrm{PhD}$ candidate has complied with the educational requirements

set by the Educational Committee of EPS which comprises of a minimum total of 30 ECTS credits

${ }^{*}$ A credit represents a normative study load of 28 hours of study. 
The research described in this thesis was carried out at Laboratory of Genetics at Wageningen University, Wageningen, The Netherlands, and was financially supported by the Ecuadorian government through SENESCYT (Secretaría de Educación Superior, Ciencia, Tecnología e Innovación) and Universidad de las Fuerzas Armadas-ESPE.

\section{Colophon}

Cover design and thesis layout by: Andrea Valeria Ochoa Tufiño

Printed by: ProefschriftMaken 